UNIVERSITÉ DU QUÉBEC À CHICOUTIMI

en association avec

UNIVERSITÉ DU QUÉBEC À MONTRÉAL

LE MATÉRIEL BLOC AU CCEUR DE L'ACTIVITÉ COGNITIVE ET SOCIALE DE L'ENFANT À LA MATERNELLE

\author{
THÈSE \\ PRÉSENTÉE \\ COMME EXIGENCE PARTIELLE \\ DU DOCTORAT EN ÉDUCATION
}

\author{
PAR \\ MANON DOUCET
}




\section{Bibliothèque}

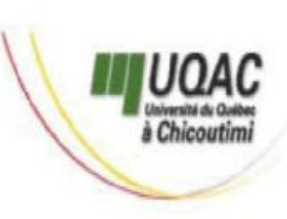

\section{Mise en garde/Advice}

Afin de rendre accessible au plus Motivated by a desire to make the grand nombre le résultat des results of its graduate students' travaux de recherche menés par ses research accessible to all, and in étudiants gradués et dans l'esprit des accordance with the rules règles qui régissent le dépôt et la governing the acceptation and diffusion des mémoires et thèses diffusion of dissertations and produits dans cette Institution, theses in this Institution, the I'Université du Québec à Université du Québec à Chicoutimi (UQAC) est fière de Chicoutimi (UQAC) is proud to rendre accessible une version make a complete version of this complète et gratuite de cette œuvre. work available at no cost to the reader.

L'auteur conserve néanmoins la The author retains ownership of the propriété du droit d'auteur qui copyright of this dissertation or protège ce mémoire ou cette thèse. thesis. Neither the dissertation or Ni le mémoire ou la thèse ni des thesis, nor substantial extracts from extraits substantiels de ceux-ci ne it, may be printed or otherwise peuvent être imprimés ou autrement reproduced without the author's reproduits sans son autorisation. permission. 
À Steen, décédé en 1996, dont le regard positif sur ma démarche continue de m'habiter et de me soutenir.

À Louise, décédée en 1996, de qui j'ai appris le courage, le courage d'aller toujours jusqu'au bout de tout, au bout de nos intuitions, au bout de nos convictions. Le courage de choisir la conscience de sa vie.

À Jessica et Claudel, mes deux filles, qu'elles apprennent très tôt à «Accomplir leur Légende Personnelle, qu'on a toujours la possibilité de faire ce que l'on rêve... Et qu'aucun coeur n'a jamais souffert alors qu'il était à la poursuite de ses rêves.»

Paulo Coelfo 


\section{REMERCIEMENTS}

La démarche de réalisation d'une thèse est le fruit de précieuses et d'étonnantes complicités intellectuelles, affectives et sociales.

Mes premiers remerciements s'adressent aux membres de mon comité de direction. À Madame Jacqueline Thériault, directrice, qui m'a encouragée à entreprendre des études de troisième cycle. Depuis le début de cette démarche, elle a accompagné, confronté et respecté mes choix, mes détours, mes questionnements et mes remises en question épistémologiques, théoriques, méthodologiques et aussi personnelles, puisqu'une telle démarche implique un recadrage de diverses chimies quotidiennes qui constituent notre cheminement de vie. Elle a aiguillonné mes moments «d'eurêka» et remis en perspective mes moments de zones plus sombres où le doute, le manque de confiance et le découragement canalisaient mes sources d'énergie positive.

Son écoute, ses conseils, son ouverture, sa rigueur intellectuelle, sa disponibilité et sa générosité m'ont permis de me réajuster et de me repositionner et m'ont appris à aller toujours un peu plus loin tout au long de ma démarche.

À Monsieur Steen Esbensen, codirecteur, qui m'a accompagnée, soutenue, confrontée, respectée et encouragée à chacune des étapes de la réalisation de cette démarche. Malheureusement, son décès en 1996, m’a empêchée de profiter de son regard critique et positif, de sa rigueur intellectuelle et de sa présence pour les derniers moments de la rédaction.

À Madame Marta Anadón, Présidente de jury de thèse qui, à différents moments cruciaux de cette démarche, m'a constamment apporté son soutien et son encouragement. Ses confrontations sur les plans épistémologique, théorique et méthodologique m'ont 
grandement aidée à me construire comme chercheure et continuent d'être le miroir de considérations que je lui porte.

À Madame Catherine Garnier, Messieurs Claude Germain, Jan Palkiewicz et Renald Legendre qui ont assumé la responsabilité des séminaires d'orientation et de synthèse ainsi que le cours Recherche en éducation. Ces chercheurs de qualité m'ont laissé des empreintes significatives par leurs commentaires rigoureux, positifs et constructifs et par leur accompagnement à mes premiers pas dans le monde de la recherche. Ils m'ont confrontée et confirmée dans mes choix de chercheure et de professionnelle.

À Madame Catherine Garnier et Monsieur Gilles Brougère qui m'ont respectivement accueillie du CIRADE à l'Université du Québec à Montréal et du département des Sciences du Jeu à l'Université de Paris-Nord dans le cadre de mes stages de recherche. Ils m'ont permis d'appréhender et d'apprivoiser différentes visions méthodologiques de la recherche et différents objets de recherche.

Au groupe des « 4 », Lucie Godard, Mireille Provencher et Kathleen Thériault avec qui j'ai partagé diverses réflexions constructivistes.

À Sylvain Tremblay, mon compagnon de vie, dont la présence quotidienne tout au long de cette expérience de recherche est d'une valeur inestimable. Sa patience, son acceptation des exigences et des contraintes rattachées à ce travail et surtout ses qualités personnelles, son respect pour moi et son soutien constant m'ont aidée à mener jusqu'au bout cette démarche.

À Jessica et Claudel, mes deux filles, qui ont respecté et accepté mes nombreuses absences et qui ont pris le risque de me donner du temps sans douter de la façon dont il leur sera rendu. Ma profonde gratitude à ces trois amours.

À Ghislain, Édith, Katy et Steeve, dont leur support, leur encouragement, leur présence auprès de mes filles et surtout cet héritage de « liberté d'être et de vie » qu'ils m'ont légué, un merci très spécial pour ce travail d'arrière-scène qui m'a permis d'être à l'avantscène. 
À Catherine, France, Rolande, Christiane, Céline, Johanne et Nathalie, mes amies, qui ont écouté et confronté mes réflexions, mes prises de conscience et mes remises en question implicites et sous-jacentes à cette démarche.

À Johanne Beaumont, Esther Cloutier et Françoise Tremblay pour l'aide apportée aux mil et une versions qu'a nécessité la transcription de ce manuscrit.

À Maxime qui a assuré le support technique à la cueillette des données et l'incrustation du temps sur les bandes magnétoscopiques.

À Monsieur Nive Voisine qui m'a encouragée, soutenue et accompagnée dans les derniers moments de la rédaction.

À mes collègues, à mes étudiants et à tous ceux qui m'ont encouragée dans différents moments de ma démarche doctorale.

Je remercie le Conseil de Recherche en Sciences Humaines (CRSH), les fonds FCAR, PAIR et Jean-Marc Dénommé ainsi que le ministère de l'Éducation qui m'ont tour à tour aidée financièrement pour la réalisation et les différentes communications de cette recherche.

UN MERCI TOUT SPÉCIAL à Madame Michèle Bouchard, enseignante, qui m'a ouvert les portes de sa classe maternelle et aux 19 merveilleux enfants de cette classe qui ont accepté de me laisser entrer, quelques instants, dans leur monde imaginaire de jeu. 


\section{TABLE DES MATIÈRES}

REMERCIEMENTS

TABLE DES MATIÈRES

LISTE DES FIGURES

xiii

LISTE DES TABLEAUX

xiiv

RÉSUMÉ

$\mathrm{xv}$

INTRODUCTION

\section{CHAPITRE I}

D'UN QUUESTIONNEMENT MATÉRIEL ET ENVIRONNEMENTAL SUR LES BLOCS À UN QUESTIONNEMENT STRUCTURAL ET INTERACTIONNEL DE L'ACTIVITE DE L'ENFANT

1.1 Vers une compréhension du matériel bloc et de l'activité qu'il suscite .................. $\quad 7$

1.1.1 Les blocs vus comme objet industriel ..............................................................

1.1.2 Les blocs vus comme objet de jeu ................................................................ 12

1.1.3 Les blocs vus comme objet éducatif et pédagogique ....................................... 18

1.1.4 Les blocs vus comme objet de recherche ..................................................... 23

- La recherche sur les blocs selon une perspective psychologique ....... 24

- L'approche psychodynamique ...................................................................... 24

- L'approche développementale ................................................................ 26

- L'approche pédagogique …........................................................................... 31

- La recherche sur les blocs selon une perspective sociale .................. $\quad 33$

1.2 L'énoncé du problème et les questions de recherche …............................................... 37

1.3 Récapitulation - Synthèse ……..................................................................................... 41 
CHAPITRE II

D'UN QUESTIONNEMENT INTUITIF DE L'ACTIVITÉ DE L'ENFANT AVEC LE MATÉRIEL BLOC À UN RECADRAGE ÉPISTÉMOLOGIQUE ET CONCEPTUEL DE L'OBJET DE RECHERCHE

2.1 Vers une compréhension du jeu et du jeu symbolique

2.1.1 Piaget, une conception du développement de l'intelligence et des connaissances où l'aspect individuel est central

2.1.2 Piaget, une conception du jeu et du jeu symbolique à travers le filtre psycho-individuel où l'aspect cognitif est central

- La place des jeux de construction dans la classification de Piaget .......

2.1.3 Vygotsky, une conception du développement de l'intelligence et des connaissances où l'aspect social est central

2.1.4 Vygotsky, une conception du jeu et du jeu symbolique à travers le filtre psychosocial où l'aspect cognitif est central

2.1.5 Vers un renouveau de l'intérêt pour le jeu symbolique

2.2 Vers une compréhension des interactions sociales; du constructivisme au socio-constructivisme: Piaget, Vygotsky, Doise, Mugny et Perret-Clermont...

2.2.1 Piaget, le rôle des interactions sociales dans sa conception du développement de l'intelligence

2.2.2 Vygotsky, le rôle des interactions sociales dans sa conception du développement de l'intelligence

2.2.3 Doise, Mugny, Perret-Clermont, le rôle des interactions sociales dans la conception du développement de l'intelligence

2.2.4 La recherche selon une perspective psychosociale

2.2.5 La recherche sur les interactions sociales

2.2.6 La recherche sur les interactions sociales et le jeu symbolique social .

2.2.7 Vers la formulation des questions de recherche relatives à l'activité de l'enfant avec le matériel bloc

CHAPITRE III

D'UNE PROBLÉMATIQUE DE RECHERCHE SUR L'ACTIVITÉ DE L'ENFANT AVEC LE MATÉRIEL BLOC À SON OPÉRATIONNALISATION: L'APPROCHE OBSERVATIONNELLE

3.1 L'observation comme démarche privilégiée ……............................................................... 86

3.2 Les fondements de l'observation .................................................................................... 87

3.3 La planification de la cueillette des données ....................................................................... 93

3.3.1 La cueillette des données: l'observation systématique ………………............ 93

3.3.2 La cueillette des données: l'observation expérientielle ................................... 96

3.3.3 La décision de l'échantillonnage .......................................................................... 97 
3.3.4 L'instrument d'enregistrement des données ........................................................ 98

3.3.5 La mise en situation pour chaque séance d'observation ................................. 99

3.4 La planification de l'analyse qualitative des données ....................................................... 100

3.4.1 Les stratégies globales de mise en forme des données ................................... 100

CHAPITRE IV

D'UNE MISE EN FORME DES DONNÉES AUX PREMIĖRES PISTES DE COMPRÉHENSION DE L'ACTIVITÉ DE L'ENFANT SUSCITÉE PAR LE MATÉRIEL BLOC

4.1 Première question de recherche: Stades d'évolution de l'activité de construction

4.1.1 Les modalités spécifiques d'analyse des données de la première question

Thème 1: Les données relatives au stade d'évolution de l'activité de construction de recherche

- Observation des différentes productions

- Observation des composantes privilégiées: symétrie et équilibre

- Observation de la communication verbale

Thème 2: Les données relatives aux produits de l'activité

- Première tâche: création d'un contexte

- Deuxième tâche: transposition des idées/négociations avec la réalité

- Troisième tâche: processus de distanciation

Thème 3: Les données relatives au temps consacré à l'activité de construction

- Fonctionnement de la classe maternelle

- Observation de la répartition du temps consacré à l'activité de construction et l'activité de jeu symbolique

- Modalités d'analyse

Thème 4: Les données relatives aux diverses formes de participation sociale

4.2 Deuxième question de recherche: les composantes structurales de l'activité de jeu symbolique à partir du matériel bloc

4.2.1 Les modalités générales d'analyse

- Les unités d'analyse

- Première composante structurale: la décontextualisation et la substitution d'objets

- Deuxième composante structurale: le jeu social ritualisé

- Troisième composante structurale: l'adoption de rôles 
- Quatrième composante structurale: le jeu fantaisiste thématique/le jeu sociodramatique

- Cinquième composante structurale: la structuration du jeu

4.2.2 Les modalités spécifiques d'analyse des données de la deuxième question

- Analyse de la première composante structurale: la substitution d'objets

- Analyse de la deuxième composante structurale: le jeu social ritualisé

- Analyse de la troisième composante structurale: l'adoption de rôles

- Analyse de la quatrième composante structurale: le jeu fantaisiste thématique/le jeu sociodramatique

- Analyse de la cinquième composante structurale: la structuration du jeu

4.2.3 La mise en forme des données

- L'organisation solitaire

- Les substitutions d'objets

- L'adoption de rôles

- L'organisation dyadique

- La simultanéité épisodique de courte durée de l'activité de construction dans l'activité de jeu symbolique

- La simultanéité épisodique de courte durée de l'activité de jeu symbolique dans l'activité de construction

- La simultanéité épisodique de longue durée de l'activité de jeu symbolique dans l'activité de construction

- Les substitutions d'objets et l'activité de jeu symbolique subordonnée à l'activité de construction

- Les substitutions d'objets et l'activité de jeu symbolique simultanée à l'activité de construction

- L'organisation dyadique et l'adoption de rôles

- L'adoption de rôles et l'activité de construction

- L'adoption de rôles et l'activité de jeu symbolique subordonnée à l'activité de construction

- L'adoption de rôles et l'activité symbolique simultanée à l'activité de construction

- L'organisation à quatre

- Les substitutions d'objets

- L'adoption de rôles 
4.3 Troisième question de recherche: Les interactions sociales ....................................... 181

4.3.1 Les négociations interpersonnelles dans l'activité de construction et dans l'activité de jeu symbolique

- Quelques éléments théoriques aux négociations interpersonnelles .... 185

4.3.2 La mise en forme des données ............................................................................... 188

- Les données relatives aux interactions sociales .............................................. 193

- Première phase: le positionnement initial ................................................. 193

- Deuxième phase: l'élaboration de l'activité de construction ......... 199

Les éléments de l'objet de négociation de la deuxième phase

Séquence de désaccord intra-dyade ................................................ 203

Événement de désaccord inter-dyade ............................................. 205

- Troisième phase: l'élaboration de l'activité de jeu symbolique

Les éléments de l'objet de négociation de la troisième phase

Séquence de désaccord intra-dyade 222

Événement de désaccord inter-dyade

\section{CHAPITRE V}

D'UN LIEN THÉORIQUE DE L'ANALYSE DES DONNÉES À L'ÉMERGENCE D'UNE COMPREHENSION ET À L'OUVERTURE DE NOUVEAUX REGARDS POUR LA RECHERCHE ET L'INTERVENTION PÉDAGOGIQUE ..

5.1 Résultats de l'analyse des données relatifs à la première question de recherche

5.1.1 Synthèse des résultats relatifs aux stades d'évolution de l'activité de recherche

5.1.2 Synthèse des résultats relatifs aux produits de l'activité

5.1.3 Synthèse des résultats relatifs au temps consacré à l'activité de construction

5.1.4 Synthèse des résultats relatifs aux diverses formes de participation sociale

5.2 Résultats de l'analyse des données relatifs à la deuxième question de recherche

5.2.1 Synthèse des résultats relatifs aux composantes structurales dans l'organisation solitaire

- Les substituts d'objets 254

- L'adoption de rôles 
5.2.2. Synthèse des résultats relatifs aux composantes structurales dans l'organisation dyadique

- Les substitutions d'objets dans l'activité de jeu symbolique subordonnée à l'activité de construction

- Les substitutions d'objets dans l'activité de jeu symbolique simultanée à l'activité de construction

- L'adoption de rôles dans l'activité de construction

- L'adoption de rôles dans l'activité de jeu symbolique subordonnée à l'activité de construction

- L'adoption de rôles dans l'activité de jeu symbolique simultanée à l'activité de construction

5.2.3 Synthèse des résultats relatifs aux composantes structurales dans l'organisation à quatre

- Les substitutions d'objets

- L'adoption de rôles

5.3 Résultats de l'analyse des données relatifs à la troisième question de recherche

5.3.1 Synthèse des résultats relatifs aux interactions sociales dans la première phase: le positionnement initial

5.3.2 Synthèse des résultats relatifs aux interactions sociales dans la deuxième phase: l'élaboration de l'activité de construction

5.3.3 Synthèse des résultats relatifs aux interactions sociales dans la troisième phase: l'élaboration de l'activité de jeu symbolique

5.4 Émergence d'une compréhension de la dynamique de l'activité de l'enfant avec le matériel bloc

\section{APPENDICE A}

A.1 Blocs de construction en bois vernis de Caroline Pratt

A.2 Identification des différents stades ou étapes d'évolution dans l'activité de construction avec le matériel bloc selon Harriet Johnson (1974), Diane Dodge (1979), Mary Ann Santa (1980), Maria Apelman (1984), et Stuart Reifel (1984)

A.3 Ensemble de projets globaux et secondaires réalisés dans les neuf séances d'observation 
A.4 Identification de l'ensemble des rôles nommés, initiés et joués dans l'activité de jeu symbolique subordonnée à l'activité de construction, selon la classification de Garvey (1977) 


\section{LISTE DES FIGURES}

Figure

2.1 Synthèse relative à l'interrelation des trois questions de recherche de l'activité de l'enfant suscitée par le matériel bloc

3.1 Vision intégrative des trois dimensions du savoir

3.2 Vision intégrative des trois dimensions du savoir spécifique à notre étude 


\section{LISTE DES TABLEAUX}

Tableau

Page

1.1 Récapitulation - Synthèse ………….................................................................................... 41

4.1 Ensemble des éléments indicateurs retenus relatifs aux stades de l'évolution de l'activité de construction

4.2 Les différentes catégories regroupant l'ensemble des productions élaborées par les enfants

4.3 Quelques exemples d'énoncés verbaux de projets globaux et de projets secondaires et complémentaires portant sur des structures à la dimension de la taille des enfants

4.4 La moyenne du temps consacré à l'activité de construction et l'activité de jeu symbolique

4.5 Les différents types de regroupements observés dans l'aire des blocs selon les formes de participation sociale pour chaque séance d'observation

4.6 Identification des éléments d'analyse retenus pour chacune des composantes structurales

4.7 Structure d'organisation de l'activité de construction et de jeu symbolique selon les différentes formes de participation sociale et le temps accordé pour chaque type d'activités

4.8 Les différentes substitutions d'objets des séances dyadiques de l'activité de jeu symbolique subordonnée à l'activité de construction

4.9 La répartition des substitutions symboliques d'objets dans les dyades où l'activité de jeu symbolique est simultanée à l'activité de construction

4.10 Les différentes substitutions d'objets dans l'organisation à quatre

4.11 Découpage en phases, épisodes, séquences et événements de chacune des séances de jeu 


\section{RÉSUMÉ}

L'étude de l'activité suscitée par le matériel bloc chez l'enfant de cinq-six ans qui fréquente la classe maternelle constitue la problématique de cette recherche qui s'inscrit dans une démarche de compréhension d'une dynamique particulière de jeu.

Cette problématique de recherche découle de nos préoccupations relatives à la nature et à la structure des activités de l'enfant avec le matériel bloc qui s'articule autour de deux questionnements: un questionnement matériel et environnemental et un questionnement structural et interactionnel.

L'activité de l'enfant avec le matériel bloc constitue le point central de cette étude descriptive et veut mettre en évidence les potentialités du matériel, les composantes et les produits de cette activité afin de dégager des paramètres spécifiques quant à la nature et la structure de cette activité.

Ces deux pôles de questionnement ont orienté les premiers jalons de nos questions générales et spécifiques de recherche afin d'identifier les différentes activités suscitées par le matériel bloc dans le contexte de la classe maternelle et la contribution potentielle de ce matériel et de ces activités au développement cognitif et social de l'enfant. Quelle est la nature des activités que le matériel bloc suscite chez l'enfant? Est-ce que ce matériel et les activités qu'il suscite contribuent au développement cognitif et social de l'enfant? À quel stade ou à quelle étape d'évolution de l'activité de construction se situent les enfants? Quelles sont les composantes structurales de l'activité de jeu symbolique à partir du matériel bloc? Quels types d'interactions sociales émergent de l'activité de l'enfant avec le matériel bloc?

Autant de questions qu'il nous importait d'élucider pour comprendre la dynamique de l'activité de l'enfant avec le matériel bloc.

À cette fin, la démarche de recherche privilégiée nous a amenée à retracer l'évolution du matériel bloc comme objet industriel, comme objet de jeu, comme objet éducatif et pédagogique et comme objet de recherche afin d'envisager le matériel bloc dans sa complexité en interrogeant la présence, la pertinence, la place, l'accessibilité, la connaissance et l'exploitation de ce matériel dans les classes maternelles.

Pour trouver des pistes de réponses aux différents questionnements, nous avons réalisé un recadrage épistémologique et conceptuel afin de situer notre objet d'étude dans une approche constructiviste et socio-constructiviste où les pensées de Piaget (1964), Vygotsky (1978), Doise et Mugny (1981) et Perret-Clermont (1979) ont constitué la trame de fond pour examiner le développement de la connaissance, de l'enfant, du jeu en général et du jeu symbolique en particulier et finalement, des interactions sociales. Nous avons tenté d'élaborer bien humblement une vision intégrante de ces différentes approches pour comprendre la dynamique de l'activité de l'enfant avec le matériel bloc.

L'opérationnalisation de la problématique de l'étude nous a conduite au choix d'une approche observationnelle dans une démarche qualitative de recherche. Ce choix méthodologique a nécessité une réflexion épistémologique sur les modes de production de 
connaissance, où l'observateur-chercheur construit l'objet qu'il observe, teinté de ses croyances et de ses valeurs et du filtre théorique privilégié en tenant compte des éléments théoriques et émergents de la situation observée. De plus, ce choix méthodologique a exigé différents défis quant à la construction de la démarche et des cadres référentiels de mise en forme et d'analyse tant sur le plan de la cueillette des données que de leurs analyses puisant ses principes de base à la fois dans des techniques d'observation systématique et expérientielle. Les méthodes d'analyse de contenu de L'Écuyer (1990), combinées à la méthode d'analyse de Huberman et Miles (1991), ont composé la trame de mise au point et d'analyse des données issues de nos observations.

Par la suite, ces différentes lectures analytiques des données ont tracé les bases d'interprétation et de compréhension de ces données liées à chacune des trois questions spécifiques de recherche. Ces diverses lectures ont permis de positionner théoriquement les résultats d'analyse afin de dégager les éléments complémentaires et émergents qui s'inscrivent dans une contribution potentielle au développement cognitif et social de l'enfant.

Finalement, l'ensemble de ces données participe à l'élargissement du champ de connaissances quant aux stades d'évolution de l'activité de construction, quant aux différentes composantes et aux différents produits des activités de construction et de jeu symbolique et quant aux interactions sociales qui influencent, déterminent et structurent à la fois le fruit et la source de ces activités.

Ces nouvelles connaissances émergeant du filtre de l'observation et de l'interprétation constructiviste et socio-constructiviste relancent de nouveaux regards théoriques, méthodologiques et pédagogiques sur l'activité de l'enfant avec le matériel bloc afin de comprendre un peu plus et un peu mieux toute la richesse et la complexité de la dynamique de cette activité. 


\section{INTRODUCTION}

La classe maternelle est le point de départ de la grande aventure dans le monde de l'école pour l'enfant de cinq ans. De l'extérieur, tous savent qu'à la maternelle, c'est différent! L'aménagement de cette classe ressemble peu à ce que l'on retrouve dans les classes du primaire. Le matériel, dit éducatif, proposé aux enfants est souvent varié, abondant et présenté de diverses façons. Les activités sont nombreuses et semblent différentes. Le fonctionnement de la classe et même l'intervention de l'enseignante paraissent bien particuliers. De plus, ce que l'on entend dire le plus souvent autant de la part des adultes que des autres enfants de l'école, c'est à la maternelle c'est pas pareil, à la maternelle on joue. Mais ce "on joue" peut prendre des allures bien particulières et des sens bien relatifs, dépendant du regard que l'on pose sur cette activité ludique des enfants et du développement de celui-ci.

À chaque période importante de son développement, le on joue de l'enfant peut se qualifier de façon très spécifique. Pour le jeune bébé, l'exploration des objets par la manipulation et l'exploration par les autres sens constitue son activité la plus importante pour connaître ces objets et pour jouer avec eux. Ces jeux sont alors qualifiés de sensori-moteurs.

En continuant ses progrès de connaissances du monde, l'enfant, munit d'une fonction supplémentaire, la représentation, apporte des changements importants dans ses jeux. Maintenant capable de constituer des images (symboles) dans sa tête, de les identifier (signifiant/signifié) et de les évoquer en l'absence de modèle, il élaborera tout un répertoire de jeux nouveaux, les jeux symboliques. Ces jeux vont évoluer du symbolisme individuel au symbolisme collectif selon des caractéristiques bien spécifiques et cela grâce aux nouvelles acquisitions sur les plans cognitif et social. 
L'enfant de cinq ans qui fréquente la classe de maternelle se situe, sur le plan de son développement, dans cette période fructueuse de la fonction symbolique. À cet âge, il appréhende le monde qui l'entoure par un mode symbolique qui se traduit fortement dans son activité ludique.

De plus, la classe de maternelle est un lieu où se regroupent au moins une vingtaine d'enfants chaque jour et ce, pendant plus de deux heures. Ce regroupement d'enfants dans un même espace entraîne un aménagement, une organisation de l'espace et un choix de matériel de jeu qui a pour but de favoriser le développement harmonieux de l'enfant. Ainsi, le rôle accordé à l'aménagement, à l'organisation matérielle du mobilier et au type de matériel de jeu privilégié pour stimuler, soutenir et confronter l'enfant dépasse dans ce contexte la simple interaction de l'enfant avec son environnement matériel. Cet environnement encourage et suscite de nombreuses interactions entre les enfants eux-mêmes et entre les enfants et l'adulte. Ce lieu, où le jeu de l'enfant est le plus considéré et le plus encouragé, crée des dynamiques sociales dû au seul fait que l'enfant est amené à partager avec les autres un espace et un matériel de jeu. Ces situations ludiques à plusieurs permettent aux enfants d'échanger, de discuter, de confronter leurs idées et leurs points de vue et de trouver ensemble un terrain d'entente pour mieux jouer et tenir compte de l'autre.

En classe de maternelle, l'enfant a donc la possibilité d'exercer et de mettre en valeur et à profit ce on joue dans diverses sphères d'activités, dans les différents coins et dans une multitude d'ateliers. Dans l'organisation de cet espace, le coin de la maison ou l'aire de la maison est sans aucun doute un témoin des plus reconnus et des plus évidents de la manifestation du jeu symbolique de l'enfant de cinq ans. En effet, l'organisation matérielle de cette aire de jeu et le matériel qui y sont proposés devrait être, selon un ensemble de critères bien définis, un lieu qui suscite des mises en scène variées permettant à l'enfant d'apprivoiser et d'expérimenter divers rôles parentaux et sociaux. L'aire de la maison constitue donc à cet égard un espace potentiellement riche pour la manifestation de l'activité symbolique.

Le mode symbolique d'appréhension du monde qui prédomine à cet âge, alimente et teinte aussi la majorité des actions et des interactions des enfants. Ce mode symbolique devient en quelque sorte la trame de fond sur laquelle se tisse le mode de développement 
de l'enfant de cinq ans et par conséquent la manifestation du jeu symbolique dépasse largement les possibilités offertes dans l'aire de la maison. Dans cette perspective, les possibilités symboliques ne sont plus restreintes au jeu qui se manifeste dans cette aire de la maison mais s'ouvrent dans les autres lieux d'activités de la classe maternelle. Partant de ces considérations, nous avons orienté l'exploration de ce on joue dans l'aire où l'on retrouve des blocs ${ }^{1}$. Car depuis l'intégration des maternelles dans le système scolaire public au Québec par le ministère de l'Éducation en 1964, les blocs ont toujours fait partie du matériel de jeu offert aux enfants. Encore aujourd'hui, dans la majorité $(90,5 \%)$ des classes maternelles (Thériault et al., 1987), on retrouve un coin bloc et du matériel de construction varié faisant appel à différentes techniques d'assemblage telles que les techniques de superposition, de vissage, de boulonnage, de chevillage et de collage. C'est sans doute par tradition qu'on réserve, dans les classes maternelles, un espace permanent où se retrouve ce matériel. Évidemment, la présence de cette aire de jeu et de ce matériel ne témoigne aucunement de son importance dans l'ensemble des interventions pédagogiques de l'enseignante et surtout dans l'activité de l'enfant avec le matériel bloc.

Dans ce lieu, l'enfant a l'occasion d'expérimenter tout un univers avec des blocs de bois vernis et nous croyons que le jeu est susceptible de s'exprimer d'une manière tout à fait originale dû en grande partie au potentiel même du matériel que sont les blocs et des activités qu'ils suscitent chez l'enfant.

L'observation faite lors d'une pré-expérimentation a révélé que l'espace réservé au bloc est souvent un espace contingenté, provisoire, séquentiel et souvent même délaissé, où les enfants ne s'attardent que rarement et pour de courtes périodes. Selon le cas, l'usage du matériel est réglementé de diverses façons. Certains enfants ne peuvent y accéder que durant la période de jeux libres, d'autres ne peuvent jouer avec les blocs qu'à partir de décembre ou qu'à la première moitié de l'année scolaire. Certaines enseignantes ${ }^{2}$ réservent un lieu pour ce matériel spécifique dans la classe, tandis que d'autres considèrent qu'on peut jouer avec les blocs sur la table. D'autres enseignantes

1 Dans ce texte, nous utiliserons les termes "coin bloc " ou "aire des blocs" pour définir le lieu ou l'espace où l'on retrouve le matériel bloc.

2 Dans ce texte, le terme enseignante sera utilisé comme épicène représentant l'ensemble des intervenantes œuvrant dans les classes maternelles en considérant que ces intervenantes sont en majorité des femmes. 
encouragent des projets individuels, tandis que certains enfants s'organisent en groupe pour réaliser une cons-truction collective, comme par exemple, une ville.

Viennent s'ajouter à ces constatations techniques et fonctionnelles sur l'aire des blocs, des observations concernant davantage la perception des enseignantes sur cette aire d'activités et sur ce matériel de jeu. Pour un certain nombre d'entre elles, les blocs sont perçus négativement. Ils sont considérés comme un matériel bruyant, utilisant beaucoup trop d'espace et par conséquent, l'accès à cette aire de jeu en est souvent limité et réglementé. Par contre, celles qui perçoivent positivement ce matériel lui reconnaissent un riche potentiel effectif susceptible de soutenir l'enfant dans l'une ou l'autre des différentes facettes de son développement, sans toutefois pouvoir identifier de façon spécifique ses contributions sur le plan développemental.

C'est trop souvent dû à des connaissances fragmentaires quant aux apports importants de ce matériel de jeu sur le développement de l'enfant, que l'on dénote un manque d'intérêt et un manque d'originalité dans l'exploitation de cette aire d'activité et du matériel qui s'y retrouve. Par conséquent, une attention très diffuse lui est accordée par rapport aux autres aires de la classe maternelle.

De ces différents éléments de la situation apparaissent donc nos préoccupations de recherche, soit l'identification de la contribution du matériel bloc au développement de l'enfant de cinq ans, à une période où son activité ludique est principalement fructueuse sur le plan symbolique et du rôle des interactions sociales dans cette dynamique de jeu.

L'étude de l'activité de l'enfant suscitée par le matériel bloc constitue la problématique de cette recherche qui s'inscrit dans une démarche de compréhension, s'articulant autour de deux questionnements: un questionnement matériel et environnemental et un questionnement structural et interactionnel.

Le premier questionnement engendre une réflexion à double volet: un volet qui porte sur les potentialités symboliques du matériel bloc et un autre, sur le contexte environnemental de l'organisation matérielle de cette aire d'activités dans la classe maternelle. 
Le deuxième questionnement entraîne l'examen de l'activité de l'enfant dans cette aire de jeu avec le matériel bloc, par son observation, sa description et son analyse. Centrée sur l'activité de l'enfant, cette étude veut mettre en évidence les potentialités du matériel, les processus et les produits de cette activité avec le matériel bloc afin d'identifier certains paramètres particuliers quant à la nature et la structure de cette activité.

Ces deux pôles de questionnement orientent donc les premiers jalons de notre question générale de recherche soit l'identification des différentes activités suscitées par le matériel bloc dans le contexte de la classe maternelle et la contribution potentielle de ce matériel et de ces activités au développement cognitif et social de l'enfant de cinq ans.

La démarche d'appréhension et de construction de l'objet de notre recherche s'inscrit dans un objectif de formation centré sur l'interdisciplinarité. L'interaction des différentes disciplines dans la compréhension de l'activité de l'enfant avec le matériel bloc se traduit sur des plans d'intersection épistémologique, théorique et méthodologique qui ont tenté d'élaborer des cadres référentiels et complémentaires visant graduellement une interaction de ces disciplines.

Sur le plan épistémologique, nous avons privilégié les fondements d'une épistémologie constructiviste et interactionniste génétique et une épistémologie socioconstructiviste et interactionniste où les confrontations individuelles et interindividuelles constituent les assises fondamentales des productions de connaissances.

Sur le plan théorique, l'approche piagétienne dans un paradigme consructiviste et l'approche vygotskienne et celles de Doise et Mugny (1981) et Perret-Clermont (1979) dans un paradigme socioconstructiviste ont constitué les choix théoriques des concepts relatifs à l'activité de l'enfant avec le matériel bloc.

Sur le plan méthodologique, l'approche observationnelle dans une méthodologie qualitative a nécessité l'investigation de différentes étapes d'observation selon des approches éthologique et anthropologique. Aussi, nous avons privilégié des modèles de mise en forme et d'analyse des données issues de l'analyse de contenu de L'Écuyer (1990) et de l'analyse de données de Huberman et Miles (1991). 


\section{CHAPITRE I}

\section{D'UN QUESTIONNEMENT MATÉRIEL ET ENVIRONNEMENTAL SUR LES BLOCS À UN QUESTIONNEMENT STRUCTURAL ET INTERACTIONNEL DE L'ACTIVITÉ DE L'ENFANT}

Le questionnement qui émerge de ce premier chapitre a pour but de montrer, d'une part, l'évolution du matériel bloc sur le plan industriel et, d'autre part, de faire ressortir les caractéristiques de ce matériel lorsqu'on le regarde comme objet de jeu, objet éducatif et pédagogique et objet de recherche.

Le chapitre fait une recension des écrits des quatre éléments mentionnés, montre la pertinence d'étudier ce matériel et conduit à la question de recherche qui s'actualise autour des potentiels possibles de ce matériel en rapport avec l'activité de l'enfant et des relations avec ses pairs en classe maternelle. Dans cette perspective, le titre de ce chapitre traduit l'investigation de deux pôles de questionnement: les blocs comme objet matériel qui se retrouve dans l'environnement éducatif et les blocs comme objet ayant un rôle structural et interactionnel de l'activité de l'enfant. 


\subsection{Vers une compréhension du matériel bloc et de l'activité qu'il suscite}

\subsubsection{Les blocs vus comme objet industriel}

Envisager les blocs comme objet industriel, c'est d'abord et avant tout les situer à travers l'histoire du jeu et du jouet, sous l'angle de sa fabrication et de son évolution matérielle.

Cette lecture historique fait donc ressortir l'évolution industrielle du matériel bloc selon les temps forts qui ont marqué la conception du jeu et du jouet et selon les influences dominantes de chacune des époques en matière d'éducation des jeunes enfants. Les différents pays qui ont contribué à la création de design de blocs, à leur mise en marché et à leur expansion seront aussi considérés. Cette démarche met donc l'accent sur les transformations graduelles du matériel bloc quant à la forme, aux matériaux et aux techniques d'assemblage exploitées. Car, comme le souligne Campagne (1989), le jouet témoigne de son époque, non seulement en nous donnant une idée de la maitrise technique de l'homme sur la matière, mais aussi en nous aidant à percevoir différentes façons d'inclure l'enfant dans la société ou de lui expliquer certains mécanismes du monde qui l'entoure.

C'est au XIXe siècle, aux États-Unis et en Europe, que s'établissent les premiers jalons d'une industrie qui deviendra florissante, celle du jouet, dans laquelle une large part est attribuée au matériel bloc. Les premiers blocs (1800) sont fabriqués en bois et laissent apparaitre sur chacune de leurs faces, des images d'animaux, des chiffres, des lettres de l'alphabet et des scènes d'enfants de la vie quotidienne.

Entre 1860 et 1870 , on retrouve sur le marché les "Hill's Alphabet Blocks". Dans un coffret en bois, Hill propose une série de blocs colorés et illustrés représentant sur chacune des surfaces du cube, une lettre de l'alphabet et un mot commençant par cette lettre. Ces blocs de bois peuvent être assemblés par une technique de juxtaposition et de superposition, pour former des mots ou la chaîne alphabétique. À la même époque, Hill s'associe à Hillduring et, ensemble, ils créent un important design de blocs reproduisant différents édifices tels que maison, garage, magasin général qui, par leur agencement, suggèrent la construction de villes et de villages. 
Vers 1876, toujours aux États-Unis, la famille Crandall se distingue en créant la première manufacture de blocs en bois. Elle met également au point une nouvelle technique pour l'utilisation de ce matériel, l'imbrication qui s'ajoute alors aux techniques de juxtaposition et de superposition. Une première forme d'imbrication se réalise à la manière des dents de peigne par Crandall en 1876 et une deuxième fait appel aux systèmes de rainures en 1881 et permet l'emboîtement des blocs les uns sur les autres. Ce système est demeuré l'un des plus populaires.

Simultanément en Europe, Ritcher en fabrique une brique en cast-stone appelée Ritcher Building. Ces briques sont de formes triangulaires, rectangulaires et rondes, de couleurs rouge, bleue et grise, et permettent des constructions détaillées et complexes de ponts.

Le $\mathrm{XX}^{e}$ siècle est, pour la société en général, une période de grands changements. Deux guerres et une multitude de découvertes scientifiques sont des preuves tangibles qui témoignent de plusieurs revirements de ce siècle. Inévitablement, la conception du jeu et du jouet suit ce mouvement et entraîne des transformations majeures dans la conception et la fabrication des jouets en général et des blocs en particulier.

La première moitié du XXe siècle cautionne les différents designs de blocs conçus jusque-là. Ainsi, au début du XXe siècle (1900), aux États-Unis, on voit apparaître sur le marché les Village blocks. Ces blocs sont en fait une combinaison des deux designs qui ont inspiré, jusqu'à cette date, les fabricants de blocs, soit les lettres de l'alphabet et les images d'édifices publics.

À la même époque, une nouveauté, et non la moindre, apparaît sur le marché américain. Ce sont des blocs réalisés par Caroline Pratt $(1933,1966,1984)$. Fruit d'une importante réflexion sur la philosophie de l'éducation préscolaire, cet ensemble de blocs en bois naturel vernis est composé d'une variété de formes: quadrangle, colonne, cylindre, demi-cercle, rampe, triangle, courbe elliptique, planche de toit, etc. (voir figure 1, appendice A.1). Ce matériel offre donc à l'enfant l'occasion d'exprimer ses besoins d'actions, sa connaissance et son rapport au monde qui l'entoure. 
La deuxième moitié du $\mathrm{XX}^{\mathrm{e}}$ siècle se révèle fort différente en matière d'expansion du matériel bloc. Deux types d'influence marquent cette période où des transformations majeures s'amorcent dans la conception du jeu et du jouet. Une première influence est caractérisée par l'arrivée de l'électricité, des deux guerres mondiales, du goût démesuré pour les constructions colossales, de la révolution industrielle et de la fabrication en quantité de jeux électriques, de construction, de miniaturisation et d'assemblage. Une deuxième source d'influence est la contribution plus qu'importante de la psychologie avec l'élaboration de théories du développement de l'enfant.

En ce qui concerne les blocs, la matière plastique et l'utilisation de colorant viennent révolutionner les procédés de fabrication et relèguent presqu'aux oubliettes le bois comme matériau de base. On assiste donc, à partir des années 50, à une effervescence dans la création de designs de blocs et dans l'invention et l'exploitation de différents mécanismes d'imbrication des blocs, engendrant des techniques d'assemblage variées et nouvelles.

C'est sans aucun doute le concept de briques, développé par Ole Kirk Christiansen en 1934, fondateur du groupe LEGO au Danemark, qui donne le coup d'envoi à une multitude de designs de blocs, tant en Europe qu'en Amérique. Chaque décennie à partir de 1950 propose des blocs qui sont, pour certains, semblables au concept LEGO ou, au contraire, différents avec des blocs aux formes et aux mécanismes sophistiqués, comme par exemple, ceux que l'on retrouve dans la série ASCO.

Dans l'orientation du présent projet, il est pertinent d'analyser l'évolution industrielle du matériel bloc. Cette analyse met en évidence les diverses possibilités de produire des structures différentes. La réalisation de ces structures ou de ces constructions est étroitement reliée au design donné à ce matériau. Effectivement, la gamme LEGO, avec ses petites et grosses briques de quatre à huit tenons et les autres blocs de même type proposant une technique d'emboîtement par superposition et juxtaposition, fournissent à l'enfant une infinité de possibles dans la création de productions dites ouvertes et figuratives. Avec ces blocs, l'enfant crée différents environnements tant imaginaires qu'inspirés du quotidien. Ces créations favorisent la mise en scène de situations personnelles et familières. Les blocs de bois de Pratt s'inscrivent également dans ce type de matériel permettant des productions dites ouvertes et figuratives. Cependant, étant donné leur 
taille, elles favorisent en plus l'investissement de l'enfant lui-même comme principal acteur dans ces environnements, sans intermédiaire de figurines. Cet investissement apporte au jeu un autre niveau de connaissances et de mises en scène.

D'autres designs de blocs suggèrent la construction de produits inusités et originaux stimulés à la fois par des agencements qui rejoignent les rangs de productions ouvertes et non figuratives. Entre ces deux extrêmes de productions, fruits de l'activité de construction, on retrouve des blocs à productions dites fermées et figuratives. Ces blocs, de par leurs pièces, leurs formes et leur nombre restreint, sont destinés à un seul type de production déjà imaginée par le fabricant, soit la construction de maisons et d'édifices, de bateaux, de véhicules et de camions. Ils sont souvent présentés dans des boîtes à thème (concept développé par LEGO, en 1964) et sont tributaires des grands événements de l'actualité ou inspirés de films et d'émissions pour enfants. À travers cette évolution, LEGO (1974) lance sur le marché les petits personnages et accessoires accompagnant les briques de construction. L'arrivée de ces personnages ouvre assurément des horizons intéressants sur le plan symbolique. À la fin des années 70 , les blocs subissent le même sort que l'ensemble des autres jouets et sont de plus en plus suggestifs, pour ne pas dire prescriptifs, dans l'orientation des productions dites fermées et figuratives. On en est presqu'au kit du parfait constructeur, avec plans et modèles de reproductions, oubliant, peut-être, que l'enfant a un cerveau et de l'imagination qu'il est capable de mettre à profit dans ses réalisations.

Dans les années 80 , le marché propose une multitude d'environnements du monde des adultes (jeux d'aventures air/terre, aviation, centre d'approvisionnement spatial, aéroport, voitures cosmiques) et quelques percées de nouveautés tentent de se frayer un chemin. Quelques fabricants proposent des types de blocs en plastique, en polyéthylène ou prolypepylène, permettant la construction de structures géantes et à la taille des enfants. Ces structures offrent diverses apparences de la maison, de meubles modulaires, de tours, de castelet et de cages à grimper, glisser et rouler.

Parmi tous ces designs de blocs, les ancêtres en bois suggéraient d'abord comme technique d'assemblage, la superposition, la juxtaposition et l'alignement. Depuis, les innovations n'ont cessé de croître. Des nouvelles techniques d'assemblage sont nées et on peut faire l'emboîtement par vissage, par superposition et par rainures ; par l'emboîte- 
ment de baguettes, l'enclenchement par glissement et le dégagement par torsion, le glissement des tenons et des rainures; par l'insertion de deux parties d'une charnière, l'insertion de boulons dans des trous, l'emboîtement à angles aigus, droits ou obtus, l'insertion avec des joints, l'emboîtement de goujons, la fixation de pièces trouées à des tiges flexibles en accordéon, l'assemblage par magnétisme, l'assemblage avec des goujons enfoncés dans des trous, l'imbrication de pièces dentelées sur les pourtours et les techniques de glissement par pression des doigts.

Dans cette vague de création de blocs en plastique, il y a eu, au cours des 30 dernières années, des tentatives quelques peu timides de retour aux sources, en ramenant sur le marché les blocs de bois. Les propositions des fabricants s'étendent des blocs conventionnels avec les lettres de l'alphabet en passant par ceux ressemblant aux blocs de Pratt, mais de plus petites tailles et de couleurs variées qui permettent toutes sortes de constructions, jusqu'aux blocs de formes variées (planches trouées avec boulons, blocs cylindriques avec larges rainures, formes diverses avec de petits goujons, baguettes et formes rondes en bois) qui donnent la possibilité de quelques constructions fermées et figuratives, comme des maisons et des objets familiers en faisant appel à des techniques d'assemblage de vissage et dévissage, d'emboîtement avec des baguettes et des petits goujons.

Récemment, sur le marché américain, on retrouve des blocs appelés les archiblocs en bois qui, par leur design, invitent les enfants à vivre des expériences architecturales représentant des éléments de l'architecture romaine, gothique et japonaise.

Finalement, durant les 50 dernières années, dans ce monde tumultueux du marché du jouet, les designs de blocs se sont multipliés, indiquant une évolution dans la réalisation de ce matériel. Les matériaux sont passés du bois au plastique, la coloration du naturel à la palette de couleurs et les techniques d'assemblage ont connu une gamme d'innovations. Malgré toutes ces nouvelles techniques d'assemblage, la technique d'emboîtement par superposition et juxtaposition demeure une technique simple des plus populaires et des plus exploitées par les fabricants. La technique d'empilage est aussi, pour les blocs de bois conventionnels et pour certains types de blocs en plastique, la seule technique utilisée. 
Dans une perspective industrielle, cette lecture du matériel bloc permet de le situer au rang d'objet qui a traversé l'Histoire avec une permanence et une immortalité tenaces (Campagne, 1989) puisque depuis le XIX ${ }^{\mathrm{e}}$ siècle, il n'a cessé de changer d'apparence, mais il est toujours présent. Il demeure toutefois un objet-jouet questionnant face à l'intérêt qu'il a suscité et qu'il continue de soulever autant chez les petits que chez les plus grands. Il a su maintenir au fil des époques cet enthousiasme autant de la part des enfants que des fabricants. Il continue encore aujourd'hui, dans les classes maternelles, à avoir une place et à captiver les enfants de longues heures lorsque les enseignantes leur en donnent l'occasion. Malgré cette évolution des formes et des matériaux, un type de blocs, celui de Pratt, a su garder une popularité et se retrouve dans la plupart des classes maternelles, tantôt dans un espace aménagé où les enfants, en toute liberté, ont le plaisir d'être ingénieurs; tantôt dans un coin remisé tant bien que mal, car on ne sait trop quoi en faire, mais qui soulève à la fois nostalgie et embarras quant à son exploitation. De plus, ce type de blocs se retrouve très rarement dans les salles de jeux des enfants à la maison, sans doute par économie d'espace de jeu et de rangement et en raison de son coût élevé, ce qui en fait un matériel à découvrir à la maternelle, tant au niveau de la matérialité que des possibilités d'expériences de jeu.

Toutes ces raisons font donc des blocs de bois de Caroline Pratt un objet à la fois ancien et contemporain qui mérite un regard réflexif sur ses potentialités et sur les activités qu'ils suscitent.

\subsubsection{Les blocs vus comme objet de jeu}

Envisager une recherche sur l'activité des enfants avec le matériel bloc, c'est alimenter une réflexion sur les blocs comme objet de jeu. Maints auteurs, en abordant le jeu des enfants, sont obligés, à un moment de leur démarche, d'ouvrir une parenthèse sur le jouet pour le définir par rapport au jeu et pour le situer dans le jeu. Cependant, à travers l'ensemble de ces définitions, deux points de vue nous ont guidée pour distinguer les particularités des objets de jeu.

Il existe deux sens au terme jouet. Un premier sens est lié étroitement au caractère éphémère d'un objet indéterminé qui, le temps du jeu, reçoit une signification particulière mais, une fois le jeu terminé, reprend son identité première telle que, morceau de bois, 
brin d'herbe, boîte de carton... "Le jeu est ainsi producteur de jouets, de ses instruments éphémères [...] il est produit par l'enfant, dans et pour son jeu." (Brougère, 1984a, p.80)

Un deuxième sens est associé au caractère permanent de l'objet, lié de façon intrinsèque à sa matérialité, puisque celui-ci demeure jouet, même en dehors du jeu. L'objet possède des caractéristiques internes et un potentiel propre. Au lieu de naître du contexte et des besoins du jeu, l'objet suscite le jeu qui devient le prolongement de l'usage de l'objet. Les blocs font partie de ce deuxième sens du terme jouet. Ils ont un potentiel latent intrinsèque qui se traduit à travers l'activité de l'enfant.

Communément, c'est du point de vue psychologique que le jouet ou l'objet de jeu a été et est défini. Celui-ci a pris de plus en plus de place depuis le XIXe siècle avec l'évolution des connaissances sur l'enfant et son développement, c'est-à-dire depuis l'essor de la psychologie de l'enfant. Dès lors, le terme jeu s'est infiltré peu à peu dans les activités de l'enfant jusqu'à sa reconnaissance comme activité principale, puisqu'il est devenu difficile de distinguer le jeu du non-jeu. Dans ce contexte, les jouets sont conçus et perçus comme des objets possédant des caractéristiques internes permettant de favoriser, de supporter et de faciliter les différentes facettes du développement de l'enfant. Les qualités du jouet sont directement reliées aux différentes valeurs issues du caractère développemental de l'objet de jeu. Selon les auteurs, on lui attribue une valeur expérimentale qui permet à l'enfant d'expérimenter, de manipuler; une valeur de structuration par un apport relatif à l'élaboration de sa personnalité; une valeur de relation où le jouet met l'enfant en rapport avec les autres et avec son environnement et une valeur ludique dans ce que le jouet apporte au jeu (Campagne, 1989, p.114). Ou encore, selon une autre classification, on lui accorde une valeur fonctionnelle qui permet le développement de la motricité; une valeur expérientielle laquelle s'attarde à développer les sens, la classification, la combinaison et les variations; une valeur formative qui permet à l'enfant d'explorer sa créativité et son imagination et une valeur relationnelle donnant du pouvoir au jouet en servant d'agent de liaison entre l'enfant et les autres enfants ou l'adulte (De Grandmont, 1989, p.81). Cette valeur de relation ou relationnelle pose toute la question du matériel bloc comme objet de liaison et objet d'interaction entre les enfants.

Du point de vue psychologique, que l'on parle du jouet éphémère ou permanent, il demeure une particularité de l'objet de jeu en tant qu'agent de développement des diffé- 
rentes facettes de la personnalité de l'enfant. Dans ce sens, l'objet de jeu doit être investi de valeurs développementales qui justifient en quelque sorte la qualité de l'objet et par le fait même, son choix.

Cependant, un point de vue social vient nuancer et compléter la définition et les dimensions du jouet. Peu souvent, nous réalisons une lecture de l'objet de jeu d'un point de vue social qui apporte des éléments complémentaires de réflexion sur des jouets, pensés, conçus et fabriqués par des adultes pour les enfants dans une société donnée.

Les travaux de Brougère (1984b) sont particulièrement intéressants et informants sur l'approche sociale du jouet qui permet de l'analyser comme média et médiateur entre les adultes et les enfants afin de percevoir quel message est contenu dans ces objets que les adultes conçoivent, construisent et transmettent à leurs enfants.

Ce point de vue social propose de définir le jouet comme média culturel, un objet support de représentations culturelles. Cette définition engendre deux directions dans la façon d'aborder le jouet. Une première direction identifie d'abord le jouet comme étant un support, un instrument de jeu qui donne les moyens d'accéder à certaines formes de jeux culturellement déterminées. Une deuxième direction identifie le jouet comme un moyen de transmission de contenus culturels. En fait, le jouet transmettrait simultanément à son utilisateur des règles de jeu, des contenus ludiques et des représentations sociales. Des règles de jeu qui s'inscrivent dans la dimension fonctionnelle même d'un jouet (la forme, la couleur, le matériau, l'aspect tactile, l'odeur, les bruits ou les sonorités émises qui incitent à une certaine manipulation, utilisation et action). Des contenus ludiques ancrés dans ce que l'objet représente ou évoque du monde réel ou imaginaire, ils sont suggestifs et imprègnent les premières orientations de jeu. Des représentations sociales qui mettent en évidence une fonction sociale du jouet qui renvoie à tout un système complexe et partiellement élucidé de représentations de l'enfant.

À la base de cette conception, le jouet est considéré comme un moyen de communication au même titre que le livre, la bande dessinée, le journal, la télévision, etc. qui s'insère dans un contexte social et culturel donné, donc imprégné des valeurs et des significations de notre société. 
En considérant le jouet comme un média (un média à trois dimensions), il prend place aux côtés de nombreux moyens de transmission culturelle dont dispose l'enfant et son analyse commande les mêmes traces que les autres médias, avant tout usage ludique en dehors du jeu. L'essentiel dans l'analyse du jouet réside dans la considération du message qui est transmis par ce moyen original de communication et ses conditions de transmission. Il se dégage donc deux questions importantes sous-jacentes à ce média. Quel message transmet-il? et Comment le fait-il?

Selon cette position sociale du jouet, notre culture, loin d'être homogène, est traversée par des courants divers qui engendrent des variations dans les messages inscrits en fonction de l'âge et de la diversité des représentations, tantôt complémentaires, tantôt concurrentes. Ce sont ces différentes visions qui ont inscrit, époque après époque, les dimensions ludiques éducatives, pédagogiques et développementales dans le jouet luimême. "Au travers du jouet, c'est toute la représentation de l'enfance et de son monde qui se donne à lire." (Brougère, 1984a, p.64)

Donc, quel message le jouet transmet-i1? C'est un message à double aspect qui est inscrit dans le jouet, car s'il transmet des possibles de jeu, il transmet des images qui proviennent d'une banque d'images propres à chaque culture. Par la suite, c'est avec ces images que l'enfant peut s'exprimer et c'est en référence à ces mêmes images qu'il peut saisir de nouvelles productions. (Brougère, 1987)

C'est à la fois sous un aspect statique et un aspect dynamique que s'inscrivent les divers sens culturels et sociaux. Ces deux aspects sont donc porteurs de significations, proposant une variété d'images. De plus, le jouet est constitué de deux dimensions importantes, la dimension matérielle et la dimension symbolique. Ces deux dimensions définissent à leur tour les aspects statique et dynamique du jouet. Le jouet, comme média culturel, propose aux enfants deux banques d'images importantes. Une première banque d'images provient d'abord du jouet lui-même comme entité propre, hors contexte de jeu, et constitue l'aspect statique du jouet. Une deuxième banque d'images naît du jeu puisque l'objet est destiné au jeu et la relation s'établit entre le jouet et l'enfant; cette deuxième banque d'images alimente à un premier niveau l'aspect statique et à un deuxième niveau l'aspect dynamique. 
Sous son aspect statique, le jouet est d'abord objet de conception, de fabrication et de consommation, dans notre société occidentale, du moins. Il porte, dans sa matérialité même de jouet, différentes significations culturelles, avant même qu'il se retrouve entre les mains des enfants. Il est, dès lors de sa conception par des adultes, par son matériau, sa forme, sa couleur et sa représentation externe, porteur de sens quant à la perception de l'adulte du monde de l'enfance; c'est ce que Brougère appelle la dimension matérielle du jouet qui laisse transparaître les premières traces des valeurs culturelles des adultes. Le jouet est en fait la matérialisation de valeurs culturelles et sociales.

Le jouet est constitué d'une autre dimension importante soit celle de la représentation ou de la dimension symbolique. Dès lors, le jouet redouble de significations puisque cette dimension symbolique s'actualise à deux niveaux de représentation. À un premier niveau, le jouet ou l'objet représente ou évoque quelque chose de réel dans l'environnement (voitures, animaux, accessoires domestiques, camions, etc.) ou une production imaginaire (héros, personnages et accessoires tirés de films, de bandes dessinées, d'émissions de télévision, etc.). À ce premier niveau, la dimension symbolique du jouet renvoie à des significations particulières de l'objet. Il propose, par ces différentes représentations, l'apprentissage du monde social, c'est-à-dire permet de reconnaître, de distinguer et d'apprivoiser les caractéristiques particulières et les tâches spécifiques associées à un rôle parental et un rôle social donné. En fait, le jouet est imprégné de l'image et des attentes que les adultes se font des enfants et de l'orientation que ces mêmes adultes désirent donner au jeu. Ce premier niveau de la dimension symbolique renvoie encore à ce que nous appelons l'aspect statique du jouet.

Sous son aspect dynamique, le jouet invite à des jeux symboliques; c'est le deuxième niveau de représentation de la dimension symbolique. Dès sa conception, le jouet transmet des possibles de jeu vers un destinataire potentiel privilégié, l'enfant. C'est à ce deuxième niveau que le jouet, média culturel, tire toute son originalité et sa distinction des autres médias. Car, il est dès lors un média en volume qui permet non seulement la lecture, le regard mais aussi la manipulation et l'action. Ce deuxième niveau de représentation de la dimension symbolique implique toute la relation de cet objet destiné au jeu avec l'enfant, soit le récepteur. L'enfant, par et dans ses jeux symboliques, appréhende une réalité, sa réalité, par la manipulation, l'appropriation et l'invention de nouveaux sens. Dépendant de la richesse du monde intérieur des enfants, certains ne dépas- 
seront pas les représentations proposées par le jouet, alors que d'autres iront nettement au-delà de ses représentations, parfois surdéterminées. C'est ce qui différencie le conditionnement culturel de la liberté et de la création. Le jouet est donc autant objet de conditionnement qu'objet de dépassement.

C'est à travers ce deuxième niveau de la dimension symbolique que le jouet prend tout son sens. Jusqu'ici objet statique, porteur de significations culturelles et sociales, on peut lui attribuer tous les qualificatifs et les déterminants inimaginables, ceux-ci demeurent des possibles et des représentations d'adultes pour les enfants prisonniers dans la matérialité du jouet. Mais la question qui se pose: Qu'est-ce que l'enfant fait avec cet objet ou ce jouet? Avec cet objet préalablement défini et codé culturellement, deux voies possibles s'offrent à lui. Ou bien, il joue avec l'objet selon l'orientation et les attentes des adultes concepteurs, fabricants et consommateurs, et c'est là que l'inscription des déterminants culturels s'actualise au maximum. Ou bien, l'objet, au-delà des déterminations inscrites, devient le tremplin à une intériorisation personnalisée des contenus et des comportements. Car l'action (comportement ou discours) de l'enfant dépasse la simple imprégnation des contenus symboliques et devient, à ce niveau, appropriation active, individuelle des contenus et réinterprétation de ces codes par l'enfant et ainsi, reconstruction de sens et de nouvelles significations à travers le processus dynamique de cette relation enfant-jouet.

Dans le cadre de cette étude, le point de vue social fournit des indices quant à une possible empreinte sociale que porte déjà l'objet en lui, dans sa dimension matérielle et surtout dans sa dimension symbolique. Dans sa dimension matérielle, les blocs de Caroline Pratt en érable vernis proposent différentes formes qui invitent d'abord à une activité de construction. Communément, ces blocs guident la réalisation de différents genres d'édifices (maisons, châteaux, garages, hôpitaux), de divers moyens de transports (bateaux, avions, camions, autos, traversiers) et de systèmes routiers variés et complexes. Sans l'intervention de l'enfant, les blocs eux-mêmes demeurent des formes simples et sans représentation. Par leur matérialité, les blocs orientent déjà le genre de productions que les enfants peuvent réaliser.

Dans sa dimension symbolique, l'intervention de l'enfant prend un autre sens, son sens. À un premier niveau de représentation, l'enfant, à partir de ces différentes 
formes de blocs, peut représenter autant d'univers familiers que d'univers imaginaires, autant d'univers déjà imprégnés dans la matérialité des blocs eux-mêmes. À un deuxième niveau de représentation, toutes les productions réalisées par les enfants peuvent devenir un élément moteur et déclencheur autour desquelles vont s'élaborer une infinité de jeux symboliques. Avec le matériel bloc, l'enfant est d'abord constructeur et ensuite scénariste et acteur. C'est donc l'interaction de l'enfant avec les blocs et l'interaction des enfants entre eux qui donnent lieu à l'expression de nombreuses significations. L'enfant manipule donc, à travers ce matériau, de nombreuses images allant de la représentation d'objets, à la transposition de lui-même et de son vécu jusqu'à l'investissement de personnages et d'événements familiers et imaginaires.

\subsubsection{Les blocs vus comme objet éducatif et pédagogique}

Envisager les blocs comme objets éducatif et pédagogique, c'est encore situer ce matériel à travers l'histoire du jeu et du jouet, sous l'angle de l'éducation des enfants, mais cette fois-ci, en regard de sa contribution aux philosophies et aux méthodes d'éducation du jeune enfant. Cette lecture historique met en évidence les temps forts qui ont marqué la conception du jeu et du jouet selon les influences dominantes de chacune des époques en matière d'éducation des enfants. Ces influences ont probablement imprégné les conceptions du jeu et du jouet et donné naissance successivement, à travers les époques, aux dimensions ludique, éducative, pédagogique et développementale du jeu et du jouet.

Il faut remonter à la fin de la Renaissance pour voir apparaître les premières traces de la dimension éducative du jeu. Mais c'est au XVII ${ }^{e}$ siècle, marqué par de profonds changements de la conception du jeu et du jouet, que le jeu éducatif sera spécifiquement conçu et produit à des fins pédagogiques. Inévitablement, les jouets qui soutiennent le jeu des enfants sont qualifiés des mêmes attributs et les objets deviennent éducatifs et servent, eux aussi, à des fins pédagogiques.

Le XIX ${ }^{e}$ siècle, pour sa part, est une période de consolidation de ces positions pédagogiques du jeu. Il est également une période florissante en matière d'élaboration de modèles d'éducation du jeune enfant, parfois inspiré d'un matériel éducatif comme les blocs, et imprégné essentiellement de ces visées éducatives. 
Finalement, le $\mathrm{XX}^{\mathrm{e}}$ siècle est marqué par deux temps forts dans la conception du jeu. La première moitié de ce siècle continue à perpétuer la dimension éducative du jeu et par le fait même du jouet, seule dimension honorable d'ailleurs pour sa reconnaissance. La deuxième moitié est porteuse de transformations majeures dans la conception des jeux et des jouets.

Avec l'essor incontesté de la psychologie et des nouvelles théories sur le développement de l'enfant dans les années 50 , le jeu obtient un autre titre de noblesse, celui d'outil de développement. Le contexte social de cette période entraîne une nouvelle avenue pour les jouets. Ils peuvent désormais faciliter, favoriser et supporter certains apprentissages dans les différentes étapes du développement de l'enfant, sans toutefois perdre de vue qu'ils ne remplaceront jamais l'action et la pensée de l'enfant. Dans le même sens, les divers systèmes pédagogiques reflètent toujours, eux aussi, plus ou moins l'époque où ils ont vu le jour. Ceux qui ont été élaborés à la fin du XIX ${ }^{e}$ siècle et au début du $\mathrm{XX}^{\mathrm{e}}$ proposent une multitude de méthodes pédagogiques.

Une lecture historique des philosophies et des méthodes d'éducation des théoriciens et pédagogues qui ont laissé leurs empreintes aux diverses conceptions de l'éducation préscolaire en général et, plus près de nous, à l'éducation préscolaire au Québec, permet de mettre en évidence ce matériel, les blocs, comme élément important, voire même essentiel, dans l'élaboration de modèles d'éducation et dans la création de programmes pour les enfants de cet âge. Le domaine de l'éducation préscolaire est sans aucun doute le domaine qui a su le mieux réinvestir à profit le matériel bloc. Cette lecture démontre que les blocs ont servi de support à l'opérationnalisation des principes de base sous-jacents ces philosophies et ces modèles, et qu'ils ont été un matériel de base privilégié dans la conception de certains programmes d'éducation préscolaire.([Edgeworth, 1790; Froëbel, 1826; Locke, 1690; Montessori, 1912; Pratt, 1914: voir Provenzo et Brett, 1983], Dodge, 1979a; Foster, 1978).

C'est seulement dans la deuxième moitié du XIX ${ }^{\mathrm{e}}$ siècle que les réflexions de certains pédagogues s'intéressant aux blocs se concrétisent dans un matériel s'insérant dans un ensemble de moyens pédagogiques. Déjà au début du XVIIre siècle, les réflexions de Locke, dans ses propos sur l'éducation, discute des différents jouets éducationnels et indique que les plus intéressants semblent être les blocs alphabet. Vers la fin du même 
siècle (vers 1790), Edgeworth et Lovel Edgeworth considèrent les blocs comme un jouet qui développe "l'exercice du sens et de l'imagination, de l'imitation et des pouvoirs inventifs."

Une analyse plus raffinée du matériel éducatif en général et du matériel bloc en particulier, fait ressortir trois éléments importants qui s'inscrivent dans la problématique de la présente recherche.

Le premier élément repose sur des principes généraux qui s'intègrent dans une vision fondamentale et particulière du développement du jeune enfant. Ces principes s'articulent pour chaque théoricien dans l'élaboration d'une philosophie ou d'une méthode d'éducation du domaine préscolaire. Par la suite, ces mêmes principes sous-tendent la conception d'un matériel opérationnalisant ainsi la philosophie ou la méthode. Pour chacun des théoriciens concernés, il semble bien que le développement de l'enfant global et/ou harmonieux soit un but à atteindre. Deux facteurs constitutifs de ce développement se dégagent: l'importance des sens et l'importance des moyens d'action de l'enfant.

Le deuxième élément qui apparaît essentiel dans ces différentes philosophies ou méthodes, c'est la conception du jeu et du jouet. En effet, pour Froëbel, Séguin et Decroly, le développement de l'enfant se fait à travers le jeu. Froëbel, en particulier, souligne que c'est par le jeu que l'enfant comprend le monde, qu'il cultive son imagination, entraîne ses structures mentales et que le jeu est avant tout procédé d'éducation, c'est-à-dire l'élément propre à éveiller l'intelligence et l'affectivité. Séguin, pour sa part, ne limite pas le rôle du jeu à l'éducation intellectuelle, mais assigne aux jouets "la part principale dans le développement des sympathies humaines." Il analyse longuement par quels processus le jeu et les jouets contribuent à la formation de l'enfant. Il faut comprendre comment les jouets peuvent servir à l'éducation, car “[...] les jouets sont des moyens intermédiaires d'expériences entre la grande réalité de la vie et la faiblesse de l'enfant." Enfin, Decroly rapporte que le jeu est le pont qui relie l'enfant à la vie par-delà le mur de l'école. Seule Montessori ne partage pas cette conception du jeu liée au développement puisque, pour elle, tous les enseignements sont orientés, laissant pour contre l'expression spontanée, la rêverie et l'imagination qu'elle considère comme des 
déviations et des fuites de l'énergie psychique. Le jeu est conçu, pour Montessori, comme vide de sens.

Un troisième élément concerne la conception ou l'adoption d'un matériel qui découle des différents principes de base de la philosophie ou de la méthode. Ce matériel opérationnalise de façon tangible les principes théoriques qui sous-tendent les différentes approches pédagogiques privilégiées. Plus près de notre objet de recherche, un intérêt marqué pour les blocs se dégage des enseignements privilégiés par Froëbel (les dons $3,4,5,6$ ), Séguin (les blocs dans l'enseignement de l'imitation personnelle), Montessori (la tour rose croissante et décroissante) et Decroly (l'utilisation des blocs pour l'activité motrice et le jeu de construction). Un des premiers enseignements qui semble essentiel pour chacun, c'est l'éducation relié au sens. L'éducation sensorielle est donc à la base de la conception du matériel et/ou de l'utilisation première du matériel afin de favoriser d'abord la manipulation, l'action et/ou les travaux manuels sur lesquels s'édifierait l'activité intellectuelle, à partir des rapports que l'enfant établit entre les choses et les moyens d'actions sur les choses (Froëbel, Séguin, Decroly), ou sur une généralisation des éléments sensoriels à toutes les activités (Montessori).

Le début du XX' siècle marque l'amorce d'un autre mouvement, celui de l'élaboration de programmes et de curriculum basés essentiellement sur le matériel bloc.

Aux États-Unis, ce mouvement débute en 1914 avec Pratt, chef de file dans l'éducation des jeunes enfants. À la suite de nombreuses observations des comportements des enfants dans sa pratique quotidienne d'éducatrice, elle a développé une philosophie de l'éducation préscolaire, qui manifeste une certaine révolte contre les méthodes traditionnelles d'enseignement et une recherche de significations de l'apprentissage. Elle tentait de trouver une formule originale, un concept nouveau d'éducation qui permettrait l'exploration du monde intérieur des enfants afin de découvrir des avenues potentiellement riches par rapport au développement et à l'apprentissage des enfants. Pour elle, le germe sérieux de l'apprentissage se trouve dans le processus du jeu de l'enfant. C'est ainsi qu'une première réflexion sur les concepts d'apprentissage et d'enseignement a engendré une réflexion sur le matériel de jeu. 
Le matériel bloc de Pratt est donc soutenu par cette philosophie d'éducation du jeune enfant. Pour la traduire en moyen d'action, elle développe un programme avec le matériel bloc. Ce matériel flexible s'adapte au besoin d'agir du corps de l'enfant, de son intelligence et de son affectivité. Par ce matériel, l'enfant a l'opportunité d'explorer une infinité et une variété de formes et de figures. Pour elle, ce matériel est complet en luimême puisque l'enfant peut transformer chaque pièce de bois au gré de son imagination. Le matériel de Pratt, tel que vu précédemment, sont des blocs en bois naturel, à grosseurs multiples d'une unité de base $(3,5 \times 7 \times 14 \mathrm{~cm})$ et de formes variées (module, quadrangle, colonne, cylindre, demi-arche, triangle, courbe elliptique, carrefour, chaussée, planche de toit). Sa philosophie repose sur trois points majeurs et huit principes de base qui mettent en évidence l'importance et la place du jeu, en général, dans la vie des enfants et l'importance et la place du matériel bloc, en particulier, comme moyen de développement.

Cette auteure considère le jeu comme un processus de développement dans lequel et par lequel l'enfant apprend à exprimer et à organiser ses différentes expériences de vie et ce, par l'intermédiaire d'un matériel qui s'adapte autant à l'extériorisation de sa fantaisie que de sa réalité. Le jeu reflète aussi le besoin d'autonomie et d'action de l'enfant dans son processus de développement.

Le matériel qui semble correspondre le plus à l'opérationnalisation de la philosophie de Pratt est un matériel qui donne à l'enfant le pouvoir de négocier avec l'environnement et ce pouvoir s'accroît avec l'utilisation libre d'un matériel constructif. Les blocs sont un matériel qui offre des possibilités similaires à un matériel d'expression du rythme, de modèle ou de design et ils sont comparables à la palette de couleurs de l'artiste-peintre. Finalement, le matériel bloc permet à l'enfant de revoir, de répéter et de revivre une multitude d'expériences.

Dans la même lignée, à la fin des années 1970, le matériel bloc est une fois de plus réinvesti dans l'éducation préscolaire. Dodge (1979a) et Foster (1978) élaborent des programmes d'éducation préscolaire basés sur ce matériel et ses diverses potentialités. Ces programmes sont réalisés dans un but de former du personnel travaillant auprès des jeunes enfants, les éducateurs et les enseignants, et proposent des ateliers de formation à 
partir des diverses voies possibles d'exploitation du matériel bloc et de ces nombreux apports, eu égard au développement de l'enfant.

A Creative Curriculum for Early Childhood Blocks, développé par Dodge et son équipe (1979b), met une emphase particulière sur l'utilisation de l'espace et de ce matériel dans la classe. Ce programme a pour but spécifique d'aider les éducateurs et les enseignants à identifier et à réfléchir sur les blocs et sur l'aire des blocs dans la classe tant au niveau des potentialités et des ressources inexploitées du matériel, de son organisation et de l'espace que du rôle de l'enseignant.

Foster (1978), dans The Blocks in the Early Childhood Program: Arizona, présente une démarche de questionnement et de réflexion sur le matériel bloc. Elle propose une série d'objectifs à réaliser par rapport aux mêmes préoccupations du précédent programme pour ce matériel. C'est en quelque sorte un manuel-guide servant à l'entraînement du personnel et à la sensibilisation sur ce matériel pour le développement de l'enfant.

Cette lecture historique permet d'apprécier l'intérêt, l'importance et la place privilégiés des blocs ayant servi à alimenter des réflexions sur l'éducation, puis à concrétiser des principes à la base des différentes philosophies et/ou méthodes et finalement, d'élaborer des programmes d'éducation préscolaire.

Ce survol met à jour un ensemble d'éléments qui ne fait que rendre encore plus actuelle notre problématique de recherche puisque les blocs, en traversant différentes époques, ont revêtu certes diverses apparences tributaires de ces mêmes époques mais demeurent encore aujourd'hui, un matériel riche en potentiel offrant plusieurs sources d'exploitation, moteur même de notre questionnement.

\subsubsection{Les blocs vus comme objet de recherche}

L'analyse du matériel bloc vu comme objet de recherche met en évidence l'évolution des champs d'intérêt des chercheurs. Les préoccupations théoriques et méthodologiques autour de ce matériel sont influencées et teintées par les divers courants de recherche qui prédominent. Ainsi, la production des connaissances se réalise dans les pos- 
sibles et les limites imposés par une vision particulière qu'engendre un courant de recherche privilégié et une approche préconisée. Deux perspectives importantes ont marqué et orienté la recherche sur le matériel bloc: la perspective psychologique et la perspective sociale.

\section{La recherche sur les blocs selon une perspective psychologique}

L'ensemble des études qui s'inscrivent dans la perspective psychologique se regroupent selon trois approches. Il s'agit des approches psychodynamique, développementale et pédagogique.

La première approche regroupe les études qui ont emprunté, au niveau théorique, des éléments à la psychodynamique dans laquelle s'inscrivent la théorie psychosexuelle de Freud $(1938,1962,1982)$ et la théorie psychosociale de Erikson (1982). L'approche développementale réunit l'ensemble des travaux qui ont tenté de saisir l'évolution de l'activité de construction en terme d'étapes ou de stades d'évolution, selon différentes données reliées aux produits de la construction. Finalement, l'approche pédagogique regroupe les études qui ont réinvesti le matériel bloc dans les différentes disciplines et didactiques. Ces approches font ressortir une préoccupation développementale importante accordée au matériel bloc, en mettant le focus sur l'acquisition et la consolidation de diverses notions et concepts relatifs à une discipline donnée et ce, à des fins pédagogiques.

\section{L'approche psychodynamique}

Erikson (1940-1951) est sans doute un porte-parole important des recherches sur le matériel bloc puisque son nom est associé aux plus anciennes études entreprises dans ce domaine. Ses travaux mettent en évidence les types de constructions réalisées par des garçons et des filles âgés de 11-12 et 13 ans, à qui on demandait de créer une scène excitante, imaginaire et originale à partir de blocs et de différents accessoires tels que des personnages, des animaux et des voitures. Tous les enfants ont construit des scènes issues directement de leur imagination. Les résultats obtenus ont été interprétés dans un cadre psychanalytique, relié à la théorie psychosexuelle de Freud et au concept de symbolisme psychosexuel. 
Dans ces études, les différences de construction, quant à la nature des productions des garçons et des filles, sont attribuées aux différences morphologique et fonctionnelle des sexes. Ces différences sont largement déterminées, selon Erikson, par les différences psychosexuelles et non par l'influence culturelle. Cet auteur, dans l'interprétation de ces données, considère la maturation sexuelle à l'origine des différences dans les constructions. De plus, les scènes représentées sont compatibles avec la théorie psychanalytique du développement psychosexuel qui met en évidence le processus par lequel des enfants commencent à devenir conscients de leur sexualité et ce, en construisant des scènes représentant leurs propres sexes. Dans ce sens, les garçons ont construit des rues et des scènes extérieures impliquant des accidents de voitures avec des animaux, des indiens et avec des jouets représentant le mouvement. On observe donc, chez les garçons, plus de structures en hauteur et de tours entourant ses constructions avec des objets en mouvement. Selon les résultats, les garçons utilisent plus de blocs que les filles et ce, d'une manière plus variée. Les filles, pour leur part, construisent régulièrement des scènes de la vie quotidienne de la maison et de l'école, impliquant une utilisation peu complexe des blocs ou encore, elles utilisent les personnages et les accessoires, sans les blocs. On observe, chez les filles, plus de salles, d'enclosures, d'ouvertures, d'embrasures et d'entrées dans leurs constructions.

Suite à ces études, il faudra attendre les années 70 pour que resurgisse dans les recherches sur les blocs la problématique des différences de constructions entre les garçons et les filles. Les chercheurs préoccupés par les variables de sexe (garçon et fille) et par le groupe d'âge (six ans; pré-adolescent, 11-12-13 ans; adolescent, 16-17 ans) ont retenu, sur les plans théorique et méthodologique, sensiblement les mêmes présupposés et les mêmes critères que ceux de Erikson. Les résultats obtenus dans l'ensemble de ces recherches sont similaires aux études antérieures avec, quelquefois, accentuation de certains critères ou attributs étudiés. Cependant, une divergence apparaît dans le cadre d'interprétation de ces résultats, divisant les chercheurs en deux groupes d'appartenance.

Un premier groupe de chercheurs (Blackman, 1977; Cramer et Hogan, 1975; Schuster, 1973 et Wambach, 1974) a adopté la même position que Erikson en considérant une interprétation psychosexuelle des données, mettant en relation les différences de constructions des garçons et des filles et les aspects morphologique et fonctionnel des différences anatomiques de ces derniers. 
Un deuxième groupe de chercheurs (Clance, Dawson et Gill, 1975; Wilcox, 1979) reconnaît que l'âge et le sexe sont des facteurs significatifs dans le jeu de construction mais préfère envisager les données obtenues à travers un point de vue de l'apprentissage social. Ils rejoignent ainsi une position plus orientée vers le processus d'établissement de l'identité personnelle et de l'apprentissage d'un rôle social et culturel en accord avec les attentes et les pressions sociales reliées à ce rôle. Cette interprétation s'inscrit dans la théorie psychosociale du développement de la personnalité élaborée par Erikson.

Finalement, en 1982, Goodfader entreprend une étude sur les différences de construction entre les garçons et les filles d'âge préscolaire selon trois théories majeures qui peuvent expliquer l'acquisition des rôles reliés au sexe: la théorie psychanalytique de Freud $(1938$, 1962, 1982), la théorie de l'apprentissage social décrite par Bandura et Walters (1963) et Mischel (1970) et la théorie cognitive du développement de Kohlberg (1966).

Dans son étude, Goodfader (1982) indique que les résultats mettent en évidence l'opportunité de continuer à considérer l'interprétation psychosexuelle dans les différences de construction des garçons et des filles, mais il importe de les examiner dans un contexte de complémentarité et d'enrichissement. Une interprétation dans le cadre de l'apprentissage social, pourrait expliquer comment les différences de socialisation entre garçons et filles se cultivent et se perpétuent selon des normes sociales établies en rapport aux différents rôles reliés à chacun des sexes.

\section{L'approche développementale}

Les premières recherches avec le matériel bloc axées sur l'approche développementale avaient pour but l'élaboration d'une échelle développementale de données en fonction de l'âge des enfants. Cependant, selon les résultats obtenus, cette idée fut rapidement abandonnée (Bailey, 1933). Par la suite, des études ont tenté de mettre en évidence différentes étapes d'évolution dans la réalisation d'une production avec le matériel bloc. L'identification de ces étapes est tributaire de plusieurs critères: l'âge de l'enfant, les habiletés et les procédés de construction, les notions de structuration de l'espace, la complexité des structures, etc. (Johnson, 1974; Moyer and Gilner, 1956; Reifel and Greenfield, 1982; Reifel, 1984; Robinson, 1958; Schirrmacher, 1975). 
De ces différents travaux, c'est Johnson (1974) qui a le mieux identifié certains procédés de construction propres à l'utilisation de ce matériel. Elle considère les blocs de construction comme un médium au même titre que la peinture et l'argile. Ce matériel fournit à l'enfant la possibilité d'exercer différents procédés de construction et de démontrer ainsi leur versatilité. Ce matériel permet également l'expression des idées et des sentiments des enfants. Pour Johnson, la richesse de ce matériel et des ressources imaginatives des enfants ne sont pas suffisamment prises en considération. Son étude répond à certaines questions: existe-t-il différents procédés communs à tous les enfants dans l'utilisation du matériel bloc? Est-ce que ces procédés correspondent à des étapes ou stades d'évolution dans la construction? Est-ce que ces étapes ou stades correspondent à des âges spécifiques? Quels sont les traits distinctifs de ces étapes ou stades d'évolution?

Ainsi, les nombreuses observations d'enfants de deux à six ans dans leur activité de construction avec le matériel de Pratt et les croquis réalisés par les observateurs (enseignants et étudiants) des différentes productions achevées traduiraient des stades ou étapes d'évolution spécifiques à l'activité de construction.

Dès l'âge de deux ans jusqu'à six ans, l'enfant passe à travers six étapes ou stades d'évolution dans sa construction avec le matériel bloc.

À son premier contact avec ce matériel, les diverses explorations et les manipulations des enfants sont ses premiers modes de connaissance. L'enfant transporte, empile et entasse les blocs mais ne réalise pas de véritables constructions. Par la suite, il acquiert quatre procédés de base qui sous-tendent ces premières constructions: la tour, la rangée, le pont et l'enclosure. Ces premiers procédés constituent et demeurent la base de toutes les constructions qui deviendront de plus en plus détaillées et complexes, grâce à la combinaison des procédés, à la complexité des détails, à la symétrie, à l'équilibre, etc.

À ces différents procédés de construction viennent s'ajouter des principes qui caractérisent chacun des stades: la répétition du procédé de base, la tendance à nommer les constructions, la représentation et le jeu symbolique.

La répétition d'un même procédé signifie que l'enfant consolide des savoir-faire en construction et qu'il est capable de les utiliser avec aisance. Ainsi, l'enfant passe à un 
stade plus avancé lorsque l'on observe, dans une production, la répétition du même procédé et la combinaison de différents procédés acquis. Les stades 2,3 et 4 élaborés par Johnson mettent en évidence l'apprentissage des quatre procédés de base qui seront ultérieurement utilisés dans les constructions des enfants. Le principe de répétition du nouveau procédé est l'indicateur de la consolidation de ce procédé. Le deuxième principe observé se manifeste par la tendance à nommer les constructions réalisées et par la tendance à répéter le procédé acquis en complexifiant les détails. Ainsi, une fois les procédés de base acquis (la tour, la rangée, le pont et l'enclosure), l'enfant peut créer des constructions plus élaborées aux niveaux des détails et de la complexité car son énergie n'est plus canalisée vers la maîtrise d'un procédé.

Au stade 5, les modèles décoratifs apparaissent et des constructions inusitées prennent forme. Ces dernières sont tout à fait remarquables par les détails, la symétrie, l'équilibre et l'originalité. À ce stade, la répétition des particularités des designs est très présente. C'est un stade qui met en évidence les arrangements, les organisations, les élaborations, les créations et les compositions des enfants. Cependant, les constructions sont rarement nommées. Si elles le sont, c'est surtout par imitation ou obligation, soit par l'exemple des plus grands qui nomment leurs constructions, soit parce qu'un autre enfant fait une remarque ou lui demande ce qu'il a construit, ou soit parce que l'adulte le questionne sur ce qu'il a construit.

Au stade 6, la tendance à nommer les constructions est de plus en plus présente. L'enfant annonce souvent au début de l'activité ce qu'il désire construire. Cependant, au début, cela ne signifie pas nécessairement que les constructions ressembleront à ce qui a été annoncé; par la suite, le nom des productions est davantage lié aux fonctions des constructions réalisées. À ce stade, les constructions reproduisent ou symbolisent des structures connues ou des expériences vécues. Un autre élément est alors mis en lumière; on voit apparaître une forte impulsion au jeu dramatique autour et à partir des constructions de blocs. Les blocs deviennent un cadre de référence dans lequel s'élabore le jeu dramatique qui devient coopératif et interrelié entre les enfants. La qualité réelle du jeu dramatique à partir des constructions n'est pas nécessairement due à la connaissance approfondie des procédés mais plutôt à l'attitude de l'enfant à l'égard du matériel. Ce matériel se prête à de multiples transformations. Enfin, Johnson souligne que même en identifiant des périodes spécifiques de l'évolution dans la construction, le rythme, les em- 
phases de chaque stade et les diverses voies d'utilisation que l'enfant est susceptible d'emprunter, varient en fonction de l'individualité de chaque enfant.

De plus, le rôle de l'adulte dans cette activité de construction est important. L'intérêt authentique manifesté par l'adulte à l'égard de la construction de l'enfant est un puissant facteur qui contribue à l'utilisation créative des blocs. C'est un matériel qui offre une multitude de possibilités à travers lesquelles les enfants peuvent revivre soit des expériences reliées au quotidien de la maison, de l'école ou d'ailleurs. Les propres réactions des enfants à l'égard de leurs constructions renseignent l'adulte sur leur intérêt, leur concentration, leur absorption, leur exaltation et leur enthousiasme vis-à-vis cette activité. Selon Johnson, la prudence des adultes par rapport aux suggestions et aux questions qui s'adressent aux enfants est de mise, parce que dépendant de l'étape dans l'évolution de la construction, les enfants ne sont pas nécessairement prêts à répondre, ou ne voient pas nécessairement la pertinence de ce questionnement.

Toujours dans cette perspective développementale, d'autres auteurs reconnaissent différentes étapes d'évolution dans l'activité de construction. Cependant, même s'ils proposent d'autres taxinomies des stades d'évolution, ils s'inspirent tous des quatre procédés de base et des étapes d'évolution identifiés et élaborés par Johnson.

Les travaux de Santa (1980) s'inscrivent dans la même lignée des travaux de Johnson. Elle identifie huit stades d'évolution de la construction, mais les deux derniers se distinguent de ceux identifiés par Johnson. Ces deux stades sont la capacité des enfants à reproduire des modèles et des plans (stade 7) et la capacité à esquisser les plans des productions et à concrétiser des plans par le biais du matériel bloc (stade 8). Voici comment Santa définit ces deux stades.

Au stade 7, reproduction de modèles et de plans, les constructions des enfants sont reconnaissables et ressemblantes. Elles sont qualifiées de véritables reproductions architecturales et sont tributaires des expériences architecturales vécues par les enfants. Elles sont le fruit de nombreuses discussions entre les enfants à propos des modèles qu'ils ont visités et des images et photos qu'ils regardent. Ainsi, les constructions telles que le monument Washington, la Maison blanche, The Washington National Cathedral et le Kennedy Center apparaissent régulièrement dans les constructions des enfants parce 
qu'elles ont été rendues visibles et familières. À ce stade, il arrive fréquemment que les enfants étiquettent certains travaux avec des noms et des signes variés.

Le stade 8 (vers six-neuf ans) a été élaboré à la suite d'une expérience avec le matériel bloc au Learning Center avec des enfants âgés de six à neuf ans, dans une classe du primaire. Il consiste, dans une première phase, à réaliser des plans de la construction sur papier et, dans une deuxième phase, à concrétiser ces plans avec le matériel bloc.

Dans la même ligne de pensée, Apelman (1984) propose une classification des stades d'évolution de la construction basée sur celle de Johnson, selon sept étapes ou stades. Elle identifie les mêmes stades que Johnson; par contre, le stade de la représentation est divisé en deux étapes d'évolution distinctes: l'identification et la représentation. Ainsi, au stade 6, l'enfant commence à nommer ses constructions en rapport ou non aux fonctions de la construction, pour ensuite, au stade 7, aborder le jeu dramatique. Il atteint le stade de la représentation lorsqu'il reproduit ou symbolise les constructions qu'il connaît et qu'un jeu dramatique plus organisé s'articule autour de la construction.

Dodge (1979a), l'auteure de Blocks: A Creative Curriculum for Early Childhood, inspirée des travaux de Johnson, regroupe les différents niveaux d'évolution de la construction en quatre stades spécifiques.

Finalement, Reifel (1984), en s'inspirant toujours des travaux de Johnson, fait ressortir les différentes notions spatiales impliquées dans l'évolution de la représentation spatiale chez l'enfant et ce, par le biais des différentes constructions qu'il réalise. Ainsi, à chaque procédé observé par Johnson, Reifel identifie les différentes notions et relations spatiales qui peuvent être mises à contribution.

Empruntant des éléments théoriques au cadre piagétien (Piaget et Inhelder, 1966) en ce qui concerne l'identification et la description des notions et relations spatiales de proximité (sur, au-dessus, par, à côté de), de séparation (près, dehors) et d'enclosure (à l'intérieur, à l'extérieur), Reifel, avec le matériel bloc, élabore trois stades d'évolution de la représentation spatiale et ce, dans le contexte de la représentation symbolique à partir du matériel bloc. Les enfants acquièrent une connaissance des notions et des relations spatiales à travers de nombreuses situations mais, pour Reifel, le matériel bloc fournit des 
situations privilégiées pour expérimenter ces notions et témoigner de la progression de la compréhension des enfants en regard des notions et des relations spatiales.

Le tableau 1 présente les différents stades d'évolution dans l'activité de construction selon les divers auteurs qui ont travaillé à l'identification de ces stades (voir appendice A.2).

Les études de Johnson (1974) et des autres auteurs ayant contribué à décrire des stades d'évolution de la construction nous informent certes sur les différentes étapes et sur les caractéristiques de chacun des stades; cependant, même si tous ces auteurs identifient le stade du jeu dramatique vers cinq ans, aucun n'apporte d'éléments d'observation, de description et d'analyse sur la spécificité du jeu dramatique élaboré à partir du matériel bloc. Peut-il se comparer au jeu symbolique tel que décrit par Piaget? Existe-t-il des spécificités du jeu dramatique reliées directement à la nature même du médium utilisé, en l'occurrence les blocs? Ces constatations orientent les préoccupations abordées par la présente recherche.

\section{L'approche pédagogique}

Cette approche regroupe les recherches à caractère développemental où les blocs ont été réinvestis dans différentes didactiques disciplinaires. Ainsi, la didactique des mathématiques et des sciences mettent en évidence comment le matériel bloc contribue à l'acquisition et à la consolidation de diverses notions et concepts reliés à une discipline donnée et ce, à des fins pédagogiques.

Dans la plupart des études, les concepts mathématiques et scientifiques identifiés par les auteurs comme source de potentialités du matériel bloc sont analysés selon la théorie piagétienne. Sur le plan du développement intellectuel, les blocs permettent d'acquérir des habiletés en mathématiques et en sciences. Ils fournissent des repères intéressants dans l'apprentissage de différents concepts relatifs à la classification, aux relations de grandeur, de forme, de hauteur, de largeur, à l'aire, à la fraction et à des indices topologiques, d'intérieur, d'extérieur, d'ouverture, de fermeture, de près, de loin, à des notions d'espace et enfin, à l'identification des différents attributs de ce matériel. 
Les travaux de Leeb-Lundberg $(1970,1984)$ présentent un éventail exhaustif de l'apport du matériel bloc dans le développement des concepts mathématiques. Elle expose comment, à travers les diverses expériences de construction, l'enfant développe et perfectionne les concepts mathématiques et de la géométrie projective, euclidienne et topologique. Dans un cadre de référence piagétien, elle analyse les concepts de la conservation du nombre, de la longueur, des quantités, de la perspective, de la correspondance terme à terme, de la sériation et de la classification. De plus, elle met en évidence quelques implications pédagogiques et quelques suggestions d'interventions.

Enfin, des éléments explicatifs du matériel bloc de Pratt sont présentés et démontrent comment ce matériel a été conçu eu égard des différentes caractéristiques mathématiques en vue de l'acquisition et de la consolidation des notions de propriétés des blocs et des notions de relations. Ainsi, l'auteure établit un parallèle constant entre le développement cognitif et l'apprentissage des concepts mathématiques par l'intermédiaire des blocs.

Dans le même sens, diverses activités ont été élaborées à partir des blocs dans un but pédagogique afin de faire connaître et comprendre à l'enfant différents concepts reliés aux mathématiques (Beattie, 1986; Bruni and Silverman, 1977; Cartwirght, 1988 et Liedtke, 1975).

Pour sa part, Moffitt (1984) présente les blocs de construction comme un matériel qui permet à l'enfant de développer différents concepts de science tels que: le poids, la hauteur, la largeur, l'épaisseur, l'équilibre, la stabilité et le centre de gravité. Elle met en évidence la façon dont l'enfant, à travers ses expériences avec ce matériel, découvre ces différents concepts.

Quand l'enfant construit une structure ou représente le monde symboliquement avec des maisons, des ponts, des rampes et des tunnels, il négocie autant avec des concepts géographiques qu'avec des concepts scientifiques d'espace, de distance, de direction, de terrain (réseau), de modèles et de plans. Ce matériel est un médium particulièrement bien adapté pour permettre à l'enfant de réaliser les différentes étapes d'observation, de comparaison, de classification, de prédiction et d'interprétation utilisées dans la démarche scientifique traditionnelle. Il permet également l'apprentissage des différentes propriétés du matériel en fonction de la taille, de la forme, du poids, de sa qualité tridimensionnelle 
d'épaisseur, de largeur et de longueur. Les blocs représentent également un système et les différentes interactions internes d'un système. Les enfants apprennent différentes séquences de modèles et l'importance de la place qu'occupe chacun des blocs dans la construction ainsi que l'interaction de ces blocs entre eux.

\section{La recherche sur les blocs selon une perspective sociale}

La perspective sociale, considérée dans les recherches ayant comme objet d'étude le matériel bloc, regroupe les travaux où domine une conception qui met l'emphase sur certains déterminismes culturels et sociaux véhiculés par ce matériel et qui orientent l'activité de l'enfant.

Deux types de recherche se regroupent sous cette perspective: celles sur le matériel bloc et le développement social comme tel et celles sur le matériel bloc et les stéréotypes sexistes.

Une première voie de réflexion s'oriente vers le développement social de l'enfant et sur les éléments constitutifs mis en relation avec l'activité de l'enfant et les blocs. Brody (1984), dans un article intitulé "Social Studies and Self-awareness", trace les premiers jalons du potentiel des blocs liés au développement social de l'enfant. Par diverses expériences ayant comme médium les blocs, l'enfant développe sa capacité de construire des relations avec les adultes et avec les autres enfants et ce, à travers le temps et l'espace. L'enfant à cinq ans découvre ses compétences et ses habiletés face aux blocs et son groupe de pairs valorise ces nouvelles capacités. Les constructions de l'enfant se réalisent progressivement en coopération et il tente de recréer son environnement familier tel que sa rue, sa ville, des maisons, des magasins et des édifices. À cet âge, l'apparition des constructions de ville avec les gens qui travaillent à différents métiers et professions est de plus en plus fréquente. Ses constructions deviennnent plus complexes et demandent plus de temps pour leurs réalisations et, par le fait même, le jeu symbolique tributaire de ces constructions se manifeste souvent à la fin de la période de jeu, au moment même où l'enfant se dit prêt à jouer puisque sa construction est terminée.

Dans le même ordre d'idées, Rudolph et Cohen, déjà en 1964, soulevaient l'importance de ce matériel de jeu dans les programmes pour les jeunes enfants et soulignaient 
entre autres, comment les blocs peuvent promouvoir les relations sociales et la coopération. Après que l'enfant eût acquis des compétences et développé certaines habiletés dans un jeu solitaire avec ce matériel, il manifeste un intérêt pour investir dans une activité de construction avec d'autres enfants. En effet, à travers des expériences de construction en groupe, les enfants apprennent à s'organiser entre eux pour mener à terme une construction partagée.

À partir des éléments théoriques de réflexion proposés par Rudolph et Cohen, la relation entre le développement social et les blocs est devenue objet d'étude pour les chercheurs intéressés à la dimension sociale du développement avec le matériel bloc. Dans ce contexte, l'étude de Rogers (1985), "Relationships Between Block Play and the Social Development of Young Children", fait ressortir une problématique concernant les comportements sociaux et les niveaux de participation sociale des enfants d'âge préscolaire dans leur activité avec ce matériel. Cet auteur s'est également intéressé à la différence due au sexe qui apparaît dans l'utilisation de ce matériel.

Les vingt enfants qui ont participé à l'étude ont eu l'occasion de jouer avec deux types de blocs, ceux de Pratt et des gros blocs évidés. Les observations des comportements sociaux et des niveaux de participation sociale ont été codées sur une liste à cocher, élaborée par les chercheurs. En ce qui concerne la variable comportement social, les comportements ont été regroupés sous trois catégories distinctes: le comportement verbal, l'action et l'expression faciale/la vocalisation. Ces catégories ont été élaborées à partir des données théoriques et méthodologiques sur les comportements sociaux des enfants d'âge préscolaire observés par plusieurs chercheurs (Appleton, 1980; Blurton Jones, 1967, 1972; Leach, 1972; McGrew, 1972; Parten, 1932; Smith and Connoly, 1972: voir Rogers, 1985).

L'observation de la variable niveau de participation sociale s'est effectuée à partir de la grille de Rogers qui présente six niveaux: solitaire, parallèle, en groupe, non occupé, à l'intérieur de la construction et à l'extérieur de l'aire. Ces niveaux sont adaptés des travaux de Parten (1933) et utilisés par Smith and Connoly (1972). Les données ont été soumises à une analyse statistique (ANOVA). En résumé, les résultats montrent que les enfants manifestent des comportements sociaux significatifs sous la catégorie Comportement Verbal se traduisant par les énoncés: Accepte/Admet/S'excuse/ - Demande/ 
Offre/Suggère - Blâme/se plaint/Condamne et Refuse/Dément/Nie et ce, dans la situation où les enfants jouaient avec les gros blocs évidés.

De plus, ces chercheurs ont également observé que les enfants s'engageaient de façon significative dans des jeux de groupe, jeux coopératif et associatif, lorsqu'ils jouaient avec les gros blocs évidés et qu'ils s'engageaient à des niveaux plus solitaire et parallèle avec les blocs de Pratt.

Toujours dans une perspective sociale, une deuxième voie de réflexion oriente les chercheurs vers une préoccupation sur les stéréotypes sexistes. Ces études sont envisagées sous une dimension sociale puisque l'objet de recherche met en évidence les jeux et/ou le matériel de jeu traditionnellement considérés féminins ou masculins. Ces questionnements sur les stéréotypes sexistes font resurgir toute la problématique de l'influence culturelle et sociale par rapport à l'imprégnation ou le conditionnement social dans la différenciation des rôles relatifs au sexe dans notre société, en fonction des objets et des activités de jeu des enfants. Ces études apportent également des éléments de questionnement en fonction de l'objet ludique, le jouet, ici les blocs, comme médiateur, support et véhicule de la transmission des valeurs culturelles et sociales, tant par les moyens qu'il donne pour accéder à certaines formes de jeux culturellement déterminés, que par son potentiel intrinsèque, porteur de significations culturelles et sociales (Brougère, 1989). Dans ce sens, les études sur les stéréotypes sexistes en relation avec les jouets, les jeux et les activités de jeu mettent en parallèle quatre paramètres importants: les objets de jeu, le type de jeu, les manières de jouer et le temps investi dans le jeu.

Dans la plupart des sociétés, les différences dans le jeu entre garçons et filles ne sont pas seulement attendues mais activement encouragées (Milar, 1968). Encore aujourd'hui, ces différences sont vite reconnues, d'abord par les adultes, concepteurs de jouets, parents, éducateurs et consommateurs et ensuite, par les enfants eux-mêmes qui ont rapidement saisi les nuances subtiles qui existent entre les jouets proposés et leur rapport étroit avec les différents rôles qu'on attend d'eux. Les modes d'expression de jeu, les règles et les objets de jeu sont donc incontestablement aussi les produits d'une culture précise (Gianini, 1974). 
D'une façon générale, l'intérêt pour la différence des sexes dans le choix des activités de jeu ou matériel de jeu a fait depuis longtemps l'objet de nombreuses recherches. Déjà à la fin du XIXe siècle et au début du XXe siècle, certains auteurs (Crosswell, 1896; McGhee, 1898; Terman, 1921: voir Beeson and Williams, 1979) reconnaissaient dans leurs études empiriques que les filles jouaient à des jeux traditionnellement considérés féminins et que les garçons jouaient à des jeux traditionnellement considérés masculins. Ces études ont mis l'emphase seulement sur les préférences de jeu des garçons et des filles âgés de six à 18 ans et ont mis en lumière l'existence d'une plus grande variété d'activités de jeux pour les garçons en comparaison avec les filles.

D'autres études, dont l'intérêt porte sur le choix des enfants face aux objets de jeu et aux activités chez les jeunes d'âge préscolaire, démontrent bien la grande popularité des blocs comme choix d'activités, comme investissement et intérêt soutenu des enfants sur de longues périodes de temps et comme contribution des interactions sociales entre les enfants (Hartley, Frank et Goldenson, 1952; Hulson et Reich, 1931; Margolin et Leton, 1961; Toshiko, Tomoko et Takahashi, 1974; Van Alystyne, 1932 et Vlietstra, 1978).

Les blocs sont souvent considérés comme étant des objets à caractère neutre, c'està-dire adaptés aux enfants des deux sexes. Cependant, malgré cette caractéristique, ils sont souvent investis d'une empreinte beaucoup plus masculine que féminine. À cet effet, l'aire des blocs est souvent établie comme un territoire de garçons dans les classes maternelles. Dans ce sens, le matériel bloc est également reconnu comme un objet de jeu et une activité de garçons. Les filles se sont souvent vues exclues, physiquement et verbalement, de cette chasse-gardée des garçons.

Des recherches ont donc été réalisées afin d'investiguer s'il existe une préférence de sexe liée à l'aire des blocs (Sprung, 1975, 1978). Les résultats de ces différentes études ne font pas consensus. Certaines révèlent que les garçons choisissent beaucoup plus cette activité (cinq fois plus) que les filles et jouent plus longtemps (deux fois plus) (Cramer et Hogan, 1975; Fagot, 1974; Farrell, 1957; Robinson, 1977; Rubin, 1977 et Smith, 1977), tandis que d'autres études nuancent ces résultats en observant qu'à opportunité égale de jouer avec ce matériel, la complexité des structures, le temps accordé à la construction, le nombre de blocs et d'accessoires utilisés ne démontrent aucune 
différence significative entre les garçons et les filles (Beeson et Williams, 1979-1980; Kinsman et Berk, 1979; Massey, 1969 et Varma, 1980).

\subsection{L'énoncé du problème et les questions générales de recherche}

La pré-expérimentation de cette recherche a démontré que les enseignantes n'ont pas toujours un intérêt soutenu pour rendre aux enfants le matériel bloc accessible en tout temps. Cette attitude semble surtout due en grande partie à des connaissances fragmentaires sur les potentialités des blocs, des différentes voies possibles d'exploitation et de la contribution spécifique qu'il peut apporter au développement cognitif et social de l'enfant.

Les quatre dimensions étudiées ont permis d'envisager le matériel bloc dans sa complexité et fournissent des indicateurs essentiels à notre problématique de recherche et permettent d'interroger la présence, la pertinence, la place, l'accessibilité, la connaissance et l'exploitation de ce matériel dans les classes maternelles. Pour chacune de ces dimensions analysées, rappelons les indicateurs essentiels qui conduisent à la formulation de la question générale de recherche de cette étude.

Vu comme objet industriel, c'est une prise en compte d'un matériel qui s'est revêtu d'allures particulières au gré des transformations de chaque époque et qui demeure un objet ludique questionnant face à l'intérêt manifesté par les enfants. Il est encore un objet contemporain qui mérite un regard réflexif sur ses potentialités et sur les activités qu'il suscite.

Considéré comme objet de jeu, le matériel bloc se définit par son caractère permanent, puisqu'il demeure un objet de jeu en dehors du contexte du jeu. Il possède un potentiel qui s'actualise par les multiples fantaisies de l'enfant.

Envisagé sous un point de vue psychologique, ce sont les potentialités développementales de l'objet par rapport aux différentes facettes de la personnalité de l'enfant qui sont interrogées. Dans cette étude, nous questionnons plus spécifiquement le potentiel du matériel bloc comme outil et support à l'activité de construction et à l'activité de jeu symbolique de l'enfant de cinq ans. 
Envisagé sous un point de vue social, ce sont les blocs comme objet médiateur dans notre société occidentale qui fournissent différents éléments de réflexion. Par l'intermédiaire d'un matériel donné, en l'occurrence les blocs, l'adulte, concepteur, fabricant et consommateur, inscrit des messages culturels et sociaux dans la matérialité même de l'objet et surtout dans sa représentation. Cette représentation ou dimension symbolique s'actualise à deux niveaux. Le premier niveau, où l'objet représente quelque chose du réel ou de l'imaginaire de l'enfant, est imprégné des visions et des attentes de l'adulte quant à l'orientation du jeu de l'enfant. Le deuxième niveau invite à des jeux symboliques, d'où l'aspect dynamique de ce niveau puisque l'enfant entre en interaction avec l'objet. Ici, les significations culturelles et sociales de l'objet obligent soit l'enfant à jouer en conformité avec ces significations ou bien au contraire, ces mêmes significations entraînent l'enfant dans un jeu de confrontation, d'ajustement et d'appropriation des contenus culturel et social de l'objet afin qu'il construise lui-même un sens nouveau et des significations nouvelles qualifiées d'individuelles et de personnalisées. Ainsi, ce questionnement par rapport au bloc vu comme objet de jeu, interroge sur le message inscrit dans la matérialité même des blocs et de ses représentations et sur l'aspect dynamique de ce matériel quand il se retrouve entre les mains des enfants et que ceux-ci s'aventurent soit à la reproduction des significations culturelles et sociales, soit à la construction individuelle de ces significations.

$\mathrm{Vu}$ comme objet éducatif et pédagogique, c'est une analyse des philosophies et des méthodes d'éducation du jeune enfant qui ont eu cours depuis le XIX ${ }^{\mathrm{e}}$ siècle, associée conjointement aux différentes conceptions du jeu et du jouet depuis la fin de la Renaissance où, époque après époque, ces derniers se sont vus qualifiés d'éducatifs, de pédagogiques et de développementaux, qui a mis en évidence le matériel bloc comme élément important, voire même essentiel, dans l'élaboration de modèle d'éducation et dans la création de programmes pour les enfants d'âge préscolaire. Cette lecture a permis de démontrer l'intérêt, l'importance et la place privilégiée des blocs comme matériel, autant dans les réflexions que dans l'opérationnalisation des principes à la base des méthodes d'éducation préscolaire, que dans l'élaboration des programmes. C'est là une reconnaissance d'un fort potentiel du matériel bloc comme élément moteur de nombreuses sources d'exploitation. 
Enfin, vu comme objet de recherche, l'analyse des différentes études réalisées à partir du matériel bloc a permis de recadrer l'ensemble de ces travaux selon deux perspectives: la perspective psychologique et la perspective sociale.

Dans une perspective psychologique, c'est la dimension développementale qui retient davantage notre attention par rapport à notre préoccupation de recherche. À partir des travaux de Johnson (1974) qui a identifié six stades d'évolution de la construction, d'autres auteurs ont, à travers leur recherche, raffiné et complété ces stades. Cependant, même si tous ont observé le stade du jeu dramatique vers cinq ans, aucun d'entre eux n'apporte d'éléments d'observation, de description et d'analyse sur la spécificité de ce jeu dramatique élaboré à partir du matériel bloc.

Dans une perspective sociale, les études sur le développement social de l'enfant et sur ces éléments constitutifs mis en relation avec l'activité de l'enfant et les blocs suscitent nombre de questionnements quant au caractère social du jeu symbolique réalisé avec le matériel bloc. Vers cinq ans, étant donné le développement de leurs compétences cognitives et sociales, les enfants abandonnent peu à peu un jeu plus solitaire et parallèle pour s'adonner à des jeux à deux, à trois et à quatre. Les différents éléments théoriques de ces recherches apportent donc des aspects de réflexion intéressants par rapport à la problématique de notre étude puisqu'ils mettent en évidence les concepts de coopération, de communication d'idées, d'acceptation des idées des autres, de négociations avec l'environnement, avec les pairs et avec les adultes, donc d'interactions sociales. La prise en compte de ces concepts est intéressante à réinvestir sur les plans théorique et méthodologique de la présente recherche.

Dans la même perspective sociale, les études sur les stéréotypes sexistes remettent en surface les questionnements relatifs à l'influence culturelle et sociale par rapport à l'imprégnation culturelle et le conditionnement social dans la différenciation des rôles masculins et féminins dans notre société, en fonction des objets et des activités de jeu des enfants. Ces études situent donc les blocs comme étant un objet à caractère neutre mais investi toutefois d'une empreinte beaucoup plus masculine que féminine. On établit souvent cet espace de jeu comme un territoire de garçons et on reconnaît communément cet objet de jeu et cette activité comme une chasse-gardée des garçons. Ainsi, si les garçons 
et les filles ont les mêmes possibilités de fréquentation et de temps accordé à cette activité, que peut-on observer des productions, du temps investi, des scénarios élaborés, etc.?

Considérant les différents éléments dégagés de chacune des quatre perspectives du matériel bloc:

quant aux potentialités particulières de ce matériel comme outil et support à l'activité de construction et à l'activité de jeu symbolique de l'enfant de cinq ans;

quant aux dimensions symboliques s'actualisant à un premier niveau dans l'activité de construction même et à un deuxième niveau dans l'activité de jeu symbolique simultanée ou subordonnée à l'activité de construction;

quant à sa dimension développementale qui décrit les stades d'évolution de l'activité de construction, identifiant le stade du jeu dramatique à cinq ans, sans toutefois n'apporter aucun élément d'observation, de description et d'analyse sur la spécificité du jeu dramatique élaboré à partir du matériel bloc;

quant aux divers questionnements reliés au caractère social de l'activité de construction à cet âge et de l'activité du jeu symbolique réalisé avec le matériel bloc;

deux questions générales de recherche émergent:

Quelle est la nature des activités que le matériel bloc suscite chez I'enfant?

Est-ce que ce matériel et ces activités contribuent au développement cognitif et social de l'enfant qui fréquente la classe maternelle?

\subsection{Récapitulation-synthèse}

Notre examen des quatre dimensions du matériel bloc sous différents regards tels que: objet industriel, objet de jeu, objets éducatif et pédagogique et objet de recherche, a conduit à soulever un certain nombre de questions et à identifier certains éléments théoriques relatifs à alimenter la problématique de cette étude. 
Afin de bien faire ressortir la trame de fond de ce questionnement, un tableausynthèse a été réalisé. Ce tableau présente donc les quatre dimensions analysées et les questions et/ou éléments théoriques s'y rapportant. 
Tableau 1.1

\section{RÉCAPITULATION-SYNTHÈSE}

\begin{tabular}{|c|c|c|c|c|}
\hline Introduction & $\begin{array}{l}\text { Le matériel bloc vu } \\
\text { comme objet } \\
\text { industriel }\end{array}$ & $\begin{array}{c}\text { Le matériel bloc vu comme } \\
\text { objet de jeu }\end{array}$ & $\begin{array}{l}\text { Le matériel bloc vu } \\
\text { comme objet éducatif } \\
\text { et pédagogique }\end{array}$ & $\begin{array}{l}\text { Le matériel bloc vu comme } \\
\text { objet de recherche }\end{array}$ \\
\hline $\begin{array}{l}\text { Mode d'appréhension } \\
\text { du monde de l'enfant de } \\
5-6 \text { ans } \rightarrow \text { mode sym- } \\
\text { bolique. } \\
\text { Possibilités symboli- } \\
\text { ques pouvant se mani- } \\
\text { fester dans le coin bloc. } \\
\text { Lieu - occasion d'expé- } \\
\text { rimenter tout un uni- } \\
\text { vers avec des blocs de } \\
\text { bois vernis. } \\
\text { Jeu peut s'exprimer } \\
\text { d'une manière tout à } \\
\text { fait originale dû en } \\
\text { grande partie au poten- } \\
\text { tiel même du matériel } \\
\text { que sont les blocs et } \\
\text { des activités qu'ils } \\
\text { suscitent chez l'enfant. } \\
\text { Identification de la con- } \\
\text { tribution du matériel } \\
\text { bloc au développement } \\
\text { de l'enfant de 5-6 ans, à } \\
\text { une période fructueuse } \\
\text { sur le plan symbolique. } \\
\text { Rôle des interactions } \\
\text { sociales dans cette dy- } \\
\text { namique de jeu. }\end{array}$ & $\begin{array}{l}\text { - Objet de jeu toujours } \\
\text { présent. } \\
\text { - Objet-jouet question- } \\
\text { nant face à l'intérêt qu'il } \\
\text { a suscité et qu'il conti- } \\
\text { nue de susciter autant } \\
\text { chez les petits que chez } \\
\text { les grands. } \\
\text { - Maintient l'enthousias- } \\
\text { me autant de la part des } \\
\text { fabricants que des } \\
\text { enfants. } \\
\text { - Dans la classe maternel- } \\
\text { le, a une place et capti- } \\
\text { ve les enfants durant de } \\
\text { longues heures lorsque } \\
\text { l'enseignante leur en } \\
\text { donne l'occasion. } \\
\text { - Bloc de Caroline Pratt } \\
\text { - grande popularité. } \\
\text { - Pas dans les salles de } \\
\text { jeux des enfants à la } \\
\text { maison. } \\
\text { Donc matériel à décou- } \\
\text { vrir à la maternelle, tant } \\
\text { au niveau de sa matéria- } \\
\text { lité que des possibilités } \\
\text { d'expériences de jeu. } \\
\text { - Objet à la fois ancien et } \\
\text { contemporain qui méri- } \\
\text { te un regard réflexif sur } \\
\text { ses potentialités et sur } \\
\text { les activités qu'ils sus- } \\
\text { citent. }\end{array}$ & $\begin{array}{l}\text { Deux sens au terme jouet: } \\
\text { - caractère éphémère; } \\
\text { - caractère permanent lié à sa matérialité } \\
\text {-> bloc - potentiel latent intrinsèque } \\
\text { qui se traduit à travers l'activité de } \\
\text { l'enfant. } \\
\text { Point de vue psychologique: } \\
\text { Les qualités du jouet sont directement } \\
\text { reliées aux différentes valeurs issues du } \\
\text { caractère développemental de l'objet du } \\
\text { jeu. } \\
\text { Valeur de relation: comment le jouet va- } \\
\text { t-il mettre l'enfant en rapport avec les } \\
\text { autres et avec son environnement (Cam- } \\
\text { pagne, 1989). } \\
\text { Valeur relationnelle: elle donne du pou- } \\
\text { voir au jouet en servant d'agent de liai- } \\
\text { son entre l'enfant et les autres enfants } \\
\text { ou l'adulte (DeGrandmont, 1989). } \\
\text { Point de vue social: } \\
\text { Fournit des indices quant à une possible } \\
\text { empreinte sociale que porte déjà l'objet } \\
\text { en lui - dans sa dimension matérielle et } \\
\text { dans sa dimension symbolique. } \\
\text { Dimension matérielle: } \\
\text { Les blocs de Caroline Pratt -> Plusieurs } \\
\text { formes variées => activité de construc- } \\
\text { tion. } \\
\text { Orientent le genre de production: } \\
\text { - différents genres d'édifices (maison, } \\
\text { château, garage, hôpital); }\end{array}$ & $\begin{array}{l}\text { Apprécier l'intérêt, l'impor- } \\
\text { tance et la place privilégiée } \\
\text { des blocs comme matériel } \\
\text { qui a servi: } \\
\text { - à alimenter des réflexions } \\
\text { sur l'éducation; } \\
\text { - à concrétiser des grands } \\
\text { principes à la base des dif- } \\
\text { férentes philosophies et/ } \\
\text { ou méthodes; } \\
\text { - à élaborer des programmes } \\
\text { d'éducation préscolaire. } \\
\text { Demeure aujourd'hui un ma- } \\
\text { tériel riche en potentiel } \\
\text { offrant plusieurs sources } \\
\text { d'exploitation, moteur mê- } \\
\text { me de notre questionnement. }\end{array}$ & $\begin{array}{l}\text { Perspectives psychologi- } \\
\text { ques: } \\
\text { Approche développementale: } \\
\text { - stades ou étapes d'évolution spé- } \\
\text { cifiques à l'activité de cons- } \\
\text { truction. } \\
\text { Stade 6: Apparition d'une forte } \\
\text { impulsion au jeu dramatique } \\
\text { autour et à partir des construc- } \\
\text { tions de blocs; } \\
\text { - jeu dramatique devient coopératif } \\
\text { et interrelié entre les enfants. } \\
\\
\text { Questionnement: } \\
\text { Différentes études de Johnson et } \\
\text { des autres auteurs ayant contribué } \\
\text { à décrire des stades d'évolution de } \\
\text { la construction nous informent sur } \\
\text { les différentes étapes et caractéristi- } \\
\text { ques de chacune des étapes. } \\
\text { Même si tous ces auteurs identi- } \\
\text { fient le stade du jeu dramatique } \\
\text { vers 5-6 ans, aucun n'apporte d'é- } \\
\text { léments d'observation, de descrip- } \\
\text { tion et d'analyse sur la spécificité } \\
\text { du jeu dramatique élaboré à partir } \\
\text { du matériel bloc. } \\
\text { Peut-il se comparer au jeu symbo- } \\
\text { lique tel que décrit par Piaget? } \\
\text { Existe-t-il des spécificités du jeu } \\
\text { dramatique reliées directement à la } \\
\text { nature même du médium utilisé, } \\
\text { en l'occurrence les blocs? }\end{array}$ \\
\hline
\end{tabular}




\begin{tabular}{|c|c|c|c|c|}
\hline Introduction & $\begin{array}{c}\text { Le matériel bloc vu } \\
\text { comme objet } \\
\text { industriel }\end{array}$ & $\begin{array}{c}\text { Le matériel bloc vu comme objet } \\
\text { de jeu }\end{array}$ & $\begin{array}{c}\text { Le matériel bloc vu } \\
\text { comme objet éducatif } \\
\text { et pédagogique }\end{array}$ & $\begin{array}{l}\text { Le matériel bloc vu comme } \\
\text { objet de recherche }\end{array}$ \\
\hline $\begin{array}{l}\text { Étude de l'activité de } \\
\text { l'enfant suscitée par le } \\
\text { matériel bloc -> la pro- } \\
\text { blématique de cette re- } \\
\text { cherche: } \\
\text { - questionnement maté- } \\
\text { riel et environnemen- } \\
\text { tal } \\
\text { - questionnement struc- } \\
\text { tural et interactionnel }\end{array}$ & & 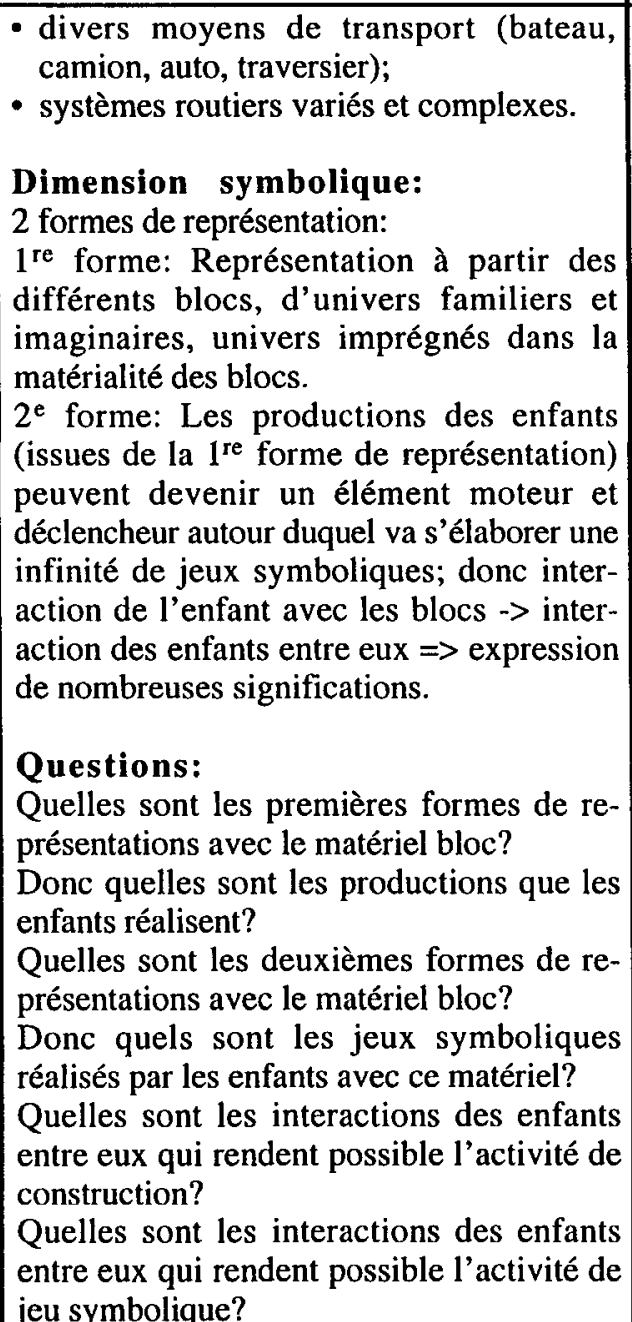 & & $\begin{array}{l}\text { Perspective sociale: } \\
\text { Développement social de l'enfant } \\
\text { et sur les éléments constitutifs. Ja- } \\
\text { lons potentiels des blocs en rap- } \\
\text { port avec le développement social } \\
\text { de l'enfant. } \\
\text { - par diverses expériences ayant } \\
\text { comme médium les blocs, l'en- } \\
\text { fant développe sa capacité de } \\
\text { construire des relations avec les } \\
\text { adultes et les autres enfants et } \\
\text { ce, à travers le temps et l'espa- } \\
\text { ce; } \\
\text { - les constructions des enfants se } \\
\text { réalisent progressivement en } \\
\text { coopération; } \\
\text { - Rudolph et Cohen (1964) souli- } \\
\text { gnent comment les blocs peu- } \\
\text { vent promouvoir les relations } \\
\text { sociales et la coopération; } \\
\text { - intérêt manifesté pour investir } \\
\text { dans une activité de construction } \\
\text { avec d'autres enfants, après l'ac- } \\
\text { quisition de compétences et } \\
\text { d'habiletés; } \\
\text { - à travers des expériences de cons- } \\
\text { truction en groupe, les enfants } \\
\text { apprennent à s'organiser entre } \\
\text { eux pour mener à terme une } \\
\text { construction partagée. }\end{array}$ \\
\hline
\end{tabular}




\section{CHAPITRE II}

\section{D'UN QUESTIONNEMENT INTUITIF DE L'ACTIVITÉ DE L'ENFANT AVEC LE MATÉRIEL BLOC À UN RECADRAGE ÉPISTÉMOLOGIQUE ET CONCEPTUEL DE L'OBJET DE RECHERCHE}

La réponse aux questions de recherche telles que formulées à la fin du premier chapitre oblige à une réflexion épistémologique et conceptuelle sur la nature de l'activité de l'enfant avec le matériel bloc. Cette réflexion est alimentée par deux sources d'éléments théoriques reliées à cette activité.

Une première source s'oriente vers les différents travaux sur le jeu symbolique. Ayant identifié au début de la problématique de cette recherche, que l'enfant de cinq ans, fréquentant la classe maternelle, se situe sur le plan de son développement cognitif dans une période où il appréhende le monde qui l'entoure sous un mode symbolique lequel se manifeste dans son activité ludique, nous supposons que l'activité suscitée par le matériel bloc est également teintée de ce mode symbolique d'appréhension du monde. De plus, l'ensemble des auteurs qui ont travaillé à l'élaboration des différents stades d'évolution de la construction ont identifié un stade caractérisé par une forte impulsion au jeu dramatique autour et à partir des productions des enfants. Cependant, aucun de ces auteurs n'apporte d'éléments spécifiques de ce jeu dramatique élaboré à partir du matériel bloc. En conséquence, le présent chapitre examine la pensée de Piaget et de Vygotsky sur le développement de la connaissance, de l'enfant et du jeu et plus particulièrement du jeu symbolique, puisque ces deux théoriciens orientent et alimentent l'ensemble des travaux de recherche sur le jeu symbolique. Par la suite, sont présentés les postulats des chercheurs qui ont complété et raffiné les travaux de ces pionniers. 
Une deuxième source d'éléments théoriques nous entraîne à examiner l'élément social dans l'activité de l'enfant avec le matériel bloc dans le contexte de la classe maternelle, étant donné l'âge des enfants et la valeur de relation que l'on attribue à l'objet de jeu, ici, les blocs, comme agent de liaison et d'interaction entre les enfants. Suite à ce questionnement de l'élément social dans la dynamique de l'activité de l'enfant avec le matériel bloc, le concept d'interaction sociale apparait comme un élément important voire même peut-être structurant de l'activité de construction et de l'activité de jeu symbolique générées par ce matériel. Nous considérons donc ce concept d'interaction sociale à la lumière des conceptions du développement de l'intelligence de Piaget (1964), de Vygotsky (1966) de Doise et Mugny (1981) et de Perret-Clermont (1979). Par la suite, des travaux de recherche sur les interactions sociales et le jeu symbolique social seront investigués puisqu'à cinq ans, le jeu dramatique deviendrait coopératif et interrelié entre les enfants. Selon les données de certaines études sur le développement social et de ses éléments constitutifs, les blocs semblent être un matériel suscitant diverses expériences où l'enfant peut développer des compétences relationnelles avec les autres enfants. Les constructions des enfants se réaliseraient progressivement en coopération et les blocs semblent promouvoir les relations sociales et la coopération (Brody, 1984 et Rudolph et Cohen, 1964).

De ces deux sources d'éléments théoriques émerge donc un cadre conceptuel pour comprendre la dynamique de l'activité de l'enfant avec le matériel bloc. Le problème de cette étude, rappelons-le, implique deux pôles de questionnement. Le premier concerne les potentialités du matériel bloc et le contexte environnemental dans lequel s'initie, se déroule et se termine cette activité. Le deuxième pôle, plus structural et interactionnel, interroge la nature et la structure de l'activité de l'enfant suscitée par ce matériel particulier dans le contexte spécifique de la classe matemelle.

\subsection{Vers une compréhension du jeu et du jeu symbolique}

Différentes définitions et nombreuses fonctions ont été attribuées au jeu et ce, dans des cadres de références où émergent des visions particulières de l'activité ludique. De plus, le jeu a fait l'objet de diverses classifications en fonction de critères de regroupement plus ou moins bien définis et a entraîné plusieurs types de classement selon des perspectives philosophiques, psychologiques, didactiques et pédagogiques, où chacun visait à appuyer sa conception personnelle du jeu. 
L'intérêt pour le jeu n'est plus à contester parmi les chercheurs, car rares sont ceux qui vont s'objecter à son apport important sur le plan du développement de la personnalité de l'enfant. C'est, à notre avis, le point le plus convergent dans l'ensemble de la recension des écrits sur le sujet. Il existe également des divergences et c'est spécifiquement sur ces deux éléments, de convergence et de divergence, qu'il convient de réfléchir pour une analyse plus approfondie.

Pour comprendre les éléments de convergence et de divergence en matière de définitions, de fonctions et de classification du jeu, il est primordial de les situer à l'intérieur d'un cadre de référence. Pour ce faire, il faut réaliser un regroupement de ces visions ou conceptions des concepts de jeu et de jeu symbolique à partir de notre propre position de recherche et de nos choix épistémologiques, quant à notre façon de concevoir la connaissance, le jeu, le jeu symbolique et l'environnement de la classe maternelle.

Dans une démarche de recherche, tenter de faire des liens entre différentes théories ou tenter de comprendre les divergences des conceptions qui s'offrent à nous, relève du véritable défi. Dans notre cas, c'est la réflexion épistémologique qui a permis d'éclairer et de trouver un fil conducteur pour mieux situer les approches, les courants, les théories et les modèles. Cette réflexion épistémologique est le terrain, le fil conducteur, la toile de fond et le filtre d'analyse autour duquel s'articule un questionnement par rapport à la démarche de notre recherche.

Pour relever ce défi, il fallait trouver un moyen de systématiser la pensée des différents auteurs qui ont contribué à alimenter et à documenter le domaine du jeu symbolique.

Quatre temps forts ont marqué la recherche sur le jeu symbolique. Un premier temps fort se situe vers les années 20 et les débuts de 1930 et Buehler, Stern, Palmer, Griffiths, Markey, Robinson et Valentine sont des chercheurs faisant figure dominante pendant cette période. Malgré bon nombre de théoriciens préoccupés par le jeu symbolique, peu de recherches empiriques ont vu le jour à cette époque.

Un deuxième temps fort entre les années 1940 et 1950 témoigne de la perspective psychanalytique qui a donné l'essor à la recherche sur la thérapie par le jeu et sur le jeu des poupées. Sears (1947) semble l'investigateur d'une conception plus psychanalytique 
du jeu symbolique qui découle de la théorie freudienne. Même si Freud n'a pas articulé une théorie du jeu, il a inspiré, par la suite, plusieurs auteurs (Papalia et Olds, 1989; Rubin et al., 1984 et Singer, 1973) pour qui le jeu symbolique devenait un moyen d'expression des tensions émotives de l'enfant. Les premières recherches sur le jeu symbolique, dans un cadre théorique d'interprétation psychanalytique et de l'apprentissage social, avaient pour objet d'étude le contenu du jeu des enfants (Almy et al., 1982; Fein, 1981 et Rubin et al., 1984).

Un troisième temps fort, au début des années 1970, a fait en quelque sorte renaître le jeu symbolique au sein des préoccupations des chercheurs. Les études réalisées à cette époque se sont largement inspirées des travaux de Piaget (1962), Singer (1973) et SuttonSmith (1971). C'est à partir du milieu des années 70 qu'on assiste à une recrudescence de travaux de recherche sur le jeu symbolique. Cette période demeure particulièrement fructueuse sur le plan de la recherche sur le jeu symbolique, associée à une multitude de thématiques.

Pour nous, s'ajoute un quatrième temps fort de la recherche sur le jeu symbolique, soit le début et le milieu des années 80 , qui continue d'influencer et d'alimenter la recherche actuelle. Premièrement, au milieu des années 80 , des conceptions théoriques du développement de la connaissance centrées sur une dimension sociale beaucoup plus influente et structurante qu'on l'avait laissé supposer jusque là, s'infiltrent à travers les recherches sur le jeu symbolique. Deuxièmement, une reconsidération du point de vue de Vygotsky sur la conception du développement de la connaissance et du jeu symbolique refait surface dans les écrits scientifiques et oriente un bon nombre de recherches dans ce domaine. Troisièmement, des réflexions épistémologiques de la recherche en sciences humaines et sociales, quant aux modes de productions des connaissances, viennent interroger les méthodologies quantitatives et la recherche quantitative en général qui avaient, jusqu'à maintenant, été les seules envisagées autant dans la recherche en psychologie qu'en éducation. Donc l'avènement encore bien timide d'une recherche de type plus compréhensive commence à son tour à influencer la recherche sur le jeu symbolique. Notons que du côté européen, surtout en France, certains travaux sur le jeu symbolique sont abordés selon une conception plus qualitative de la recherche. 
En somme, la consultation des écrits sur le jeu symbolique fait prendre conscience qu'on ne peut faire autrement que d'accorder à la théorie de Piaget un statut bien particulier, puisqu'il n'a cessé d'être le pivot majeur d'un grand nombre d'études récentes sur le jeu (Vandenplas-Holper, 1987) et sur le jeu symbolique (Fein, 1981). Par la suite, la renaissance des idées de Vygotsky a entraîné un nouveau sillon de recherche sur le jeu symbolique.

Comme Piaget a soutenu théoriquement l'orientation de nombreuses études sur le jeu symbolique, ceux qui par la suite ont rejoint les rangs de théoriciens du jeu symbolique et qui sont le plus couramment cités aujourd'hui (Bateson, 1955; Bruner et al., 1976; Singer, 1973; Sutton-Smith, 1971 et Vygotsky, 1966) ont défini leur théorie et leur position à partir du pivot piagétien, soit d'une manière convergente à l'ensemble ou à quelques concepts piagétiens, soit d'une manière divergente à la position défendue par Piaget. C'est pourquoi, avant de poser les bases de nos présupposés théoriques pour cette étude de l'activité de l'enfant avec le matériel bloc, nous retraçons les grandes lignes des théories de Piaget et de Vygotsky.

\subsubsection{Piaget, une conception du développement de l'intelligence et des connaissances où l'aspect individuel est central}

La position épistémologique de Piaget se situe dans un paradigme constructiviste. Dans ce paradigme, c'est la prise en compte de l'interaction sujet et objet qui est centrale; c'est la prise en charge et la responsabilité de chaque sujet envers l'appropriation, l'organisation, la gestion et enfin, la construction de sa connaissance. Chaque personne possède un potentiel latent susceptible de s'actualiser selon la chimie des facteurs intrinsèques et extrinsèques. Le sujet devient donc l'artisan de sa connaissance.

La connaissance et son développement sont des éléments-clés de toute l'œuvre épistémologique de Piaget et l'orientation de ses travaux s'est sans cesse justifiée en tentant d'identifier et d'approfondir ce qu'est la connaissance et comment elle se développe. Cette perspective, qualifiée de constructiviste, considère le processus actif du sujet dans l'élaboration et la construction de sa propre pensée. Piaget met en évidence, par une épistémologie génétique, l'importance des processus internes dans la structure d'élaboration de la connaissance et ajoute, également, que cette élaboration n'est rendue possible et 
complète que si elle s'effectue en interaction avec les objets et les personnes de l'environnement.

À partir de cette interaction que Piaget considère comme fondamentale, la connaissance va s'orienter en deux directions mais complémentaires, conduisant à l'élaboration de deux types de connaissances: la connaissance physique (connaissance exogène) et la connaissance logico-mathématique (connaissance endogène). La connaissance physique s'élabore à partir des propriétés mêmes de l'objet et la connaissance logico-mathématique s'articule à partir des relations que l'enfant établit entre les différentes propriétés des objets et des personnes de son environnement. C'est en fait une prise de conscience par le sujet de ses propres actions sur les objets.

Dans cette perspective de la construction de la connaissance, Piaget a identifié cinq mécanismes, constamment à l'œuvre tout au long du développement, qui sont à la base de la régulation du développement ou de la connaissance. En effet, le développement se réalise grâce au processus d'assimilation, d'accommodation, d'adaptation, d'organisation et d'équilibration. Ces processus constituent le cœur et le moteur du développement puisqu'ils définissent l'interaction adaptative du sujet avec le milieu, source du développement de l'intelligence et des connaissances.

L'articulation de ces processus s'effectue par groupe de deux. L'assimilation et l'accommodation sont toujours en interdépendance puisque l'une est la résultante de l'action du sujet sur les choses pour se les approprier et les intégrer, et l'autre est la résultante de l'ajustement du sujet aux contraintes imposées par le milieu. Ces deux processus sont donc au centre des deux autres processus d'adaptation et d'organisation qui permettent au sujet de s'adapter au milieu et d'organiser l'ensemble de ses conduites en fonction de l'adaptation imposée par le milieu.

L'alternance de ces processus assimilation/accommodation et adaptation/organisation ne vise qu'un seul but, celui de la recherche d'un état d'équilibre constant entre le sujet et le milieu. Le sujet, pour assurer son développement, est sans cesse à la recherche de cet équilibre et c'est au processus d'équilibration que revient la tâche de réaliser une synthèse des quatre autres processus. 


\subsubsection{Piaget, une conception du jeu et du jeu symbolique à travers le filtre psycho-individuel où l'aspect cognitif est central}

Dans la construction de sa connaissance, l'individu est l'agent essentiel et la connaissance se construit d'une manière significative grâce aux différentes confrontations de l'individu issues, d'une part, de ses actions sur l'environnement et, d'autre part, de la signification et des liens de ces actions avec ce qu'il connaît déjà. La nature de ces confrontations demeure essentiellement individuelle. Piaget parle donc d'élaboration des mécanismes de l'intelligence intra-individuelle.

En effet, parallèlement au développement cognitif, il élabore une classification du jeu qui tient compte de l'aspect évolutif et hiérarchique de toutes les formes ludiques essentielles, des plus simples aux plus complexes. Les divers groupes ludiques et leurs catégories fondamentales sont définis selon deux critères, celui de la recherche d'une prédominance d'une action sur une autre et celui de l'ordre successif d'apparition de ces formes ludiques. L'exercice, le symbole et la règle recouvrent, d'après lui, l'essentiel du vécu ludique de l'enfance à l'adolescence. Un quatrième palier intermédiaire, la construction, est proposé comme moyen terme entre les trois autres.

Pour Piaget, le jeu est l'activité principale des enfants, c'est par le jeu qu'ils se développent. Pour le très jeune enfant, le jeu est un mode d'appréhension important du monde qui l'entoure. Il est difficile d'établir une frontière entre ce qui est jeu et non-jeu et, par conséquent, Piaget interroge les schèmes réflexes dans cette frontière. Cependant, il parle de jeu dès les deux premiers mois d'existence de l'enfant et la démarcation entre l'activité de connaissance et l'activité ludique se constituerait à partir de la mimique de l'enfant pendant son activité. Cette mimique devient donc un indicateur précieux dans le qualificatif ludique attribué à l'activité de l'enfant.

Selon la classification du jeu de Piaget, trois structures constituent la trame évolutive du jeu: l'exercice, le symbole et la règle. Dès les premiers mois, l'enfant, par tâtonnements, explorations, manipulations et déplacements successifs, répète toutes sortes de gestes et de mouvements sans intention précise. Peu à peu, au hasard de ses expériences et découvertes, il prend plaisir à cette répétition, au résultat immédiat et à la diversité des effets produits d'abord fortuitement. Son activité ludique à cette période 
porte donc essentiellement sur des événements et des objets réels pour le simple plaisir des résultats obtenus. Vers deux ans, les jeux symboliques apparaissent. Le symbole implique la représentation d'un objet par un autre. Ainsi, l'enfant se détache progressivement de l'action immédiate puisqu'il est capable de se représenter le réel par l'intermédiaire de signes et de symboles; il est capable d'évoquer des situations, des personnes ou des objets absents. Son activité ludique est caractérisée par l'utilisation de la simulation, la fiction, la représentation, l'invention et la reproduction de personnages et d'événements. Enfin vers quatre-sept ans, débutent progressivement les jeux de règles. La règle suppose nécessairement les relations sociales ou interindividuelles. La règle évoque donc une idée de conventions et d'obligations réciproques que chaque partenaire de jeu doit respecter pour parvenir à un possible de jeu. Ces règles sont d'abord (vers sept-11 ans) simples et concrètes, directement liées à l'action et souvent supportées par des objets ou des accessoires bien définis. Par la suite, (vers 12 ans), ces règles se complexifient et deviennent indépendantes de l'action en se situant, de plus en plus, sur un plan d'abstraction. Ainsi, l'adolescent établit son raisonnement à partir d'hypothèses, de déductions, de combinaisons et de stratégies intériorisées. Son activité ludique se caractérise par cette nouvelle forme de raisonnement complexe et systématique qu'il peut mettre à profit dans différents jeux de règles complexes.

Le jeu symbolique est cette forme de jeu où l'enfant fait comme si. Il a le loisir de donner forme et vie à tous les objets qui se trouvent dans son contexte de jeu, c'est lui le metteur en scène, le réalisateur, le scripteur et l'acteur du scénario. Tout lui appartient, tout peut devenir autre, et tout peut être différent de ce qu'il avait prévu au départ. Bref, le jeu symbolique est un jeu du possible et du permis.

Selon l'approche piagétienne, le jeu symbolique se déroule en deux mouvements spécifiques. Un premier mouvement, entre deux à quatre ans, est caractérisé par l'imitation de soi, c'est-à-dire de ses propres gestes sur des objets (projection des schèmes symboliques sur des objets nouveaux). Un deuxième mouvement du jeu symbolique s'amorce entre quatre et sept ans, dû à une sorte de maturité symbolique acquise auparavant par l'acceptation et l'intériorisation de certaines données extérieures. Il est à noter que les enfants qui fréquentent la classe maternelle se situent dans cette catégorie. 
L'enfant de cinq ans qui fréquente la classe maternelle se situe dans une période particulièrement féconde sur le plan symbolique. Ayant agi jusqu'à maintenant directement sur les objets et ce, à l'aide de ses divers schèmes perceptifs et moteurs, il appréhende la réalité avec une pensée où domine la fonction symbolique. À cet âge, cette fonction symbolique se manifeste de diverses façons mais surtout par le jeu symbolique. Les autres modes qui permettent d'apprécier aussi cette pensée représentative ou symbolique sont l'imitation différée, l'image mentale, le symbole, le dessin et le langage.

À cet âge également, le jeu symbolique s'enrichit de trois nouvelles caractéristiques: un ordre relatif dans la construction ludique, un souci croissant de vraisemblance et d'imitation exacte du réel et un début de symbolisme collectif avec différenciation et ajustement des rôles (Piaget, 1978, pp.143-145).

Ces trois nouveaux caractères sont présents dans le jeu symbolique de l'enfant grâce aux progrès de sa pensée. Les outils de l'intelligence se raffinent progressivement et, à cet âge, «la pensée intuitive est une sorte de pensée imagée qui conduit à un rudiment logique; elle est assez souple pour permettre l'anticipation de résultats et l'établissement de combinaisons logiques simples; mais elle est encore incapable de véritables opérations logiques.» Toutefois, les caractéristiques même du stade intuitif mettent en évidence la limite du raisonnement de l'enfant de cet âge, car celui-ci tend à se concentrer sur un seul aspect d'une situation et néglige les autres dimensions présentes. Il est donc difficile pour l'enfant de considérer le point de vue des autres, à plus forte raison s'il est différent du sien. Il est possible d'observer, dans le jeu de l'enfant, la manifestation de chacune de ces caractéristiques.

L'ordre relatif dans la construction ludique, ou une certaine cohérence dans le jeu, s'observe en relevant la trame évolutive du scénario (gestes et paroles) de l'enfant. Le jeu possède une organisation et une structure interne spécifique. L'enfant détient une meilleure gestion des rôles, des actions, des paroles et des objets qui s'inscrivent dans le scénario. L'activité symbolique a un début, un déroulement et une fin, et la qualité d'ordre de la trame, entre le début et la fin, se manifeste selon les connaissances de l'enfant quant au scénario exploité. 
Le souci croissant de vraisemblance et d'imitation exacte du réel ou le souci croissant de copier la réalité avec exactitude et de se conformer le plus possible aux modèles imités se raffine au fur et à mesure qu'augmentent les connaissances et la compréhension de l'enfant envers la réalité imitée. En fait, dans son jeu symbolique, il nous livre des messages qui transmettent à la fois ses connaissances de la réalité, c'est-à-dire les éléments qui la composent, ses rapports avec cette réalité et la façon dont il se situe dans cette dynamique.

Dans le cas du symbolisme collectif, l'observation des enfants qui jouent a révélé jusqu'à maintenant une forme de jeu plutôt solitaire et parallèle. Les enfants jouent plus souvent ensemble mais avec des statuts bien individuels, sans trop se préoccuper du jeu de l'autre, si ce n'est que pour demander un objet, s'informer de ce que l'autre fait ou pour une conversation hors contexte du jeu. Vers cinq-six ans, on observe davantage une propension à un jeu plus social, où l'activité ludique passe du symbolisme individuel au symbolisme collectif. L'enfant commence à prendre plaisir à jouer avec l'autre et ce, dans un but commun. Les enfants parviennent progressivement à jouer à deux et à plusieurs, pour élaborer ensemble un projet commun, où le rôle et la tâche de chacun sont déterminés au préalable: «moi je fais le père, toi la mère et toi le chien.» Ces jeux à plusieurs sont le témoignage d'un net progrès de la socialisation ou de la coordination des points de vue ainsi que d'une diminution de l'égocentrisme enfantin. Progressivement, l'enfant est capable de se décentrer de son propre point de vue et d'accepter les idées des autres.

Ces trois caractéristiques colorent d'une façon bien particulière le jeu symbolique qui témoigne de la spécificité du jeu de l'enfant de quatre-sept ans. De là, émergent deux réflexions par rapport à la problématique de cette recherche. La première se situe dans le prolongement de la classification du jeu de Piaget et interroge la place et la fonction du jeu de construction, en l'occurrence la place et la fonction du matériel bloc dans cette classification. Pour sa part, la deuxième réflexion porte sur la troisième caractéristique du jeu symbolique, soit le symbolisme collectif. Il interroge en fait le concept de jeu symbolique social identifié par Smylansky (1968) quand elle parle du jeu à deux et à plusieurs. Cette forme nouvelle de participation sociale questionne toute la place de la dimension sociale comme élément structurant du jeu symbolique et comme élément signifiant dans l'élaboration cognitive. Elle remet en question la place du social dans la hiérarchie proposée par Piaget, soit de l'individuel au social. Est-ce que le jeu symbolique social se manifesterait 
beaucoup plus jeune que ne laisse supposer Piaget? Pour que s'élabore un jeu symbolique social, quelles sont les compétences cognitives et sociales que l'enfant met à contribution? Voilà les questions soulevées par l'analyse des positions piagétiennes.

\section{- La place des jeux de construction dans la classification de Piaget}

Selon Piaget, les jeux de construction ne constituent pas une catégorie située sur le même plan que les autres mais bien une forme frontière reliant les jeux aux conduites non ludiques.

Dans une classification génétique fondée sur l'évolution des structures, l'exercice, le symbole et la règle sont trois étages successifs qui caractérisent, d'une part, les grandes classes de jeux et, d'autre part, les diverses formes successives de l'intelligence, soit l'intelligence sensori-motrice, représentative et réfléchie. Selon ce point de vue, les jeux de construction ne définissent pas un étage spécifique mais marquent une transformation interne de la notion de symbole dans le sens de la représentation adaptée et occupent au deuxième niveau et surtout au troisième, une position située à mi-chemin entre le jeu et le travail intelligent ou entre le jeu et l'imitation (Piaget, 1978).

Et en poursuivant la réflexion de Piaget, Bühler interroge la distinction des jeux de fiction et des jeux de construction, car il y a une infinité d'intermédiaires entre une transformation symbolique d'un morceau de bois en une maison et une reproduction fidèle et objective d'une maison à l'aide du matériel bloc. Le jeu de construction constitue donc une catégorie particulière à la fois intermédiaire entre le jeu sensori-moteur et le jeu symbolique, ainsi qu'entre les deux et l'activité adaptée (adaptation simultanément pratique et représentative). Construire une maison avec des blocs est à la fois œuvre d'habileté sensori-motrice et de représentation symbolique et c'est dépasser le jeu proprement dit dans la direction du travail ou tout au moins de l'acte gratuit de l'intelligence (Piaget, 1978).

Cette position de Bühler et de Piaget à propos des jeux de construction soulève un questionnement intéressant quant aux potentialités spécifiques du matériel bloc dans la classe maternelle. De par le mode de fonctionnement de la pensée de l'enfant de cinq ans et de par son actualisation dans le jeu symbolique, nous sommes portée à attribuer au matériel bloc et à l'activité qu'il suscite une fonction et un rôle particuliers dans le jeu sym- 
bolique. Dans le contexte de la classe maternelle, dans l'espace réservé à l'activité avec le matériel bloc, l'observation des enfants nous révèle des types de comportements suscités par ce matériel. Celui-ci engendre d'abord une première forme particulière de représentation reliée à l'activité dite de construction de bâtiments de tous genres tels que châteaux, hôpitaux pour animaux, garage à étages, intérieur de maison, maison avec piscine à l'élaboration de villes, de systèmes routiers et de moyens de transport. Par la suite, une deuxième forme de représentation est reliée à l'élaboration de scénarios, tantôt simultanée à l'activité de construction, tantôt subordonnée aux produits de cette activité. L'activité dite de construction de la première forme de représentation pourrait être comparable au dessin mais il est plutôt rare que les représentations dans le dessin donnent naissance à l'élaboration de scénarios. L'enfant va plutôt parler d'une scène qu'il a représentée ou apporter des éléments explicatifs de la situation.

Ainsi, le matériel bloc serait un médium qui permettrait à l'enfant d'exprimer un type particulier de jeu symbolique, avec des formes de représentations à divers niveaux tels que représentations matérielles et représentations symboliques entrainnant des transformations matérielles et symboliques de plus en plus complexes. Il serait en fait un matériel exploitant davantage une facette ou une dimension du jeu symbolique par l'activité qu'il suscite.

Après avoir tracé les grandes lignes de la pensée de Piaget sur la conception de la connaissance, du jeu et du jeu symbolique, examinons maintenant la pensée de Vygotsky.

\subsubsection{Vygotsky, une conception du développement de l'intelligence et des connaissances où l'aspect social est central}

Préoccupé par le développement et le fonctionnement des processus mentaux supérieurs, les travaux de Vygotsky (1966) reposent sur une conception du développement de l'enfant dans laquelle le social et l'activité sont des concepts centraux.

Évidemment, pour mieux comprendre la position de Vygotsky, il est important de se rappeler que ses différentes présuppositions considèrent que les processus mentaux supérieurs sont formés socialement et sont transmis culturellement tout au long de la vie d'un individu, les dispositions biologiques étant rapidement remplacées par les forces culturelles s'actualisant à travers les efforts individuels dans l'activité pratique et à travers 
un système de relations sociales à l'intérieur duquel l'activité se produit (Rubin, Fein et Vandenberg, 1984).

Élaborée presqu'à la même époque que Piaget, Vygotsky propose une théorie du développement cognitif où l'aspect social est central et où il considère le rôle de la société et des interactions sociales comme des médiateurs premiers et essentiels dans le développement des processus mentaux supérieurs et reléguant beaucoup plus tard la contribution individuelle dans l'élaboration de ces processus.

Ce déplacement de l'emphase de l'individuel au social permet à Vygotsky d'attribuer une importance première au contexte social dans le processus du développement cognitif. L'enfant est incrusté dans le milieu social et reflète ce milieu. Toute la connaissance de l'enfant est d'abord issue du domaine social et plus tard, elle devient internalisée et incorporée à l'intérieur de l'organisation cognitive (cette internalisation demeure quand même une notion confuse et très peu explicite dans les écrits de Vygotsky, comparable à la notion confuse d'interactions sociales dans les écrits de Piaget). Les fonctions sont premièrement interpsychologiques et plus tard intrapsychologiques. Pour comprendre l'organisation cognitive de l'enfant, on doit d'abord comprendre la structure et le contenu du monde social de l'enfant (Vygotsky, 1978).

Pour Vygotsky, les structures mentales sont construites à partir de l'utilisation d'outils et de signes. Les outils se développent dans les actions pratiques de l'individu, de l'extérieur sur l'environnement matériel. Par exemple, il considère le langage comme un outil (l'outil le plus avancé que nous ayons) et la conscience comme un autre outil. Il va de soi que les règles qui régissent l'emploi des outils dans différents domaines d'action sont données à travers un code social qui constitue la pratique d'une culture, une espèce de boîte à outils de la culture. Ce n'est pas que le comportement soit déterminé par la culture mais que la culture fournisse le cadre d'interprétation au comportement. Ainsi les processus mentaux supérieurs représentent la fusion des outils utilisés et des signes utilisés.

En plus de la notion d'outil et de signe constituant l'élaboration des processus cognitifs, Vygotsky, préoccupé par les processus interactifs (interactions sociales), identifie la notion de zone proximale du développement. Il définit cette zone comme «la distance 
entre le niveau de développement actuel, tel qu'on peut le déterminer à travers la façon dont l'enfant résout seul des problèmes et le niveau de développement potentiel, tel qu'on peut le déterminer à travers la façon dont l'enfant résout des problèmes lorsqu'il est assisté par l'adulte ou collabore avec d'autres enfants plus avancés.» Cette zone proximale de développement est en fait la période où un développement est le plus susceptible de s'actualiser significativement pour l'enfant.

\subsubsection{Vygotsky, une conception du jeu et du jeu symbolique à tra- vers le filtre psychosocial où l'aspect cognitif est central}

De prime abord, la lecture de la position de Vygotsky sur le concept de jeu et de jeu symbolique n'est pas simple dans le contexte où celui-ci a élaboré sa théorie en considérant des variables sociales. Selon sa conception, les besoins, les tendances, les objectifs

et les motifs sont à la base de l'activité de l'enfant. À chaque âge, des besoins et des objectifs spécifiques se manifestent et ceux-ci sont toujours très importants dans le développement global. L'enfant très jeune voit généralement ses besoins immédiatement satisfaits lorsqu'ils sont exprimés. L'âge préscolaire engendre un certain nombre de besoins et d'objectifs spécifiques qui ne peuvent être satisfaits ou réalisés immédiatement. Ainsi s'installe un intervalle toujours un peu plus long entre les besoins, les désirs, les motifs et leurs réalisations. L'enfant doit trouver une façon de réduire cet intervalle entre les désirs et leurs réalisations et le jeu deviendrait le moyen de réduction de cet intervalle. Car pour résoudre les tensions, l'enfant pénètre «dans un monde imaginaire et illusoire dans lequel les désirs irréalisables peuvent être réalisés.» (Vygotsky, 1967, p.7). Le jeu émerge donc des tensions entre les désirs qui ne peuvent être ni oubliés par l'enfant, ni réalisés par la société. Dans cette ligne de pensée, ce sont les objectifs affectifs en terme de désirs irréalisables qui conduisent au jeu; ils sont le commencement, qui nécessite la création d'une situation imaginaire.

Ainsi le jeu est vu comme une formation comportementale hautement motivée. Cette motivation provient de la tension de la vie réelle. Il est un mécanisme adaptatif promouvant la croissance cognitive pendant les années préscolaires (Vygotsky, 1967, 1978). En l'occurrence, le jeu origine de pressions affectives et sociales et non pas de facteurs cognitifs. Il est essentiellement le désir d'accomplissement des objectifs, non pas de désirs isolés, mais d'affects généralisés. Le jeu est donc considéré comme une création de 
l'enfant d'une zone proximale de développement qui est le plus haut niveau de performance dont il est capable.

Dans le contexte de la théorie vygotskienne, le jeu est toujours symbolique, parce qu'à la base, pour qu'il y ait jeu, il faut qu'il y ait la création d'une situation imaginaire. Au contraire, d'autres points de vue où la situation imaginaire est un attribut d'un groupe donné d'activités de jeu, ici la situation imaginaire est un attribut critérié du jeu en général. Ainsi, c'est de cette possibilité de créer une situation imaginaire qui permet de distinguer l'activité de jeu des autres formes générales d'activités. Par contre, seuls les enfants, à partir de trois ans, possèdent les rudiments nécessaires à la création d'une situation imaginaire. Il semble donc que l'imagination soit une nouvelle organisation ou disposition de l'enfant qui représente une forme spécifique de l'activité consciente. L'enfant serait donc capable de créer une situation imaginaire à partir du moment où il commence à établir une différence entre le champ de la perception (visuelle) et le champ de la signification.

Comment se crée cette situation imaginaire et comment l'enfant se comporte-t-il? La situation imaginaire en elle-même contient des règles qui, d'une certaine façon, orientent et imposent des comportements. Comme ces règles intrinsèques à cette situation imaginaire ne sont formulées à l'avance et ne changent pas au cours du jeu, elles excluent en conséquence un certain nombre de possibilités réelles pour l'action. Ainsi, pour Vygotsky, toutes les situations imaginaires contiennent des règles sous une forme cachée et tous les jeux avec des règles contiennent une situation imaginaire sous une forme cachée.

Mais d'où viennent ces règles de comportement qui sont réinvesties dans le jeu? Pour résoudre cette question, Vygotsky fait référence à la partie des travaux de Piaget consacrée à l'étude des règles de jeu dans ce que cet auteur appelle le développement moral de l'enfant. Ces travaux mettent en évidence deux sources distinctes du développement des règles de comportement qui à un moment apparaissent dans le jeu; ces règles proviennent d'une collaboration mutuelle entre l'adulte et l'enfant ou entre les enfants eux-mêmes. 
Une fois la situation imaginaire créée, que se passe-t-il? À partir de trois ans, une nouvelle forme de comportement est rendue possible en ce qui a trait à la libération des contraintes situationnelles quand l'enfant agit dans une situation imaginaire. Dans la situation réelle, le très jeune enfant est limité dans toutes ses actions par les contraintes situationnelles et par le champ de la perception (visuelle), seul champ utilisé pour toutes ses actions. Il devient capable d'imaginer dans sa tête une situation, indépendamment de la situation concrète immédiate. C'est le transfert qui se produit dans le jeu. L'enfant apprend à se comporter dans un domaine cognitif plutôt que dans un domaine visible extérieur. Ainsi, l'action de l'enfant dans la situation réelle se distingue de l'action de l'enfant dans la situation de jeu, par le fait que dans la première, son comportement est guidé par la perception immédiate des objets ou par la situation immédiatement affectée par lui. Tandis que dans la deuxième situation, l'action dans la situation imaginaire est une action conçue, imaginée qui guide son comportement par la signification de la situation. Ainsi, pour l'enfant de plus de trois ans, il est possible de séparer le domaine de la perception du domaine de la signification. C'est donc à cet âge que la première divergence entre le domaine de la perception et celui de la signification apparaît.

Dans cette ligne vygotskienne, la rupture qui s'établit entre le domaine de la perception et le domaine de la signification est un point crucial de la conception du jeu symbolique. Dans le jeu, la pensée est séparée des objets et l'action découle des idées plutôt que des choses. Le jeu est donc un stade, une étape et une phase transitionnelle dans cette voie de rupture. Il serait un moyen pour l'enfant de parvenir progressivement à cette rupture des domaines de la perception et de la signification.

En somme, selon cette conception vygotskienne du développement de l'enfant, la création d'une situation imaginaire peut être vue comme une intention en voie de développement de la pensée abstraite. Le jeu est donc réellement une caractéristique de l'âge préscolaire.

L'examen des conceptions et positions de Piaget et de Vygotsky en ce qui concerne le développement de la connaissance de l'enfant, du jeu et du jeu symbolique nous amène à investiguer les différentes recherches sur le jeu symbolique qui se sont réalisées à partir de l'un ou l'autre de ces cadres de référence, afin de mettre en évidence les éléments 
théoriques qui ont émergé et qui ont contribué à une meilleure connaissance et compréhension du jeu symbolique.

\subsubsection{Vers un renouveau de l'intérêt pour le jeu symbolique}

Pendant les deux dernières décennies, la recherche manifeste un renouveau d'intérêt pour le jeu symbolique. La consultation des écrits dans ce domaine est un indicateur quantitatif important de l'abondance et de la croissance des études entreprises sur ce sujet. Cette nouvelle période de recherche sur le jeu symbolique trace une orientation tout à fait différente de l'ensemble des études réalisées auparavant et axées principalement sur le contenu du jeu des enfants. En effet, l'investigation des chercheurs provenant du domaine de la psychologie s'oriente davantage sur le rôle du jeu symbolique dans le développement cognitif et dans l'évolution de la personnalité globale de l'enfant. Dès l'apparition des premières formes de manifestations de ce type de jeu dans la petite enfance jusqu'à la fin du préscolaire et du début de l'élémentaire, les préoccupations de la recherche contemporaine se concentrent sur les composantes structurales du jeu, les changements réalisés au cours de l'évolution même du jeu symbolique, sur les facteurs d'influences (facteurs familiaux, environnementaux, socio-économiques et culturels), sur les différences individuelles, etc.

De ces études, il importe de retenir les nouveaux éléments explicatifs et de compréhension du jeu symbolique qui émergent et qui viennent successivement et simultanément raffiner, compléter, remettre en question, dépasser et proposer d'autres avenues afin d'appréhender toujours un peu plus le jeu symbolique dans sa dynamique et sa complexité. Rubin, Fein et Vandenberg (1984) proposent une synthèse des recherches sur le jeu symbolique et font état d'une pluralité de chercheurs impliqués dans cette thématique.

Rappelons que traditionnellement la recherche sur le jeu symbolique s'est réalisée sans distinction de l'évolution de ce jeu. À cet effet, Piaget et Vygotsky ont effectué leur analyse des fonctions du jeu symbolique sans différencier l'activité symbolique solitaire de l'activité symbolique sociale. Cependant, en 1968, Smilansky introduit pour la première fois une distinction importante entre le jeu dramatique et le jeu sociodramatique. Le jeu dramatique est cette forme particulière de jeu où l'enfant traduit sa connaissance et son expérience du monde en jouant le rôle d'un personnage et en imitant ses gestes et paroles 
avec ou sans objets. Le jeu sociodramatique intègre en plus la présence et la complicité d'un ou plusieurs partenaires dans l'élaboration des scénarios. Pour que le jeu sociodramatique prenne vie, il faut s'assurer de l'imitation de personnages, de la présence de divers éléments de faire-semblant et de l'interaction sociale sous forme de coopération, de négociation, d'explication, etc.

De l'ensemble de ces recherches, la synthèse réalisée par Rubin, Fein et Vandenberg (1984) dégage cinq composantes structurales du jeu de faire-semblant dans des cadres de recherche en situations naturelles. Chacune de ces propriétés structurales vient alimenter et regrouper les diverses recherches sous des balises synthèses qui témoignent de l'avancement des connaissances théoriques et expérimentales dans le domaine du jeu symbolique. Ces propriétés structurales sont la décontextualisation et la substitution d'objets; le jeu social ritualisé, l'adoption de rôles, le jeu thématique fantaisiste/le jeu sociodramatique et enfin, la structuration du jeu. Ces propriétés structurales seront réinvesties plus en profondeur dans la partie méthodologique de cette recherche.

En somme, les différents éclairages apportés par les travaux de Piaget, de Vygotsky et des divers auteurs qui ont travaillé sur la thématique du jeu symbolique fournissent des éléments théoriques et méthodologiques pertinents pour analyser et comprendre la dynamique de l'activité de l'enfant avec le matériel bloc.

Dans le contexte de la classe maternelle québécoise (enfant de cinq ans) et de l'espace réservé au matériel bloc, l'activité de construction et l'activité de jeu symbolique suscitées par ce matériel sont de plus en plus des activités qui se réalisent à deux, trois ou quatre enfants, donc des activités qui s'organisent en situation de collaboration et de coopération entre des partenaires de jeu.

De ce fait, il nous apparaît qu'un ensemble d'éléments essentiels dans la structure de l'activité de l'enfant avec le matériel bloc se situe sur le plan interactionnel, c'est-à-dire sur le plan des interactions sociales entre les enfants. Ces éléments influenceraient et détermineraient l'activité de construction et de jeu symbolique simultanée ou subordonnée à l'activité de construction. 
Par conséquent, il est important d'examiner la place, le rôle et l'apport des interactions sociales dans différentes conceptions du développement de l'enfant afin de dégager une compréhension significative des interactions sociales dans l'activité des enfants avec le matériel bloc.

\subsection{Vers une compréhension des interactions sociales; du constructivisme au socio-constructivisme: Piaget, Vygotsky, Doise, Mugny et Perret- Clermont}

Dans le cadre de cette recherche, nous avons retenu les conceptions du développement de l'intelligence de Piaget (1964), de Vygotsky (1978), de Doise et Mugny (1981) et de Perret-Clermont (1979) qui permettent, d'une part, de mettre en lumière la spécificité des interactions sociales dans chacun de ces contextes d'explications et de compréhension du développement et, d'autre part, de saisir l'évolution de ce concept en fonction de l'importance de son rôle dans le développement.

\subsubsection{Piaget, le rôle des interactions sociales dans sa conception du développement de l'intelligence}

Rappelons tout d'abord que la conception de Piaget, quant au développement, se centre sur l'individu, ses potentialités et sur le facteur d'équilibration et de régulation interne de tous les possibles de l'individu. Dans sa conception du développement de la connaissance, Piaget privilégie l'aspect individuel dans le développement cognitif. Sa position est claire, les constructions cognitives sont l'œuvre de mécanismes psycho-individuels et elles sont le résultat de confrontations intra-individuelles. Tout se passe à l'intra, c'est-à-dire à l'intérieur du sujet, c'est toujours une activité interne qui prend sa source aux niveaux de l'action et de la pensée de l'individu lui-même. C'est essentiellement le sujet qui est responsable de la régulation de ces mécanismes, c'est-à-dire d'assurer le fonctionnement et de maintenir l'équilibre.

Dans cette façon de concevoir le développement de l'intelligence, Piaget identifie plusieurs facteurs qui y contribuent. Il s'agit de la maturation organique et neuropsychique, de l'expérience physique et logico-mathématique (le rôle de l'exercice et de l'expérience que le sujet acquiert en agissant sur l'objet), des facteurs d'interaction et de 
transmission sociale, de l'affectivité et de l'équilibration. Parmi ces facteurs, seule l'équilibration représente, pour Piaget, le facteur essentiel du développement de l'intelligence. Dans cet ordre d'idées, le facteur social est un épiphénomène considéré comme un élément non structurant des constructions cognitives. Seule l'interaction de tous les facteurs permet de rendre compte de la formation des structures de l'intelligence.

Même si dans l'ensemble de ses travaux Piaget se centre exclusivement sur l'individu, il aborde également l'élément social en termes d'échanges sociaux, de relations sociales, d'influences sociales, de vie sociale et d'interactions sociales.

Sa description de l'évolution de l'enfant depuis son égocentrisme primitif conduit Piaget à parler de socialisation de l'intelligence individuelle et laisse entrevoir l'idée d'une pensée dont la nature originelle serait individuelle et qui deviendrait progressivement de plus en plus socialisée (Piaget, 1967).

Cependant, cet élément social est considéré dans un processus hiérarchique des interactions sociales se traduisant en termes de coopération, qui n'atteindrait son apogée qu'au stade des opérations concrètes et surtout formelles. Ce n'est qu'à ces niveaux que se poserait le problème des rôles respectifs de l'échange social et des structures individuelles dans le développement de la pensée. À ces stades, la spécificité des interactions sociales se manifesterait dans l'aptitude à coopérer et cette coopération serait solidaire du développement des opérations, également tributaire de la nature à la fois sociale et individuelle de la logique.

À chaque période du développement de l'enfant, soit de la période sensori-motrice, symbolique et intuitive et préopératoire, Piaget mentionne et reconnaît la présence d'influences sociales multiples et l'apparition de nouvelles relations sociales qui enrichissent et transforment la pensée de l'individu sans toutefois les considérer comme des agents de modifications profondes des structures de l'intelligence.

Mais, ce n'est qu'à la période des opérations concrètes et formelles, grâce à la constitution d'une logique et à la formation des rapports sociaux de coopération que se constitueraient des modifications profondes des structures de l'intelligence et qui permettraient alors de parler de pensée socialisée. 
Donc pour Piaget, il semble bien que pour être capable de coopérer, les partenaires doivent avoir atteint un certain seuil de développement, seuil qu'il identifie et associe au stade des opérations concrètes. De plus, pour que cette coopération bénéficie à chacun des partenaires en situation, il met l'accent sur l'importance des interactions entre partenaires se considérant comme égaux (égalité qui renvoie à un rapport non hiérarchique, comme par exemple enfant-adulte).

La coopération consiste elle-même en un système d'opérations, de telle sorte que les activités du sujet s'exerçant sur les objets et les activités des sujets lorsqu'ils agissent les uns sur les autres se réduisent, en réalité, à un même système d'ensemble dans lequel l'aspect social et l'aspect logique sont inséparables dans la forme comme dans le contenu. (Piaget, 1965, p.90)

\subsubsection{Vygotsky, le rôle des interactions sociales dans sa conception du développement de l'intelligence}

Vygotsky, dans sa conception du développement, se centre sur le social. Il accorde une importance primordiale aux facteurs sociaux dans les processus de développement. Étant un être résolument social et ce, dès sa naissance, l'individu est d'abord imprégné de tout un système de relations et de régulations interpersonnelles qui le constituent et que l'individu s'affaire à transformer tout au long de sa vie en un système intrapersonnel. C'est d'abord à travers ses interactions avec les autres que l'individu découvre son environnement et apprend à agir sur les objets et à utiliser les systèmes de significations, principalement le langage, pour la régulation progressive de ses activités et de son comportement interpersonnel, et, plus tard, à réguler son comportement personnel et ce, par intériorisation. L'intériorisation du processus interpersonnel constitue l'œuvre de l'individu qui, à son achèvement, se transforme en processus intra-personnel et constitue l'intra-psychologique. Sa position est claire: «les fonctions supérieures débutent comme des relations effectives entre individus humains.» (Vygotsky, 1978, p.57) et sont le résultat de confrontations interindividuelles. Tout se passe d'abord à l'inter, c'est-à-dire à l'interindividuelle. C'est toujours une activité qui prend sa source au niveau des relations et des régulations interpersonnelles.

Pour Vygotsky, c'est par, avec et dans les interactions sociales que l'individu se construit progressivement. Il n'établit ni de nature spécifique, ni de processus hiérarchique des interactions sociales. Cependant, il identifie comme étant nécessaire que les 
interactions se réalisent avec des partenaires plus compétents, c'est-à-dire des adultes. Pour lui, le développement cognitif s'avère tributaire des interactions sociales, particulièrement dans les situations fortement asymétriques, adulte-enfant. Les progrès réalisables grâce au soutien de l'adulte dépendraient de la zone de développement proximal, c'est-àdire que les acquisitions dépendraient à la fois du niveau de l'enfant déjà atteint et de l'apport d'une aide instrumentale juste nécessaire.

\subsubsection{Doise, Mugny, Perret-Clermont, le rôle des interactions so- ciales dans la conception du développement de l'intelligence}

En psychologie sociale, le concept d'interaction a toujours été présent de manière plus ou moins explicite. Depuis quelques années, il est devenu d'usage de dire que l'interaction sociale constitue l'objet d'étude de la psychologie sociale. Cependant, en rapport avec les traditions et les différentes orientations de la psychologie sociale,

plusieurs représentations de l'objet psychosociologique se dégagent et aboutissent à poser plusieurs courants en psychologie sociale.

Dans le prolongement de certaines préoccupations, notamment eu égard au développement de l'intelligence, et pour le propos de cette recherche, c'est la psychologie sociale génétique qui a retenu plus particulièrement notre attention en fonction du rôle des interactions sociales dans le développement cognitif de l'individu.

La psychologie sociale génétique est une conception psychosociale du développement psychologique individuel (Mugny, 1985). Elle propose une perspective théorique intégrative des aspects individuel et social du développement cognitif. Elle tente une insertion du social et élabore explicitement les mécanismes constitutifs et structurants des processus interpersonnel et intrapersonnel. Cette élaboration des mécanismes semble se réaliser selon une empreinte explicite des mécanismes proposés par Piaget dans l'explication du développement de la connaissance et du développement cognitif. Elle place au premier rang la dimension sociale du développement cognitif dans la compréhension du rôle de l'expérience sociale en tant que mécanisme même des constructions cognitives individuelles.

Elle reconnaît bien évidemment que le développement cognitif consiste en une structuration progressive des rapports avec l'environnement qui est aussi indivi- 
duelle. Cependant, ces dynamiques individuelles sont conçues comme se fondant sur des expériences sociales qu'elles sont amenées à structurer. Ces expériences sociales ne constituent pas alors autant de facteurs qui se grefferaient à titre de sources accidentelles (voire aléatoires) sur l'ontogenèse, mais interviennent à titre de constituants de ces dynamiques individuelles. (Mugny, 1985, p.18).

La psychologie sociale génétique propose donc, en quelque sorte, une perspective théorique cohérente et unifiante qui ne fait pas de rejet en bloc des éléments du constructivisme piagétien, mais qui vise:

à dégager quelques mécanismes centraux (voire un mécanisme psychosocial central) par lesquels (ou par lequel) le social est générateur de progrès dans le développement cognitif individuel; ou, pour être plus précis encore, par lesquels (ou par lequel) le social participe, en tant que nécessaire constituant, à la construction des habiletés cognitives individuelles. (Mugny, 1985, p.10).

Piaget et Vygotsky demeurent deux penseurs marquants de la psychologie sociale génétique. Ce que la psychologie sociale génétique semble retenir de la conception de Piaget est certes son épistémologie constructiviste et interactionniste, puisqu'il a su habilement, dans ses explications du développement de la connaissance et du développement cognitif, être des plus exhaustifs et explicites sur les aspects structuralistes de ce développement.

La psychologie sociale génétique reprend à Piaget l'idée que c'est en agissant sur le milieu environnant que l'individu élabore des systèmes d'organisation de cette action sur le réel et insiste sur le fait que la causalité attribuée à l'interaction sociale n'est pas unidirectionnelle, mais circulaire, voire complexe. (Hinde, PerretClermont et Stevenson-Hinde, 1988, p.445).

Même si on lui attribue beaucoup d'implicite en regard de l'aspect social, et même s'il a élaboré davantage sur cet aspect dans l'étude du développement moral de l'enfant, il demeure essentiellement individuel dans le social, en ne considérant ce dernier qu'à titre d'épiphénomène nécessaire mais non comme élément constituant et structurant du développement cognitif. La seule insistance importante est, sans doute, sur le rôle des interactions sociales, notamment avec des pairs, dans le développement cognitif et social (Piaget, 1932, 1964, 1970).

Ce que la psychologie sociale génétique semble retenir de la conception de $\mathrm{Vy}$ gotsky est certes son postulat qualifié d'intuitif et d'essentiel à son approche: 
un processus interpersonnel se transforme en un processus intra-personnel. Chaque fonction apparaît deux fois dans le développement culturel de l'enfant: d'abord au niveau social, et ensuite, au niveau individuel; d'abord entre individus (interpsychologique) et, ensuite, dans l'enfant (intrapsychologique).» (Vygotsky, 1978, p.57).

Cependant, même s'il avance l'hypothèse affirmée d'une causalité allant de manière privilégiée du social au cognitif, il demeure très peu explicite quant aux processus qui soustendent cette causalité. Il conçoit le développement cognitif comme largement dépendant de l'intériorisation de compétences qui se manifestent en premier lieu dans l'interaction avec autrui plus compétent.

Présentement la psychologie sociale génétique emprunte largement au modèle interactionniste de Moscovici (1976) et de la notion-clé de conflit, comme élément structurant, source de changement chez l'individu et dans les systèmes sociaux (Mugny, 1985).

Plus particulièrement, la psychologie sociale génétique ou la théorie psychosociale du développement de l'intelligence de Doise, Mugny et Perret-Clermont propose une autre définition de l'intelligence intégrant à la conception piagétienne la composante sociale. Cette définition est fondée sur la thèse générale selon laquelle « les coordinations entre individus sont à l'origine des coordinations individuelles, qu'elles les précèdent et les génèrent.» (Doise et Mugny, 1981, p.34). Ces coordinations interindividuelles joueraient donc un rôle causal dans le développement cognitif.

L'objectif primordial des psychosociologues du développement consiste à mettre en évidence les mécanismes psychosociaux du développement de l'intelligence et à les analyser. Dans leur modèle explicatif, ils intègrent les variables sociales comme éléments constitutifs, au même titre que les variables psychologiques selon le schéma proposé par Moscovici (1984), qui réalise le passage d'une psychologie bipolaire (Ego-Objet) à une psychologie tripolaire (Ego-Alter-Objet). Cette théorie psychosociale du développement de l'intelligence a donc pour objet d'étude la construction sociale des représentations cognitives et sa tâche consiste essentiellement à la spécification des différentes formes d'interactions sociales plus complexes menant à de nouveaux progrès.

Mettre en évidence que l'interaction sociale joue un rôle causal dans le développement cognitif n'implique pas une épistémologie sous-jacente concevant l'individu 
comme passivement façonné par des processus extérieurs. Au contraire, nous envisageons le sujet dans son activité, au sein d'un environnement qui est toujours simultanément social et physique et dans lequel la présence, dès sa naissance, d'autres individus attacheront à le démontrer, que c'est au travers de ces coordinations avec autrui que l'enfant est amené à élaborer des systèmes d'organisation de ses actions sur le réel. C'est dans ces conditions de coordinations interindividuelles qu'il cherchera à maîtriser que l'individu par un mécanisme d'abstraction élaborera ses structures cognitives. En retour, ses progrès cognitifs permettront au sujet de participer à des interactions sociales nouvelles plus élaborées, qui à leur tour modifieront la structuration de sa pensée. Le lien causal supposé est circulaire et sa progression correspond à la spirale tracée par Piaget pour décrire la section du développement. (Perret-Clermont, 1986, p.36)

La psychologie sociale génétique repose sur un socio-constructivisme tripolaire qui enrichit la vision bipolaire piagétienne du sujet et de son interaction avec l'objet en accordant une place privilégiée à autrui dans le mouvement de l'interaction de l'autre, dans l'appréhension de l'objet et dans l'interaction de cet environnement humain et physique au soi. Le constructivisme social, berceau épistémologique de la psychologie sociale génétique, intègre l'idée de l'individu qui construit sa connaissance. C'est lui le responsable de l'appropriation, de l'organisation, de la gestion de la connaissance ainsi que de l'idée de l'imprégnation constituante et structurante de l'autre et de son rôle déterminant dans cette construction de la connaissance.

Pour comprendre l'essence de cette conception, des concepts-clés tracent les balises en fournissant les explications des mécanismes qui opèrent dans le développement cognitif à teneur sociale. Ces concepts sont l'interaction sociale et la signification sociale, le conflit socio-cognitif et les conditions d'émergence et enfin, le marquage social.

L'interaction sociale et la signification sociale sont deux concepts-clés qui animent la présence de la dynamique sociale dans le processus de construction des savoirs et assurent la compréhension, l'explication et la répartition des divers courants théoriques qui précisent la nature des dimensions sociales. Dans la conception psychosociologique génétique élaborée par Doise, Mugny et Perret-Clermont, les interactions sociales constituent un élément-clé en regard de la nature des dimensions sociales et dont le rôle est envisagé comme générateur de changement. Toujours selon cette approche, les significations sociales sont appréhendées en fonction des contenus mêmes des tâches à résoudre, notamment par le concept de marquage social. 
Le concept de conflit socio-cognitif n'est pas l'apanage exclusif de la psychologie sociale génétique, la théorie piagétienne parle de conflit psychologique comme «résultant des confrontations et des contradictions entre les actions ou les anticipations du sujet et les observables ou les résultats de son action.» (Inhelder, Sinclair et Bovet, 1974: voir Gilly, 1989, p. 166).

Le conflit est considéré, selon le cas, comme source potentielle de progrès cognitif ou de perturbation. La source de ces conflits est généralement imputée à diverses propriétés de stimuli, telles que leur nouveauté, leur ambiguïté, pouvant effectivement aboutir à un conflit entre réponses symboliques incompatibles, et entraînent un comportement exploratoire visant à résoudre un tel "conflit conceptuel" du fait d'une activation émotionnelle et de l'éveil d'une sorte de "curiosité épistémique" ou à l'opposition entre les hypothèses émises par l'individu et les observables ou constatations qui les infirment ou induisent une satisfaction intellectuelle. (Carugati et Mugny, 1985, p.57).

Ce que l'on peut retenir de la définition psychologique du conflit, c'est qu'il survient de confrontations et de contradictions essentiellement de l'individu lui-même par rapport à ses représentations cognitives et ses actions. Il est de nature intra-individuelle et reste interne au sujet en ne considérant que la régulation interne qu'il suscite, donc il s'associe à la notion de déséquilibre dans l'esprit piagétien. Or, dans ce conflit, la dimension sociale en est exclue.

Pour sa part, le conflit socio-cognitif introduit la dimension sociale laissée pour compte dans les diverses approches de conflit et, avec Smedslund (1966), on lui reconnaît une efficacité plus grande lorsque celui-ci s'inscrit dans un conflit de communication sociale. Il puise sa source dans les interactions sociales et s'actualise dans un système de centrations cognitives opposées à propos du réel ou d'actions sur le réel. Les individus se trouvant ainsi en présence de confrontations et de contradictions, devront coordonner leurs différentes centrations afin de résoudre le conflit.

Ce sont dans et par les interactions sociales que les conflits socio-cognitifs naissent et dans et par les interactions sociales que les mêmes conflits se résolvent pour finalement se coordonner progressivement dans un mouvement qui va de l'interpersonnel vers l'intrapersonnel pour aboutir à l'élaboration d'instruments cognitifs nouveaux et plus avancés. Cependant, les interactions sociales ne semblent pas toutes empreintes d'un progrès socio-cognitif, certaines peuvent être plus efficaces pour induire le développement 
cognitif que d'autres. Cependant, il faut noter qu'il y a des moments du développement de l'enfant plus opportuns, et que la présence d'un certain type de partenaires particuliers n'est pas à négliger.

Dans ce sens, Doise, Mugny et Perret-Clermont identifient quatre conditions susceptibles de garantir un progrès socio-cognitif: l'hétérogénéité des niveaux cognitifs des partenaires, l'opposition des centrations, l'existence de points de vue opposés et la remise en question ou la méthode socratique. Ainsi,

les rencontres interindividuelles sont cognitivement structurantes et conduisent donc au progrès cognitif à la condition qu'elles assurent le déroulement d'un conflit socio-cognitif, d'une opposition sociale de réponses ou de points de vue à propos d'une tâche commune. (Carugati et Mugny, 1985, pp.64-65).

Le conflit socio-cognitif, comme source de progrès de la connaissance, permet, d'une part, la prise de conscience de l'existence de réponses possibles autres que les siennes et fournit, d'autre part, des informations qui peuvent aider à élaborer une nouvelle réponse. Il est donc,

[...] source de déséquilibre. Déséquilibre à la fois social et cognitif. Déséquilibre cognitif puisque le système cognitif n'est pas tel qu'il puisse intégrer à la fois ses propres réponses et celles d'autrui en un unique ensemble cohérent; il ne peut pas expliquer autrui et lui-même en même temps. Déséquilibre social puisque ce n'est pas simple désaccord cognitif: celui-ci s'inscrit dans des relations entre individus pour lesquels ce conflit pose un problème social. Sans ce problème social, l'enfant aurait peu de chances de ressentir un conflit cognitif. (Mugny, 1985, p.175).

La notion de marquage social ne s'inscrit pas dans la nature des dimensions sociales liée au rôle des interactions sociales mais plutôt dans celle liée au rôle des significations sociales. Cette notion ne repose donc pas sur des oppositions interindividuelles mais sur la signification sociale de la tâche. Elle prend en compte des types de mécanismes psychosociaux, autres que ceux qui relèvent d'interactions entre les individus différents afin d'expliquer le rôle de la dimension sociale dans la construction cognitive individuelle. Ces mécanismes psychosociaux originent dans ce cas du contenu même de la tâche et des situations. Ainsi, il y a marquage social

quand il y a homologie entre, d'une part, les relations sociales caractérisant l'interaction des protagonistes d'une situation spécifique et, d'autre part, les relations co- 
gnitives portant sur certaines propriétés des objets qui médiatisent les relations sociales. (Doise, Mugny, Perret-Clermont, 1975).

Cependant, dans cette recherche, seul le concept d'interaction sociale sera investigué pour tenter de comprendre la dynamique sociale dans l'activité de construction et celle du jeu symbolique.

\subsubsection{La recherche selon une perspective psychosociale}

Comme nous l'avons déjà mentionné, depuis quelques années, un courant socioconstructiviste émane et accorde aux interactions sociales une fonction beaucoup plus essentielle et structurante que ne l'avait considéré Piaget. Loin de rejeter les bases fondamentales de l'intelligence et de la connaissance, ce courant propose une perspective psychosociale pour expliquer l'organisation des instruments de connaissance.

Cette conception théorique du développement de l'intelligence a entraîné deux grands mouvements au sein de la recherche en rapport avec l'ensemble des approches qui concernent l'étude des mécanismes psychosociaux des constructions cognitives. Gilly (1989) a établi une classification de l'ensemble de ses travaux à partir de trois critères essentiels: la nature des situations et variables sociales dont le rôle est étudié, les types de compétences et fonctionnements cognitifs concernés et les ancrages théoriques adoptés.

Un premier mouvement regroupe des chercheurs qui interrogent le rôle des interactions sociales dans les constructions cognitives et un deuxième mouvement regroupe des chercheurs qui interrogent le rôle des significations sociales. Seuls ceux du premier mouvement seront examinés puisque notre intérêt se porte sur les interactions sociales.

Partant du premier critère de classification, ce premier mouvement de recherche est partagé entre les chercheurs selon qu'ils privilégient le caractère symétrique ou dissymétrique des statuts et des rôles assignés dans la situation proposée.

Dans une situation à caractère symétrique, deux courants théoriques départagent les chercheurs. Un premier courant théorique représenté par la psychologie sociale génétique et la théorie du conflit socio-cognitif de Doise, Mugny, Perret-Clermont (1974, 1975; 
Perret-Clermont, 1976; Mugny, Doise, Perret-Clermont, 1976) est issu des différents travaux qui ont étudié le rôle des interactions sociales entre pairs dans le développement de l'intelligence en référence au structuralisme de la théorie piagétienne. Ce courant regroupe les différentes études présentant les premières situations qui ont permis de valider cette conception à partir des tâches piagétiennes, comme la tâche de transformation spatiale et celle de conservation des longueurs (Doise et Mugny, 1981; Mugny (éd.) 1985; Perret-Clermont, 1979; Perret-Clermont (éd.), 1988). À travers différentes expériences, l'accent est mis sur les éléments et les conditions qui sous-tendent la production d'un conflit socio-cognitif (Carugati et Mugny, 1985; Doise et Mugny, 1981; Doise, 1985); sur le type de régulations engendrées par ce conflit socio-cognitif (Carugati, de Paolis et Mugny, 1981) et sur une forme de conflit socio-cognitif portant sur les significations de situations socialement marquées (Doise, 1988; Perret-Clermont, 1988).

Un deuxième courant théorique représenté par Gilly, Blaye et Roux (Gilly, 1988; Gilly, Blaye et Roux, 1988) met en évidence l'intérêt pour la construction de compétences limitées à des classes de problèmes et la référence à l'approche procédurale adoptée en résolution de problèmes. Ce courant théorique a donné lieu à un ensemble de travaux empiriques à propos du rôle des interactions de résolutions entre pairs (Blaye, 1986, 1988; Dalzon, 1988; Fraisse, 1985, 1987; Gilly, Fraisse et Roux, 1984; Gilly et Roux, 1988), du marquage social (Gilly et Roux, 1988; Roux et Gilly, 1984, 1988; Zhou, 1987, 1988) et de la recherche d'autres mécanismes de progrès cognitif (Gilly, 1988); de types d'intervention provoquant la déstabilisation dans des situations spécifiques de résolution et de l'identification de différents types de co-élaboration (Gilly, Fraisse et Roux, 1984).

Toujours selon le premier critère de classification de Gilly, dans une situation à caractère dissymétrique ou asymétrique, les chercheurs se regroupent sous trois orientations de recherche définies par le statut et les rôles assignés dans ce type de situation. Une première orientation se dessine en fonction du rôle de l'imitation dans le développement cognitif (Bandura, 1971, 1977; Emler et Glachan, 1985; Nadel, 1986 et Winnykamen, 1985, 1987, 1988). Une deuxième orientation se définit en rapport aux interactions de guidage ou de tutelle (Barnier, 1987; Wertsch, 1978, 1979, 1980; Wertsch et Stone, 1985). Enfin une troisième orientation se réalise grâce aux interactions de communication 
sociale (Beaudichon, 1985; Beaudichon et Vandesplas-Holper, 1985; Hennebelle, 1986; Robinson, Silbereisen et Claar, 1985).

Parallèlement à ces deux grands mouvements de recherche dont le rôle des interactions sociales demeurent une préoccupation centrale autant pour les adeptes de la psychogénétique que pour ceux s'inscrivant dans une approche procédurale de résolution de problèmes, un autre mouvement de recherche s'inscrit dans une préoccupation, certes, des interactions sociales dans le développement de l'intelligence mais en adoptant un cadre théorique autre que celui de la psychologie sociale génétique soit à partir d'aspects particuliers des interactions sociales identifiés par Piaget, Vygotsky, Wallon et d'autres.

\subsubsection{La recherche sur les interactions sociales}

L'intérêt croissant de l'aspect social dans le développement cognitif a généré la réalisation de diverses recherches sur le concept d'interaction sociale en identifiant des convergences sur les plans épistémologique et théorique et des convergences sur le plan méthodologique.

Sur le plan épistémologique, un bon nombre de recherches à partir des années 1980 adopte une position constructivisme-interactionnisme et socioconstructivisme-interactionnisme qui considère à la base fondamentale du développement, le sujet comme étant l'artisan et le responsable de la construction et de l'organisation des connaissances. L'agencement significatif de sa connaissance lui appartient en propre et celui-ci lui est unique. Cependant, les divergences apparaissant ici dans l'équilibration recherchée tout au long du développement de l'intelligence dans les différentes situations que vit le sujet serait l'oeuvre, dans une épistémologie constructiviste, de confrontations essentiellement individuelles faisant référence à une psychologie à deux termes -Ego-Objet- (Piaget, 1975) et, dans une épistémologie socio-constructivisme, de confrontations interindividuelles faisant référence à une psychologie à trois termes-Ego-Alter-Objet- 4 (Moscovici, 1972). Il est à noter que pour Moscovici,

4 Ego et Alter désignent, ici, des individus ou des groupes et objet, l'environnement social ou non social, réel ou symbolique (Marc et Pinard, 1989, p.5) 
Seule une telle psychologie mérite pour lui le qualificatif de sociale car elle est centrée directement sur l'élaboration et le fonctionnement du lien social et de son rôle fondamental, tant dans le fonctionnement psychique individuel que comme facteur d'infléchissement des phénomènes sociaux (Marc et Picard, 1989, p.15).

Selon ces auteurs, c'est dans ce sens que la notion d'interaction sociale doit être comprise.

Il ne s'agit donc pas d'opposer, par cette expression, des interactions qui seraient sociales à d'autres qui ne le seraient pas, mais de montrer la part du social présente dans toute rencontre; elle est repérable même dans les relations les plus intimes, cela vient du fait que toute rencontre interpersonnelle suppose des interactants ${ }^{5}$ socialement situés et caractérisés et se déroule dans un contexte social qui imprime sur elle sa marque en lui apportant un ensemble de codes, de normes et de modèles qui à la fois rendent la communication possible et en assurent la régulation (Marc et Picard, 1989 , p.15).

Mais il ne faudrait pas prendre cette marque comme un simple effet de détermination; il s'agit d'une relation dialectique. Car si l'interaction est le champ où les rapports sociaux actualisent et se produisent, elle constitue aussi un espace de jeu où peuvent s'introduire l'intervention et le changement et où, à chaque instant, se fonde à nouveau le lien social.

Son épistémologie se fonde sur le concept d'information [...] Ses principes sont d'ordre cybernétique, sa causalité de nature circulaire, rétroactive et l'information en constituant l'élément central, elle a pour objet les processus de communication à l'intérieur de systèmes au sens le plus large du terme (Waltzlawick, 1981, p.15: voir: Marc et Picard, 1989, p.11).

Sur le plan méthodologique, la convergence comporte certaines caractéristiques essentielles. Elle donne d'abord la primauté à une démarche d'observation et de description s'appuyant souvent sur des formes d'enregistrements (photos, magnétophones, vidéos, films). Elle privilégie une observation «naturaliste», un travail de terrain qui s'efforce de saisir et d'analyser des situations de la vie quotidienne, des interactions réelles et authentiques et non des situations provoquées et étudiées en laboratoire par le chercheur. Elle se centre sur les processus de communication considérés comme un phénomène global intégrant plusieurs modes de comportement (la parole, la mimique, le regard, les gestes, la distance interpersonnelle ...). Enfin, elle implique dans la construction de l'objet l'«abandon du sujet monadique au profit de l'interaction» (Cosnier, 1986, p.22: voir:

5 Le néologisme d'interactant désigne les sujets impliqués dans l'interaction. 
Marc et Picard, 1989, p.12). Il ne s'agit pas de comprendre et de théoriser le fonctionnement du sujet isolé, mais de considérer celui-ci comme un élément d'un système plus vaste qui inclut la relation à autrui et le contexte; c'est ce système qui constitue l'unité de base de l'analyse.

Ces différentes constatations et orientations de la recherche en psychologie sociale et sur les interactions sociales en général, nous amènent à circonscrire davantage le contexte dans lequel se réalisent les interactions sociales. Partant de la problématique de notre recherche, nous examinerons plus particulièrement la spécificité des interactions sociales influençant et déterminant, à la fois, l'activité de construction et l'activité de jeu symbolique.

\subsubsection{La recherche sur les interactions sociales et le jeu symbolique social}

Pour circonscrire l'ensemble des recherches, nous avons retenu les études sur les interactions sociales dans le contexte particulier du jeu symbolique, puisque le jeu symbolique a été identifié dans la problématique de notre étude comme étant une activité importante simultanée ou subordonnée à l'activité de construction suscitée par le matériel bloc.

Pour réaliser la synthèse des recherches sur les interactions sociales et le jeu symbolique social, certains critères tels que l'objet de recherche, les thèmes retenus, les années de publication et les cadres théoriques de référence ont orienté les grandes lignes de cette synthèse.

Trois temps importants ont marqué la recherche sur les interactions sociales et le jeu symbolique social.

Avant les années 80 , les interactions entre les enfants ont surtout été abordées dans une perspective théorique du développement cognitif de l'enfant en examinant les formes de participation sociale telles que définies et expliquées par Piaget. D'ailleurs, de nombreuses recherches sur les interactions des enfants ont été réalisées à partir de l'échelle de mesure de l'activité sociale élaborée par Parten (1933) en référence aux travaux de Piaget; cette échelle de mesure met en rapport des activités de jeux et des comportements sociaux 
spécifiques et respecte la conception hiérarchique de Piaget quant à l'évolution du comportement le plus individualisé jusqu'au plus hautement socialisé. Les comportements solitaires ou parallèles constituent les formes individuelles et les comportements associatifs et coopératifs représentent les formes collectives. Les efforts des chercheurs se sont orientés dans l'identification de catégories variées de participation sociale (Rubin, Fein et Vanderberg, 1984). Ces différentes catégories sont devenues un cadre de référence pour un bon nombre de recherches, en particulier pour celles préoccupées par le jeu symbolique social qui augmenterait avec l'âge et deviendrait plus organisé et coopératif (McLoyd, 1980; Rubin, Maioni et Hornung, 1976; Rubin, 1977, 1980).

À cette époque, les interactions sociales étaient envisagées comme l'aboutissement d'un long processus hiérarchique de socialisation. Dans ce processus, la coopération constituait, selon un point de vue piagétien, l'étape ultime de développement. Dans ce continuum de développement, les interactions sociales ne sont structurantes qu'au dernier niveau du développement, négligeant ainsi le rôle «moteur structurant» qu'elles peuvent jouer dans les autres étapes du processus de socialisation et référant ainsi un cadre d'analyse hautement individualiste (Garnier, 1987).

C'est dans cette vision développementale que Parten (1933) a élaboré des stades évolutifs des jeux des enfants au préscolaire, respectant à la fois une progression linéaire des activités ludiques et un développement de l'enfant passant progressivement de l'égocentrisme à une pensée socialisée et coopérative. L'enfant joue seul, en parallèle, en association avec les autres et enfin, en coopération. L'intérêt autour des interactions sociales entre enfants à l'intérieur de ces différents modes de jeux questionne peu ou pas l'ordre établi. Ainsi, les interactions sociales structurantes apparaissent qu'en bout de ligne de la hiérarchie de l'individuel ou social, ne jouant qu'un rôle bien secondaire dans les étapes subséquentes à la socialisation.

Au début des années 80 , les écrits de Vygotsky refont surface et accordent une place de toute autre importance à l'aspect social dans le développement cognitif de l'enfant. Cette perspective théorique entraine donc une interprétation et une appréhension nouvelle de l'interaction sociale et commence à marquer la recherche de ses nouvelles empreintes. 
Enfin, vers le milieu des années 80, l'élaboration de la théorie psychosociale génétique du développement cognitif (Doise, Mugny et Perret-Clermont, 1975) apporte un regard neuf et différent sur le rôle des interactions sociales dans le développement cognitif. Dans ce cadre d'interprétation psychosociogénétique, les interactions sociales sont envisagées comme des rudiments sociaux instituants et structurants des constructions cognitives dans les dynamiques individuelles plutôt que des indicateurs de socialisation rendue possible par une certaine maturité cognitive.

L'approche par catégorie, avant les années 80 , a donc été un des premiers cadres de référence pour étudier les formes de participation sociale générées par le jeu symbolique social. Par la suite, cette approche a ouvert des voies d'interrogation à d'autres préoccupations de recherche. Depuis le début des années 80 , l'emphase est mise sur la façon dont les enfants développent mutuellement les représentations significatives des objets, des événements et des rôles (Göncü, 1985, 1987a, 1987b; Göncü et Kessel, 1988; Wolf, Rygh et Altshuler, 1984).

La consultation des différents travaux de recherche, en ce qui concerne le concept d'interaction sociale et le jeu symbolique social depuis le début des années 80 , a conduit à l'analyse d'une quarantaine d'études. L'ensemble de ces études regroupe des enfants dont l'âge se situe entre quatorze mois et six ans. Elles ont abordé la problématique des interactions sociales et du jeu symbolique social en impliquant des enfants en dyades formées de multiples combinaisons: dyade d'enfants du même âge, du même sexe et de sexe différent; dyade mère-enfant; dyade d'enfant plus jeune et d'enfant plus vieux; dyade d'enfants dits normaux avec un enfant handicapé au niveau du langage, des enfants sourds, des enfants autistiques; dyade des novices et des experts: un novice et un expert).

Quatre études ont été réalisées avec des enfants regroupés en triades; là aussi avec des combinaisons diverses: deux enfants amis et l'enfant familier, deux enfants amis avec l'enfant négligé, rejeté et controversé. Dans ce cas, les statuts sociaux des enfants ont été déterminés selon des mesures sociométriques établies a priori de l'expérimentation.

Les objets de ces recherches convergent tous vers une meilleure compréhension de l'interaction sociale qui rend possible la «réussite» du jeu symbolique social. 
Dans le jeu collectif, la signification des objets et des actions ainsi que le thème et son déroulement doivent être partagés par les partenaires, puisque la «réussite» du jeu de fiction collectif tient au fait qu'on peut le construire ensemble, qu'il est cohérent et qu'il dure. Ceci dépend de l'équilibre entre les intentions ou les idées du partenaire et celle de l'autre ou des autres. (Stamback et al., 1990, p.14)

Dans la littérature, le concept d'interaction sociale apparaît d'abord comme un processus de communication (Marc et Picard, 1989, p.19). «Dans la plupart des situations, la relation entre deux ou plusieurs individus se traduit par une communication et notamment par un échange de paroles.». L'ensemble des études sur les interactions sociales et le jeu symbolique social ont abordé sous différents angles ce processus de communication.

La communication apparaît comme un phénomène relationnel ou les interactants, la situation, les comportements interagissent étroitement entre eux, formant un système circulaire d'actions et de réactions, de stimulus et de réponses. Ce système a sa propre dynamique et ses propres régulations dont aucun des interactants n'a la maîtrise complète. Même si chacun intervient à partir de motivations et d'enjeux personnels, la communication est nécessairement de nature transactionnelle et les stratégies qu'elle entraîne de type interactif. (Marc et Picard, 1989, p.20).

Les chercheurs de la psychologie évaluative incorporent des acquis des théories communicationnelles pour envisager le jeu symbolique social et les interactions sociales en termes de processus évolutif.

En ces termes, le jeu symbolique social dévoilerait la recherche et l'atteinte du consensus parmi les partenaires de jeu et serait considéré comme un ensemble de composantes actives et organisées dans le temps. De plus, les analyses dynamiques de ce processus révéleraient plusieurs formes possibles de jeu social dépendantes du degré et du type de consensus parmi les joueurs. Le jeu symbolique social a donc été identifié par Göncü (1987b) comme un processus de négociation fournissant aux enfants une tentative d'atteindre des accords minimaux afin de maintenir le jeu.

En termes de processus évolutifs, les interactions sociales sont examinées dans un but de compréhension de la qualité de l'interaction, de l'identification et de l'évolution des différents procédés et stratégies permettant une construction collaborative et coopérative entre les partenaires de jeu. 
Ainsi, l'appréhension de l'objet de recherche lui-même, l'interaction sociale et le jeu symbolique tentent progressivement de mettre en évidence un regard qualitatif sur les observations, en se préoccupant davantage des processus, des procédés et des stratégies interactifs des enfants dans le jeu symbolique en requestionnant et repositionnant les idées de Piaget sur les interactions sociales ou en utilisant des conceptions psychosociales du développement cognitif (Ballion et al., 1990; Sinclair et al., 1982; Stamback et al., 1983).

C'est donc à travers l'étude de ce processus de communication se traduisant par les énoncés verbaux des enfants, la communication non verbale, les gestes et les actions des enfants, sur les objets et vers les autres personnes que les chercheurs ont tenté d'identifier et de définir les différents processus, procédés, stratégies qui permettent de jouer à plusieurs (Ballion, Breaute, Rayna et Stamback, 1987; Black, 1989, 1992; Black et Hazen, 1990; Farver, 1992; Göncü, 1985; Göncü et Kessel, 1988; Howes, 1988, 1993; Howes, Droege et Matheson, 1994; Kane et Furth, 1993; Verba, 1990).

Dans cette voie de recherche, sur le plan théorique, l'interaction sociale et le jeu symbolique social sont abordés selon des conceptions constructivistes-interactionnistes et socio-constructivistes-interactionnistes qui apportent des éclairages nouveaux et différents pour comprendre la dynamique sociale dans la construction des savoirs.

Sur le plan méthodologique, certains chercheurs abordent maintenant l'étape de la méthodologie observationnelle d'une manière beaucoup plus qualitative, en tenant compte de la complexité de tous les paramètres qui permettent d'identifier les composantes spécifiques de l'observation dans la cueillette des données. On parle davantage dans ce cas de l'observation expérientielle que d'observation systématique (DeKetele et Postic, 1988).

Dans l'ensemble des recherches examinées, un écart encore bien substantiel se situe entre le dépouillement des données et l'analyse de ces données. Toutes les recherches rendent compte d'une transcription assez raffinée des énoncés verbaux et non verbaux, des actions, des gestes et des objets utilisés, mais qui finissent par se regrouper dans des catégories déterminées a priori sans nécessairement tenir compte des éléments émergents et particuliers qui s'en dégagent. 
On perçoit un mouvement d'analyse qualitative des données, mouvement qui semble embrasser la complexité des situations observées et qui est beaucoup plus présent dans la recherche. Mais on revient encore assez rapidement à une analyse conservatrice et traditionnelle en continuant d'utiliser l'analyse statistique pour le traitement des données obtenues et la description linéaire de ces résultats. Il est important de souligner que les analyses de nature plus qualitative et ses résultats n'en sont encore qu'à leur premier balbutiement et que les changements d'analyse et d'interprétation dans les pratiques de recherche, comme dans tout autre domaine, sont lents et se heurtent d'abord à des résistances avant de faire un consensus dans la communauté scientifique.

Cette démarche d'exploitation conceptuelle et théorique des domaines d'investigation du jeu symbolique et des interactions sociales nous conduit à élaborer les questions de recherche inhérentes à notre problématique.

\subsubsection{Vers la formulation des questions de recherche relatives à l'activité de l'enfant avec le matériel bloc}

Considérant les deux questions générales de recherche: Quelle est la nature des activités que le matériel bloc suscite chez l'enfant? Et est-ce que ce matériel et les activités qu'il suscite contribuent au développement cognitif et social de l'enfant qui fréquente la classe maternelle?, la démarche réalisée jusqu'à maintenant afin d'élaborer la problématique et d'identifier les différents éléments théoriques et conceptuels, nous amène à formuler nos questions de recherche selon trois enchaînements successifs qui témoignent de cette dynamique que l'on souhaite décrire et comprendre.

Au départ, la démarche de recherche a engendré deux types de questionnement. Un premier questionnement sur les potentialités symboliques du matériel, sur le contexte environnemental de l'organisation matérielle de cette aire d'activité dans la classe maternelle et un deuxième questionnement, sur l'examen de l'activité de l'enfant dans cette aire de jeu avec le matériel bloc par son observation, sa description et son analyse.

Centrée sur l'activité de l'enfant suscitée par le matériel bloc, cette étude veut mettre en évidence les potentialités du matériel, les composantes et les produits de cette activité 
afin d'identifier certains paramètres particuliers quant à la nature et la structure de cette activité.

Du premier enchaînement, dans l'identification de paramètres plus particuliers, il nous semble que la nature de cette activité peut être qualifiée de symbolique, due au mode symbolique d'appréhension du monde caractérisant l'enfant de cinq ans. De plus, cette nature symbolique est issue, d'une part, du matériel lui-même, les blocs et, d'autre part, de l'activité de l'enfant avec ce matériel qui s'actualise selon deux formes de représentation.

La première forme de représentation sous-tend notre première question spécifique de recherche et se manifeste d'abord dans l'activité de construction proprement dite en faisant référence aux composantes et aux produits mis en branle pour rendre possible l'activité de construction. Dans son activité de construction, l'enfant, à partir des blocs, crée des objets et des événements. Faisant référence aux écrits de la perspective psychologique et d'une approche développementale, Johnson (1974) a identifié certains procédés de constructions correspondant à des stades ou étapes d'évolution de l'activité de construction, d'où la formulation de la première question spécifique de recherche:

\section{À quel stade ou à quelle étape d'évolution de l'activité de construc- tion se situent les enfants de cinq-six ans qui fréquentent la classe mater- nelle?}

Le deuxième enchaînement s'inscrit dans la deuxième forme de représentation de l'activité symbolique, celle qui se manifeste par l'activité de jeu symbolique. En effet, l'activité de construction génère différentes productions qui invitent à des jeux symboliques.

Toujours en référence aux éléments théoriques retenus de l'approche développementale dans une perspective psychologique, plusieurs auteurs, à la suite de Johnson, ont identifié, raffiné et complété les stades d'évolution de l'activité de construction. Un point de convergence fait consensus pour l'ensemble des auteurs, vers cinq-six ans, ils observent le stade du jeu dramatique. En effet, on voit apparaître une forte impulsion au jeu dramatique à partir et autour des productions de l'activité de construction. Les blocs de- 
viennent un cadre de référence dans lequel s'élabore le jeu dramatique qui deviendrait coopératif et interrelié entre les enfants. Cependant, même si tous ont observé le stade du jeu dramatique, aucun d'entre eux n'apporte d'éléments d'observation, de description et d'analyse sur la spécificité de ce jeu dramatique élaboré à partir du matériel bloc. D'où la formulation de la deuxième question spécifique de recherche:

Selon les stades d'évolution de la construction, les enfants de la maternelle se situeraient au stade du jeu dramatique:

\section{Quelles sont les composantes structurales de l'activité de jeu symbo- lique à partir du matériel bloc?}

Enfin le troisième enchaînement s'insère dans la réflexion des interactions sociales qui sous-tendent l'activité de construction et l'activité de jeu symbolique.

Dans le contexte de la classe maternelle et de l'espace réservé au matériel bloc, l'activité de construction et l'activité de jeu symbolique suscitées par ce matériel sont de plus en plus réalisées à deux, trois et quatre enfants. Donc ces activités s'organisent en situation de collaboration entre les partenaires.

De ce fait, il nous apparaît qu'un ensemble d'éléments essentiels dans la structure de l'activité de l'enfant avec le matériel bloc se situe sur le plan interactionnel, c'est-à-dire sur le plan des interactions sociales entre les enfants. Ces mêmes éléments influenceraient et détermineraient l'activité de construction et l'activité de jeu symbolique simultanée ou subordonnée à l'activité de construction.

En posant la prémisse que les interactions sociales jouent un rôle dans le développement cognitif et social de l'enfant, on peut se demander:

Quels types d'interactions sociales émergent de l'activité de l'enfant avec le matériel bloc?

Dans un premier mouvement, nous nous attendons pertinemment à ce que les enfants qui choisissent le coin bloc s'adonnent à une activité dite de construction. Cette ac- 
tivité peut alors, dans ce cas-ci, se réaliser à deux, trois ou quatre enfants et déjà, selon l'agencement des enfants, cette première activité crée un réseau d'interactions afin de favoriser la réalisation d'un projet de construction collectif. Déjà, il est légitime de se demander:

Quelles sont les interactions sociales qui émergent de la dynamique de l'activité de construction au coin bloc? Quelles sont celles qui favorisent davantage la réalisation d'un projet collectif de construction? Contribuent-elles au développement cognitif et social de l'enfant?

Dans un deuxième mouvement, l'observation des enfants au coin bloc et la revue des écrits nous rappellent que l'enfant de 5-6 ans qui fréquente la classe maternelle se situe dans une période de son développement particulièrement féconde sur le plan symbolique. À cet âge, il appréhende le monde qui l'entoure par un mode symbolique qui se traduit fortement dans son activité ludique. Ce mode symbolique d'appréhension du monde alimente et teinte la majorité des actions et des interactions des enfants. Il devient en quelque sorte la trame de fond sur laquelle se tisse le mode de développement de l'enfant de 5-6 ans, et par conséquent, la manifestation du jeu symbolique s'observe aisément dans les différentes activités de la classe maternelle et, plus spécifiquement dans l'activité de l'enfant au coin bloc. Donc, on peut ici encore une fois s'interroger et se demander:

Quelles sont les interactions sociales qui émergent de la dynamique de l'activité de jeu symbolique au coin bloc? Quelles sont celles qui favorisent davantage la réalisation d'un projet collectif de jeu symbolique? Contribuent-elles au développement cognitif et social de l'enfant?

Ces trois enchainements successifs identifiés dans la formulation des trois questions spécifiques de recherche nous amènent à formuler les objectifs de cette étude:

Dans un premier temps, OBSERVER l'activité de l'enfant suscitée par le matériel bloc. De ces observations, DÉCRIRE et ANALYSER les caractéristiques propres de l'activité de construction selon les stades d'évolution de la construction. DÉGAGER les composantes et les produits qui en découlent. 
Dans un deuxième temps, OBSERVER l'activité de jeu symbolique qui s'élabore sous un mode simultané ou subordonné à l'activité de construction. De ces observations, DÉCRIRE et ANALYSER les différentes composantes structurales de l'activité de jeu symbolique générée par un matériel particulier soit les blocs. IDENTIFIER les spécificités de cette activité reliées à la nature même du médium utilisé.

Dans un troisième temps, OBSERVER le type d'interactions sociales qui émergent, d'une part, de l'activité de construction et, d'autre part, de l'activité de jeu symbolique. De ces observations, DÉCRIRE et ANALYSER les interactions sociales qui favorisent davantage la réalisation d'un projet de construction et d'un projet de jeu symbolique. IDENTIFIER les particularités des interactions sociales communes et spécifiques à chacune des activités et leurs contributions potentielles au développement cognitif et social de l'enfant. 


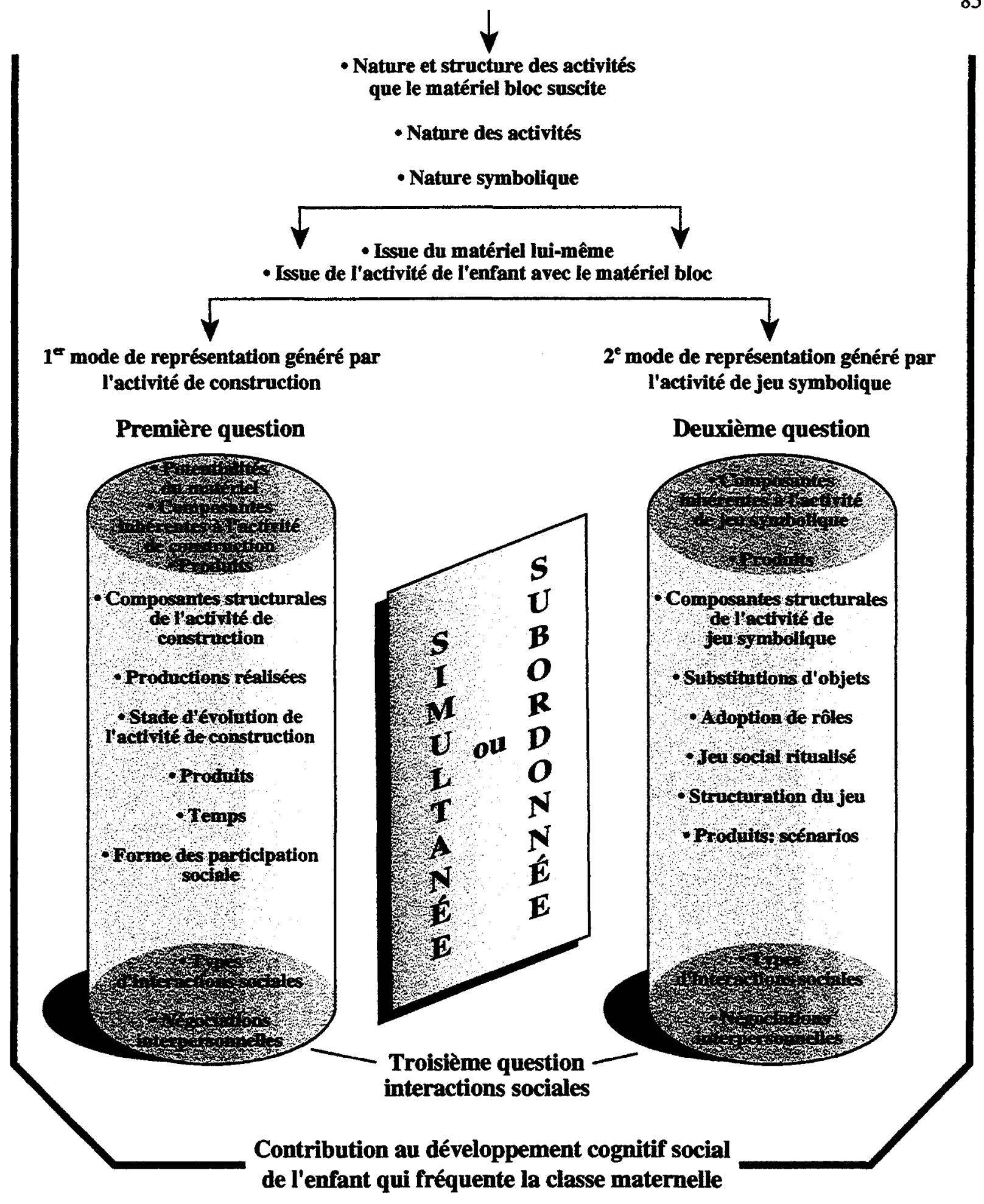

Figure 2.1

Synthèse relative à l'interrelation des trois questions de recherche de l'activité de l'enfant suscitée par le matériel bloc 


\section{CHAPITRE III}

\section{D'UNE PROBLÉMATIQUE DE RECHERCHE SUR L'ACTIVITÉ DE L'ENFANT AVEC LE MATÉRIEL BLOC \\ À SON OPÉRATIONNALISATION: L'APPROCHE OBSERVATIONNELLE}

\subsection{L'observation comme démarche privilégiée}

L'observation, comme technique de cueillette de données, peut s'inscrire aussi bien dans une approche positiviste (quantitative) que compréhensive (qualitative) de recherche.

Cependant, le concept de l'observation prendra une coloration particulière dépendant de l'approche privilégiée, et il est bien certain qu'un grand nombre de concepts qui se greffent à l'observation prendront eux aussi un nouveau sens. Ainsi les concepts de science, faits, lois, théories, validité et critères de scientificité se définiront en harmonie avec l'approche préconisée.

Considérant l'option constructiviste et socio-constructiviste comme cadre conceptuel et considérant les choix épistémologiques eu égard à la conception du développement de la connaissance, du développement de l'enfant, du jeu et du jeu symbolique, cette étude s'inscrit donc dans une recherche qualitative. Cette approche permet l'étude du phénomène au-delà des visions traditionnelles. Elle permet d'enrichir par de nouveaux éléments la compréhension des phénomènes à l'étude.

Le choix d'un cadre conceptuel constructiviste et socio-constructiviste pose le problème du développement de la connaissance à partir d'une vision tripolaire, incluant la 
vision bipolaire piagétienne du sujet et de son interaction avec l'objet, en accordant une place privilégiée à autrui dans le mouvement de l'interaction de l'autre dans l'appréhension de l'objet et dans l'interaction de l'environnement humain et physique au soi. Ce sont évidemment ces mêmes éléments qui doivent être pris en compte dans le cadre de l'observation.

L'observation est considérée comme motivée, contingente de nos attentes conceptuelles ou autres. Elle comporte toujours un choix conditionné par les conceptions théoriques ou idéologiques du chercheur et déterminé par les finalités de l'expérience. L'observation est donc considérée comme un processus actif au cours duquel la connaissance est construite par un sujet connaissant et non par l'attitude passive d'un sujet qui enregistre les données de la réalité. (Anadón, 1988, p. 9).

Cohérente avec ce choix épistémologique, la méthodologie s'inscrit dans une approche qualitative, où l'observateur est défini comme étant une personne qui observe avec, d'une part, ses croyances, ses valeurs et ce qu'il est et, d'autre part, avec un contexte théorique qui lui permet de construire l'objet qu'il observe. Déjà, l'observation est orientée par des éléments théoriques et idéologiques, des croyances, des valeurs et autres qui ont permis de déterminer les différentes dimensions à observer. De plus et surtout, l'observation dans ce cadre tient compte des éléments qui peuvent émerger de la situation à l'étude. Ainsi, au fur et à mesure, les éléments qui émergent de la situation viennent raffiner peu à peu les dimensions à observer.

Avant d'aborder les éléments constitutifs de cette étude au plan méthodologique, il apparaît important de situer le choix de cette méthodologie dans une réflexion plus globale de la recherche, l'approche qualitative. Les liens qui se tissent dans la démarche même de la recherche à chacune des étapes (surtout non linéaires) soit la délimitation de la problématique de recherche, la formulation des questions relatives à cette problématique, l'élaboration d'un cadre conceptuel permettant de filtrer la dynamique privilégiée et le mode d'investigation préconisé s'inscrivent, selon, dans un modèle de compréhension.

\subsection{Les fondements de l'observation}

La méthodologie est une phase importante de la recherche, elle correspond, selon Lafortune (1989), au développement concret de la recherche dans son ensemble. Cette 
phase de la recherche permet la réflexion sur la manière la plus significative et pertinente de puiser l'information nécessaire à la compréhension du phénomène choisi.

La recherche scientifique s'élabore autour de trois dimensions du savoir: la dimension épistémologique, la dimension théorique et la dimension technique. Ces trois dimensions sont en interrelation et interrogent mutuellement chaque concept qui se greffe à l'objet de recherche considéré. (Goyette, 1988)

De plus, un design de recherche doit prendre en compte ces trois aspects de la recherche et doit en plus expliciter les rapports qui lient chacune des dimensions.

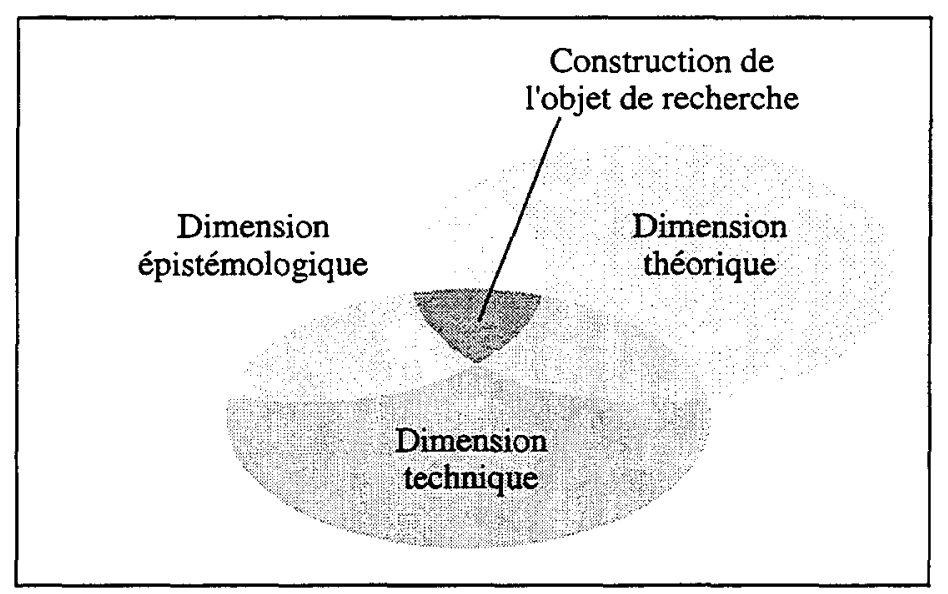

Figure 3.1 Vision intégrative des trois dimensions du savoir

Dans une vision intégrative des trois dimensions du savoir, la dimension épistémologique, comme composante d'une démarche de recherche, consiste à jeter un regard critique autant sur les modes de production de connaissances que sur les procédures de validation. Dans une recherche particulière, les questions épistémologiques se situent souvent au niveau des postulats implicites et explicites. Dans le cadre de cette étude, nos présupposés théoriques trouvent leurs fondements dans une approche constructiviste et socioconstructiviste du développement de la connaissance et ce, dans une épistémologie qualifiée de sociale et génétique.

Dans la même ligne de pensée, la dimension théorique s'intéresse à une théorie qui est un système conceptuel mettant en relation un ensemble de phénomènes sous observation et un système de représentation de ces phénomènes à partir de postulats explicites. 
Dans une recherche particulière, les questions théoriques se situent au niveau du choix d'une ou de théories dont les postulats de base s'inscrivent dans une compréhension du monde identifiée par la réflexion épistémologique. Dans la présente recherche, nous avons retenu des éléments de la théorie piagétienne, de la théorie vygotskienne et de la théorie intégrative des aspects individuel et social du développement cognitif, soit la psychologie sociale génétique.

Enfin, dans sa dimension technique, la recherche a pour objectif de comprendre la réalité. Cette réalité doit être observée au moyen de techniques spécifiques (entrevues, observation participante, questionnaires, etc.). Ce sont ces techniques et leurs modalités d'utilisation qui distinguent l'activité scientifique d'une activité d'investigation ordinaire.

La transformation de la réalité en données de recherche se fait à travers le système de représentation de cette réalité utilisée par tout chercheur. Il s'établit alors une sélection qui agit à plusieurs niveaux.

Dans l'élaboration de la méthodologie, la dimension technique doit tenir compte du lien technique-épistémologique et technique-théorique. Il faut donc prendre en compte les dimensions épistémologique et théorique, puisque la façon de poser le problème de la connaissance est à l'origine même de la façon d'envisager les moyens techniques à utiliser pour recueillir des informations adéquates et pertinentes à l'objet de recherche. C'est pourquoi il est important de considérer ces rapports pour déceler la cohérence interne de la recherche.

La tradition de recherche a souvent été le critère de choix de la méthodologie quels que soient les objets de recherche, les fondements théoriques et les domaines d'étude. La méthode expérimentale étant la seule qui pouvait guider les procédures à mettre en branle pour aboutir et produire des connaissances qualifiées de scientifiques.

Aujourd'hui, les questionnements relatifs aux objets de recherche dans les différents domaines et les questionnements soulevés par les modes de production de connaissances amènent les chercheurs à s'interroger sur les fondements de la science et sur les processus de production de connaissances. 
Considérant l'objet de la présente recherche, soit l'étude de l'activité de l'enfant avec le matériel bloc et considérant que cette étude veut mettre en évidence la nature, la structure, les composantes et les produits de l'activité de construction et de l'activité de jeu symbolique, il semble que l'observation dans une perspective méthodologique qualitative est adéquate et pertinente pour la cueillette d'informations.

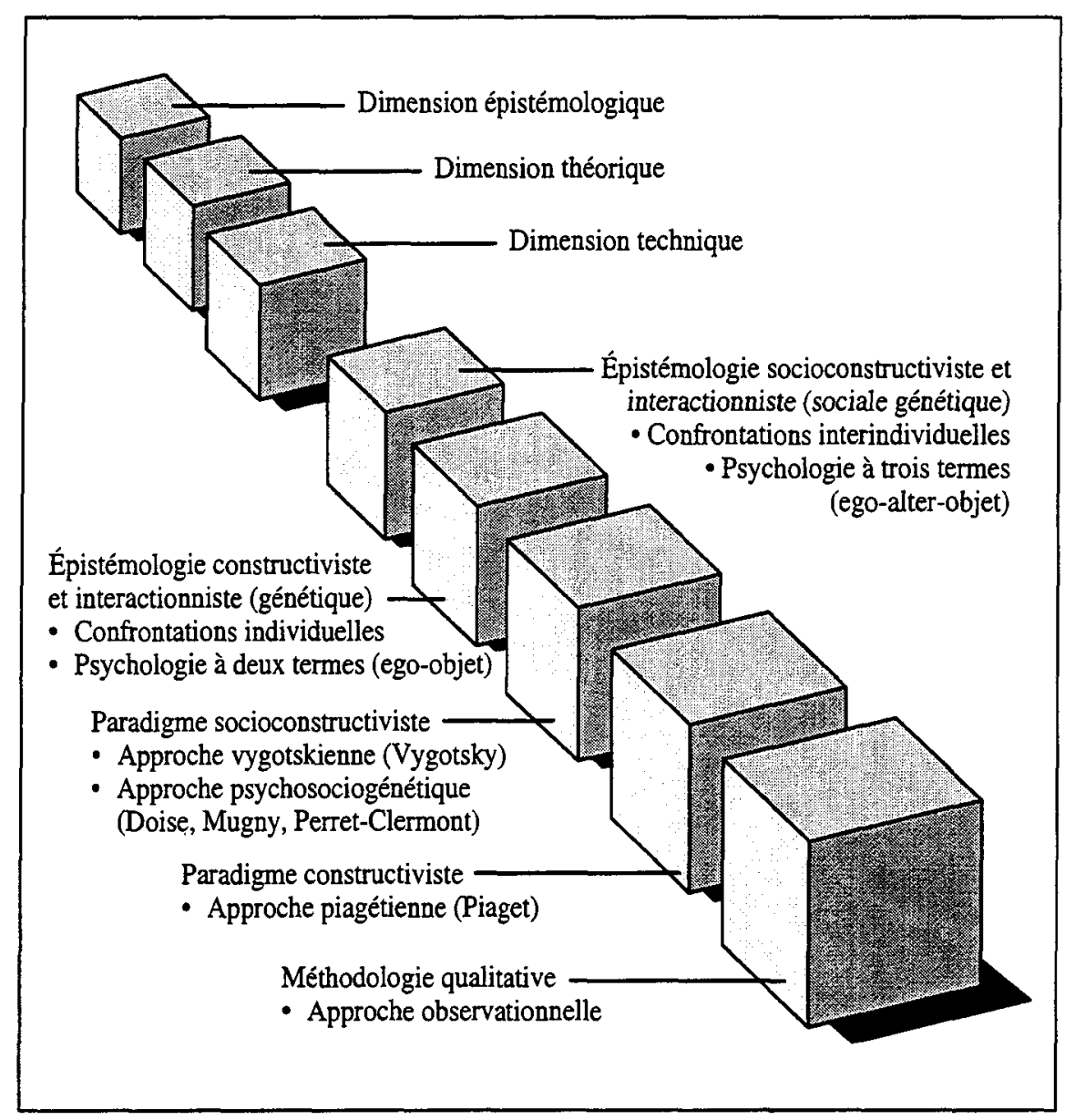

Figure 3.2 Vision intégrative des trois dimensions du savoir spécifique à notre étude

Cette approche observationnelle varie en fonction de l'approche de recherche, du type de science en cours d'élaboration, des champs de recherche et des utilisateurs de recherche.

Selon ce cadre de référence, l'observation utilisée dans une approche méthodologique qualitative pose des jalons différents de celle utilisée dans l'approche positiviste 
quant aux présupposés épistémologiques et théoriques. Ainsi, dans une méthodologie qualitative, les éléments constituant le cadre de l'observation, soit l'observateur, la situation observée et les acteurs en jeu, se trouvent modifiés et les différents concepts reliés à l'observation possèdent dès lors des significations différentes, puisque le cadre de référence est lui aussi différent.

Dans ce cadre, l'observation ne correspond pas à un codage d'éléments qui viennent se placer dans une grille préétablie. Elle correspond plutôt à une description, orientée par l'objet de recherche, de ce qui se passe dans la situation observée afin de mettre en évidence autant les particularités propres à l'objet d'étude que les traits communs. L'observation dans ce contexte est plus susceptible de saisir les mouvements dynamiques dans leur entité et leur complexité.

De Ketele et Postic (1988) distinguent deux types d'observation: l'observation systématique et l'observation expérientielle. La première relève du courant de la pédagogie expérimentale classique et son but vise à élaborer une catégorie et à décrire ce qui se passe dans la situation. Pour sa part, l'observation expérientielle est une investigation menée à partir de l'expérience d'une situation, vécue au fil du temps, par soi-même et par d'autres personnes. Elle favorise l'utilisation du modèle à prédominance clinique: les méthodes de l'observation clinique, l'observation naturaliste et ethnologique et l'observation participante. Une différenciation de ces deux types d'observation s'établit à partir de modalités de prélèvements des données.

Ces auteurs identifient neuf paramètres dans une typologie des modalités d'observation. Ces paramètres sont les fonctions, l'observateur, l'objet, les instruments, le degré d'inférence et le degré de liberté de l'observation. Ces paramètres permettent d'identifier les composantes spécifiques de l'observation dans d'étude de l'activité de l'enfant avec le matériel bloc.

La fonction de l'observation est descriptive et heuristique. Elle vise ici à décrire et à comprendre la nature, la structure, les composantes et les produits de l'activité de l'enfant avec le matériel bloc. De plus, elle est orientée vers l'émergence de dimensions qui vise à donner un sens aux informations recueillies pas à pas. 
L'observateur, en l'occurrence l'auteure de cette étude, est une observatrice participante passive que Mead (1966) explique comme étant:

l'observateur participant entre dans le jeu, observe, mais ne touche à rien. Il prend la peine d'étudier les mœurs des êtres humains auxquels il se mêle, jusque dans les plus infinis détails, et il se fait un scrupule de les laisser intactes, considérant le tissu de cette vie comme un apport précieux pour la science. (Mead, 1966: voir De Ketele et Postic, 1988)

L'observation porte sur des événements (des faits, au sens large) puisqu'elle oriente son attention sur des caractéristiques de la situation, sur des comportements et des interactions entre les personnes. L'observateur regarde l'action de l'enfant avec le matériel bloc; il observe ce qui se passe entre les quatre enfants et cherche à décrire la nature, les composantes et les produits de l'activité de construction et de jeu symbolique. De plus, l'enregistrement de l'observation est narratif et son attention porte sur le déroulement des actions, les effets des actions et sur les conséquences ultérieures de ces actions pour le déroulement de l'activité de construction et de jeu symbolique. Enfin, elle est allospective puisque l'observation de la situation est menée par l'auteure de l'étude et que celle-ci n'est pas impliquée dans la situation.

Les instruments d'observation permettent d'identifier une technique principale et dominante, soit celle de l'observation expérientielle. Cette technique narrative est plus ouverte et moins prédéterminée. L'instrumentation de l'observation expérientielle fait référence à un ensemble d'outils pour recueillir des données, directement ou indirectement adapté au cadre conceptuel et aux questions de recherche (Huberman et Miles, 1991). Dans le cadre de cette étude, des entrevues avec des enseignantes et des enfants, des observations d'enfants au coin bloc avec comme support technique un caméscope et des magnétophones, une liste des objets du coin bloc, une documentation du fonctionnement de la classe maternelle en général et du coin bloc en particulier, des photographies des productions des enfants et un journal de bord constituent l'ensemble des outils retenus.

Le degré d'inférence de l'observation est élevé puisque l'observateur énonce soit des intentions, soit des motifs, soit des sentiments; en un mot, il attribue une signification à ce qu'il observe. Ce processus d'inférence se fonde sur les effets du comportement, les relations entre les antécédents et conséquents et sur la propre expérience personnelle. 
La notation de l'observation est faite à partir de deux sources matérielles, vidéo et audio, à l'aide d'un caméscope et d'un magnétophone. Deux microphones, un relié au caméscope et l'autre au magnétophone, permettent de recueillir le discours des enfants en situation de jeu. Ainsi l'observation est médiatisée et plusieurs avantages lui sont habituellement reconnus.

La situation d'observation est faite en milieu naturel dans la classe maternelle, dans l'espace réservé au matériel bloc et elle sera, selon le déroulement de la recherche, manipulée ou non manipulée.

Le traitement des observations s'appuie sur des unités d'observation molaires qui forment directement une unité de signification et situées dans l'espace et dans le temps. Le traitement des observations repose sur un processus de recherche de sens. Quelques mécanismes de quantification seront utilisés pour certains aspects plus descriptifs. Dans le premier cas, on recourra à des processus plus ou moins intuitifs de recherche de sens (investigation des intentions, analyse des recoupements, recherche d'une structure, etc.). Dans le deuxième cas, on recourra à des techniques simples de comptage (analyse statistique descriptive). Enfin, le traitement sera séquentiel et qualitatif. Il y aura également chevauchement des deux types d'observation systématique et expérientielle, et ce, dans le recueil et l'analyse des données. Cependant, la cueillette et l'analyse de ces données seront essentiellement à prédominance qualitative.

\subsection{La planification de la cueillette des données}

La décision de l'échantillonnage s'est effectuée à partir de deux modes d'appropriation de cueillettes de données issus, d'une part, d'un cadre d'observation systématique et, d'autre part, d'un cadre d'observation expérientielle.

\subsubsection{La cueillette des données: l'observation systématique}

L'observation du comportement selon une approche éthologique, approche descriptive des sciences biologiques, apporte des pistes de réflexions intéressantes quant à la première étape de recherche. Cette approche se caractérise à la fois par l'utilisation systématique de l'observation directe du comportement et par une conceptualisation biolo- 
gique des activités humaines. Selon Strayer et Gauthier (1982), cette approche se découpe en trois phases distinctes et successives: l'analyse descriptive, la recherche exploratoire et l'évaluation systématique.

Dans la présente étude, la première phase de l'observation selon une approche éthologique est retenue. L'analyse descriptive vise essentiellement à décrire le plus exhaustif possible l'objet étudié et à en classifier les caractéristiques. Cette phase comporte trois étapes successives: l'observation naïve, l'élaboration d'une liste d'éléments et leur organisation.

L'observation naivve consiste en une observation informelle, où l'observateur identifie et décrit le comportement de ou des individus à l'étude. Le but visé est d'entrer en contact avec les sujets et leur milieu. Les périodes d'observation ne sont pas structurées et se distribuent tout au long du cycle d'activités des individus observés. Cette première étape permet de décrire le contexte physique du lieu d'observation, le contexte social et les schèmes comportementaux; de raffiner et de compléter les diverses connaissances déjà acquises du milieu des classes maternelles. Nous avons donc réalisé une tournée dans six classes maternelles, observé plus d'une centaine d'enfants à l'œuvre dans le coin bloc et échangé avec les six enseignantes de ces groupes d'enfants.

Sensible à l'importance du contexte physique, nous avons observé divers aménagements physiques de la classe maternelle qui sont souvent tributaires des dimensions de local-classe; différents aménagements du coin bloc dépendant de l'espace alloué pour cette aire de jeu et l'importance et de l'intérêt accordés aux activités dans cet espace. De plus, dans certaines aires, seul un matériel de blocs en bois vernis peut se retrouver tandis que dans d'autres coins, une variété de blocs au matériau, forme et design différents s'y retrouvent. Dans plusieurs classes, un matériel complémentaire accompagne les blocs, tels que figurines de personnages et d'animaux, arbres, fleurs, voitures, autobus, etc. Dans les différentes classes, chacune des enseignantes propose un type de rangement du matériel qui peut être à la fois original et efficace.

L'espace alloué et l'intérêt manifesté et accordé au coin bloc en classe maternelle s'inscrivent dans un fonctionnement général de la classe qui sous-tend une philosophie particulière de la pédagogie au préscolaire. Cette philosophie prend sa source dans les di- 
vers documents proposés par le ministère de l'Éducation (MEQ, 1981, 1982) et est opérationnalisée selon la compréhension, l'interprétation et le réinvestissement de chacune des enseignantes.

Habituellement, l'accès au coin bloc s'inscrit dans un fonctionnement par ateliers et ce choix est possible pendant l'heure consacrée au travail en atelier. Il arrive aussi que les enfants jouent aux blocs pendant la période qualifiée de jeux libres. Ce coin est réglementé de diverses façons. C'est souvent un espace contingenté, de façon provisoire et séquentielle. Dans certains cas, il ne sera accessible que du début de l'année scolaire jusqu'en novembre et dans d'autres cas, les enfants n'y joueront qu'à partir de décembre. Par contre, d'autres classes offrent ce choix quelques mois à l'automne et quelques mois au printemps. Les raisons de ces diverses réglementations demeurent toutefois très arbitraires et souvent sans fondements explicites. Le nombre d'enfants présents est lui aussi réglementé selon l'espace alloué, variant d'un minimum de deux enfants à la fois jusqu'à un nombre illimité, mais dans plusieurs cas, on retrouve une moyenne de quatre enfants.

Finalement, dans chacune des observations naïves, nous nous sommes sensibilisée aux diverses activités réalisées au coin bloc à la maternelle: des activités de construction à $1,2,3$, ou 4 enfants; des activités de jeu symbolique à également 1,2,3, ou 4 enfants; des activités de jeu symbolique simultanées ou subordonnées à l'activité de construction; l'exploitation de thèmes variés, la mise en place de scénarios de peu élaborés à complexes, l'apprentissage de divers rôles parentaux et sociaux; la planification de projets solitaires ou collectifs; la planification de scénarios; les interactions des enfants pour la réalisation des activités, pour la bonne marche des activités, pour des prises de décisions, pour des négociations sur des désaccords, pour partager des idées, pour soumettre des projets, etc.

La première sensibilisation aux différentes activités des enfants au coin bloc dans la classe maternelle a permis d'élaborer une liste descriptive qui rend compte le plus possible des activités des enfants avec ce matériel. Cette liste s'est raffinée progressivement au cours de l'observation naïve, afin de saisir le plus adéquatement possible l'ensemble des divers comportements liés aux activités des enfants avec le matériel bloc. Déjà, l'élaboration de cette liste descriptive permet de réaliser un regroupement d'éléments en catégories ou en classes. Cette liste, issue de l'observation naïve, a donc permis une première 
ébauche de ce que seront les grandes dimensions de l'observation et par la suite, de l'analyse des données ainsi recueillies.

\subsubsection{La cueillette des données: l'observation expérientielle}

Les données qualitatives sont caractérisées aussi par leur nature descriptive. L'observation est centrée sur le déroulement des activités de l'aire de groupe: observation intensive avec un œil ouvert, un esprit ouvert dans l'investigation (Gronbach, 1975, voir: De Ketele et Postic, 1988). Dans ce sens, les séquences d'événements sont enregistrées aussi fidèlement que possible.

Ce type de cueillette de données se distingue par le fait que la collecte des données est parallèle à l'analyse des données.

Si, au début, $80 \%$ du temps et d'effort se passent à recueillir les données, tandis que $20 \%$ sont consacrés à l'analyse, c'est l'inverse qui se produit vers la fin. Le modèle émerge progressivement et n'est jamais complet avant que l'investigation soit terminée. Partir avec des questions d'un large champ, rassembler les données qui y répondent, voir ce qu'elles signifient au moyen d'un cadre conceptuel, prendre des décisions au sujet de ce qui est à collecter, etc. (Owens, 1982: voir Huberman et Miles, 1991)

Différentes techniques d'observation expérientielle ont été identifiées pour cette étude. Parallèlement, à chaque séance d'observation enregistrée sur bande magnétoscopique, un journal de bord a été constitué dans lequel les grandes lignes de chaque séance d'observation et les faits déroulés avant et après la séance sont notés. Ces notes ont permis de recontextualiser chaque séance d'observation dans sa globalité. Ce journal de bord possède différents volets et selon chacune des étapes de la démarche de la recherche. Ainsi, un volet a servi à développer ce que certains auteurs appellent des "notes théoriques", c'est-à-dire tout énoncé, toute hypothèse, toute réflexion pour aider à dégager le sens de ce qui est observé et émettre des pistes de travail tant du côté théorique que méthodologique (Schatzman-Strauss, 1973: voir Huberman et Miles, 1991). Ont donc été notés dans ce carnet de bord, les perceptions, les suppositions, les sentiments, les questions et les pistes de problématique, de cadre conceptuel, de cadre méthodologique et de réflexions émergentes de lectures faites en cours d'expérimentation. 
Après chaque séance d'observation, chaque projet élaboré par un enfant, à deux, trois ou quatre a été photographié et une séance de discussion s'est déroulée avec chaque équipe afin de saisir les intentions/les réalisations, le choix des partenaires, le choix des thèmes, des scénarios et des rôles.

\subsubsection{La décision de l'échantillonnage}

Huberman et Miles (1991) indiquent que les questions de recherche préfigurent le cadre d'échantillonnage, c'est-à-dire l'endroit et les personnes qui permettent de trouver des réponses.

L'échantillonnage du point de vue de ces auteurs suppose une décision non seulement des sujets à observer ou à interroger mais aussi une décision sur le choix des milieux, des événements et des processus sociaux. La recherche qualitative nécessite un recentrage et une redéfinition des paramètres d'une étude, tout au long du travail de terrain, mais qui implique quand même une certaine sélection initiale. Le cadre conceptuel et les questions de recherche déterminent les centres d'intérêt et les frontières à l'intérieur desquelles sont choisis les échantillons.

Dans le cas de la présente recherche, le choix de l'échantillonnage porte sur quatre dimensions: le milieu: la classe maternelle; les acteurs: les enfants de 5-6 ans; les événements: l'activité de l'enfant au coin bloc, soit l'activité de construction et l'activité de jeu symbolique, les interactions sociales et, dans ce dernier cas, il s'agit des rapports interactifs favorisant davantage la réalisation d'un projet collectif de construction et de jeu symbolique social.

L'observation naïve s'est réalisée dans six classes maternelles de la région 02 . Trois rencontres ont été effectuées pour chacun des six groupes, dont deux rencontres où les enfants ont été filmés dans l'espace réservé au matériel bloc et une rencontre individuelle avec chaque enseignante, pour discuter du mode de fonctionnement de sa classe maternelle et de sa perception de cette aire de jeu et des activités des enfants dans cette aire. À la suite des données provenant de l'observation des enfants au coin bloc et des entrevues des enseignantes, certains critères sont apparus essentiels pour le choix d'une classe maternelle. 
Ces critères sont un coin bloc représentatif d'une bonne organisation matérielle: un espace assez grand pour la réalisation de divers projets, un contingentement de quatre enfants par séance, une bonne quantité de blocs en bois vernis pour la réalisation de projets à plusieurs, l'accès à des accessoires variés, la possibilité de choisir librement cet atelier et une période d'atelier d'une durée de 45 à 60 minutes. Deux critères non habituels dans le fonctionnement de cet atelier sont ajoutés: la présence simultanée de deux garçons et deux filles à chaque séance et une présence continue des mêmes enfants pour toute la durée de l'atelier. Une classe maternelle de 18 enfants a été retenue pour les fins de cette étude.

\subsubsection{L'instrument d'enregistrement des données}

Les enfants de la classe maternelle choisie ont été filmés pendant deux moments distincts de l'année scolaire. Le premier moment à l'automne et le deuxième au printemps. Ces deux moments ont été identifiés en collaboration avec l'enseignante comme étant des moments opportuns pour l'observation des enfants dans cet espace. À l'automne, l'observation a été étalée sur une période de deux mois et révèle l'activité des enfants en début d'année scolaire. Au printemps, les enfants ont également été observés sur une période de deux mois. Cette deuxième période d'observation permet de rendre compte d'une certaine maturité qui dévoile une activité de l'enfant au coin bloc assez différente et empreinte de nouvelles expériences. Vingt-quatre séances d'observation de l'activité des enfants au coin bloc ont été réalisées, d'une durée approximative de 45 à 60 minutes, pour chacune des séances.

Après chaque séance, un questionnement est proposé aux enfants portant sur leur séance de jeu. Par la suite, les productions des enfants sont photographiées.

La réalisation des 24 séances d'observation s'est effectuée avec une caméra manipulée par un cameraman professionnel, libérant ainsi l'observatrice de l'aspect technique de l'enregistrement des séances. De plus, un système d'enregistrement parallèle audio a été mis en place afin de minimiser le plus possible les contraintes techniques pouvant survenir et augmenter ainsi l'efficacité d'un repérage sur bande audio, advenant le cas d'une impossibilité sur bande vidéo. Par la suite, le cameraman a procédé à l'incrustation du temps en secondes sur la pellicule de chaque séance, afin de faciliter le repérage de l'inter- 
action des enfants lors de la reconstitution de la séance globale du verbatim des quatre enfants au coin bloc.

Parallèlement à l'observation par vidéoscopie, les relevés des observations et de différents événements pouvant intervenir pendant la cueillette des données sont notés. Cette prise de notes s'est avérée un guide utile lors du visionnement des cassettes vidéos, facilitant le repérage du contexte global de la séance et d'une première analyse succincte après chaque séance d'observation.

Le journal de bord a également été un instrument d'accompagnement non seulement à l'étape de la cueillette des données mais aussi aux étapes de l'analyse des données et de la rédaction de la thèse. Ce journal de bord a permis de consigner toutes les réflexions, les suppositions et les pistes de rédaction et d'analyses. Il a été un instrument très important qui a permis de saisir plusieurs cogitations en regard de la recherche et de soutenir des décisions de toutes sortes.

\subsubsection{La mise en situation pour chaque séance d'observation}

Les séances d'enregistrement se sont toutes déroulées à peu près de la même façon. La chercheuse et le cameraman arrivaient avant les enfants pour l'installation des appareils d'enregistrement (caméra, magnétophone et micros). À l'arrivée des enfants, l'observatrice participe à la causerie du matin avec l'enseignante et les enfants. Après la causerie, on procède à la période d'atelier. Dans cette classe, les enfants choisissent un atelier parmi les ateliers ouverts qui sont indiqués au tableau de programmation. Chaque enfant inscrit son choix d'atelier au tableau de programmation, en plaçant l'étiquette de son nom à l'endroit disponible dans chaque atelier.

Pour l'atelier des blocs, la consigne, comme déjà mentionné, a été modifiée. L'enfant qui choisit cet atelier doit y demeurer toute la période en respectant de plus la présence de quatre enfants soit deux garçons et deux filles. Dans le choix de l'atelier des blocs, la formation naturelle des groupes a été respectée, tout en s'accordant le privilège d'apporter des modifications, si cela s'avère nécessaire. Étant donné le nombre de garçons (13) et de filles (6), on demandait aux enfants de respecter la consigne 2-2. Mais 
comme on privilégiait aussi la formation naturelle des associations, cette consigne n'a pas toujours été respectée.

\subsection{La planification de l'analyse qualitative des données}

Dans le processus de la recherche qualitative, l'analyse des données est un processus qui doit normalement se réaliser simultanément au processus de cueillette des données. Dans le processus de recherche d'un contexte doctoral, il est admis que l'auteure assume, le plus souvent seule, toutes les étapes de la réalisation méthodologique de la recherche. Ainsi, la phase de cueillette et de l'analyse simultanée des données qualitatives s'en trouve quelque peu modifiée et ce, par diverses contraintes d'ordre technique et institutionnel.

Les premières contraintes sont d'abord institutionnelles puisque dans le cas qui nous importe, la cueillette des données s'effectue sur une longue période, une année scolaire. Après avoir planifié deux moments opportuns dans cette année scolaire et des dates possibles d'observation permettant l'analyse entre chacune des séances, la première modification a été celle du calendrier planifié, tenant compte des congés, des activités spécialisées et des sorties. Les séances d'observation se retrouvent donc dans un temps très rapproché et ne peuvent faire l'objet d'une analyse exhaustive.

La deuxième contrainte est liée au temps réel de visionnement des cassettes, à la transcription du verbatim et des actions sur le matériel de la part de chacun des quatre enfants pour chaque séance et par la suite, de l'analyse de ces informations. Il était donc difficile d'assurer une analyse complète entre chacune des séances d'observation. Cependant, en effectuant un visionnement de la séance d'observation pour recadrer le contexte global, en notant différents ajustements techniques pour la prochaine séance, en consignant un certain nombre de questionnements et de thématiques, il a donc été possible d'assurer un minimum de continuité entre les séances d'observation.

\subsubsection{Les stratégies globales de mise en forme des données}

Avant l'analyse systématique des données, il faut s'assurer d'un cadre de présentation permettant leur recouvrement et leur condensation. Chaque séance a fait l'objet d'une 
transcription du "verbatim" de chaque enfant (quatre enfants par séance d'observation) et d'une transcription des actions de l'enfant sur le matériel bloc et sur le matériel accessoire. Cette transcription a été guidée par le temps consigné sur chaque bande magnétoscopique. Par la suite, la transcription de chaque enfant a été fusionnée selon les partenaires de jeu afin de reconstituer la séance de jeu dans le contexte global de la situation.

La trame d'analyse des données utilisée dans cette recherche, de type qualitative, est issue de la méthode d'analyse de contenu de L'Écuyer (1990) et combinée à la méthode d'analyse de Huberman et Miles (1991).

Le modèle retenu est donc un modèle mixte (L'Écuyer, p.199) qui tient compte à la fois des catégories issues du cadre théorique et des catégories émergentes de la situation observée. En effet, un grand nombre de concepts théoriques a été retenu pour mettre en forme une certaine trame évolutive de l'activité des enfants avec le matériel. Ces concepts théoriques se traduisent en catégories et permettent de déterminer les éléments à tenir compte lors des diverses lectures des corpus d'enregistrement des bandes magnétoscopiques. Par la suite, une liste de concepts émergents a été dégagée de ces mêmes lectures afin de șaisir la dynamique globale de l'activité de construction et de l'activité de jeu symbolique et des différentes interactions sociales mises à contribution dans l'activité des enfants avec le matériel bloc.

Ces deux types de catégories, théoriques et émergentes, se devraient de regrouper les données relatives aux stades d'évolution, aux composantes structurales et aux interactions sociales. L'analyse de ces stratégies interactives essentielles et nécessaires à la mise en forme de l'activité des enfants avec le matériel bloc, nous permettra de saisir la dynamique sociale et cognitive particulière à cette activité.

Cette méthodologie s'est largement inspirée des travaux de l'équipe du Centre de Recherche de l'Éducation Spécialisée et de l'Adaptation Scolaire (CRESAS) en France. Le regard qualitatif que ces chercheurs posent sur leurs données d'observation, des interactions sociales de très jeunes enfants dans leur jeu de fiction, est un outil original pour notre étude (Stamback, Sinclair, 1990). Ces travaux inspirent également le développement de voies nouvelles et innovatrices dans le domaine de la recherche en éducation, en 
se centrant sur la description et la compréhension de la qualité des environnements et des interventions pédagogiques.

Chaque question générale de recherche a suscité un questionnement et des modalités de cueillette et d'analyse. En effet, pour répondre à la première question générale: quelle est la nature de l'activité de l'enfant que le matériel bloc suscite chez l'enfant?, il fallait se demander quelles sont les particularités de l'activité de construction? quels sont les produits de cette activité? est-ce que le jeu symbolique est présent dans l'activité de construction?, et si oui, quelles sont ses caractéristiques et quels sont ses modes d'organisation?

La deuxième question générale de recherche: est-ce que ce matériel bloc et les activités qu'il suscite contribuent au développement cognitif et social de l'enfant qui fréquente la classe maternelle?, nous a amenée à un questionnement plus spécifique afin de prendre en compte les étapes d'évolution de l'activité de construction, les composantes structurales spécifiques à l'activité de jeu symbolique reliées à la nature du médium bloc et les interactions sociales que les activités de construction et de jeu symbolique provoquent.

Ces deux types d'activités ont été soumis à l'analyse. D'abord sur le plan physique, l'activité de construction a exigé d'examiner les différentes stratégies interactives qui rendent possible cette activité, les différents procédés que les enfants mettent en oeuvre pour assurer cette construction partagée et les éléments indispensables qui la soustendent. Ainsi, quels sont les éléments privilégiés dans cette dynamique sociale qui font que deux, trois ou quatre enfants déploient tant d'efforts pour réaliser une construction collective?

Pour ce faire, dans chacune des séances d'observation, les différents épisodes de jeu où l'accent "construction" était au coeur de la dynamique ont été relevés. Par la suite, l'intérêt s'est porté sur les éléments communs et particuliers qui sous-tendent l'organisation et le déroulement de cette activité collective.

De plus, comme nous étions également intéressée par l'aspect symbolique que génère cette activité, nous avons relevé les épisodes de jeu symbolique, autant ceux intégrés à l'intérieur des épisodes de l'activité de construction que ceux subordonnés ou indépendants de ces épisodes. Cette analyse nous a permis de dégager la particularité ou l'uni- 
formité de l'activité de jeu symbolique générée par l'activité de construction avec le matériel bloc.

Les spécificités de ces questionnements ont donc exigé des modalités particulières d'analyse qui seront présentées dans le quatrième chapitre, consacré à la mise en forme des données. 


\title{
CHAPITRE IV
}

\section{D'UNE MISE EN FORME DES DONNÉES \\ AUX PREMIÈRES PISTES DE COMPRÉHENSION DE L'ACTIVITÉ DE L'ENFANT SUSCITÉE PAR LE MATÉRIEL BLOC}

\begin{abstract}
Ce chapitre présente la mise en forme des données de recherche relatives aux trois questions découlant de la problématique de l'activité que le matériel bloc suscite chez l'enfant.
\end{abstract}

Pour chacune des trois questions de recherche, sont identifiés les éléments indicateurs pris en compte pour une mise en forme des données. Par la suite, ces éléments indicateurs sont regroupés sous des thèmes de réflexion afin de mettre en évidence la contribution potentielle de l'ensemble des éléments identifiés et émergents. Chaque thème de réflexion relate, dans un premier temps, les aspects théoriques qui sous-tendent ces indicateurs et, dans un deuxième temps, regroupe les observations relatives à ces éléments. Cette analyse trace les bases de compréhension et d'interprétation des données liées à chacune des trois questions. Cette lecture interprétative sera présentée au cinquième chapitre.

\subsection{Première question de recherche: stades d'évolution de l'activité de construction}

Dans son activité de construction, l'enfant, à partir des blocs, invente des objets et des événements. Faisant référence aux écrits de l'approche développementale dans la perspective psychologique, Johnson (1974) identifie certains procédés de construction correspondant à des stades ou étapes d'évolution de l'activité de construction, d'où la 
formulation de la première question de recherche qui, rapellons-le, se formule comme suit:

À quel stade ou à quelle étape d'évolution de l'activité de construction se situent les enfants de 5-6 ans qui fréquentent la classe maternelle?

\subsubsection{Les modalités spécifiques d'analyse des données de la pre- mière question de recherche}

Pour répondre à cette première question de recherche, chacune des neuf séances retenues a été visionnée. Les informations recueillies sont complétées à l'aide des notes de terrain prises en séance d'observation participante et des notes élaborées lors d'un premier visionnement global à la phase de la cueillette des données et d'un second visionnement, lors de la phase de la transcription du verbatim.

Chaque séance d'une durée approximative de 45 à 60 minutes est visionnée à quatre reprises pour observer séparément chacun des quatre enfants dans son mode d'organisation de l'activité de construction. Par la suite, un résumé de la séance est réalisé selon un certain nombre d'éléments constitutifs permettant de mieux cerner le stade d'évolution de l'activité de construction. Ce visionnement permet de mettre en évidence les actions de l'enfant avec ce matériel lorsque ce dernier s'occupe à une activité de construction.

À partir de ces données, un tableau des différents éléments indicateurs de l'activité de construction est élaboré afin d'en saisir les aspects significatifs pouvant répondre à la première question de recherche portant sur les stades d'évolution de l'activité de construction.

Pour chacune des neuf séances retenues et pour chaque enfant, nous avons observé un ensemble d'éléments indicateurs, lesquels sont présentés au tableau 4.1.

Afin de bien saisir l'essence même de ces éléments indicateurs eu égard au stade d'évolution des enfants de 5-6 ans, les données de chacun de ces éléments sont examinées à partir de quatre thèmes: les stades d'évolution de l'activité de construction, les produits de l'activité, le temps consacré à l'activité de construction dans une séance d'atelier au coin bloc et les formes de participation sociale. 


\section{Tableau 4.1}

Ensemble des éléments indicateurs retenus

relatifs aux stades de l'évolution de l'activité de construction.

1. Stade d'évolution et complexité des productions.

\section{Temps total de la séance/ temps consacré à l'activité de construction.}

7. Utilisation d'accessoires.

10. Type de participation sociale au projet de construction solitaire, dyadique, triadique, ou à quatre.
2. Productions annoncées et changements en cours d'activité.

5. Moments distincts de la répartition du temps pour l'activité de construction.

8. Enfant-acteur ou utilisation de personnages $\mathrm{mi}$ niatures.
3. Productions finales/productions annoncées/productions réalisées.

6. Nombre de blocs utilisés.

9. Représentations symboliques:

- transformations matérielles

- transformations symboliques.

\section{Thème 1- Les données relatives aux stades d'évolution de l'activité de construction}

Avant d'aborder les données associées aux stades d'évolution de l'activité de construction des enfants de 5-6 ans observés dans cette étude, rappelons la trame de fond théorique qui a servi de base à l'analyse des différentes productions des enfants.

Selon les données identifiées par Johnson (1974) et raffinées par Apelman (1984), Dodge (1979a) et Santa (1980), les enfants qui fréquentent la classe maternelle se situeraient aux stades 5 et 6 .

Les caractéristiques du stade 5, apparition de modèles décoratifs, se traduisent par une certaine aisance à utiliser les techniques du pont et de l'enclosure; l'apparition de modèles variés et de productions inusitées sans identification; l'observation de la symétrie et de l'équilibre; la répétition du modèle de base et une rareté des commentaires sur la construction.

Au stade 6, stade de la représentation et de l'identification, les enfants ont tendance à nommer les productions réalisées et à répéter les procédés acquis (la tour, la rangée, le pont et l'enclosure), ce qui ne signifie pas pour autant qu'il y ait nécessairement ressemblance entre les productions réalisées et le nom attribué à cette production. Peu à peu, 
nommer les productions devient une chose usuelle et leur nom est surtout relié à la fonction de cette production et les enfants les annoncent avant la réalisation. Par la suite, l'utilisation des produits de l'activité de construction sert d'amorce à l'activité de jeu symbolique et ce, lorsque l'enfant a suffisamment bien maîtrisé les différentes techniques de construction. À partir de ce moment, il semblerait que le matériel bloc devient secondaire dans l'activité de l'enfant.

À ce stade 6, appelé le jeu dramatique par Santa (1980) et la représentation par Alpeman (1984), les productions des enfants reproduisent ou symbolisent des structures connues de ces derniers. À ce stade, on observe une forte impulsion vers le jeu dramatique autour et à partir des productions. Réalisées avec le matériel bloc, celles-ci serviraient de cadre de référence pour le jeu dramatique qui deviendrait plus coopératif et interactif. Souvent, les enfants vont introduire d'autres types de matériel servant de complément au jeu en cours d'élaboration. Les blocs demeurent cependant le matériel central de l'activité. Finalement, la répétition des procédés est mise en évidence et la complexité des productions se traduit dans les détails apportés aux productions.

De ces données théoriques, nous proposons trois pistes d'observation et d'analyse du stade de développement de l'activité de construction. La première piste découle de l'observation des différentes productions réalisées en cours de séance et du projet terminé. La deuxième piste consiste à analyser les composantes privilégiées dans la réalisation des différentes productions. Enfin, la troisième piste regarde la communication verbale de l'enfant accompagnant ses nombreuses actions sur le matériel qui, évidemment, témoigne et traduit les intentions de l'activité de construction.

\section{- Observation des différentes productions}

À l'examen de l'ensemble des productions des enfants, on remarque que chacune est élaborée avec un nombre considérable de blocs (60 et plus) de toutes formes, grandeurs et longueurs. Nous pouvons considérer que ces productions sont le fruit d'une maîtrise des différents procédés acquis antérieurement dans leur activité de construction. En effet, les enfants utilisent abondamment les procédés de tour, de rangée, de ponts et d'enclosures, comme paliers de base d'une multitude d'arrangements des plus variés les uns que les autres et qui font appel à la répétition et à la complexification de ces procédés de base pour l'élaboration de productions de plus en plus inusitées et sophistiquées. La 
lecture des illustrations suivantes témoigne de la maîtrise des différents procédés utilisés dans l'activité de construction par les enfants de l'étude.

A) Procédé de base: la tour, la rangée

L'utilisation et la répétition des procédés de la tour et de la rangée dans la complexité d'une production (photos 1 à 6).

Photo 1

Séance 13

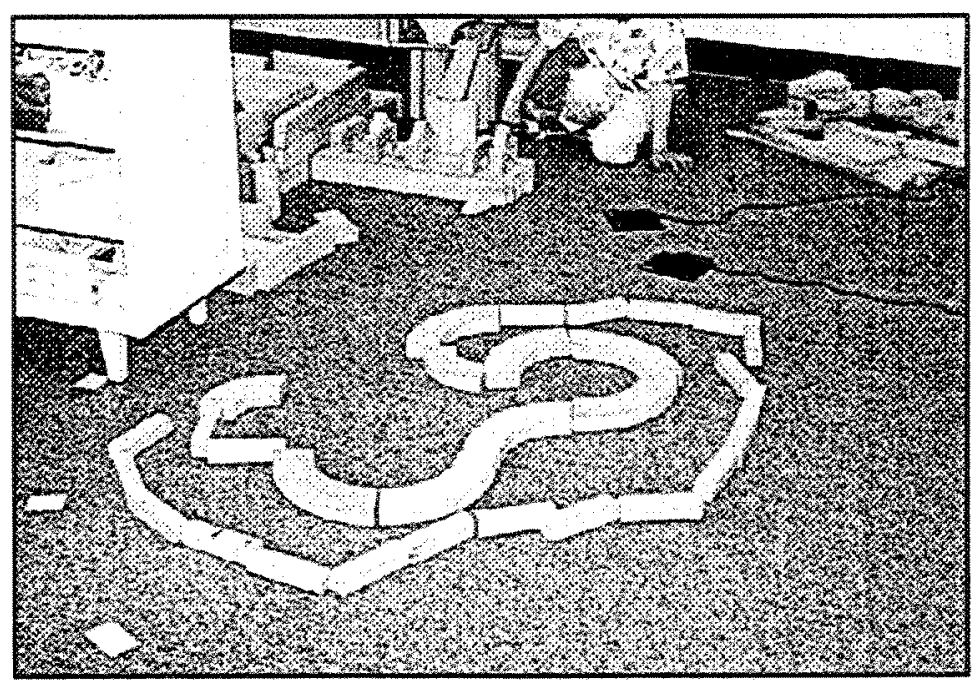

Photo 2

Séance 8

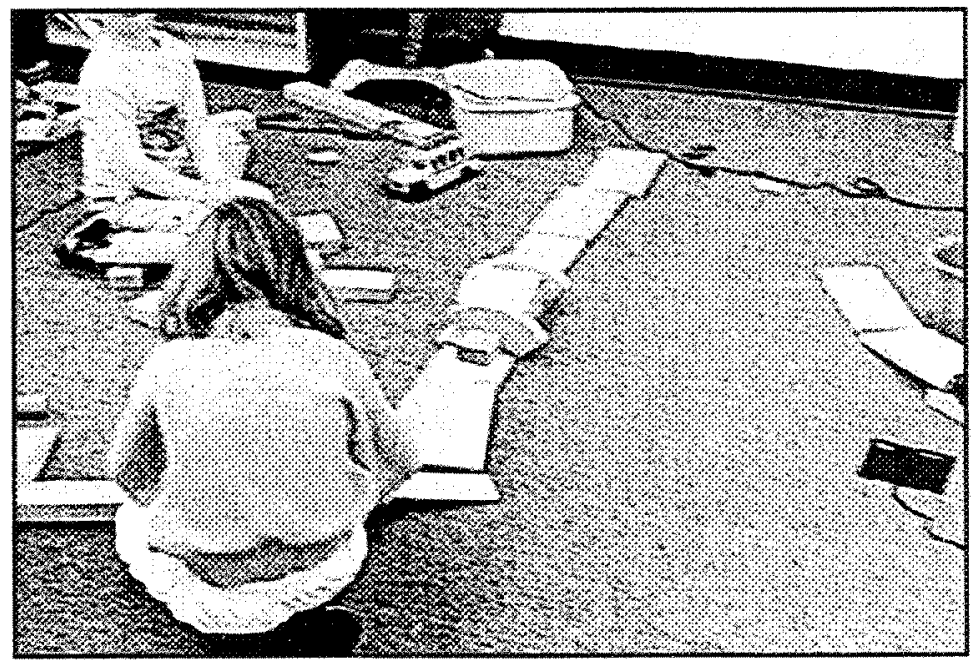


Photo 3

Séance 8

Photo 4

Séance 9

Photo 5

Séance 8
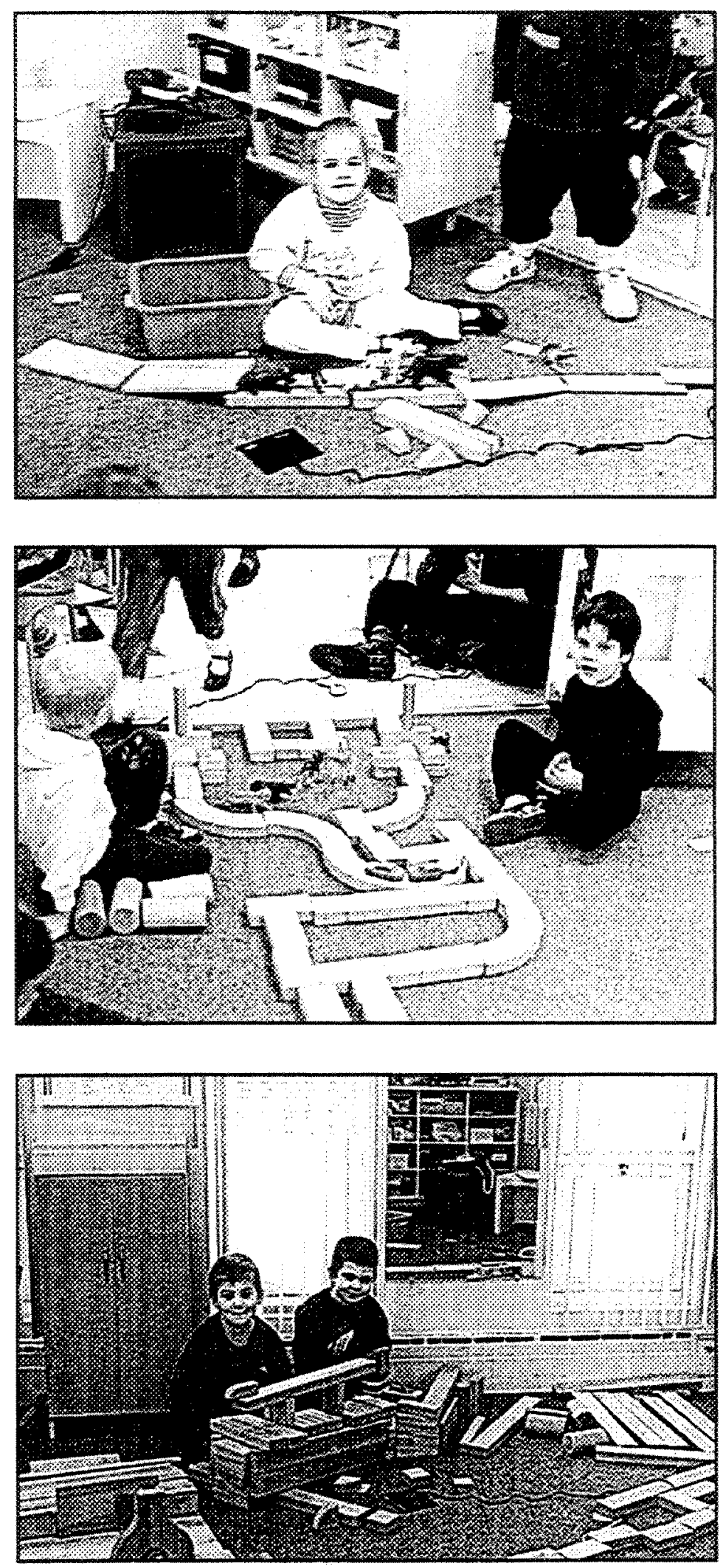
Photo 6

Séance 13

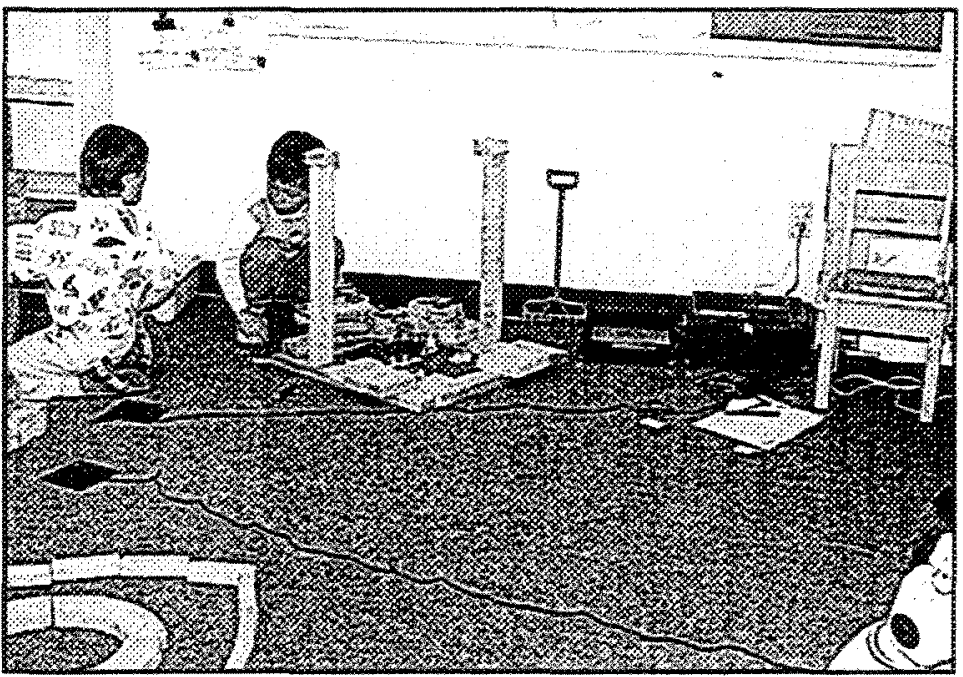

B) Procédé de base: le pont

L'utilisation et la répétition du procédé de base du pont, pour la réalisation d'un édifice à plusieurs étages.

Photo 7

Séance 2

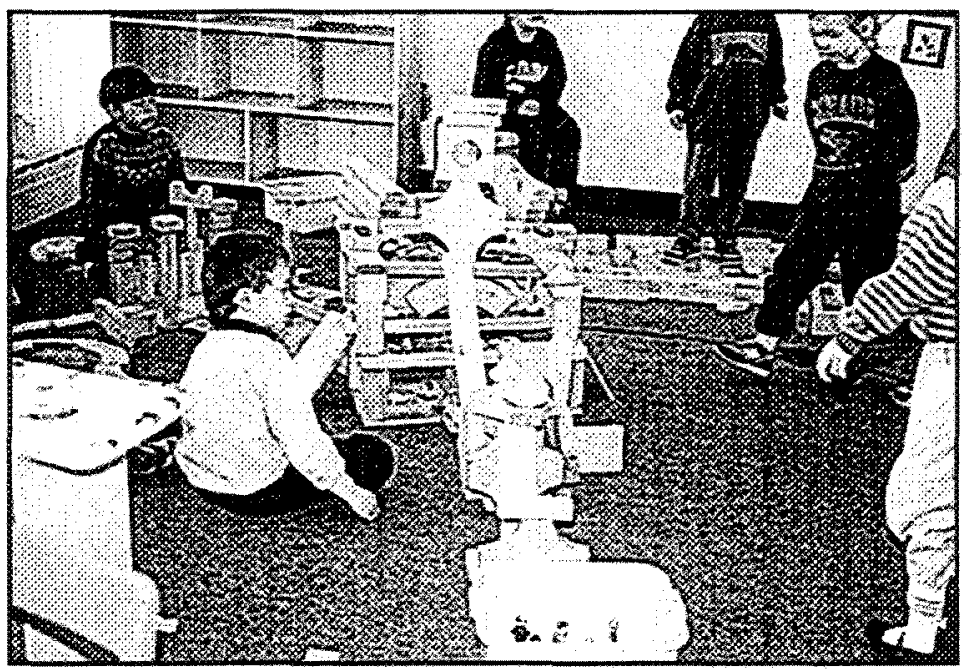


C) Procédé de base: l'enclosure

L'utilisation du procédé de l'enclosure de plus en plus élaborée pour réaliser divers espaces d'une même production.

Photo 8

Séance 10

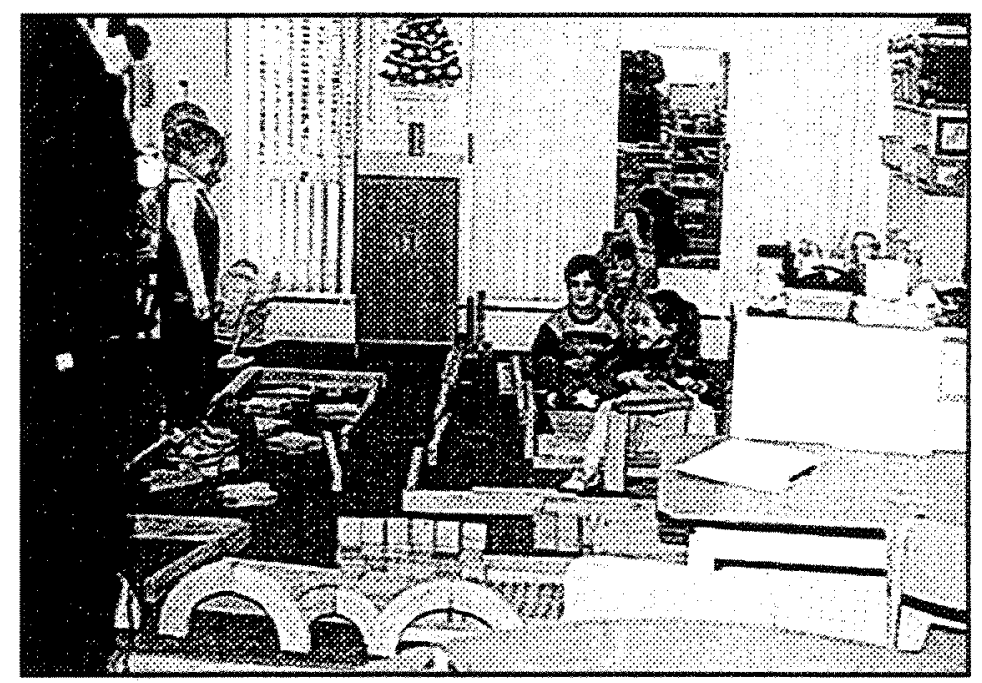

Photo 9

Séance 6

Photo 10

Séance 6
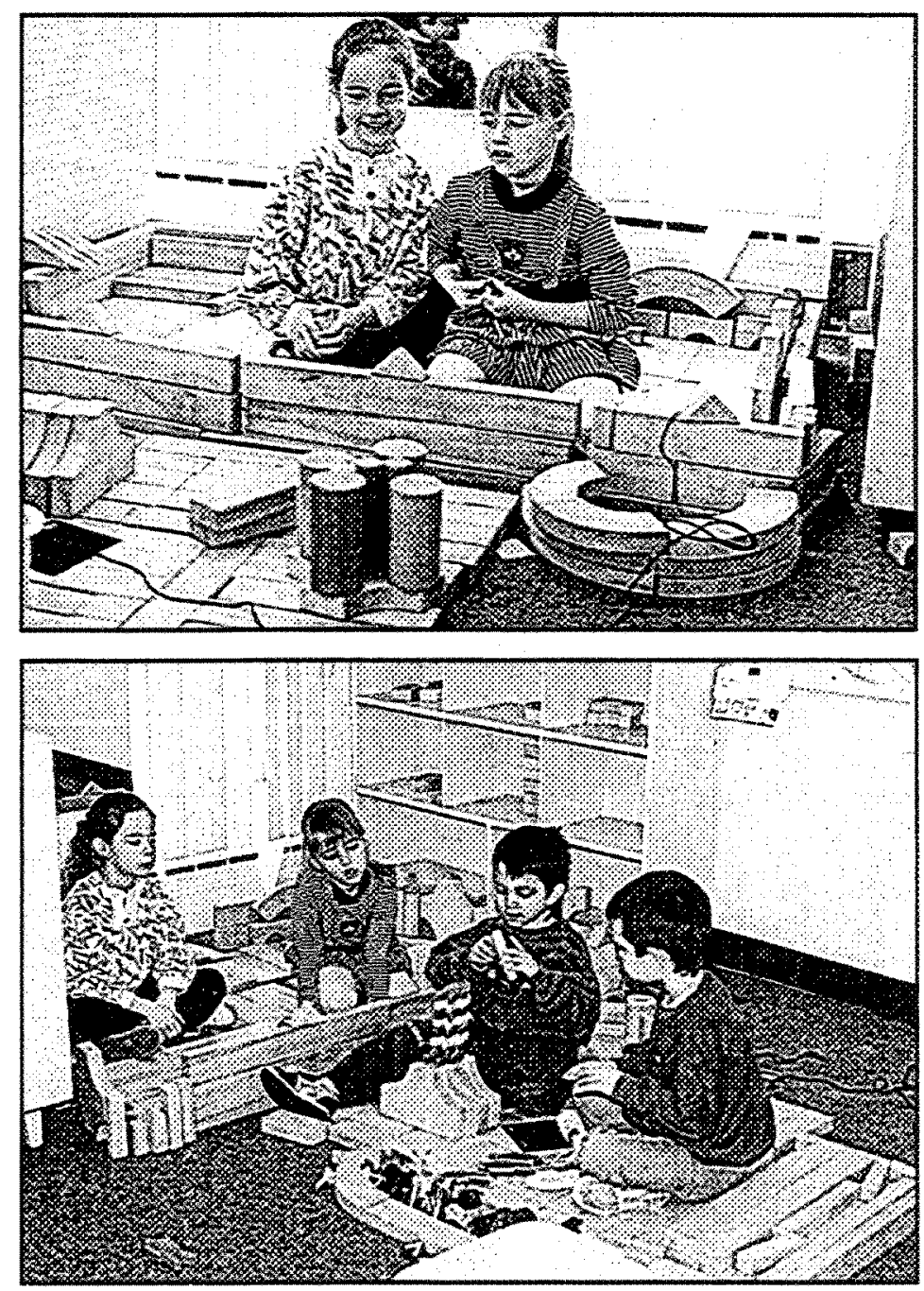
Photo 11

Séance 6

Photo 12

Séance 6

Photo 13

Séance 10
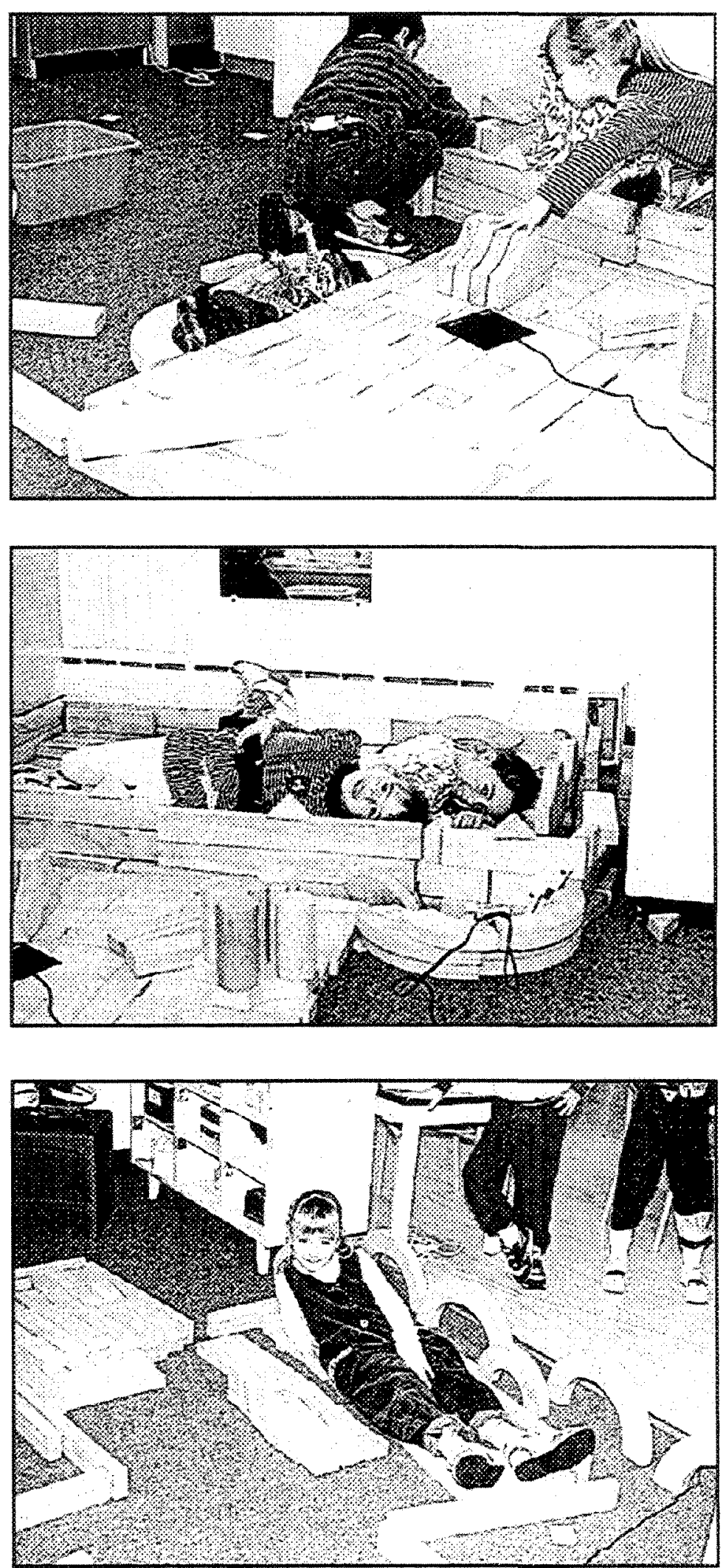
Photo 14

Séance 11

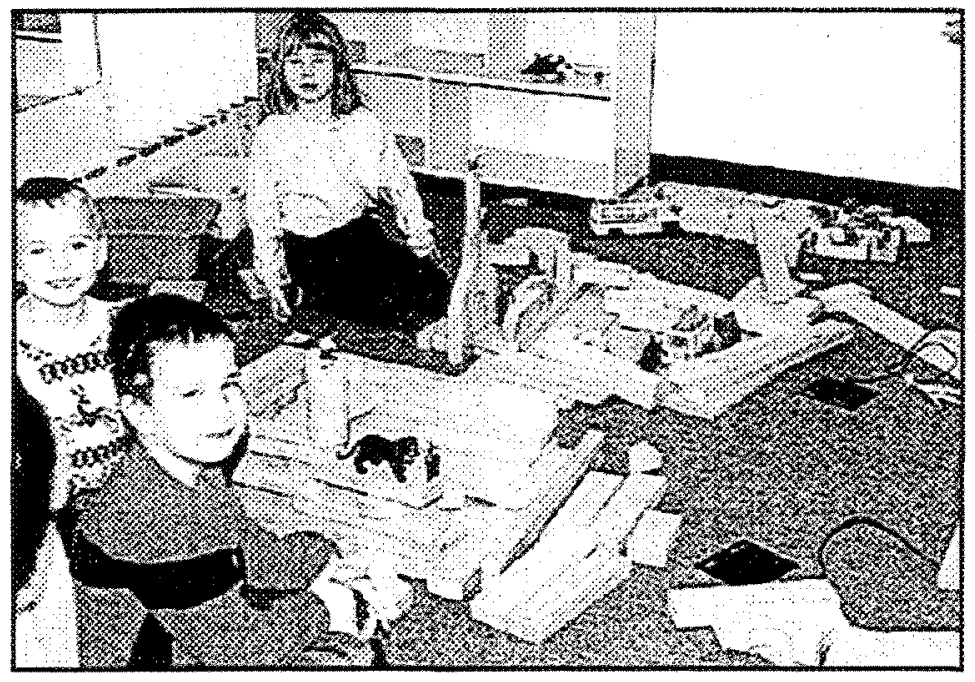

De plus, notons que les blocs tels que triangle, carrefour, cylindre, chaussée, quart de cercle, $Y$ connecteur, courbe elliptique, sont utilisés dans plusieurs productions comme éléments décoratifs pour compléter et enjoliver les productions (photos 15 à 17).

Photo 15

Séance 11

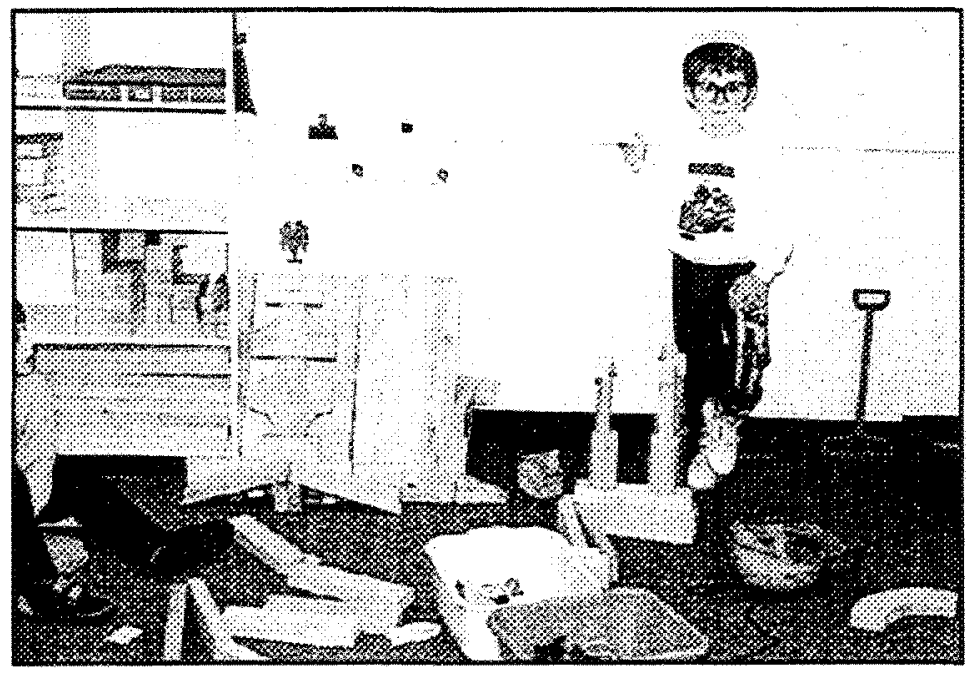


Photo 16

Séance 13

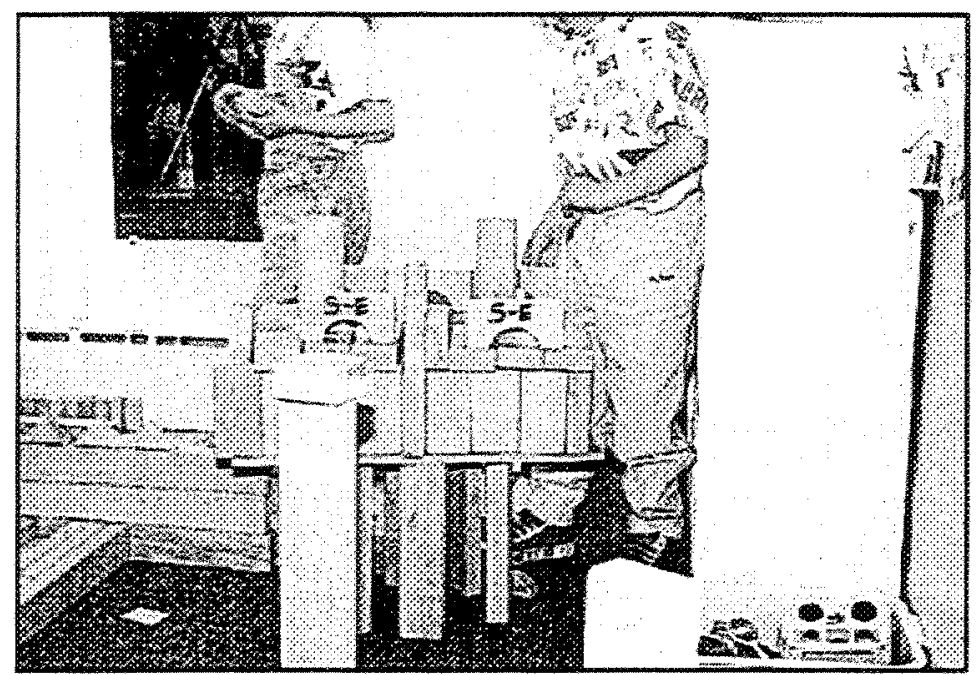

Photo 17

Séance 5

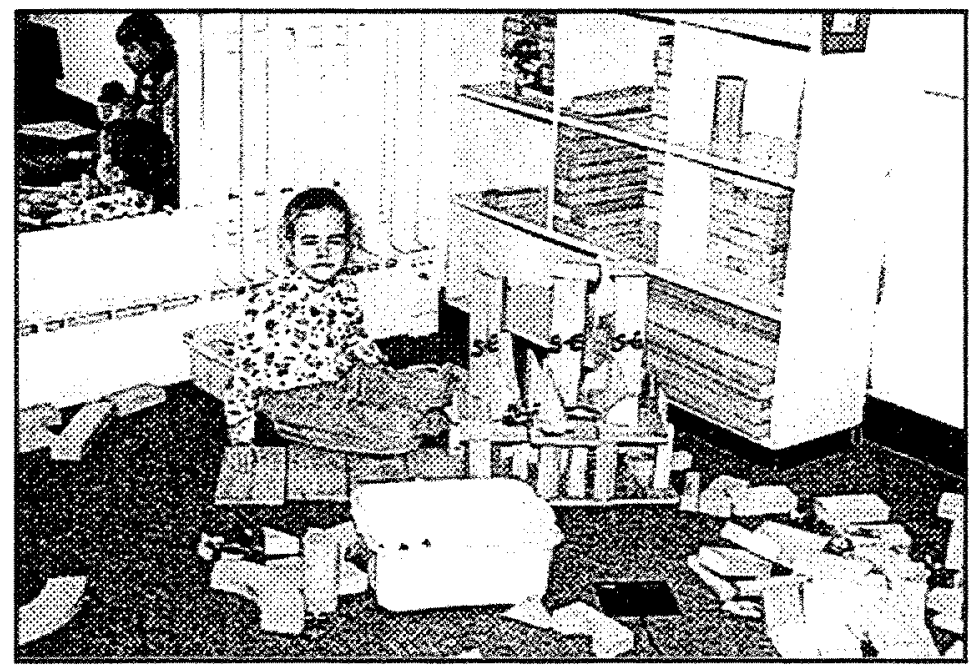

Et finalement, l'utilisation simultanée et combinée de divers procédés de construction tels que la tour, la rangée, le pont et l'enclosure dans l'élaboration de productions plus complexes (photos 18 et 19). 
Photo 18

Séance 2

$\mathrm{n}^{\circ} 1$ : fait référence au procédé de rangée

n०2: fait référence au procédé de la tour

n³: fait référence au procédé du pont

$n^{\circ}$ 4: fait référence au procédé de l'enclosure

$n^{\circ}$ 5: fait référence aux modèles décoratifs.

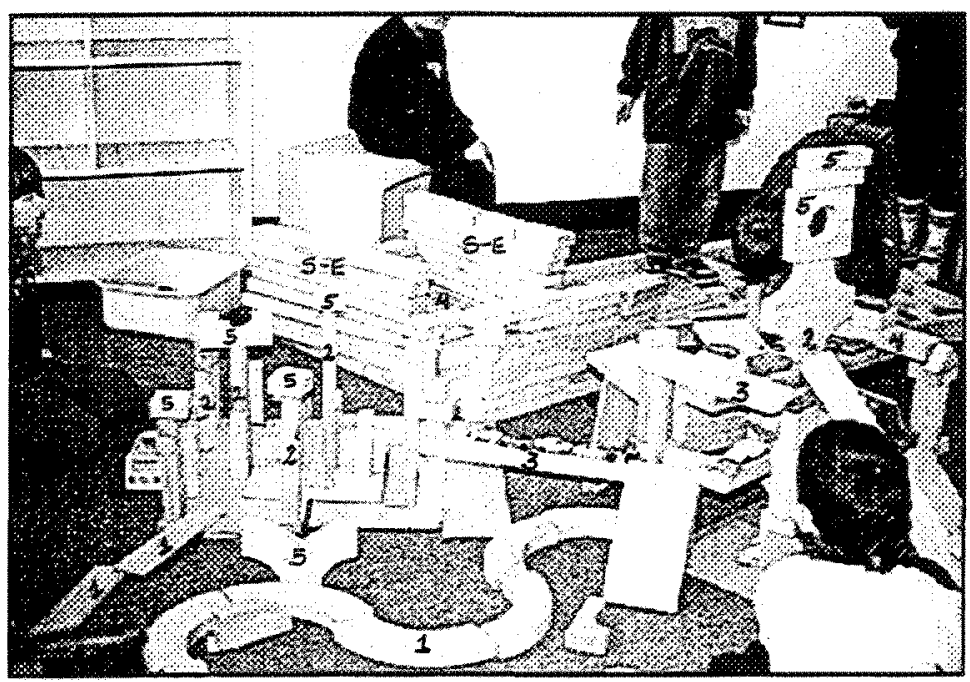

Lettres S-E:

font référence aux processus de symétrie et d'équilibre.

Photo 19

Séance 13

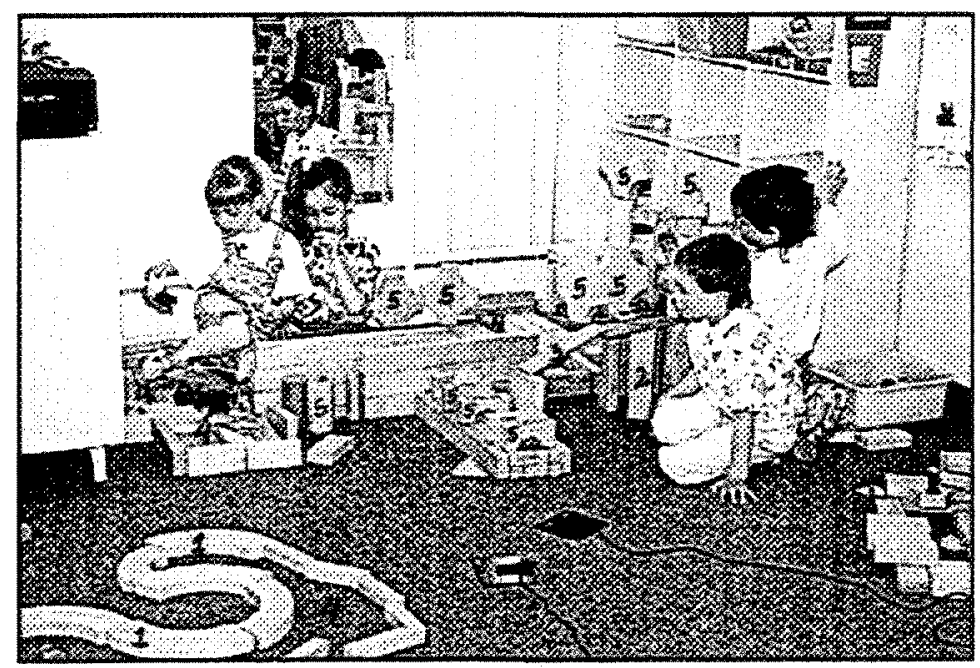

- Observation des composantes privilégiées: symétrie et équilibre

La deuxième piste d'analyse concerne l'examen des productions el l'observation des composantes utilisées par l'enfant pendant l'activité de construction et révèle deux éléments importants dans cette activité: la symétrie et l'équilibre. En effet, lorsque l'enfant s'affaire à réaliser sa production, il est constamment soucieux d'ériger les parties de sa production en respectant la symétrie et l'équilibre. Ce souci se traduit par la réalisa- 
des divers côtés de sa production (côté gauche, côté droit, devant, derrière) en construisant alternativement chacune des facettes avec la préoccupation que celles-ci soient reproduites selon le même modèle initié. Cet élément de symétrie se traduit par l'utilisation des mêmes types de blocs pour chacun des côtés, pour la hauteur, pour la largeur et les mêmes éléments de décoration assurant ainsi la symétrie et l'équilibre de la production. Les photos 2, 6, 16, 17 et 18 illustrent le souci de symétrie et d'équilibre dans les diverses productions des enfants (voir l'identification de ces processus par les lettres S-E). L'exemple suivant traduit les processus de symétrie et d'équilibre dans les actions des enfants lors de l'activité de construction.

SEA3-ENF4

04:19:28 Il prend trois modules qu'il place sur le mur de droite de sa construction. Il prend un autre module et continue à ériger son mur. Il prend un autre module.

05:04:00 Il prend un autre quadrangle qu'il place sur le mur, côté gauche de sa construction, pour compléter sa cinquième rangée.

05:12:28 Il prend un autre quadrangle qu'il place sur le mur, côté droit de sa construction, pour compléter sa cinquième rangée.

05:19:03 Il prend un autre quadrangle qu'il place sur le mur arrière de sa construction pour compléter sa cinquième rangée.

05:24:20 Il place tous les blocs de ses quatre murs bien droits.

05:30:01 Il prend un quadrangle qu'il place sur le mur arrière de sa construction pour compléter sa cinquième rangée. (Afin de s'assurer que tous les murs sont de la même hauteur).

05:44:00 [Continue ...].

07:33:21 Il se lève et fait un tour complet en vérifiant si tous les blocs sont bien droits et les murs de la même hauteur.

De plus, l'enfant traduit aussi verbalement son action de symétrie et d'équilibre par différents énoncés, tels que:

SEA2-ENF3

08:02:19 I, 2, 3, 4 (d'un côté); 1, 2, 3, 4 (de l'autre côtê), comme ça, comme ça, ton mur faudrait t'a r'mettre, faudrait, à côté un bloc, faudrait l'a remettre un autre bloc, faudrait t'en remettre un autre (en montrant le mur, il regarde s'il y a le même nombre de blocs qui forment la partie avant de sa construction).

08:02:19 Ah! on a pu, oh ben tend une minute, on va mettre ceux-là d'même, donne-moi dont ceux là, ça va être [...]. (Il place quatre petits blocs sur les planches afin d'obtenir une planche de plus pour que les deux murs soient de la même hauteur).

SEA2-ENF4

12:49:11 1,2,3 (compte les blocs de son mur à l'horizontal en pointant et touchant chaque bloc). Je vais aller chercher d'autres blocs, on a besoin. (Il revient avec des blocs pour continuer à ériger son mur). (Il place encore deux blocs en pente et en symétrie de chaque côté).

14:08:07 Va resserrer lui, va resserrer lui (il donne le bloc à son partenaire parce que le mur est maintenant à la même égalité que les deux autres). 


\section{- Observation de la communication verbale}

La dernière piste d'observation et d'analyse de l'évolution du stade de développement de la construction concerne la communication verbale de l'enfant, laquelle est un des éléments significatifs de cette activité.

Dans l'évolution des stades de développement de cette activité, la communication verbale a été prise en compte au stade 6 dans l'élaboration de l'activité de jeu symbolique. Cependant, aucun auteur n'avait considéré cette communication verbale dans le processus même de l'activité de construction proprement dite.

Dans cet ordre d'idées, lors de la phase de la transcription du "verbatim" pour chacun des enfants et à l'étape d'analyse, la communication verbale est apparue comme un élément marquant et dominant de l'activité de construction. Cette communication est présente et très accentuée lorsqu'il y a deux enfants et plus qui s'affairent à la même production. Elle l'est également dans le cas des projets individuels; cependant, la fonction des énoncés est de nature différente.

En effet, lorsque les enfants partagent le même espace de jeu au coin bloc, mais que leur activité de construction est une activité plutôt parallèle, l'enfant s'amuse seul, mais l'activité qu'il choisit le place en compagnie d'autres personnes; les enfants jouent à des jeux similaires avec le même matériel et les mêmes accessoires, mais sans véritables interactions. Les données recueillies révèlent que les enfants jouent les uns à côté des autres plutôt que les uns avec les autres.

Dans cette forme de participation sociale, les énoncés observés peuvent se regrouper de la façon suivante:

- Énoncés pour faire part de leur intention de projet aux autres ou à lui-même: SEA6-ENF2:

15:17:08 Tiens, ça va être une grosse base.

SEA6-ENF4:

00:15:00 Oh ben, moi, je fais un beau château. 
- Énoncés pour donner une appréciation sur sa propre production:

SEAl-ENF1

04:28:00 Regarde, j'ai fait une belle route en maudit!

SEA6-ENF1

16:23:24 Je l'aurai jamais.

SEA6-ENF4

29:36:08 Y est beau hein! mon royaume! Y'est en train de se faire bâtir.

$\mathrm{Ah}$ ! je pense qui va être trop gros.

- Énoncés pour donner une appréciation sur la production d'un pair:

SEA1-ENF2

03:34:07 (Il se lève et regarde la construction de l'autre). Hé, ça va tomber, il faut que tu tournes toute, parce que ...

(Il regarde la construction de l'autre qui est en train de tomber). C'est fragile, hein!

\section{SEA6-ENF4}

02:42:11 Aïe! est belle ton autoroute!

- Énoncés pour demander du matériel

- Énoncés pour indiquer un ajustement:

SEA1-ENF3

01:16:24 Aie! (nom de l'enfant), tu pourrais tasser encore, t'es direct sur nous autres.

- pour demander de l'aide:

\section{SEA1-ENF3}

Aïe! (prénom de l'enfant, deux fois) viens donc m'aider à placer mes autos comme il faut.

\section{SEA6-ENF4}

00:02:28 Aïe! Aïe! (prénom de l'enfant, deux fois), veux-tu m'aider à faire mon château?

- Énoncés pour une mise en garde:

SEA1-ENF1

03:55:00 Faites attention parce que c'est fragile.

04:06:00 Faites attention à ces affaires-là, là, je l'ai pas fait pour rien.

SEA1-ENF3

04:19:26 Aïe! vous voyez pas les autos vous (parce que les autres enfants du coin bloc vont au casier de rangement chercher des blocs en passant par-dessus les voitures de l'enfant 3 ).

- Énoncés pour le respect des règles:

SEA6-ENF4

45:49:16 Aïe! C't'à, les blocs sont à tout le monde. 
- Énoncés pour proposer une idée à un pair:

SEA1-ENF1

04:51:16 (Parle à l'autre en lui montrant quelque chose). Regarde, enlève cette planche-là: pour faire un toit.

05:04:00 Met une porte là, o.k., et tu vas mettre une petite porte là.

05:08:11 Comme ça, la petite porte, tu vas pouvoir rentrer.

SEA1-ENF3

09:04:26 Ben fais donc une maison comme tantôt.

- Énoncés pour entrer en interaction par le biais du matériel:

SEA1-ENF3

07:35:24 Aïe! comment on fait pour aller au garage.

07:49:13 Aie! regarde ouste que t'ai. Peut rouler.

08:44:27 (Prénom de l'enfant, deux fois). Tu prends toute le paquet là. Tu vas toutes les mettre dans ton garage.

10:08:17 (Prénom de l'enfant, deux fois). Y va partir de reculons (en montrant sa voiture).

- Énoncés pour entrer en interaction par le biais de la production:

SEA1-ENF4

12:37:21 (Donne une tape à l'autre sur les jambes). (Prénom de l'enfant). Regarde ma maison.

Également, on dénote quelques cas d'énoncés reliés aux actions qui traduisent, au fur et à mesure, les processus d'élaboration de la production et du scénario:

SEA6-ENF1

23:34:23 Fermer, tous les voleurs sont là-dedans.

SEA6-ENF4

14:30:26 Haye, hein! Ben moé ch'ten train de bâtir. Chu t'en train de bâtir.

43:02:25 Ah non, non! C'tes autos-là y vont en arrière et pis c'tes autos-là, y vont les mettre juste à côté.

Par contre, lorsque les enfants s'affairent au même projet de construction, deux grandes catégories d'énoncés se dégagent dépendant si les enfants s'engagent dans une forme de participation sociale de type associatif ou de type coopératif.

Dans l'activité de construction de type associatif, les enfants construisent avec d'autres enfants en échangeant du matériel et des accessoires. Ils se suivent et s'imitent; cependant, il n'y a pas de véritables divisions des rôles ou d'organisation dans les relations sociales concernées. Chaque joueur élabore à partir de la même production en agissant un peu comme il veut, sans subordonner ses intérêts ou ses actions à ceux du groupe ou d'un ensemble. 
Dans cette activité associative de construction, les énoncés qui se dégagent sont formulés, soit:

- pour annoncer une intention de projet, mais sans avoir nécessairement entente des partenaires pour l'élaboration du projet, donc chacun réalise son intention, mais sur la même base de construction;

- pour inviter l'autre comme partenaire dans son activité de construction;

- pour demander conseil à l'autre;

- pour décrire chacune des actions dans l'élaboration de la production, donc verbalisation de la démarche.

Les exemples représentant ces énoncés seront présentés à l'analyse des données de la troisième question de recherche.

Dans l'activité de construction de type coopératif, nous avons vu que les enfants s'amusent dans un groupe organisé ayant un but collectif prédéterminé. La réussite d'une telle forme d'activité de participation nécessite une division de l'action et une distribution de rôles précis aux membres du groupe, l'organisation de l'action suppose une entente réciproque et une complémentarité des efforts de chacun des partenaires.

Dans cette activité de construction coopérative, les énoncés étaient tous orientés vers un but d'entente et de partage de significations. Les partenaires de jeu ont partagé les expériences individuelles autant dans l'accord des intentions du projet de construction que dans les décisions et dans les actions sur le matériel et sur le choix du matériel. Ils partagèrent également leur point de vue sur l'organisation et l'agencement du matériel pour la représentation d'objets ou d'événements et dans la négociation des rôles sur la tâche de production à réaliser comme sur l'évaluation des résultats et des actions. De plus, ces énoncés regroupent un ensemble d'intérêts communs aux enfants de ce groupe d'âge traduisant leur connaissance de l'environnement et répondant ainsi à certaines attentes des observateurs de l'activité de construction. 
Évidemment, la fréquence et la précision des énoncés verbaux quant aux actions à poser et aux rôles à distribuer et à assumer sont dépendantes des caractéristiques de la situation et en particulier de la nature des objets, de l'intention retenue du projet de construction, des compétences des sujets, des relations interpersonnelles et, finalement, des expériences communes aux enfants.

Avec le matériel bloc que nous qualifions de matériel ouvert (matériel qui offre une multitude de possibilités de transformations), il devient donc possible pour les enfants de rejoindre une diversité de fonctions. La communication verbale en est une qui joue un rôle important dans l'activité de construction, puisqu'elle permet l'actualisation d'un projet pouvant se réaliser individuellement, à deux, trois ou quatre enfants.

Cette communication verbale fera l'objet d'une analyse plus approfondie lorsque nous aborderons la troisième question de recherche de cette étude qui porte sur les interactions sociales.

\section{Thème 2 - Les données relatives aux produits de l'activité}

À partir des observations informelles et quotidiennes, on se rend compte qu'au coin bloc, tout peut se produire! L'enfant est devant d'infinies possibilités qu'il peut exploiter selon ses expériences. Lorsqu'il arrive dans cet espace, il a d'abord à créer son cadre de référence et son contexte de jeu. La création de contexte est tributaire des productions que l'enfant réalise. C'est d'abord et avant tout le fruit de son activité de construction qui donne naissance à une activité de jeu symbolique. Ainsi dans cet espace, les enfants ont donc la possibilité d'inventer une variété de contextes.

Cuffaro (1984), dans une réflexion théorique, s'intéresse au jeu dramatique en relation avec les blocs de construction dans une situation de groupe. Le matériel bloc devrait permettre, à son sens, d'explorer non seulement le jeu de l'enfant, mais la nature du médium et voir comment celui-ci affecte et influence l'activité de l'enfant. Elle analyse donc la nature du matériel bloc, ses caractéristiques, ses opportunités et la contribution potentielle de ce matériel au jeu dramatique.

Elle définit le jeu dramatique comme une activité. L'enfant, à partir du matériel bloc, élabore un objet, c'est-à-dire une structure. Il dote cette structure d'une fonction 
imaginée à partir de ses représentations, ses réflexions, ses expériences, ses fantaisies et ses diverses connaissances. La structure est donc une représentation tangible de ses idées personnelles, de ses désirs, de ses sentiments et de ses émotions et, dans cette forme symbolique, les structures servent de stimuli pour élargir l'activité et l'imaginaire.

Cuffaro identifie donc trois tâches spécifiques que l'enfant doit accomplir en utilisant les blocs pour le jeu dramatique: 1) créer le contexte pour le jeu plutôt que de le trouver créé au départ de l'activité; 2) négocier avec la réalité et les données en transposant ses idées à l'aide du médium; 3) graduellement, se distancier de soi vers une symbolisation de soi dans le jeu.

Ces trois tâches sont identifiées spécifiquement lorsqu'il y a utilisation des blocs pour le jeu dramatique. Bien que nous acceptons cette identification de tâches de Cuffaro, il nous semble qu'il serait opportun de les regarder d'abord sous l'angle de l'activité de construction car, selon nos observations, cette activité est le pivot et le point de départ de l'activité de jeu symbolique. C'est pourquoi nous analyserons ces trois tâches également dans l'activité de construction afin d'en dégager les stratégies émergentes déployées par l'enfant pour rendre possible la réalisation de diverses productions.

\section{- Première tâche: création d'un contexte}

La première de ces tâches concerne le contexte du jeu et met l'emphase sur les structures. Dans cette étude, les structures sont nommées productions.

Dans l'ensemble des recherches recensées sur le matériel bloc vu dans une perspective développementale, peu d'entre elles font mention du type de productions élaborées par les enfants. Dodge (1979a) a identifié certains cadres habituellement élaborés par les enfants pour le jeu dramatique: zoo, bateau, lance, fusil, missiles, ferme, pièces d'une maison, aéroport, garage, stationnement, tunnel, restaurant, caserne de pompiers, autoroute, station ou poste de police, etc.

Pour sa part, Johnson (1974), dans son étude sur les différents procédés de construction utilisés pour situer les enfants dans des stades d'évolution de la construction à partir du matériel bloc, mentionne quelques productions nommées par les enfants: décoration, maison, train, lit, parc, bébé avion avec la maman avion, cheval, défilé, hôpital, 
escaliers, etc. Elle ajoute également que le groupe de 5 ans construit: des systèmes de chemin de fer, des stations, des engins, des tours de contrôle, etc.

À partir d'une perspective sociale, on note que les types de productions réalisées par les enfants à partir du matériel bloc sont considérés comme objet de jeu. Ce point de vue social du jouet met en évidence deux dimensions importantes de l'objet de jeu: la dimension matérielle et la dimension symbolique. Les types de productions observées dans l'aire des blocs interrogent à la fois ces deux dimensions.

Dans sa dimension matérielle, les blocs de Caroline Pratt, utilisés dans cette étude, proposent différentes formes qui invitent d'abord à une activité de construction. Sans l'intervention de l'enfant, les blocs eux-mêmes demeurent des formes simples et sans représentation qui, par contre, orientent déjà dans leur matérialité le genre de productions que les enfants peuvent réaliser.

Dans sa dimension symbolique, l'intervention de l'enfant avec ce matériel donne un autre sens. En effet, l'enfant, à partir des différentes formes de blocs, peut représenter autant d'univers familiers que d'univers imaginaires, des univers déjà imprégnés dans la matérialité des blocs eux-mêmes.

Dans notre étude, tous les enfants (sauf deux cas) nomment d'abord leurs intentions de projet de construction et ce, dès les premières minutes de l'activité. Par la suite, tout au long de l'activité, ils nomment les nouveaux projets qui s'y greffent et les différentes modifications apportées au projet initial ou aux nouveaux projets. Ces différents projets annoncés sont négociés si deux enfants et plus deviennent partenaires de jeu. Ces négociations verbales peuvent être acceptées, refusées ou modifiées, selon une variété de stratégies que les enfants utilisent dans diverses expériences. Ces diverses stratégies seront démontrées dans l'analyse de la troisième question de recherche. Ces deux orientations théoriques (développementale et sociale) proposent quelques pistes de productions réalisées par les enfants. Ces pistes demeurent toutefois très peu exhaustives sur les divers possibles de productions à partir du matériel bloc. Considérant l'importance et l'influence du matériel sur les possibilités de productions, il nous est apparu fondamental d'investiguer les diverses voies d'exploitation de ce matériel en fonction des types de productions réalisées par les enfants, afin peut-être de dégager un certain profil de ces types de productions. 
De toutes les productions réalisées durant les neuf séances d'observation, il a été possible d'identifier quatre grandes catégories: 1) des productions représentant différents genres d'édifices; 2) des moyens de transport; 3) des systèmes routiers simples et complexes et 4) une dernière catégorie appelée "divers" regroupant des productions autres que celles mentionnées dans les autres catégories. Ces catégories ou ces contextes élaborés par les enfants sont présentés au tableau 4.2.

La prise en compte de ces différentes catégories de productions représentant l'ensemble des projets annoncés et initiés par les enfants lors de l'étude permet de dégager une constante commune dans l'élaboration de projets initiaux qui constituent la trame générale, un contexte de base propre à l'activité de construction. Ce contexte matériel permet et oriente l'activité de construction d'abord.

Par la suite, il pourra déclencher une activité de jeu symbolique. Selon nos données de recherche, ce contexte de base créé par l'enfant est d'abord et avant tout un contexte engendré par l'activité de construction. Dans le cadre de cette recherche, il est identifié comme étant le projet global.

\section{- Deuxième tâche - Transposition des idées/négociation avec la réalité}

La deuxième tâche identifiée par Cuffaro (1984) concerne la négociation que l'enfant amorce avec la réalité et les données afin de pouvoir transposer ses idées de représentation à l'aide du matériel bloc. Par exemple, l'enfant annonce son intention de construire une maison. Pour chaque enfant, le concept de maison fait référence à un ensemble d'idées constituant la représentation de la maison. Ce que l'enfant retient de ses idées est pour lui la plus grande signification qu'il attribue au concept de maison. Cette représentation de la maison est à la fois un amalgame d'informations sélectionnées par l'enfant et le rapport qu'il établit entre lui et l'objet à représenter et les sentiments et les émotions relatifs à cette représentation.

Ainsi, pour un enfant, le concept de maison peut être essentiellement représenté par la construction de la cuisine, de la salle de bain, du salon ou toute autre pièce de la maison, et pour un autre enfant, ce concept peut être représenté par une enclosure avec des fenêtres et des portes. 
Tableau 4.2

Les différentes catégories regroupant l'ensemble des productions élaborées par les enfants

\begin{tabular}{|c|c|c|c|}
\hline \multirow[t]{4}{*}{$\begin{array}{l}\text { PREMIÈRE CATÉGORIE: } \\
\text { GENRES D'ÉDIFICES }\end{array}$} & Maisons & \multicolumn{2}{|c|}{$\begin{array}{l}\text { Maison avec beaucoup d'étages } \\
\text { Maison à trois étages } \\
\text { Un village de maisons } \\
\text { Maison pour rentrer dedans } \\
\text { Maison-château } \\
\text { Maison hantée } \\
\end{array}$} \\
\hline & Châteaux & \multicolumn{2}{|c|}{$\begin{array}{l}\text { Château de fantômes } \\
\text { Château-bateau } \\
\text { Château de voitures } \\
\text { Château d'armée } \\
\text { Château pour les géants } \\
\text { Château de princesse } \\
\text { Château d'animaux } \\
\text { Château de sirène } \\
\end{array}$} \\
\hline & Garages & \multicolumn{2}{|c|}{\begin{tabular}{|l|} 
Garage de pompiers \\
\end{tabular}} \\
\hline & Autres & $\begin{array}{l}\text { Tour de pompiers } \\
\text { Grosse base } \\
\text { Édifice } \\
\text { Royaume } \\
\text { Poste de police } \\
\text { Édifice pour les autc } \\
\text { Fourrière d'animaux } \\
\text { Cage à voleurs de pe } \\
\text { Caverne } \\
\text { Ferme } \\
\end{array}$ & $\begin{array}{l}\text { Zoo } \\
\text { Cabane } \\
\text { Bâtisse } \\
\\
\text { les }\end{array}$ \\
\hline \multirow[t]{3}{*}{$\begin{array}{l}\text { DEUXIÈME CATÉGORIE: } \\
\text { MOYENS DE TRANSPORT }\end{array}$} & Terre $\rightarrow$ autos & \multicolumn{2}{|c|}{$\begin{array}{l}\text { Auto de sirène } \\
\text { Auto pour embarquer à quatre }\end{array}$} \\
\hline & Mer $\rightarrow$ bateaux & $\begin{array}{l}\text { Bateau de dynamite } \\
\text { Bateau à moteur } \\
\text { Bateau de pirates } \\
\text { Bateau de polices } \\
\text { Bateau de canons } \\
\text { Bateau d'espace } \\
\end{array}$ & $\begin{array}{l}\text { Bateau de voleurs } \\
\text { Bateau de secours } \\
\text { Bateau qui vole } \\
\text { Traversier d'autobus } \\
\text { Sous-marin }\end{array}$ \\
\hline & Air & $\begin{array}{l}\text { Avion de police } \\
\text { Fusée du Japon } \\
\text { Vaisseau } \\
\end{array}$ & \\
\hline $\begin{array}{l}\text { TROISIEMME CATÉGORIE: } \\
\text { SYSTĖMES ROUTIERS }\end{array}$ & $\begin{array}{l}\text { Chemin } \\
\text { Rue } \\
\text { Route } \\
\text { Piste pour faire rouler } \\
\text { Autoroute } \\
\text { Piste de course } \\
\text { Gros pont } \\
\end{array}$ & & \\
\hline $\begin{array}{l}\text { QUATRIÈME CATÉGORIE: } \\
\text { DIVERS }\end{array}$ & $\begin{array}{l}\text { Piscine } \\
\text { Labyrinthes } \\
\text { Parcs d'autos } \\
\text { Barrage }\end{array}$ & & \\
\hline
\end{tabular}


Cependant, peu importe la voie empruntée pour représenter son idée, la tâche de l'enfant dans l'activité de construction consiste à transposer son idée/concept intériorisé en une représentation extériorisée. L'enfant doit donc négocier entre la représentation interne et la représentation externe qu'il opérationnalise par le biais du matériel bloc. Il doit donc tenir compte à la fois d'une négociation matérielle, c'est-à-dire le matériau, les formes, les longueurs, les largeurs des blocs pour construire sa représentation, et d'une négociation plus abstraite afin de sélectionner les caractéristiques les plus significatives pour traduire cette idée. Ainsi, l'enfant doit adapter son intention de représentation aux différentes possibilités que lui propose le matériel bloc, afin de trouver la meilleure façon de traduire son idée.

Cette deuxième tâche entraîne l'enfant à découvrir différentes stratégies pour opérationnaliser son idée, en production tangible et observable. Après avoir créé le contexte de base de son activité de construction, l'analyse des données montre que l'enfant initie d'autres projets de construction que nous identifions comme des projets secondaires. Ceux-ci permettent de raffiner son projet global en élaborant des productions complémentaires. Chaque contexte ainsi élaboré entraîne l'enfant dans la réalisation de structures diverses qui viennent témoigner de ses connaissances à propos du contexte de base.

Dans cette étude, l'observation et l'analyse de l'élaboration de structures appartenant à des projets secondaires et complémentaires permettent d'identifier deux types de transformations utilisés par l'enfant: des transformations matérielles et des transformations symboliques. Lorsque l'enfant utilise les blocs pour les agencer et les organiser entre eux en structure pour une production particulière, nous parlons de transformations matérielles. Cependant, lorsque l'enfant utilise un bloc et lui attribue une signification ponctuelle en fonction de ses besoins, sans pour autant effectuer une transformation matérielle, nous parlons de transformations symboliques. Par exemple, quand un bloc se transforme en cassette.

Les différents projets secondaires et complémentaires émergent de chacun des contextes de base. Chaque grande catégorie de types de productions représente autant de contextes globaux, et chacun de ces contextes globaux entraîne l'élaboration d'un certain nombre de projets secondaires et complémentaires. 
Examinons donc des exemples tirés de deux séances différentes qui montrent l'élaboration de projets secondaires et complémentaires. Comme premier exemple, les enfants 3 et 4 de la séance 5 ont d'abord élaboré un contexte de base, la maison. Par la suite, ils ont construit une douzaine de projets secondaires et complémentaires pour raffiner le projet global du début. Ils ont apporté un certain nombre de transformations matérielles pour construire des barrages, des labyrinthes, des chemins, un carré de sable, une salle de bain, des bancs et des bancs de secours. Simultanément, ils ont réalisé des transformations symboliques avec des blocs de formes variées pour signifier une porte patio (Y), un lac, une cheminée (cylindre), un marteau (demi-cercle romain) et un clou (triangle).

Comme deuxième exemple, l'enfant 3 de la séance 7 a d'abord créé son contexte de base en annonçant un poste de police. Par la suite, différents projets secondaires ont complété et raffiné ce contexte de base tels que les murs, une entrée, un parking, un enclos, une clôture et simultanément, l'enfant a utilisé des blocs pour signifier d'autres éléments de son projet global: un bonhomme de police (carrefour), un radar (une colonne), une girouette (panneau de signalisation).

Ces deux exemples renseignent sur les diverses modalités de fonctionnement des enfants au coin bloc. L'ensemble des projets globaux et secondaires est présenté à l'appendice A-3.

\section{- Troisième tâche - Processus de distanciation}

La troisième tâche concerne le processus évolutif de l'enfant à se distancier de lui vers une symbolisation de soi dans le jeu. En d'autres mots, la distanciation, c'est la capacité de l'enfant à se séparer de lui-même et simultanément à être à l'intérieur de luimême.

Cuffaro (1984) présente trois stades d'évolution de ce processus de distanciation dans le développement du jeu dramatique avec les blocs. Vers 5-6 ans, l'enfant, qui, au début de son jeu dramatique, participait directement dans les divers rôles joués, devient capable de participer à ces rôles dans un soi symbolisé à travers des figurines-personnages. L'enfant est alors capable de se distancier de lui-même afin de comprendre les différents cadres de référence et les points de vue, autres que les siens. Il commence à se décentrer de son propre point de vue pour voir et comprendre le point de vue des autres. 
La participation directe et l'engagement du soi qui caractérisent les premier et deuxième stades sont abandonnés au profit d'un soi symbolisé à partir duquel sentiments et émotions sont projetés.

Cuffaro (1984), dans cette troisième tâche, identifie l'évolution de ce processus seulement dans le développement du jeu dramatique avec les blocs. Dans la présente étude, selon les observations réalisées au coin bloc, il semble bien que ce processus de distanciation s'effectue également dans l'évolution de l'activité de construction proprement dite, et, il se manifesterait de deux façons dans l'orientation des dimensions des structures élaborées. Effectivement, l'enfant décide de construire une structure proportionnelle à sa taille, ce qui peut suggérer que l'enfant s'investira lui-même en tant qu'acteur, ou bien il construit des structures pouvant servir à l'élaboration de scénarios autour de figurines-personnages ou animaux ou à des moyens de transport-jouet.

L'investigation de cet élément indicateur dans l'activité de l'enfant avec le matériel a permis, dans une première analyse, d'identifier les types de productions qui ont été élaborés aux dimensions de la taille des enfants et aux dimensions de figurines-personnages et animaux. Par la suite, une deuxième analyse a répertorié tous les énoncés verbaux de chacun des enfants qui ont réalisé des structures à leur taille afin de dégager les intentions quant à leur souci d'élaborer ou non des structures à grande dimension.

Les neuf séances d'observation impliquent la participation de 36 enfants. De ce nombre, 50\% (19) ont réalisé des structures aux dimensions de leur taille. Ces différentes productions sont observées dans les séances 2-3-4-5-7-8-9 et deux seulement, impliquant la participation de huit enfants, séances 1-6, ont engendré des types de productions à la taille des figurines. Dix-sept autres enfants (environ 50\%) ont réalisé des structures aux dimensions de la taille des figurines. Ces différentes productions sont observées dans les séances 1-3-4-6-7-8-9. Deux seulement impliquent la participation de huit enfants, séances $2-5$, et engendrent des productions à la taille des enfants.

Donc, dans l'activité de construction, à partir du matériel bloc, la moitié des productions est réalisée à la taille des enfants et une autre moitié à la taille des figurines.

$\mathrm{Si}$ on observe la façon dont les dyades, triades ou groupes de quatre enfants se constituent et les mêmes enfants se retrouvant seuls, il semble bien que l'ensemble des 
enfants chevauchent dans l'un ou l'autre de ces modes de construction autant pour des projets individuels que pour des projets collectifs. Un seul de ces enfants a toujours travaillé seul et a élaboré des productions de petites tailles. Les autres enfants qui s'associent dans la plupart des séances avec un, deux ou trois partenaires (huit séances) ou qui travaillent seuls (quatre séances) utilisent tantôt un mode de construction aux dimensions de leur taille et tantôt un mode de construction aux dimensions de la taille des miniatures.

Ces données indiquent donc un mode de construction mettant en œuvre l'élaboration de deux types de structures, chacun engendrant une implication et un investissement différents de la part de l'enfant.

Une deuxième analyse de cet indicateur relatif à la dimension des structures élaborées a permis de répertorier l'ensemble des énoncés verbaux associés aux deux types de structures.

Les données relatives à l'élaboration des structures à la dimension des figurines n'indiquent pas d'énoncés verbaux spécifiques traduisant l'intention des enfants quant à la taille du projet. Les enfants amorcent leur projet de construction, autant individuel que collectif, sans mentionner verbalement les dimensions de la structure. Le projet s'élabore, se poursuit, se termine ou s'abandonne. Les figurines-personnages, les animaux et les voitures-jouets sont introduits en cours d'élaboration et ainsi donnent naissance à différents scénarios plus ou moins élaborés.

Par contre, les données portant sur la réalisation d'un projet à la dimension de la taille des enfants se présentent tout autrement. En effet, tous les enfants qui s'orientent autant dans des projets individuels que collectifs annoncent verbalement leur intention d'élaborer des structures aux dimensions de leur taille. Ces énoncés verbaux sont formulés dès les premières minutes de l'activité dans leur projet global et, par la suite, dans différents projets secondaires et complémentaires.

Le tableau 4.3 présente quelques exemples d'énoncés verbaux de projets globaux et de projets secondaires et complémentaires portant sur des productions à la dimension de la taille des enfants. 
Tableau 4.3

Quelques exemples d'énoncés verbaux de projets globaux et de projets secondaires et complémentaires portant sur des structures à la dimension de la taille des enfants

\begin{tabular}{|c|c|c|c|c|}
\hline Séance & Temps & $\begin{array}{l}\text { Énoncés verbaux } \\
\text { Projet global }\end{array}$ & Temps & $\begin{array}{r}\text { Énoncés verbaux } \\
\text { Projets secondaires et complémentaires } \\
\end{array}$ \\
\hline SEA2-ENF1 & 03:09:19 & $\begin{array}{l}\text { Aïe ben nous autres, haye sais-tu qu'on l'a partie de l'a } \\
\text { nous autres, là on va faire des chemins comme ça, on } \\
\text { va rentrer dedans. }\end{array}$ & & \\
\hline SEA2-ENF3 & $\begin{array}{l}02: 28: 13 \\
03: 13: 13 \\
03: 27: 26\end{array}$ & $\begin{array}{l}\text { Wo, wo, moi pis Cédrick, on va faire une grande pour } \\
\text { rentrer dedans. } \\
\text { Ah, nous autres, on va rentrer dedans. Aïe } 4-2 \text {. } \\
\text { Vous pourrez pas rentrer dedans mais nous autres on va } \\
\text { pouvoir rentrer. }\end{array}$ & & \\
\hline SEA2-ENF4 & 01:39:02 & $\begin{array}{l}\text { On va faire une maison, une maison pour rentrer } \\
\text { dedans, hein, cette fois-là, on la fait grande, grande, } \\
\text { grande, grande la maison. }\end{array}$ & $\begin{array}{l}33: 05: 26 \\
34: 11: 18\end{array}$ & $\begin{array}{l}\text { Ça c'est notre lit (en se couchant). } \\
\text { Ah là là là, je vais dans la cuisine (en se déplaçant } \\
\text { vers la première partie de la construction). }\end{array}$ \\
\hline SEA3-ENF2 & & & $2: 41: 00$ & Je veux bâtir un gros banc. \\
\hline SEA3-ENF3 & $00: 32: 00$ & On va rentrer dedans. & $14: 11: 23$ & Mais nous on joue pas au bonhomme. \\
\hline SEA3-ENF4 & $\begin{array}{l}19: 07: 29 \\
19: 16: 10\end{array}$ & $\begin{array}{l}\text { Oh on embarque dans notre bateau. } \\
\text { On embarque dans notre bateau, nous autres là. }\end{array}$ & & \\
\hline SEA4-ENF2 & $01: 47: 29$ & $\begin{array}{l}\text { Moi je me fais un bateau, Jonathan. Veux-tu } \\
\text { embarquer dans mon bateau? }\end{array}$ & & \\
\hline SEA4-ENF3 & $08: 48: 20$ & Ça peut embarquer à trois, nous autres. & & \\
\hline SEA4-ENF4 & $\begin{array}{l}02: 45: 12 \\
05: 08: 24\end{array}$ & $\begin{array}{l}\text { Aie! On fait un plus grand bateau. } \\
\text { Ah oui! Ah oui! Oh moi je vais en faire un plus gros, } \\
\text { un bateau, ça va être le plus gros du monde entier } \\
\text { comme vous n'avez jamais vu de toute votre vie. }\end{array}$ & & \\
\hline SEA5-ENF1 & \begin{tabular}{|l|}
$02: 23: 03$ \\
$03: 13: 28$ \\
$07: 53: 22$ \\
$09: 01: 27$
\end{tabular} & $\begin{array}{l}\text { Ouais! Une grosse, une plus grosse que celle-là. } \\
\text { Non! C'est nous autres les bonhommes qui construit } \\
\text { notre maison. } \\
\text { Je vas rentrer dans ma maison, faut que j'aille, hein! } \\
\text { Oui mais. Ah oui! Oh! On peut coucher dedans (se } \\
\text { couche dans la construction). }\end{array}$ & $\begin{array}{l}11: 47: 08 \\
34: 27: 28\end{array}$ & $\begin{array}{l}\text { Je vas faire un autre banc, un banc, un banc (elle } \\
\text { s'assoit sur son banc). } \\
\text { Je vas jouer du piano (elle s'assoit sur son banc, met } \\
\text { ses pieds sur les pédales) 1-2-3-ta,ta,ta, 1-2-3-ta-ta-ta. }\end{array}$ \\
\hline SEA7-ENF1 & 14:00:18 & Ben sûr, mais faut rentrer dedans. & & \\
\hline SEA8-ENF3 & $\begin{array}{l}09: 58: 27 \\
18: 01: 23\end{array}$ & $\begin{array}{l}\text { Ouais, parce que, regarde quand on rentre, y nous en } \\
\text { manque pour rentrer, y nous en faut juste deux pour } \\
\text { rentrer. } \\
\ldots \text { je vas essayer pour voir si on est bien couché (se } \\
\text { couche recroquevillé sur le plancher de la construction). }\end{array}$ & & \\
\hline SEA8-ENF4 & 17:59:07 & Oh! je vas essayer de me coucher. & & \\
\hline
\end{tabular}




\section{Thème 3 - Les données relatives au temps consacré à l'activité de construction}

\section{- $\quad$ Fonctionnement de la classe maternelle}

Avant d'aborder les données inhérentes au temps consacré à l'activité de construction dans l'ensemble des études recensées et plus particulièrement dans cette étude, il est important de décrire les modalités de fonctionnement de la classe maternelle où s'est déroulée la cueillette des données.

Dans son mode de fonctionnement quotidien, la période d'ateliers de cette classe constitue le moment privilégié où l'enfant, à partir d'un tableau de programmation présentant l'ensemble des coins effectifs pour cette période, a la possibilité de choisir les activités qu'il désire réaliser pendant la période d'ateliers. Cette période dure une heure et se situe immédiatement après la causerie, première activité de groupe à l'arrivée des enfants.

Durant la période d'ateliers, chaque enfant en choisit un, en plaçant l'étiquette de son nom dans les espaces disponibles pour chaque atelier. Le nombre de participants dans un atelier varie en fonction de l'espace physique de l'aire et de la tâche à réaliser. Ainsi, au coin bloc, quatre enfants peuvent se retrouver simultanément dans cet espace de jeu. Habituellement, les règles de fonctionnement de cette classe établissent la possibilité de choisir un maximum de trois ateliers pendant une période d'une heure.

Cependant, pour fins d'observations et d'analyses, deux changements sont apportés au fonctionnement déjà établi.

Le premier changement porte sur l'exigence d'une présence simultanée de deux garçons et deux filles. Ce changement s'appuie sur différentes données de recherche qui considèrent traditionnellement le coin bloc comme un territoire de garçons. Il est aussi soutenu par les études qui indiquent que cette activité est prioritairement et majoritairement choisie par les garçons; ainsi que par d'autres résultats de recherche indiquant qu'à possibilité égale, les filles choisissent également le coin bloc. Donc, en déterminant ce changement, il devenait possible d'observer l'activité des filles et des garçons en même temps avec le matériel bloc. 
Le deuxième changement prolonge la durée de l'atelier à une heure. Ceci permet, sur le plan technique, d'observer les mêmes enfants et non pas une multitude d'enfants susceptibles de choisir cet atelier et de n'y rester que pour une période de très courte durée. Par la suite, sur le plan méthodologique, cette modification permet d'observer une activité plus élaborée, ou du moins plus spontanée et moins régie par le temps, c'est-àdire non limitée par sa durée comme dans la plupart des recherches sur les blocs où l'observation se réalise sur une période de 20 minutes à intervalles de cinq minutes.

Cette période plus longue permet l'observation des modes d'organisation et d'élaboration de l'activité et l'observation des comportements et des stratégies associés aux différentes activités suscitées par le matériel bloc. Ce choix, quant à la durée de l'observation, est également soutenu par différentes réflexions qui mettent en évidence la durée de l'activité / l'âge des enfants / la qualité de l'investissement / la qualité des productions / les processus / les stratégies.

\section{- Observation de la répartition du temps consacré à l'activité de construction et à l'activité de jeu symbolique}

Les données de recherche antérieures démontrent que ce sont les études sur les stéréotypes sexistes des jeunes enfants utilisant les blocs qui se sont préoccupées du choix de ce matériel de jeu et du temps consacré à l'activité de l'enfant avec ce matériel.

Dans l'ensemble, ces données révèlent que les garçons dominent l'aire des blocs (Sprung, 1978), qu'ils tendent à s'impliquer plus activement et physiquement (Robinson, 1977), qu'ils choisissent de préférence les blocs ( $54,5 \%$ garçons/23,1\% filles) à d'autres activités et que finalement, les garçons dépensent plus de temps à cette activité que les filles ([Clark, Wyon and Richards, 1979; Coates, Lord ans Jakabovics, 1975; Sears, Vance and McCall, 1934; Rubin, 1977) voir: Varma, 1980; Beeson et Williams, 1980]; Smith, 1977).

Cependant, l'étude de Varma (1980) démontre que lorsque les filles ont la même possibilité d'accès et qu'elles sont encouragées à jouer avec ce matériel, elles choisissent autant cette activité que les garçons et le temps dépensé à cette activité augmente significativement. 
Les données quant à la répartition du temps consacré à l'activité de construction originent de l'analyse de deux éléments indicateurs: le temps de réalisation, le temps total de la séance de jeu et la répartition du temps de cette séance.

La période d'ateliers dure approximativement une heure. Les premières minutes sont utilisées pour faire le choix d'ateliers et les dernières minutes de la séance sont consacrées pour le rangement du matériel puisqu'à l'aire des blocs, cette période de rangement est plus longue (cinq à dix minutes); la période de jeu réel est donc approximativement de 45 minutes.

\section{- Modalités d'analyse}

Pour chaque séance d'observation, la durée totale de la séance a été notée. Par la suite, nous avons relevé le temps consacré à l'activité de construction proprement dite et le temps consacré à l'activité de jeu symbolique et ce, pour chacun des quatre enfants d'une même séance. Cette répartition permet de mettre en évidence le temps approximatif consacré à chaque type d'activités. La moyenne a été calculée pour chaque séance et ce, pour l'une ou l'autre des deux activités et finalement, la moyenne des moyennes a été effectuée. Le tableau 4.4 permet de visualiser globalement la répartition du temps pour l'activité de construction et pour l'activité de jeu symbolique.

D'une façon générale, la lecture de ces données révèle que les enfants ont consacré $59 \%$ du temps à une activité de construction et $39,5 \%$ du temps à une activité de jeu symbolique.

Dans l'ensemble de ces séances, l'activité de construction est l'activité de base. Les enfants commencent d'abord par la création d'un contexte global autour duquel viennent se greffer divers projets complémentaires et secondaires. Une fois ce contexte créé et les diverses productions réalisées, les enfants amorcent une activité de jeu symbolique qui émerge de la création du contexte. Dans ces séances, l'activité de construction constitue la première activité des enfants de laquelle sont produites différentes structures qui orientent une activité de jeu symbolique. De ces neuf séances, seule la séance 1 présente un enfant dont l'activité de jeu symbolique est la seule activité durant toute la séance d'observation. 
Tableau 4.4

La moyenne du temps consacré à l'activité de construction et l'activité de jeu symbolique

\begin{tabular}{|l|c|c|}
\hline \multicolumn{1}{|c|}{ Séance } & $\begin{array}{c}\text { Moyenne du temps } \\
\text { consacré à } \\
\text { l'activité de } \\
\text { construction }\end{array}$ & $\begin{array}{c}\text { Moyenne du temps } \\
\text { consacré à } \\
\text { l'activité de jeu } \\
\text { symbolique }\end{array}$ \\
\hline Séance 1 & $52 \%$ & $43 \%$ \\
\hline Séance 2 & $73 \%$ & $22,5 \%$ \\
\hline Séance 3 & $76 \%$ & $24 \%$ \\
\hline Séance 4 & $53 \%$ & $62 \%$ \\
\hline Séance 5 & $76 \%$ & $15,5 \%$ \\
\hline Séance 6 & $43 \%$ & $53 \%$ \\
\hline Séance 7 & $47 \%$ & $46 \%$ \\
\hline Séance 8 & $50 \%$ & $38 \%$ \\
\hline Séance 9 & $59 \%$ & $52 \%$ \\
\hline \hline $\begin{array}{l}\text { Moyenne des } \\
\text { moyenne }\end{array}$ & Filles Garçons & Filles Garçons \\
\hline & $70 \%$ 52\% & $23 \%$ \\
\hline
\end{tabular}

Dans d'autres séances $(3,4,6,7,8,9)$, on remarque que certains enfants passent plus de temps à l'activité de jeu symbolique (SEA3-ENF1: 43-57; SEA4-ENF4: 39,5-60; SEA4-ENF3-4: 46,5-53; SEA6-ENF: 44-56; SEA6-ENF3: 12-89,5; SEA7-ENF3-4: 3171; SEA8-ENF1-2: 23-76,5; SEA9-ENF3-4: 49-51, 35-65) qu'à l'activité de construction. Cependant, cette activité de jeu symbolique, dans toutes ces séances, est subordonnée à l'activité de construction. Cette activité de construction constitue le moteur de l'activité de jeu symbolique et une fois que cette activité est amorcée, l'empreinte de l'activité de construction se constitue dans le projet global et les projets complémentaires et secondaires. L'enfant s'engage dans une activité de jeu symbolique et ne recourt par la suite que très rarement à l'activité de construction.

L'analyse globale de ces séances indique que trois séances (SEA3, SEA7, SEA 9) impliquent une activité de jeu symbolique simultanée à une activité de construction. Dans cette situation, l'activité de construction constitue toujours le premier mouvement d'activités des enfants, c'est-à-dire que ce dernier a comme première préoccupation la création d'un contexte par le biais de l'élaboration d'une structure. Il n'a pas, à ce moment, le 
souci d'un scénario comme déclencheur de l'activité de construction. Mais, très rapidement, l'élaboration des structures entraîne des idées pour une activité de jeu symbolique qui, à son tour, entraîne d'autres idées d'élaboration de projets. Dans cette situation, l'activité de construction et l'activité de jeu symbolique sont concomitantes, l'une et l'autre deviennent déclencheurs soit de la réalisation de productions, soit d'amorce de rôle, de scénario et produisent des réactions qui assurent une évolution en simultanéité.

Les enfants, dans les premières minutes (SEA3-ENF3-4), initient un projet global de construction (un château) mais simultanément, ce projet global stimule l'élaboration de différents épisodes de jeu symbolique (05:06:29: des agents secrets; 07:35:28: le désert; 09:59:03: l'arche de Noé; 10:56:16: un festival de crocodingues; 15:19:25: fait semblant de nager; 19:38:06: une avalanche, un tremblement de terre, etc.) qui donnera naissance à d'autres projets globaux ou secondaires (bateau et auto). Ainsi dans cette séance, pendant les 34 premières minutes, on observe la présence d'une activité de construction de base et en simultanéité, l'amorce de nombreux épisodes d'activité de jeu symbolique qui déclenchent, à leur tour, différents projets secondaires et complémentaires de construction. Les huit dernières minutes de cette séance sont essentiellement constituées d'une activité de jeu symbolique, élaborée à partir du projet global et des projets secondaires et complémentaires.

Par contre, la séance 7 (SEA7-ENF3-4) se constitue autrement. Les 11 premières minutes sont consacrées à l'élaboration d'un projet global de construction (un poste de police), sans introduction d'épisodes d'activité de jeu symbolique. Par la suite, les 25 autres minutes sont consacrées à l'élaboration d'une activité de jeu symbolique avec simultanéité d'activité de construction. L'une et l'autre de ces activités s'influencent pour déclencher des projets de construction et des projets d'activité de jeu symbolique.

\section{Thème 4 - Les données relatives aux diverses formes de participation sociale}

Traditionnellement, la recherche sur les diverses formes de participation sociale dans l'activité ludique, identifie des niveaux hiérarchiques et linéaires de participation qui sollicitent des formes de participation allant des plus individualisées au plus hautement socialisées. Cette conception des diverses formes de participation sociale repose sur des bases théoriques piagétiennes qui considèrent la coopération comme l'étape ultime du dé- 
veloppement, donc comme l'aboutissement d'un long processus hiérarchique de socialisation.

Parten (1933) demeure à l'avant-scène dans l'identification de ces niveaux de participation sociale avec ses travaux réalisés auprès de jeunes enfants d'âge préscolaire. De ses nombreuses observations, elle a élaboré une échelle évolutive permettant de dégager des comportements sociaux spécifiques dans différents types d'activités de jeux. On y retrouve quatre niveaux de comportements sociaux. L'enfant s'engage d'abord dans un jeu solitaire, puis un jeu parallèle, par la suite son jeu devient associatif et finalement, coopératif.

Or, l'évolution des connaissances dans la quête incessante de la compréhension du développement de l'individu ainsi que les nouvelles voies de recherche générées par l'intérêt croissant de l'aspect social dans le développement cognitif, viennent perturber et requestionner l'ordre hiérarchique et linéaire établi. Ainsi la coopération, considérée jusqu'à maintenant en bout de ligne du processus de socialisation, s'infiltrerait tout au long du processus, où des modalités de participation sociale se construiraient dès le jeune âge. Ces modalités constitueraient des déterminants essentiels à un processus de coopération précoce qui implique des modes spécifiques d'interactions sociales (Ballion, Breauté, Rayna et Stamback, 1987; Garnier, 1987; Garnier et Brief, 1985; Garnier, La Tour, Ferraris, Quesnel, 1991; Trewarthen, 1977; Verba, 1987).

Dans ce dernier thème relatif à notre première question de recherche, seront considérées les diverses formes de participation sociale à partir des quatre niveaux identifiés par Parten: jeux solitaire, parallèle, associatif et coopératif.

Dans le fonctionnement quotidien de la classe maternelle retenue, le nombre de participants maximum au coin bloc est établi à quatre enfants. Cependant, même si les enfants se retrouvent à quatre dans cette aire de jeu, diverses formes de participation sociale apparaissent (tableau 4.5). 
Tableau 4.5

Les différents types de regroupement observés dans l'aire des blocs selon les formes de participation sociale pour chaque séance d'observation

\begin{tabular}{|r|c|c|c|c|}
\hline & $\begin{array}{r}\text { Jeu solitaire, } \\
\text { parallèle }\end{array}$ & \multicolumn{3}{|c|}{ Jeu associatif, coopératif } \\
$\begin{array}{r}\text { Formes de } \\
\text { partipation } \\
\text { sociale: } \\
\text { type de } \\
\text { regroupement } \\
\text { Séances }\end{array}$ & Seul & Dyade & Trois & Quatre \\
\hline SÉANCE 1 & 4 & & & \\
\hline SÉANCE 2 & & 2 & & \\
\hline SÉANCE 3 & 2 & 1 & & \\
\hline SÉANCE 4 & 2 & 1 & & \\
\hline SÉANCE 5 & & 2 & & \\
\hline SÉANCE 6 & 2 & 2 & & \\
\hline SÉANCE 7 & & 2 & & \\
\hline SÉANCE 8 & & & & \\
\hline SÉANCE 9 & & & & \\
\hline
\end{tabular}

De ces données, on remarque que dans la forme de participation sociale de jeu solitaire ou parallèle, des projets individuels se sont réalisés dans quatre séances (séances 1 , $3,4,6$ ) et qu'une seule séance (séance 1) a donné lieu à quatre projets individuels simultanés. Il est important de souligner que cette séance était la première de toute la série d'observations.

Dans ces différents projets individuels, un seul enfant, le même, a réalisé ses projets seuls avec très peu d'interactions avec les autres enfants de l'aire.

Les autres projets individuels ont été réalisés par différents enfants qui ont, lors d'autres séances d'observation, participé avec d'autres enfants à des projets collectifs. Cependant, les projets individuels se sont toutefois réalisés d'une manière parallèle avec les autres enfants de l'aire suscitant des modalités variées d'interactions sociales.

De plus, sept séances ont donné lieu à une forme de participation de types associatif et coopératif entre deux partenaires et finalement, une séance a suscité un projet coopératif 
entre quatre partenaires; aucune de ces séances n'a donné lieu à des projets coopératifs à trois partenaires.

Cette première catégorisation de participation sociale permet d'établir, dans un premier temps, les types de regroupement possibles entre enfants du même âge. Ainsi, le mode de regroupement en dyade apparaît comme étant le mode de participation sociale le plus fréquemment adopté par ces enfants. On remarque que 22 enfants se sont organisés de façon dyadique pour leur activité à l'aire des blocs. Le deuxième mode de participation utilisé est le mode solitaire et parallèle où dix enfants se sont affairés à des productions individuelles impliquant toutefois des modalités variées d'interactions sociales. Comme neuf de ces enfants, à d'autres moments d'observation, ont participé à des projets collectifs, il est possible de supposer que ces enfants possèdent un répertoire de compétences sociales, mais qui pour différentes raisons, peuvent très bien décider par goût, par besoin ou par tempérament, de choisir de jouer seuls. Ici le jeu solitaire ne traduit plus une forme moins mature du jeu social, mais peut-être bien un autre choix de mode d'organisation de l'activité ludique (Rubin, 1977).

Enfin, seulement une séance a impliqué un mode de participation sociale à quatre partenaires. Cette formation de quatre enfants constitue assurément une dynamique coopérative plus complexe impliquant des modes spécifiques d'interactions sociales qui permettent la viabilité autant sur un plan quantitatif que qualitatif de l'activité.

Cette première piste d'investigation, quant aux formes de participation sociale, n'est que la pointe de l'iceberg. Elle met en lumière, certes, les diverses formes de participation sociale engendrées par l'activité de l'enfant avec le matériel bloc, mais éveille à un ensemble de questionnements sur les modalités d'interactions sociales. Les interactions favoriseraient l'association de partenaires, l'élaboration de projets à un ou plusieurs, la viabilité du projet et la fin ou l'abandon du projet élaboré.

Ces modalités d'interactions sociales, en regard des différentes formes de participation sociale, feront l'objet d'une analyse plus raffinée lors de l'examen de la troisième question de recherche. En effet, les réponses à la première question de recherche n'épuisent pas l'analyse, car elles deviennent des éléments de base essentiels à la compréhension de la dynamique des interactions sociales générées par l'activité de construction et de jeu symbolique. 


\subsection{Deuxième question de recherche: les composantes structurales de l'activité de jeu symbolique à partir du matériel bloc}

La deuxième question de recherche aborde l'activité de jeu symbolique. Ce questionnement origine de deux sources. La première vient de l'observation des enfants au coin bloc. Dans cette activité de construction, l'observateur voit apparaître à certains moments de l'activité divers éléments de jeu symbolique, plus ou moins complexes et plus ou moins élaborés, tels que des bribes de scénario amorcées, des rôles joués par les enfants et des transformations symboliques du matériel bloc. Bref, un ensemble d'éléments susceptibles de refléter la présence d'un jeu symbolique.

La deuxième source en est une d'ordre théorique. En effet, l'ensemble des auteurs qui ont élaboré les stades d'évolution de la construction ont observé, chez les enfants de 5-6 ans, 1'apparition d'une forte impulsion au jeu dramatique à partir et autour des productions. Cependant, aucune spécificité de ce jeu avec le matériel bloc n'a été décrite explicitement. Il semblerait donc que l'on attribue à ce jeu dramatique les mêmes composantes structurales du jeu dramatique, habituellement observé dans les situations considérées comme génératrices de l'activité symbolique. Selon ces considérations et selon la nature de l'activité que le matériel bloc suscite chez l'enfant, il semble bien que ce matériel engendre d'abord, une activité de construction qui se traduit dans des productions constituant ainsi un contexte de base à cette activité. Ce contexte suscite par la suite une activité de jeu symbolique.

Ces considérations entraînent donc l'investigation des composantes structurales de l'activité de jeu symbolique à partir du matériel bloc, d'où la formulation de la deuxième question de recherche.

Quelles sont les composantes structurales de l'activité de jeu symbolique à partir du matériel bloc?

\subsubsection{Les modalités générales d'analyse}

Pour répondre à cette deuxième question de recherche, une première lecture du "verbatim" de chacun des enfants des neuf séances d'observation a été réalisée, afin d'identifier les épisodes de l'activité de jeu symbolique suscitées par le matériel bloc. 
L'identification des épisodes reflétant des éléments de jeu symbolique supposait que le relevé de l'ensemble de ces éléments devrait se réaliser seulement dans l'activité de jeu symbolique simultanée ou subordonnée à l'activité de construction. Cependant, la lecture du "verbatim" a révélé la présence d'un ensemble d'éléments du jeu symbolique à l'intérieur même de l'activité de construction. Nous avons alors reconsidéré la façon de mettre en forme les données relatives aux diverses composantes structurales et nous avons décidé d'examiner également la présence et la nature des composantes structurales du jeu symbolique dans l'activité de construction. Une fois ces épisodes identifiés, le contenu de chacun a été filtré, selon les cinq composantes structurales du jeu de faire-semblant, élaborées par l'ensemble des auteurs préoccupés par ce type de jeu.

Ces cinq composantes structurales constituent donc les cinq thèmes de réflexion retenus pour analyser la deuxième question de recherche. Alors, afin de déterminer s'il y a similitude ou spécificité de l'activité de jeu symbolique à partir du matériel bloc et s'il y a spécificité, quelles en sont les modalités pour la mise en œuvre des activités de jeu symbolique dans le contexte de l'aire des blocs?

\section{- Les unités d'analyse}

Les études de Verba (1990) sur la construction et le partage de significations dans les jeux de fiction entre enfants ont orienté et inspiré les lignes directrices pemettant ainsi d'identifier les épisodes significatifs. C'est pourquoi:

a) dans un premier temps, une analyse globale de chaque séance d'observation identifie les diverses formes de participation sociale (voir tableau 4.5);

b) par la suite, pour chaque forme de participation sociale, l'identification des épisodes se fait comme suit: chaque fois qu'une dynamique impliquant des éléments reflétant un jeu symbolique est rencontrée dans une observation, l'on recherche l'origine sociale ou cognitive de cette dynamique pour déterminer le point de départ de l'épisode; ensuite, celui-ci est délimité par sa fin, c'est-à-dire l'abandon ou la transformation du jeu; 
c) pour chaque épisode, les conduites successives des enfants concernant les actions, les objets utilisés ou singularisés, les comportements et les énoncés sont relevés. (Verba, 1990, p. 32-33).

Les unités d'analyse sont donc identifiées à partir de ces données. Chaque unité d'analyse constituant une action de faire-semblant ou un échange avec le partenaire à son propos a été considérée en fonction des cinq composantes structurales du jeu symbolique. Une unité d'analyse constitue, par conséquent, un apport sur le plan de la fiction et/ou présente une ou plusieurs significations dans la construction interactive.

Chaque composante structurale est d'abord présentée théoriquement afin d'identifier la nature et la fonction dans l'ensemble des épisodes. Par la suite, sont analysés l'enchâ̂nement et la réciprocité de chacune des composantes les unes sur les autres afin de bien cerner l'intégration et la synergie de ces diverses composantes pour la mise en œuvre de l'activité de construction et de l'activité de jeu symbolique.

\section{- Première composante structurale: la décontextualisation et la substitution d'objets}

Les recherches sur la décontextualisation et la substitution d'objets rattachent cette première propriété structurale au jeu symbolique solitaire.

La décontextualisation se réfère aux comportements de faire-semblant associés aux diverses expériences de vie (manger, dormir ou autres) qui se manifestent en dehors du contexte, ou à des circonstances qui entourent habituellement ces comportements (Almy et al., 1982; Fein, 1981; Rubin et al., 1984). Ces études sur le faire-semblant solitaire démontrent que les décontextualisations se développent dans des séquences de plus en plus complexes. Cependant, étant donné que la décontextualisation est une composante du jeu solitaire, seule la substitution d'objets est considérée dans le jeu symbolique social.

La substitution d'objets est l'une des composantes les plus étudiées par les chercheurs. Elle prend des formes de plus en plus sophistiquées et devient une composante significative sous l'angle du développement (Doyle, 1988). La substitution d'objets se rapporte à l'habileté à assimiler un objet à un autre dans le jeu de faire-semblant. Deux 
préoccupations majeures se dégagent de l'ensemble des études: l'identification de l'âge dans l'apparition des comportements de substitution et l'influence de la forme et de la fonction des objets utilisés sur les habiletés de transformation d'objets. Cette habileté à substituer commence dès 19 mois et augmente au cours des années préscolaires et dans les premières années scolaires (Rubin et al., 1984; Watson et Fischer, 1977). Certains chercheurs ont tracé, sur un continuum de développement, l'évolution de ce comportement de substitution des objets (Fein, 1975; Gowen, 1978; Wolfgang et al., 1981).

Par la suite, les chercheurs se sont interrogés sur l'influence du type d'objets utilisés sur le processus de transformation. Les résultats révèlent qu'un objet avec une fonction ambiguë ou considéré neutre, comme les blocs, est plus facile à substituer qu'un objet ayant une fonction clairement définie, devenant ainsi plus conflictuel à substituer, exemple une auto (Doyle, Connoly et Rivest, 1980; Jackowitz et Watson, 1980; Matthews, 1977; McLoyd, 1980). Dans ce cas, la transformation de l'objet par l'enfant demande d'attribuer une autre fonction à l'objet qui en possède déjà une, c'est pourquoi il est plus facile pour les enfants $(18,26,30,36$ et 42 mois) de faire semblant avec des objets dont la similarité avec l'objet réel est plus grande qu'avec des objets moins ressemblants (Elder et Pederson, 1978; Fein et Robertson, 1975; Jeffree et McConkey, 1976). Pour les petits, l'objet et sa fonction sont étroitement reliés, tandis que les plus âgés dissocient plus facilement la fonction de l'objet. Dans le cas d'un objet non ressemblant, l'enfant doit dépasser la signification de l'objet et construire une autre signification pour faire semblant. Lorsqu'il n'y a pas d'objet et qu'il n'y a qu'à générer l'action, l'enfant n'a pas l'obstacle d'une double signification, comme c'est le cas avec l'objet non ressemblant.

Entre trois et cinq ans, le faire-semblant est davantage lié à une notion, indépendant de la présence immédiate d'un objet matériel ou d'un objet aux fonctions semblables (Doyle, Doehring et De Lorimier, 1988). Cette aptitude à substituer se développe jusqu'à huit ans approximativement, au moment où les enfants deviennent capables de représenter des objets absents uniquement par des gestes (Overton et Jackson, 1973), préférant toutefois utiliser de plus en plus des objets réels lorsqu'ils sont disponibles (Connoly, Doyle et Ceschin, 1983). De plus, à mesure que les enfants vieillissent, l'utilisation des objets est de plus en plus appropriée, réaliste ou conforme aux modes de la vie adulte et à la fois, paradoxalement, les enfants peuvent utiliser un objet pour une tout autre action que 
sa fonction première. Cela démontre que le concept de notion devient plus important que les objets eux-mêmes (Garvey, 1977).

\section{- Deuxième composante structurale: le jeu social ritualisé}

Cette composante structurale du jeu se rattache à un jeu symbolique social. Elle concerne la relation du rôle entre les partenaires sociaux. Le faire-semblant ritualisé est gouverné par l'acceptation de ce qui apparaît être implicitement consenti sur le rôle de chacun des partenaires dans les séquences interactives (Garvey, 1974). Ce jeu social ritualisé se définit comme étant une des premières formes de comportements interactifs qui apparaît dans le jeu symbolique. Il devient de plus en plus complexe avec l'âge en progressant selon des stades d'évolution dans les interactions sociales.

Le premier stade est caractérisé par des comportements symétriques identiques, c'est-à-dire que le partenaire de jeu est probablement une copie exacte des effets physiques produits avec les jouets ou avec les objets par l'autre partenaire de jeu. Cette symétrie peut se répéter plusieurs fois dans l'épisode de jeu (Mueller et Lucas, 1975; Ross, Goldman et Hay, 1979; Vandell et Mueller, 1980).

Dans un stade plus avancé de l'interaction sociale, les partenaires se conduisent dans des voies particulières ritualisées dans lesquelles les actions sont intercoordonnées. Chaque partenaire définit son rôle en fonction du rôle de l'autre, un lanceur et un receveur (Mueller et Lucas, 1975).

Finalement, l'intercoordination des actions peut entraîner un renversement des rôles des partenaires, le lanceur devient le receveur et le receveur devient le lanceur. Cette intercoordination et cette réciprocité sont le dernier stade des interactions sociales des rôles ritualisés (Ross, Goldman et Hay, 1979).

Ce concept de jeu ritualisé des partenaires peut s'étendre aisément aux activités dramatiques des enfants de la maternelle et des écoles élémentaires. Le jeu dramatique des enfants plus vieux prend des accents distinctement ritualistes. L'observation sporadique des enfants peut donner l'impression d'un cadre de jeu fantaisiste et des actions très créatives, donc un faire-semblant moins répétitif et redondant que celui des enfants plus jeunes. Cependant, l'observation du jeu à thème fantaisiste des mêmes groupes d'enfants 
sur une base régulière révèle que les enfants prennent les mêmes rôles et poursuivent des actions similaires et mêmes identiques. Ainsi, plusieurs observations de jeu des mêmes groupes d'enfants peuvent indiquer l'existence de modes interactionnels plus complexes de faire-semblant qui sont néanmoins toujours ritualisés à divers degrés.

\section{- Troisième composante structurale: l'adoption de rôles}

Une troisième propriété structurale du faire-semblant social concerne les types de rôles adoptés par les participants au jeu dramatique. Cette composante a été l'objet de nombreuses études contemporaines.

Le jeu de rôle implique la personnification d'un rôle, c'est-à-dire la simulation de l'identité qui permet de reconnaître, de distinguer et d'apprivoiser les caractéristiques particulières et les tâches spécifiques d'un rôle donné. Il comporte également la mise en scène avec une ou plusieurs personnes, d'un thème commun, où les objets et l'environnement sont transformés (Doyle, 1988; Doyle, Doehring et DeLorimier, 1988; Fein, 1981).

Garvey est la figure majeure de cette ligne de recherche. Sans cesse préoccupée à découvrir les types de rôles dramatiques adoptés par les enfants, elle a identifié quatre types de rôles dramatiques joués par des enfants de 3 à 5 ans: les rôles fonctionnels (functional roles), les rôles relationnels (relational roles), les rôles de caractère (character roles) et les rôles périphériques (peripheral roles) (Garvey, 1977; Garvey et Berndt, 1977).

Les rôles fonctionnels sont définis comme des rôles qui sont organisés par un objet ou une action. Ils sont définis par la fonction et sont distincts des rôles familiaux et des rôles de caractère. Le chauffeur d'autobus et la cuisinière qui prépare le repas sont des rôles fonctionnels.

Les rôles relationnels font références aux rôles familiaux qui impliquent des relations complémentaires (mère-enfant, mari-femme). Ce sont les préférés des enfants et les scénarios centrés autour de ces rôles durent plus longtemps. Ils sont généralement joués d'une manière plus élaborée et dans des séquences plus cohérentes et ordonnées que tous les autres types de rôles. Ce sont les premiers rôles joués par les enfants, ils représentent les personnes de leur environnement immédiat. Les enfants construisent les séquences à 
partir d'une idée d'ensemble des rôles et des actions. Ces rôles relationnels ne sont pas des copies exactes des caractères et des actions des personnages joués. Ils peuvent aussi coïncider avec des rôles fonctionnels. Les rôles relationnels sont les plus fréquemment joués par les enfants (Garvey et Berdnt, 1977, Matthews, 1977).

Les rôles de caractère peuvent être regroupés sous deux catégories: les rôles stéréotypés et les rôles de fiction. Les rôles de pompier, policier, cow-boy et médecin sont considérés comme des rôles stéréotypés. Les rôles de fiction eux, sont issus, pour leur part, des histoires, de la télévision ou de la tradition orale. Ainsi, les enfants jouent des personnages comme le Père Noël, Batman et Cendrillon. L'observation des jeux de fiction révèle que les enfants parlent davantage des personnages fictifs qu'ils ne les jouent.

Les rôles périphériques sont ceux que les enfants discutent mais dont ils n'assument jamais l'identité. C'est en fait un troisième personnage réel ou imaginaire qui n'entre jamais dans l'espace de jeu proprement dit.

D'autres études sur les rôles adoptés par les enfants se sont préoccupées davantage de la structure interpersonnelle du jeu de rôles. Il s'en dégage que les enfants plus jeunes (trois ans) jouent des rôles différenciés et complémentaires, sans toutefois développer ce rôle en référence avec les autres rôles. Les enfants plus vieux (cinq ans) développent eux, des rôles complémentaires et intégratifs à travers des comportements réciproques et appropriés, liés à l'activité de chaque autre joueur (Fein et Stork, 1984; Garvey et Berdnt, 1977; Iwanaga, 1973; Watson et Fischer, 1980).

Finalement, même si peu de recherches ont été réalisées sur les jeux de rôles à l'âge scolaire (Connoly, Doyle et Ceschin, 1983), il semblerait aussi qu'avec l'âge, la cohésion du faire-semblant irait en s'accroissant, c'est-à-dire qu'elle serait plus coordonnée quant au thème et au comportement avec le partenaire (Gönçü, 1987b; Gönçü et Kessel, 1988).

- Quatrième composante structurale: le jeu fantaisiste thématique / le jeu sociodramatique

Une autre composante structurale du faire-semblant social concerne la distinction entre le jeu fantaisiste thématique et le jeu sociodramatique (Saltz, Dixon et Johnson, 1977; Saltz et Johnson, 1974; Saltz et Brodie, 1982). 
Dans un cadre d'interprétation vygotskien, les chercheurs préoccupés par cette différenciation indiquent que le jeu fantaisiste thématique, dans lequel les enfants jouent des rôles fantaisistes, solliciterait une forme plus mature du groupe de faire-semblant que le jeu sociodramatique. Distancié de leur expérience quotidienne et familière, ce type de jeu invite à un niveau de représentation imaginaire et symbolique plus élevé que le niveau de représentation provoqué par des jeux familiaux et relationnels, considérés comme les premières formes de groupe de faire-semblant (Garvey et Berndt, 1977; Mattews, 1977; Saltz et al., 1977). Durant les années préscolaires, les enfants seraient donc plus emprunts à jouer des rôles imitatifs et relationnels (Johnson, Ershler et Bell, 1980; Johnson et Ershler, 1981).

\section{- Cinquième composante structurale: la structuration du jeu}

La dernière composante structurale du jeu symbolique social est la communication qualifiée de métacommunication dans les références anglo-saxonnes et de métaludique ou de communication à propos du jeu dans celles de langue française. Du côté anglo-saxon, Bateson $(1955,1956)$ apparaît comme l'auteur le plus influent dans ce domaine de recherche.

Dans le jeu symbolique solitaire, le joueur décide à sa guise du contenu et du déroulement du jeu au gré de sa fantaisie. Il varie le thème, le scénario, les rôles et les accessoires. Il demeure le seul meneur de jeu, le seul acteur et le seul scénariste.

Dans le jeu symbolique social, chaque joueur doit maintenant tenir compte de l'autre dans le contenu et le déroulement du jeu. La coordination des partenaires n'est rendue possible que grâce aux différentes stratégies communicatives que l'un et l'autre utilisent dans la situation de faire-semblant. Ces stratégies établissent un cadre commun de référence dans lequel s'effectuent diverses transformations symboliques et matérielles et se réalisent divers échanges entre les partenaires.

Ainsi, la métacommunication met en évidence les actes de communication qui sont considérés comme centraux dans la mise en place et dans l'élaboration des jeux. Ces actes de communication présentent des fonctions différentes et contribuent à donner des informations sur le contenu et l'objet de la conversation (thème du jeu, rôles, accessoires, 
décors) mais également, à qualifier la nature de la relation interpersonnelle (accord, désaccord, valorisation, double injonction) (Bonica, 1990).

Par la suite, les recherches sur le partage des significations (Mussatti, 1983; Verba, 1985) viennent raffiner certains aspects de la métacommunication, en étudiant les processus de constitution d'un cadre de référence partagé qui assure le déroulement cohérent des jeux interactifs et en questionnant la façon dont les interactions entre enfants au cours du jeu amènent le partage.

La métacommunication signifie donc tout message facilitant l'interprétation des paroles et gestes de l'autre ou établissant les règles de comportement dans un contexte de jeu précis (Doyle, Doehring et DeLorimier, 1988; McLyod, 1980). Ce type de communication regroupe les énoncés concernant le contenu, le déroulement et le partage proprement dit du jeu (Fein, 1981; Garvey, 1982; Giffin, 1984, Verba, 1990).

La métacommunication est indispensable pour partager les expériences individuelles, négocier les thèmes et les rôles, choisir le décor et les accessoires, évaluer les performances, etc.

Le jeu symbolique social est donc encadré et entouré par les préparatifs et les négociations qui précèdent le jeu et par les négociations et les conflits que le jeu génère et qui invitent les joueurs à se redéfinir dans le contexte du jeu et en dehors du jeu (Bateson, 1956; Fein, 1979; Garvey et Berndt, 1977; Rubin, 1980; Schwatzman, 1976, 1978; Sutton-Smith, 1979).

\subsubsection{Les modalités spécifiques d'analyse des données de la deuxième question de recherche}

Pour opérationnaliser l'analyse de l'ensemble des composantes structurales, vues globalement dans l'activité de construction et dans l'activité de jeu symbolique, un tableau référence a été élaboré. Il permet de regrouper les éléments recherchés dans les épisodes observés. Chaque élément de la composante structurale est par la suite défini.

Ce tableau tient compte aussi de l'émergence de composantes non définies a priori. Ces composantes ou éléments permettront ainsi de déterminer des spécificités sur le plan symbolique dans le contexte de l'activité à partir du médium bloc (voir tableau 4.6). 
Tableau 4.6

Identification des éléments d'analyse retenus pour chacune des composantes structurales

\begin{tabular}{|c|c|c|c|}
\hline $\begin{array}{c}1^{\text {re }} \text { composante } \\
\text { Substitution } \\
\text { d'objets }\end{array}$ & $\begin{array}{l}\text { 2e }^{\text {composante }} \\
\text { Jeu social } \\
\text { ritualisé }\end{array}$ & $\begin{array}{c}3^{e} \text { composante } \\
\text { Adoption de rôles }\end{array}$ & $\begin{array}{l}\text { Composantes } \\
\text { émergentes }\end{array}$ \\
\hline $\begin{array}{l}\text { S.S.S. } \\
\text { Substitution } \\
\text { symbolique simple. } \\
\text { S.S.E.S. } \\
\text { Substitution } \\
\text { symbolique dans } \\
\text { l'élaboration d'un } \\
\text { scénario. } \\
\text { S.S.I.S. } \\
\text { Substitution } \\
\text { symbolique à } \\
\text { l'intérieur d'un } \\
\text { scénario. } \\
\end{array}$ & $\begin{array}{l}\text { C.S.I. } \\
\text { Comportement } \\
\text { symétrique } \\
\text { identique. } \\
\text { C.A.I. } \\
\text { Comportement } \\
\text { d'actions } \\
\text { intercoordonnées. } \\
\text { C.A.I.R. } \\
\text { Comportement } \\
\text { d'actions } \\
\text { intercoordonnées } \\
\text { renversées. }\end{array}$ & 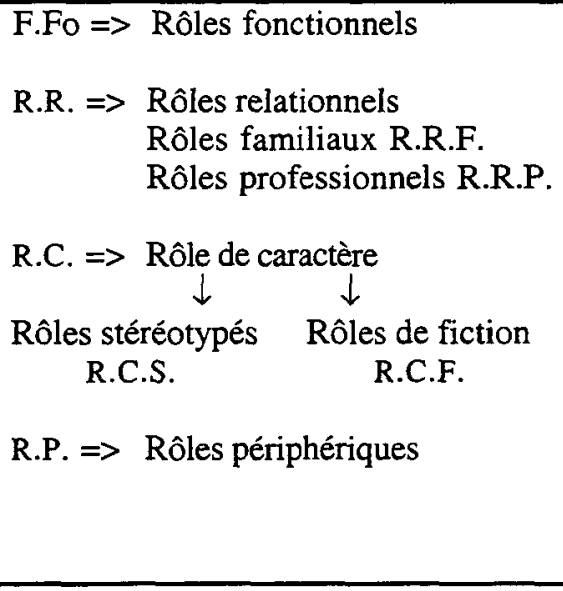 & \\
\hline & & $\begin{array}{c}4^{\mathrm{e}} \text { composante } \\
\text { Jeu fantaisiste thématique }\end{array}$ & \\
\hline & & R.Fa $=>$ Rôles fantaisistes & \\
\hline
\end{tabular}

- Analyse de la première composante structurale: la substitution
d'objets

Les substitutions symboliques d'objets sont analysées à partir des nombreux échanges issus de la situation de l'activité de construction et de l'activité de jeu symbolique. Les activités de transformations symboliques d'objets apparaissent habituellement dans le déroulement de l'observation, soit en séquences isolées, soit en scénario. Dans ce cas, elles peuvent se situer au début du jeu pour contribuer à la mise en place d'un thème; ou tout au cours du déroulement du thème pour soutenir son développement (Verba, 1990).

Trois types de substitutions symboliques d'objets sont retenus relativement aux données d'études antérieures: substitutions symboliques simples, substitutions symboliques dans l'élaboration d'un scénario et finalement, substitutions symboliques à l'intérieur du scénario.

\section{Substitution d'objets}

La substitution d'objets se définit comme étant l'habileté à assimiler un objet à un autre dans le jeu de faire-semblant. 
Substitutions symboliques "simples" (code d'analyse S.S.S.)

Il s'agit ici de séquences plus ou moins longues, constituées principalement par la dénomination des signifiés attribués aux objets (ex.: une courbe elliptique est appelée volant, un carrefour est appelé voiture). Dans ces séquences, il n'y a ni scénario, ni rôle, mais quelquefois de courtes actions de faire-semblant avec les objets transformés au plan symbolique. Plusieurs procédés sont à l'œuvre pour faire comprendre à l'autre les significations de ces objets: simple information, explication par répétition et par indication, intégration dans une description, précisions apportées spontanément ou par demande de clarification (Verba, 1990).

Substitutions symboliques dans l'élaboration d'un scénario (code d'analyse:

\section{S.S.E.S.)}

Substitution des objets en tant qu'éléments nécessaires au développement du thème. Les substitutions d'objets survenant au début des épisodes de jeu sont fréquentes et contribuent souvent à la mise en place du thème développé par les enfants.

Substitutions symboliques à l'intérieur du scénario (code d'analyse S.S.I.S.)

Les substitutions ponctuelles d'objets, faites au fur et à mesure du développement du thème: apparition en chapelet dans certains jeux; ces substitutions multiples et successives, opérées ad hoc, fixent et relient les divers éléments du scénario.

\section{- Analyse de la deuxième composante structurale: le jeu social ri- tualisé}

Dans cette étude, le jeu social ritualisé est analysé à partir des trois stades identifiés dans des études antérieures, quant aux divers comportements interactifs qui apparaissent dans le jeu symbolique, soit: les comportements symétriques identiques, les comportements d'actions intercoordonnées et finalement, les comportements d'actions intercoordonnées et renversées.

Le jeu social ritualisé concerne la relation du rôle entre les partenaires sociaux. Le faire-semblant ritualisé est gouverné par l'acceptation de ce qui apparaît être implicitement consenti sur le rôle de chacun des partenaires dans les séquences interactives. 


\section{Comportements symétriques identiques (code d'analyse C.S.I.)}

Le partenaire de jeu est probablement une copie exacte des effets physiques produits avec les jouets ou avec les objets par l'autre partenaire de jeu.

\section{Comportement d'actions intercoordonnées (code d'analyse C.A.I.)}

Les partenaires se conduisent dans des voies particulières ritualisées dans lesquelles les actions sont intercoordonnées. Chaque partenaire définit son rôle en fonction du rôle de l'autre, un médecin et un malade par exemple.

Comportements d'actions intercoordonnées renversées (code d'analyse C.A.I.R.)

L'intercoordination peut entraîner un renversement des rôles des partenaires, le malade devient le médecin et le médecin devient le malade. Cette intercoordination et cette réciprocité apparaissent au dernier stade des interactions sociales des rôles ritualisés.

\section{- Analyse de la troisième composante structurale: l'adoption de rôles}

L'adoption de rôles est analysée à partir des différents rôles qui sont susceptibles d'émerger dans l'activité de construction et dans l'activité de jeu symbolique: les rôles fonctionnels, les rôles relationnels, les rôles de caractère (stéréotypés et de fiction) et les rôles périphériques.

Le jeu de rôle implique la personnification d'un rôle, c'est-à-dire la simulation de l'identité qui permet de reconnaitre, de distinguer et d'apprivoiser les caractéristiques particulières et les tâches spécifiques d'un rôle donné. Il comporte la mise en scène avec une ou plusieurs personnes, d'un thème commun, où les objets et l'environnement sont transformés (Doyle, 1988; Doyle, Doehring et Delorimier, 1988; Fein, 1981).

- Rôles fonctionnels (code d'analyse R-Fo)

Rôles organisés par un objet ou une activité. Ils sont définis par l'action elle-même et sont distincts des rôles familiaux et de caractère.

- Rôles relationnels (code d'analyse R.R.)

Rôles familiaux (R.R.F.) qui impliquent des relations complémentaires (mère-enfants, mari-femme). 
Rôles professionnels (R.R.P.): cuisinier, mécanicien, etc.

- Rôles de caractère (code d'analyse R.C.)

Rôles stéréotypés (R.C.S.): pompier, policier, cow-boy, docteur.

Rôles de fiction (R.C.F.): issus d'histoires, de la télévision ou de la tradition orale. Personnages: Père Noël, Cendrillon, Batman.

- Rôles périphériques (code d'analyse R.P.)

Ce sont des rôles que les enfants initient mais dont ils n'assument jamais l'identité. C'est, en fait, un troisième personnage réel ou imaginaire qui n'entre jamais dans l'espace de jeu proprement dit.

- Analyse de la quatrième composante structurale: le jeu fantaisiste thématique/le jeu sociodramatique

Cette quatrième composante structurale concernant le jeu fantaisiste thématique est analysée par l'élément rôle fantaisiste qui vient compléter la liste des différents rôles identifiés dans la composante structurale: adoption de rôles. Il s'agit, lors de l'analyse, d'être sensible d'abord, à la présence du rôle fantaisiste et, par la suite, d'en analyser le contexte de son élaboration.

Rôles fantaisistes (code d'analyse R.Fa)

C'est un rôle basé sur des thèmes fantaisistes inspirés d'un monde imaginaire impliquant des personnages peu communs mais différents des rôles de caractère de fiction (ex.: sorcier, extraterrestre).

- Analyse de la cinquième composante structurale: la structuration du jeu

L'analyse de la structuration du jeu fait ici référence aux actes de communication qui assurent différentes fonctions dans l'élaboration du jeu. Ces actes de communication transmettent, d'une part, des informations sur le contenu du jeu et de son déroulement et, d'autre part, des informations sur la nature de la relation interpersonnelle des partenaires de jeu. 
Puisque dans cette étude la communication est abordée sous l'angle des interactions sociales, nous analyserons donc la structuration du jeu à l'intérieur même des éléments d'analyse liés à la troisième question de recherche.

\subsubsection{La mise en forme des données}

Pour cette deuxième question de recherche, sont analysées globalement les diverses composantes structurales de l'activité de construction et de l'activité de jeu symbolique.

La mise en forme des données concernant les composantes structurales du jeu symbolique s'est réalisée par un premier regroupement selon les diverses formes de participation sociale. Le choix de cet élément de regroupement permet de mettre en évidence la structure d'organisation de l'activité de construction et de l'activité de jeu symbolique dans un mode de participation solitaire ou parallèle, associatif et coopératif. Le tableau suivant présente la structure d'organisation de ces deux activités. 
Tableau 4.7

Structure d'organisation de l'activité de construction et de jeu symbolique selon les différentes formes de participation sociale et le temps accordé pour chaque type d'activités

\begin{tabular}{|c|c|c|c|c|c|c|c|c|}
\hline \multirow{2}{*}{$\begin{array}{c}\text { Forme de } \\
\text { participation sociale }\end{array}$} & \multirow{2}{*}{\multicolumn{2}{|c|}{$\begin{array}{c}\text { Jeu solitaire/ } \\
\text { parallèle }\end{array}$}} & \multicolumn{6}{|c|}{ Jeu associatif, coopératif } \\
\hline & & & \multicolumn{2}{|c|}{2} & \multicolumn{2}{|c|}{3} & \multicolumn{2}{|c|}{$\overline{4}$} \\
\hline Séances/temps en minutes & A.C. & A.J.S. & A.C. & A.J.S. & A.C. & A.J.S. & A.C. & A.J.S. \\
\hline Séance 1 & $\begin{array}{l}13 \\
7 \\
9\end{array}$ & $\begin{array}{c}- \\
7 \\
12 \\
5\end{array}$ & & & & & & \\
\hline Séance 2 & & & $\begin{array}{l}32 \\
32\end{array}$ & $\begin{array}{l}7 \\
9\end{array}$ & & & & \\
\hline & & & $\begin{array}{l}32 \\
32\end{array}$ & $\begin{array}{l}12 \\
12\end{array}$ & & & & \\
\hline \multirow[t]{2}{*}{ Séance 3} & $\begin{array}{l}18 \\
42\end{array}$ & 24 & & & & & & \\
\hline & & & $\begin{array}{l}* 34 \\
* 34\end{array}$ & $\begin{array}{l}8 \text { min. } \\
\text { à l'int. } \\
\text { de l'act } \\
\text { de } \\
\text { const. }\end{array}$ & & & & \\
\hline \multirow[t]{2}{*}{ Séance 4} & $\begin{array}{l}17 \\
33\end{array}$ & $\begin{array}{l}26 \\
10\end{array}$ & & & & & & \\
\hline & & & $\begin{array}{l}20 \\
20\end{array}$ & $\begin{array}{l}23 \\
23\end{array}$ & & & & \\
\hline Séance 5 & & & & & & & $\begin{array}{l}30 \\
30 \\
30 \\
30 \\
\end{array}$ & $\begin{array}{c}- \\
\frac{19}{7}\end{array}$ \\
\hline \multirow[t]{3}{*}{ Séance 6} & 21 & 27 & & & & & & \\
\hline & & & $\begin{array}{c}31 \\
5\end{array}$ & $\begin{array}{l}17 \\
43 \\
\end{array}$ & & & & \\
\hline & 25 & 16 & & & & & & \\
\hline \multirow[t]{2}{*}{ Séance 7} & & & $\begin{array}{l}22 \\
22\end{array}$ & $\begin{array}{l}7 \\
8\end{array}$ & & & & \\
\hline & & & $\begin{array}{l}11 \\
11\end{array}$ & $\begin{array}{l}* 25 \\
* 25 \\
\end{array}$ & & & & \\
\hline \multirow[t]{2}{*}{ Séance 8} & & & $\begin{array}{l}11 \\
11\end{array}$ & $\begin{array}{l}36 \\
36\end{array}$ & & & & \\
\hline & & & $\begin{array}{l}36 \\
36\end{array}$ & - & & & & \\
\hline \multirow[t]{3}{*}{ Séance 9} & & & 22 & 20 & & & & \\
\hline & & & $\begin{array}{l}* 22 \\
* 22\end{array}$ & $\begin{array}{l}22 \\
22\end{array}$ & & & & \\
\hline & & & 15 & $\overline{28}$ & & & & \\
\hline
\end{tabular}

* L'astérisque indique les séances où il y a simultanéité de l'activité de construction et de l'activité de jeu symbolique. 


\section{- L'organisation solitaire}

Les séances 1, 3, 4 et 6 présentent dix organisations solitaires de l'activité de construction et de l'activité de jeu symbolique des enfants réalisant individuellement des projets globaux et secondaires. Il s'agit d'examiner la présence et l'organisation de certains éléments de jeu symbolique dans cette forme de participation sociale.

\section{- $\quad$ Les substitutions d'objets}

Pour chaque projet individuel, on dénote que l'activité de construction est l'activité de base pour toutes les séances (sauf SEA1-ENF3). Même dans certains cas, si le temps consacré à l'activité de jeu symbolique est supérieur au temps consacré à l'activité de construction, celle-ci demeure l'activité de base sur laquelle s'élabore une activité de jeu symbolique.

Dans l'ensemble des dix organisations solitaires, le projet global de construction a engendré quelques substitutions symboliques simples d'objets. Elles sont des éléments qui viennent relancer un possible de jeu symbolique mais demeurent des éléments initiatiques annoncés sans toutefois donner naissance à l'élaboration de scénarios. Elles sont annoncées dans certains cas et exécutées dans le contexte de l'activité de construction pour alimenter et soutenir l'élaboration de cette activité.

SEA3-ENF2
S.S.S.1 20:06:03
S.S.S. $2 \quad 33: 24: 20$
S.S.S.3 $35: 45: 17$
Ça c'est une plage.
Deux demi-cercles pour les deux portières de sa voiture.
Prend un demi-cercle et imite le geste du volant.

SEA4-ENF2: Dans un projet global annoncé comme .

S.S.S.1 06:05:03 Prénom, regarde mon envoyeur de fumée (double module).

S.S.S.2 11:48:17 Fait le geste d'un volant (colonne double).

S.S.S.3 11:55:21 Aïe, y'a pas de dynamites dans mon bateau.

$\mathrm{Ah}$, je vas m'en prendre (modules).

Certaines substitutions simples d'objets sont retenues parce qu'elles ont été entendues d'un autre membre de l'aire des blocs et reprises par l'enfant pour son compte, sans toutefois constituer une trame personnelle de sa propre activité de construction.

SEA4-ENF2: Dans un projet global annoncé comme "une auto".

S.S.S.4 22:23:13 Tiens mon fusil est installé! 
On dénote également des substitutions symboliques simples d'objets très isolées du contexte du projet global, c'est-à-dire sans lien avec le projet global, comme si une substitution simple d'objets se réalisait mais en ne s'inscrivant pas de manière significative dans l'activité de construction de l'enfant.

SEA6-ENF4: Dans un projet global annoncé comme "un royaume".

S.S.S.1 04:09:02 Mitraillette (double module)

- L'adoption des rôles

Dans cette forme individuelle et parallèle de réalisation des projets de construction, une autre composante structurale apparaît, soit celle de l'adoption de rôles. Les rôles éventuels amorcés le sont par le biais de paroles paralinguistiques ou d'onomatopées qui sont:

- en lien avec la manipulation spontanée d'un accessoire de jeu tel les voitures:

SEA1-ENF2

07:30:00 Il fait rouler sa voiture sur la planche inclinée en imitant le vrombissement de la voiture "VRVRVRVRVRVRVR"

10:41:21 Il imite le son de la sirène en déplaçant une voiture.

11:05:00 Le pompier arrive en faisant rouler la voiture.

12:00:00 Il imite le son de la sirène.

- en lien avec le projet global de l'activité de construction:

SEA3-ENF1: Dans un projet global annoncé comme "le château des fantômes".

07:58:12 Elle prend un personnage de bois et on entend (et continue à construire).

18:26:22 Elle prend un personnage de bois (rouge), le déplace dans l'espace en imitant le son du fantôme. Finalement, elle le place au-dessus de la tour. Elle place les personnages à différents endroits en imitant le "Hou! Hou! Hou!" du fantôme.

ou encore, reconnus par l'observateur, mais non explicites par l'enfant, parce qu'ils impliquent des gestes imitatifs d'un rôle social donné comme celui du conducteur d'une voiture:

SEA3-ENF2: Dans un projet global non explicite annoncé comme "une auto".

33:24:20 Elle rentre et s'assoit dans sa construction (les deux demi-cercles semblent servir de porte, parce qu'elle les a placés de chaque côté).

35:45:17 Elle fait un volant avec son demi-cercle. Elle imite les gestes du conducteur. 
Finalement, dans l'ensemble de ces projets individuels, se dégage la présence d'un ou deux épisodes de l'activité de jeu symbolique de très courte durée découlant prioritairement de l'activité de construction:

SEA3-ENF1: Dans un projet global annoncé comme "le château des fantômes".

Épisode 1: Des fantômes.

Elle déplace des fantômes dans l'espace.

Elle place des fantômes sur différents cylindres (tours) du château.

Elle entretient un dialogue entre les personnages fantômes.

Elle chante fantômie, des fantômes.

SEA4-ENF2: 2 épisodes: Projet global = bateau

Épisode 1: Explosion de dynamites.

20:07:02 à 20:43:23 Je veux pas le faire sauter moi, à cause j'ai plein de toutes sortes de dynamites dedans. Aïe! Attention, hein! Y'a encore plein de fusils.

Épisode 2: Pente pour faire rouler les voitures

34:26:10 à 36:52:19 (Prénom) Aïe (Prénom)! Si tu veux venir te glisser dans ma côte-là avec une auto, tu peux, regarde, y'a deux côtés pour toutes les autres. Veux-tu venir? À part ça, à va loin ma côte-là, regarde griooooooo (en suivant le parcours de la pente). À va jusqu'en bas.

SEA6-ENF1: Un épisode: Projet global: cage à voleurs.

Épisode 1: 20:35:14 à 26:15:00

20:35:14 Tu te demandes par où je vas rentrer?

21:02:28 Elle prend des personnages de bois (5), déplace les deux blocs horizontaux et fait un espace pour introduire les personnages de bois.

23:34:23 Fermer, tous les voleurs sont là-dedans (dans son enclos).

\section{- L'organisation dyadique}

Les séances $2,3,4,6,7,8$ et 9 présentent 11 organisations dyadiques de l'activité de construction et de l'activité de jeu symbolique des enfants réalisant des projets globaux et complémentaires. Voici donc comment se constitue la présence des éléments de jeu symbolique dans cette forme de participation sociale qu'est la dyade.

Pour chacune des 11 organisations dyadiques, la présence des composantes structurales se répartit dans l'activité de construction et dans l'activité de jeu symbolique.

De ces 11 organisations dyadiques, dix présentent une activité de jeu symbolique subordonnée à l'activité de construction. Les enfants élaborent des projets globaux et secondaires dans l'activité de construction et, par la suite, ces projets donnent lieu à l'élaboration de scénarios dans l'activité de jeu symbolique. 
De ces dix organisations dyadiques, trois présentent une activité de jeu symbolique simultanée à l'activité de construction. Dans ces organisations, l'activité de construction constitue toujours l'activité de base; cependant, l'apparition de l'activité de jeu symbolique se réalise sous différentes formes d'agencement.

- La simultanéité épisodique de courte durée de l'activité de construction dans l'activité de jeu symbolique.

Une des formes d'agencement observée est l'activité de jeu symbolique subordonnée à l'activité de construction. L'élaboration de scénarios se réalise à partir du contexte créé et, par la suite, l'activité de jeu symbolique s'organise simultanément à l'activité de construction. Donc, des éléments de construction génèrent une activité de jeu symbolique et à son tour, l'activité de jeu symbolique génère des projets secondaires de construction. Il y a simultanéité des deux activités dans la dominance de l'activité de jeu symbolique.

\begin{tabular}{|c|c|c|}
\hline SEA7-ENF3-4 & Activité de construction & Activité de jeu symbolique \\
\hline Durée totale: 35 minutes & 11 premières minutes & $\begin{array}{c}\text { Simultanéité épisodique de courte } \\
\text { durée de l'activité de construction } \\
25 \text { minutes }\end{array}$ \\
\hline
\end{tabular}

La SEA7-ENF3-4 illustre cette forme d'agencement. Dans cette séance, les 11 premières minutes sont consacrées exclusivement à l'activité de construction. Par la suite, l'activité de jeu symbolique se greffe à l'activité de construction et s'organise en simultanéité impliquant une activité de construction épisodique plus ou moins élaborée.

- La simultanéité épisodique de courte durée de l'activité de jeu symbolique dans l'activité de construction.

Une autre forme d'agencement observée est la simultanéité épisodique de courte durée de l'activité de jeu symbolique à l'intérieur de l'activité de construction. L'activité de jeu symbolique apparait épisodiquement dans l'activité de construction.

Dans la SEA3-ENF3-4, les cinq premières minutes sont essentiellement consacrées à l'activité de construction orientée à élaborer un projet global. Par la suite, l'activité de construction se poursuit encore pendant 29 minutes, mais plusieurs épisodes d'activité de jeu symbolique apparaissent simultanément à l'activité de construction et les deux parte- 
naires de jeu partagent les significations de ces épisodes de courte durée, qui s'intercalent à différents moments dans l'activité de construction. Par la suite, les huit dernières minutes sont essentiellement consacrées à l'activité de jeu symbolique.

\begin{tabular}{|c|c|c|}
\hline $\begin{array}{c}\text { SEA3-ENF3-4 } \\
\text { ACTIVITÉ DE CONSTRUCTION }\end{array}$ & $\begin{array}{c}\text { Activité de construction } \\
\text { Simultanéité épisodique } \\
\text { de courte durée de l'activité } \\
\text { de jeu symbolique }\end{array}$ & $\begin{array}{l}\text { Activité de jeu } \\
\text { de construction }\end{array}$ \\
\hline 5 premières minutes & 29 minutes & 8 dernières minutes \\
\hline & 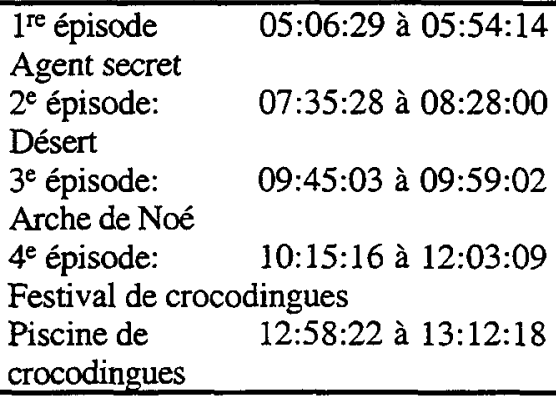 & \\
\hline
\end{tabular}

- La simultanéité épisodique de longue durée de l'activité de jeu symbolique dans l'activité de construction.

Une troisième forme d'agencement observée est la simultanéité épisodique de longue durée à l'intérieur de l'activité de construction.

\begin{tabular}{|c|c|c|c|}
\hline SEA9-ENF2-3 ACTIVITE DE CONSTRUCTION & \\
\hline $\begin{array}{c}\text { ACTIVITÉ } \\
\text { DE CONSTRUCTION }\end{array}$ & $\begin{array}{c}\text { Activité de construction } \\
\text { Simultanéité épisodique } \\
\text { de longue durée } \\
\text { de l'activité } \\
\text { de jeu symbolique }\end{array}$ & Activité de construction & $\begin{array}{c}\text { Activité } \\
\text { de jeu symbolique }\end{array}$ \\
\hline 15 premières minutes & $15: 13: 14$ à $21: 15: 29$ & 1 minute & 22 dernières minutes \\
\hline \multicolumn{2}{|c|}{22 minutes } & \multicolumn{1}{|c|}{} \\
\hline
\end{tabular}

Les 22 premières minutes de la SEA9-ENF3 sont consacrées à l'activité de construction. Cependant, une trame de six minutes d'activité de jeu symbolique s'infiltre dans l'activité de construction. Le thème de cette trame de jeu symbolique origine du projet global du bateau, et la proposition de la destruction du bateau à l'aide d'un canon constitue cette trame de six minutes. Par la suite, les enfants continuent leur activité de construction et une activité de jeu symbolique s'élabore au cours des 22 dernières minutes. 
Donc, l'ensemble des trois projets dyadiques simultanés ont impliqué deux types de simultanéité. Une simultanéité d'activité de jeu symbolique à l'intérieur d'une activité de construction qui se réalise comme suit: une activité de base qui est une activité de construction. Les enfants élaborent des projets globaux et secondaires et ceux-ci engendrent une simultanéité épisodique d'activité de jeu symbolique plus ou moins élaborée, impliquant un ensemble d'éléments constituant le jeu symbolique. Par la suite, après l'élaboration d'une trame plus ou moins longue de scénarios, les enfants continuent l'activité de construction. Finalement, ils amorcent une activité de jeu symbolique subordonnée à l'activité de construction.

Le deuxième type de simultanéité s'observe lorsqu'une activité de construction se retrouve à l'intérieur d'une activité de jeu symbolique. Au départ, l'activité de base est toujours une activité de construction et, par la suite, l'activité de jeu symbolique se subordonne à celle-ci. Les enfants initient et élaborent des scénarios et ceux-ci engendrent une simultanéité épisodique d'activité de construction plus ou moins élaborée pour supporter et alimenter le jeu symbolique. Par la suite, les enfants continuent leur trame de jeu symbolique.

Dans chacune de ces organisations dyadiques, l'agencement des composantes structurales se définit en fonction de l'activité de jeu symbolique subordonnée ou simultanée à l'activité de construction. Ainsi, les premiers questionnements intuitifs de cette problématique de recherche supposaient une activité de jeu symbolique subordonnée ou simultanée à l'activité de construction. Cependant, l'analyse de l'organisation structurelle de l'activité de l'enfant au coin bloc révèle que l'activité de construction constitue, dans la majorité des organisations solitaires, dyadiques ou à quatre participants, l'élément moteur de l'activité de l'enfant. Par ailleurs, l'activité de jeu symbolique peut être simultanée épisodique de courte ou de longue durée dans l'activité de construction et elle peut être subordonnée à cette activité. Par contre, l'activité de construction peut également devenir simultanée épisodique de courte ou de longue durée à l'activité de jeu symbolique subordonnée.

Une fois les diverses formes d'agencement de l'organisation dyadique présentées, nous nous attardons aux différentes composantes structurales. 
- Les substitutions d'objets et l'activité de jeu symbolique subordonnée à l'activité de construction.

Les séances $2,4,6,7,8$ et 9 représentent une organisation ludique dyadique où l'on observe une activité de jeu symbolique subordonnée à l'activité de construction. Les enfants élaborent, en premier lieu, des projets globaux et secondaires et, par la suite, initient une activité de jeu symbolique à partir des différents projets réalisés. Les substitutions symboliques simples d'objets s'effectuent dans le contexte de l'activité de construction et les autres substitutions dans le contexte de l'activité de jeu symbolique.

Dans ces différentes séances, la première composante structurale, soit la substitution d'objets, est d'abord observée dans l'activité de construction. En effet, l'activité de construction, elle-même, génère un certain nombre de substitutions symboliques d'objets répondant à différentes fonctions.

L'observation de l'activité de construction a permis de constater l'existence de 26 substitutions symboliques simples d'objets et dans l'activité de jeu symbolique, quatre substitutions symboliques dans l'élaboration d'un scénario et 28 substitutions symboliques à l'intérieur d'un scénario. Ces différentes substitutions sont présentées au tableau 4.8 .

Tableau 4.8

Les différentes substitutions d'objets des séances dyadiques de l'activité de jeu symbolique subordonnée à l'activité de construction

\begin{tabular}{|l|c|c|c|}
\hline \multicolumn{1}{|c|}{ SEAANCE } & $\begin{array}{c}\text { Substitutions } \\
\text { symboliques simples } \\
\text { d'objets }\end{array}$ & $\begin{array}{c}\text { Substitutions } \\
\text { symboliques } \\
\text { dans l'élaboration } \\
\text { d'un scénario }\end{array}$ & $\begin{array}{c}\text { Substitutions } \\
\text { symboliques d'objets } \\
\text { à l'intérieur } \\
\text { d'un scénario }\end{array}$ \\
\hline SEA2-ENF1-2 & 1 & 1 & 3 \\
\hline SEA2-ENF3-4 & 3 & 3 & 1 \\
\hline SEA4-ENF3-4 & 5 & & 7 \\
\hline SEA6-ENF2-3 & 2 & & 2 \\
\hline SEA7-ENF1-2 & 1 & & \\
\hline SEA8-ENF1-2 & & & 15 \\
\hline SEA8-ENF3-4 & 6 & & 28 \\
\hline SEA9-ENF1-4 & 8 & 4 & \\
\hline TOTAL & 26 & & \\
\hline
\end{tabular}


La première fonction, accordée par les enfants, est l'attribution d'une signification particulière à un bloc comme élément s'intégrant et facilitant la représentation matérielle d'un projet de construction en cours d'élaboration. Ainsi, pour répondre à des besoins immédiats de projet de construction, les enfants attribuent une signification particulière à un bloc de bois et partagent cette nouvelle signification avec le partenaire de jeu. Cette nouvelle signification s'inscrit dans l'ajout de projets complémentaires du projet global ou d'un projet secondaire initié.

Les exemples suivants illustrent cette première fonction des substitutions symboliques simples d'objets dans l'activité de construction.

\begin{tabular}{|llllll|}
\hline & Projet global & \multicolumn{2}{c|}{$\begin{array}{l}\text { Substitutions symboliques } \\
\text { simples d'objets }\end{array}$} & Bloc utilisé \\
SEA2-ENF3-4 & Maison & $07: 21: 13$ & Barrière & \\
SEA4-ENF3-4 & Bateau & $04: 29: 27$ & Moteur & Quadrangle \\
& & $07: 25: 05$ & Envoyeur de fumée & Planche de toit \\
& & $11: 55: 06$ & Dynamites & Mourbes elliptiques \\
& & $16: 06: 08$ & Muffler & Modules \\
SEA6-ENF2-3 & Autoroute & $10: 22: 29$ & Pont & Bloc en Y \\
SEA8-ENF3-4 & Maison & $10: 35: 26$ & Eau froide, chaude & Cylindres \\
& & $14: 07: 03$ & Oiseau & Demi-carrefour \\
& & $14: 40: 03$ & Eau froide, eau chaude & 2 triangles \\
& & $17: 48: 06$ & Téléviseur & 2 carrefours \\
SEA9-ENF1-4 & Château & $00: 57: 14$ & Porte & Bloc en H \\
& Bateau & $02: 07: 03$ & Bombe & Modules \\
& & $03: 07: 13$ & Porte & Bloc en Y \\
& & $06: 27: 03$ & Bombe & Colonnes \\
& & $17: 42: 18$ & Fusil & \\
\hline
\end{tabular}

La deuxième fonction est l'attribution d'une signification particulière à un agencement de blocs pour déterminer un espace spécifique dans le projet de construction en cours d'élaboration, telle que présentée dans les exemples qui suivent.

\begin{tabular}{|lllll|}
\hline & Projet global & \multicolumn{2}{l|}{$\begin{array}{l}\text { Substitutions symboliques } \\
\text { simples d'objets }\end{array}$} & Bloc utilisé \\
SEA2-ENF3-4 & Maison & $08: 44: 07$ & Balcon & \\
& & $08: 48: 28$ & Porte & Arches \\
SEA7-ENF1-2 & Château & $03: 58: 05$ & Passerelle & . \\
\hline
\end{tabular}


La troisième fonction est l'attribution d'une signification particulière d'un bloc comme personnage-animal comme l'illustre l'exemple suivant.

\begin{tabular}{|lllll|}
\hline & Projet global & \multicolumn{2}{l}{$\begin{array}{l}\text { Substitutions symboliques } \\
\text { simples d'objets }\end{array}$} & Bloc utilisé \\
SEA9-ENF2-3 & Bateau & $25: 06: 05$ & Crocodile & Carrefour \\
\hline
\end{tabular}

Également, certaines substitutions simples d'objets sont réalisées non pas à partir du matériel de base, les blocs, mais à partir du matériel accessoire. L'exemple qui suit est éloquent.

\begin{tabular}{|llll|}
\hline & $\begin{array}{l}\text { Projet } \\
\text { global }\end{array}$ & $\begin{array}{l}\text { Substitutions symboliques } \\
\text { simples d'objets }\end{array}$ & $\begin{array}{l}\text { Matériel accessoire } \\
\text { utilisé }\end{array}$ \\
SEA9-ENF1-4 & $09: 36: 16$ & $\begin{array}{l}\text { Manette } \\
\text { Boutons des manettes }\end{array}$ & $\begin{array}{l}\text { Panneau de signalisation } \\
\text { Embout de caoutchouc des } \\
\text { panneaux de signalisation } \\
\text { adhérant à une surface lisse }\end{array}$ \\
SEA3-ENF3-4 & Vo:57:08 & Voitures miniatures \\
\hline
\end{tabular}

Finalement, une seule des substitutions relevées s'est réalisée de manière isolée sans liens avec le projet global de construction.

\begin{tabular}{|llll|}
\hline & $\begin{array}{l}\text { Projet } \\
\text { global }\end{array}$ & $\begin{array}{l}\text { Substitutions symboliques } \\
\text { simples d'objets } \\
\text { SI9:07:22 }\end{array}$ & Matériel utilisé \\
SEA3-ENF3-4 & & Palles & \\
\hline
\end{tabular}

À la lumière de ces données, il est possible de constater que l'ensemble des substitutions symboliques simples d'objets dans les dyades sont produites dans l'activité de construction pour répondre à trois fonctions spécifiques. La plus usuelle de ces fonctions est l'attribution d'une signification particulière à un bloc comme élément s'intégrant et facilitant la représentation matérielle du projet de construction en cours d'élaboration. Cependant, les deux autres fonctions ne sont pas négligeables même si elles apparaissent plus rarement. Elles indiquent d'autres modes de substitutions symboliques d'objets possibles à exploiter par les enfants. En outre, elles traduisent des moyens significatifs différents afin de résoudre des problèmes rencontrés dans l'activité de construction. 
- Les substitutions d'objets et l'activité de jeu symbolique simultanée à l'activité de construction.

Les substitutions symboliques simples d'objets dans les dyades où l'activité de jeu symbolique est simultanée à l'activité de construction se regroupent dans les séances 3,7 et 9. Le tableau 4.9 présente la répartition de ces substitutions.

Tableau 4.9

La répartition des substitutions symboliques d'objets dans les dyades où l'activité symbolique est simultanée à l'activité de construction

\begin{tabular}{|l|c|c|c|}
\hline SÉANCES & $\begin{array}{c}\text { Substitutions symbo- } \\
\text { liques simples d'objets }\end{array}$ & $\begin{array}{c}\text { Substitutions symbo- } \\
\text { liques } \\
\text { à l'élaboration } \\
\text { d'un scénario }\end{array}$ & $\begin{array}{c}\text { Substitutions symbo- } \\
\text { liques d'objets } \\
\text { à l'intérieur } \\
\text { d'un scénario }\end{array}$ \\
\hline SEA3-ENF3-4 & 8 & 0 & 16 \\
\hline SEA7-ENF3-4 & 4 & 1 & 6 \\
\hline SEA9-ENF2-3 & 2 & 0 & 5 \\
\hline TOTAL & 14 & 1 & 27 \\
\hline
\end{tabular}

Ces séances totalisent 14 substitutions symboliques simples d'objets et 27 substitutions symboliques à l'intérieur d'un scénario. Dans ces séances, les substitutions symboliques simples d'objets se retrouvent pour la plupart, dans l'activité de construction et ne soutiennent pas les épisodes de l'activité de jeu symbolique simultanée à l'activité de construction. Elles sont produites pour répondre aux besoins dans l'activité de construction et se situent en dehors des épisodes de courte ou de longue durée d'activité de jeu symbolique dans l'activité de construction. Les exemples suivants illustrent quelques substitutions symboliques simples d'objets dans l'activité de construction.

\begin{tabular}{|llllll|}
\hline Séances & Projet global & \multicolumn{2}{l|}{$\begin{array}{l}\text { Substitutions symboliques } \\
\text { simples }\end{array}$} & Bloc utilisé \\
SEA4-ENF3-4 & Château & $01: 39: 15$ & Brique & \\
& & $06: 25: 24$ & Piste & Modules \\
& & $08: 47: 19$ & Marche & Demi-lune \\
& & $09: 02: 00$ & Tour & Petit cylindre \\
& & $09: 39: 20$ & Fauteuil & \\
SEA7-ENF3-4 & Poste de police & $01: 55: 02$ & Bonhommes de police & Carrefour \\
& & $02: 04: 05$ & Fenêtre & Voitures miniatures \\
& & $03: 02: 00$ & Autos de police & \\
\hline
\end{tabular}




\begin{tabular}{|lllll|}
\hline Séances & Projet global & $\begin{array}{l}\text { Substitutions symboliques } \\
\text { simples d'objets }\end{array}$ & Bloc utilisé \\
SEA3-ENF3-4 & $\begin{array}{l}\text { Maison } \\
\text { Projets secondaires } \\
\text { cabine de toilette } \\
\text { salon }\end{array}$ & $21: 23: 20$ & Cheminée & \\
& $\begin{array}{l}\text { cuisine } \\
\text { Maison-bateau }\end{array}$ & $26: 18: 21$ & Lumière & \\
& Bateau & $25: 06: 06$ & Crocodiles & $\begin{array}{l}\text { grandes planches } \\
\text { de toit }\end{array}$ \\
SEA9-ENF2-3 & & & & Carrefours \\
\hline
\end{tabular}

Par contre, certaines substitutions symboliques d'objets sont produites dans l'activité de jeu symbolique simultanée à l'activité de construction et à ce moment, elles ont comme fonction d'alimenter et de soutenir l'activité de jeu symbolique et l'activité de construction. Les substitutions symboliques simples servent donc le scénario amorcé comme les exemples suivants le montrent.

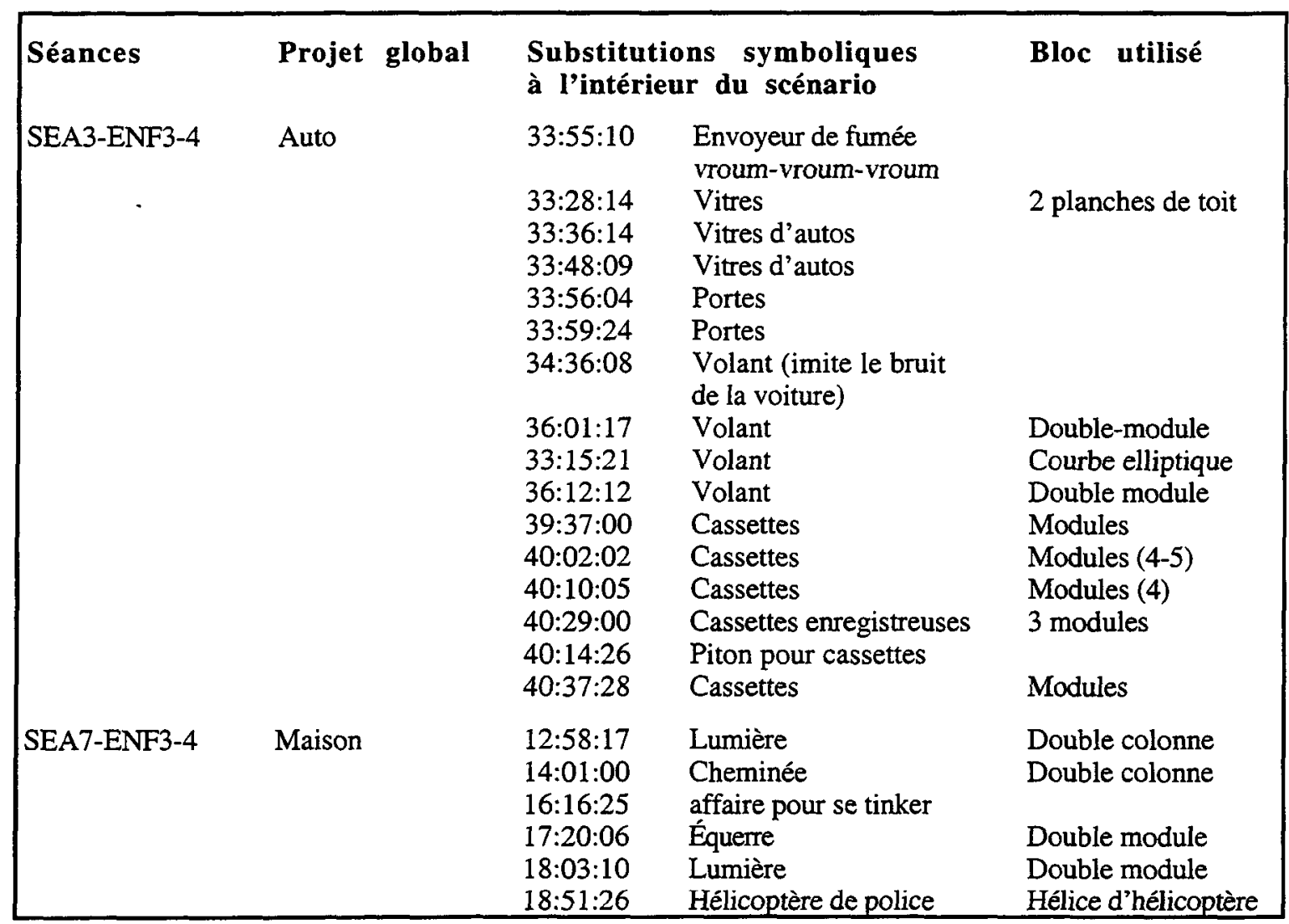




\begin{tabular}{|c|c|c|c|c|}
\hline Séances & Projet global & \multicolumn{2}{|c|}{$\begin{array}{l}\text { Substitutions symboliques } \\
\text { à l'intérieur du scénario }\end{array}$} & Bloc utilisé \\
\hline SEA9-ENF2-3 & Bateau & $\begin{array}{l}16: 53: 00 \\
17: 31: 19 \\
35: 29: 03 \\
35: 35: 17 \\
35: 42: 27 \\
36: 25: 20 \\
39: 53: 10 \\
40: 17: 19 \\
41: 19: 25 \\
41: 26: 08 \\
41: 55: 20\end{array}$ & $\begin{array}{l}\text { Poudre de canon } \\
\text { Bateau de canon } \\
\text { Pétards } \\
\text { Fusil } \\
\text { Bazookas } \\
\text { Bombe } \\
\text { Bateaux (3) } \\
\text { Plus grosse bombe } \\
\text { Bombe } \\
\text { Bateaux (4) } \\
\text { Bombe }\end{array}$ & $\begin{array}{l}\text { Gros cylindre } \\
\text { Quadrangle } \\
\text { Quadrangles } \\
\text { Quadrangle } \\
\text { Quadrangle } \\
\text { Quadrangles (3) } \\
\text { Quadrangle } \\
\text { Quadrangle } \\
\text { Quadrangles (4) } \\
\text { Module }\end{array}$ \\
\hline
\end{tabular}

Ces substitutions symboliques d'objets à l'intérieur d'un scénario rejoignent la définition de Verba (1990).

Des substitutions ponctuelles d'objets, faites au fur et à mesure du développement du thème, l'apparition en "chapelet" dans certains jeux. Les substitutions multiples et successives, opérées ad hoc fixent et relient les divers éléments du scénario. (Verba, 1990, p. 43)

\section{- L'organisation dyadique et l'adoption de rôles}

Dans les organisations dyadiques, les enfants nomment, initient et jouent différents rôles. Il serait peu opportun d'énumérer les différents rôles indépendant du contexte car la teneur de ces rôles prend sa signification même dans le contexte où ils sont nommés, initiés et joués. C'est pourquoi nous examinerons les rôles à l'intérieur des séquences ou des épisodes où ils prennent naissance, car ce n'est pas tant l'identification du rôle qui est important mais plutôt ce qu'il engendre dans l'activité de l'enfant.

\section{- L'adoption de rôles et l'activité de construction}

L'adoption de rôles est envisagée d'emblée dans l'activité de jeu symbolique, c'està-dire dans une trame impliquant tout un ensemble d'éléments constituant le jeu symbolique. Cependant, dans cette étude, on relève plusieurs épisodes où l'adoption de rôles est utilisée également dans l'activité de construction. Un premier rôle qui semble non significatif mais qui apparaît dans la SEA2-ENF1-2 à 03:02:21, le rôle de bébé. "Nous autres on a un petit bébé d'un an". Cet énoncé survient à la suite d'une altercation entre deux enfants et défend l'idée qu'il n'est pas un "bébé la la" quand un autre enfant reprend 
l'idée de bébé et la transpose dans une fiction et finalement, tout s'estompe. Donc, un rôle périphérique est nommé celui du bébé d'un an, mais n'est jamais joué. Par contre, il est fonctionnel car il est issu de l'action même de ce qui se passe, sans intention préalablement établie, sans cadre pouvant se réaliser et sans but apparent dans la poursuite du jeu.

Il est donc nommé et suscité par un élément de la situation qui se produit, ici et maintenant, et il est abandonné aussitôt nommé, mais il a pour fonction de neutraliser l'action en cours (altercation) et permet la poursuite de ce qui a été commencé sans changer le cours de cette activité. Il peut sembler être, pour l'observateur extérieur ponctuel, un élément isolé dans l'action qui s'élabore mais il est lié à un élément déclencheur dans la situation.

La SEA7-ENF3-4 présente deux séquences dans l'activité de construction où des rôles sont nommés.

PREMIÈRE SÉOUENCE:

01:01:16 Projet global annoncé: poste de police.

01:17:08 Ok. Ok. Que la porte à serait fermée (quatre demi-modules et une planche de toit, porte en $Y$ ).

01:30:03 Aïe (prénom de l'enfant, trois fois)! Tu sais les polices y passent sa rouge, les police, pis les pompiers aussi y passent sa rouge, pis les remorques aussi eux autres

01:53:03 qui ont des "wawers", y passent sa rouge aussi.

01:55:02 Ben oui, c'tun bonhomme de police (bloc carrefour).

[...] Toutes les pompiers à moi je l'en prends. Toi tu prends toutes les polices, moi je prends toutes les pompiers.

02:56:04 Bon aussi j'ai besoin des pompiers! Ous'qui sont hein? Ça t'a toé.

Dans cette séquence, les rôles nommés s'inscrivent en lien avec le projet global de construction, le poste de police. Ils sont nommés, pas joués. Ils sont donc, pour cette séquence, des rôles qualifiés de fonctionnels et de périphériques. Par contre, ces rôles fonctionnels ont pour but de traduire certaines connaissances de l'enfant relatives au thème qui est en cours d'élaboration dans l'activité de construction, soit le poste de police. Les rôles nommés de pompiers, de polices, de bonhomme de police, permettent "d'asseoir" les connaissances de chacun en regard du projet global qui se construit et 
permettent, également, aux enfants de partager des connaissances selon l'acceptation de chacune de ces significations pour la poursuite de l'activité. Dans cette séquence, et dans la suite de cette séquence, les rôles ont pour fonction d'être un tremplin à l'élaboration de l'activité de jeu symbolique car ils s'actualisent dans des séquences d'activité de jeu symbolique un peu plus tard dans cette séance. Mais, pour l'instant, ils alimentent et orientent l'activité de construction avec intention de ce que le jeu symbolique, associé à ce thème, est susceptible de contenir.

\section{DEUXIÈME SÉQUENCE}

09:04:18 Aïe (prénom de l'enfant) le garage de pompiers va être à côté de toé!

09:25:27 Tiens, c'est là où les p'tites autos y rentrent. C'est là où les petits autos rentrent!

09:39:11 Aïe! Aïe! On dirait que ça serait inc le Monsieur-là qui a construit là là le poste de police.

09:51:04 Aïe! Lui là, ça serait le Monsieur quand y vont passer dans la barrière. Attend, plutôt, plutôt, bon.

Dans cette séquence de l'activité de construction, les deux rôles nommés "Monsieur-là a construit le poste de police", "Monsieur quand y vont passer la barrière" sont également des rôles fonctionnels car ici, ils traduisent de nouveau des éléments de connaissances de l'enfant quand au thème de construction exploité et mettent en place des éléments constitutifs possibles d'une trame symbolique. Cependant, dans cette séance, ces rôles ne seront pas réinvestis dans l'activité de jeu symbolique. Par contre, ce sont des éléments de connaissances qui supportent, alimentent et justifient l'activité de construction, puisque celle-ci ne se réalise pas à vide mais, sur des éléments du réel, de l'environnement, de ce que l'enfant connaît de son environnement et au rapport qu'il établit avec cet environnement.

- L'adoption de rôles et l'activité de jeu symbolique subordonnée à l'activité de construction

Dans l'activité de jeu symbolique proprement dite, l'adoption de rôles est envisagée selon l'activité soit subordonnée ou simultanée à l'activité de construction.

Les séances $2,4,6,7,8$ et 9 représentent un mode d'élaboration de l'activité de l'enfant au coin bloc, où l'adoption de rôles s'inscrit dans une activité de jeu symbolique subordonnée à l'activité de construction. 
La séance 2, enfants 1 et 2, présente quatre rôles: 1) personne non identifiée; 2) la sirène (rôle fantaisiste); 3 ) le roi (rôle périphérique) et 4) le géant (rôle de fiction).

Dans cette séance, le projet global de l'activité de construction est explicite au début, soit un "village de maisons". Différents projets secondaires et complémentaires s'inscrivent dans ce projet initial. Par exemple, même si au début de la séance une des enfants qualifie la production de "maison de châteaux", quelques minutes plus tard, la même enfant précise qu'il s'agit d'un château de sirène. Par la suite, la trame symbolique amorcée se greffe à ce thème du château de la sirène. Par contre, un ensemble de ces énoncés formulés s'inscrit dans une élaboration du cadre du thème.

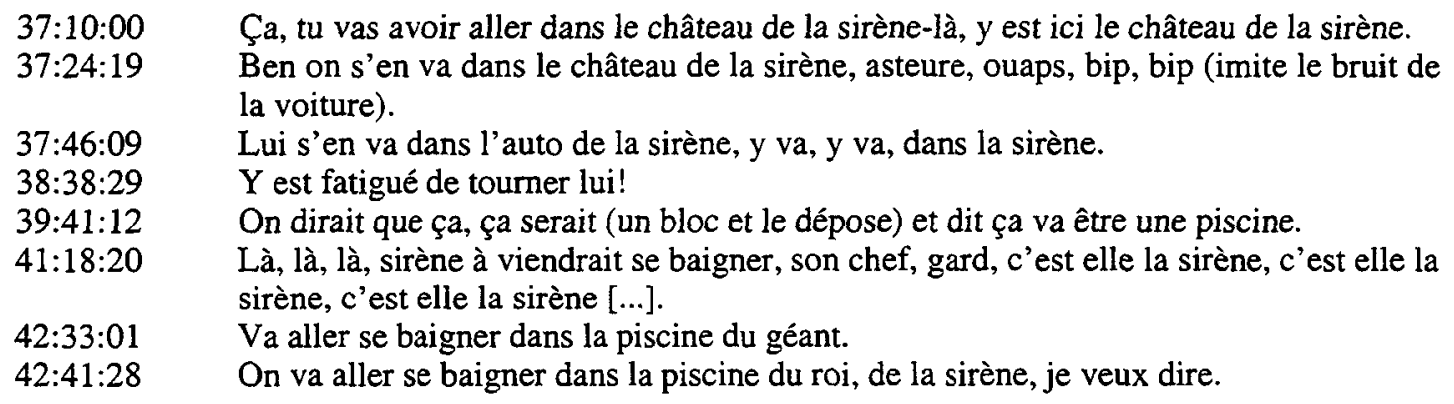

Deux énoncés s'inscrivent dans le scénario même de cette trame symbolique impliquant l'incarnation de personnages en gestes et paroles.

38:24:00 Oh ben, j'en tourné, je suis fatigué de tourner moé (l'enfant change de voix, donc on suppose qu'elle incarne un personnage).

43:12:26 La piscine est creuse, creuse, creuse, je me baigne même pas de flotte.

Dans cette séance, les enfants élaborent le cadre du thème de jeu et les différents rôles viennent soutenir les éléments de ce cadre. Les enfants ne jouent pas cette trame symbolique mais élaborent les éléments constitutifs de cette trame en mettant en place les éléments du cadre. Les enfants n'investissent pas les personnages, ils sont énoncés comme faisant partie intégrante de la trame symbolique du thème qui traduisent la connaissance des rôles susceptibles d'être joués dans ce thème. Ils élaborent le scénario, ils sont les scénaristes mais non les acteurs. 
Cependant les enfants 3-4, dans la même séance, présentent un projet global d'une maison qui est annoncé dès les premières secondes (00:15:00) et reprécisé à 01:39:02: "on va faire une maison, une maison pour rentrer dedans, hein, cette fois-ci on la fait grande, grande la maison." Plusieurs projets secondaires et complémentaires s'inscrivent dans l'élaboration du projet de la maison (barrière, balcon, plancher, porte). Dans cette dyade, certains rôles sont implicites et amènent les deux enfants à se coucher dans leur construction, à préparer des verres de jus et à les boire. Les enfants, dans cette trame, s'investissent eux-mêmes comme acteurs et les rôles sont rattachés à des actions exécutées au quotidien dans divers rôles familiaux.

Le premier épisode de cette trame symbolique s'inscrit en lien direct avec le projet global de la maison. En effet, en reconstituant certains espaces de la maison (cuisine, chambre), l'enfant reproduit certains comportements susceptibles de se manifester dans ces espaces. Ils traduisent sa connaissance et son expérience quotidienne de ce lieu.

Par la suite, un autre épisode s'amorce avec le choix de personnages de bois qu'ils distribuent un peu partout dans la maison. Un enfant propose: "Attention, nous autres on a un armée. Oh! caches-en d'autres ailleurs, y a des bombes qui éclatent" et la séance se termine sur ces paroles.

Par contre, le deuxième épisode de cette trame symbolique est déclenché par la présence de plusieurs personnages organisés dans les différents espaces de la construction et donne naissance à un thème nouveau où s'ajoutent l'armée et des bombes qui explosent. Comme cette trame est initiée à la fin de la séance, le temps disponible ne permet pas l'élaboration de cette nouvelle piste d'exploitation mais les rôles de soldats de l'armée représentés par les personnages de bois sont initiés de façon implicite.

À la séance 4, les enfants 3-4 présentent un projet global d'un bateau et ce, dès le début de la séance. L'activité de jeu symbolique subordonnée à l'activité de construction s'amorce après les 20 premières minutes de la séance. Le thème de cette trame symbolique "l'explosion de dynamites et de bateau" a pris sa source dans certains ancrages réalisés préalablement dans l'activité de construction qui se rapportent à: "un homme est mort;" "ici aussi je vais mettre des dynamites;" "dynamites et lancent des dynamites." Par la suite, les enfants-acteurs élaborent un scénario autour du thème: "faire sauter le bateau; attaquer les autres, explosion de l'eau, explosion des poissons, explosion de bateau." 
Cinq rôles émergent de cette trame symbolique, tous des rôles de caractère, stéréotypés et de fiction: tels celui de pirate, de matelot, d'esclave, de capitaine et de contremaître.

Ces différents rôles s'intègrent progressivement dans la trame symbolique. Ils sont initiés et joués par les enfants au fur et à mesure de l'élaboration du scénario. Ils traduisent également les connaissances des enfants en regard du thème exploité et le rapport qu'ils établissent avec ce qu'ils connaissent et comment ils le jouent.

À la séance 6, les enfants 2-3 présentent un projet global dès les premières secondes de la séance. Un enfant de cette dyade, après avoir construit son autoroute dans les cinq premières minutes de la séance, s'engage dans une trame symbolique, dans laquelle son monologue est constitué de deux personnages conducteurs et où ses actions entraînent les deux voitures dans diverses aventures sur l'autoroute.

Un deuxième épisode est amorcé quelques minutes plus tard à propos de l'idée de faire une ferme; et ainsi, il rejoint son partenaire qui est en train de construire un édifice. L'enfant sélectionne certains animaux dans le bassin d'animaux et monologue à partir de ces animaux. Par le biais du personnage singe, il escalade la construction de son partenaire de dyade, mais celui-ci n'accepte pas et il a peur que son compagnon démolisse tout. Finalement, il propose, par l'intermédiaire d'un quadrangle pour réaliser une pente, de monter dans son édifice pour faire circuler leurs voitures. Puis, un appel à l'aide est lancé pour signifier que la voiture est prise au piège et un dialogue s'enchaîne entre les deux partenaires sur "le fait d'être pris au piège et de la possibilité de se casser la gueule" et sur l'éventualité que l'édifice tombe et explose.

Cependant, un enfant veut rentrer dans l'édifice en ouvrant la porte secrète et il organise un système avec des petits blocs carrés pour faire fonctionner la porte. Par la suite, il décide que chacun a des garages et il distribue les étages de l'édifice. "Ä̈e! ça c'est mon garage, ça c'est mon garage, ces deux garages-là sont à moi. Ces deux garages, ces deux garages-là sont à toi" Mais comme il y a désaccord, une série de négociations et de propositions s'enchaînent. Finalement, après plusieurs négociations, ils prennent deux garages chacun, disposent les voitures et continuent de négocier les voitures, les garages, l'édifice et l'autoroute jusqu'à la fin. 
Dans ce dernier épisode de la négociation, il n'y a pas de véritable trame symbolique, ni de construction. Il y a cependant, différents éléments d'organisation d'un cadre de jeu autour des garages et des voitures et des propriétés de chacun. Ils établissent donc les bases de leur cadre de jeu, ils ne s'engagent pas à jouer un scénario, ils négocient le cadre de jeu.

À la séance 7, les enfants 1-2 présentent une activité de construction du projet global d'un château annoncé dès le début par l'un des partenaires de la dyade. Après 27 minutes, l'intention des enfants de réaliser leur projet, manifeste une activité de jeu symbolique mais il n'y apparaît pas d'idées précises et de trame symbolique. C'est plutôt une tentative d'exploration de différents possibles non en lien avec l'activité de construction, c'est-à-dire le château. De ces différents possibles, une seule trame semble émerger et refait surface dans les énoncés mais sans but précis. Ces possibles se retrouvent dans les énoncés suivants:

\begin{abstract}
"Prends les enfants" à 30:32:28 et partagés par son partenaire "Pis là je prends les enfants-là, pis là je suis allée dans l'autobus" et repris à 31:53:00 “Aïe! Toi tu serais le professeur. Aïe! Aïe! Débarque les enfants parce que c'est moi le professeur", ensuite l'idée de la classe et d'embarquer les enfants et de saluer Monsieur le professeur et à 32:48:09 "Venez-vous en, venez vous embarquer, venez les enfants."
\end{abstract}

Entre ces énoncés est initiée l'idée d'attaque, de vol, d'imitation du gorille, du tigre, d'autres animaux qui peuvent aider et l'idée des soldats. Donc, une seule trame symbolique très courte et très peu explicite précisant les rôles d'enfants et de professeur. Plusieurs énoncés apportant de nouvelles idées, sans lien, avec la trame amorcée, mais qui reprennent des éléments du discours des enfants de l'autre dyade: les attaqueurs, le voleur, le gorille, le tigre, les animaux et le soldat.

Cette lecture montre donc différents rôles éventuels, tous des rôles périphériques, qui ne sont pas joués, mais seulement initiés, possiblement dans un but d'exploration pour une éventuelle élaboration de trame symbolique. Donc exploration de possibles par l'initiation de divers rôles. 
À la séance 8 , les enfants 1 et 2 présentent un projet global d'une maison, annoncé dès les premières secondes par un partenaire de la dyade.

Quelques minutes plus tard, un des partenaires fait part du projet secondaire "là, on n'a pas le droit de rentrer, y'a une barrière". Cette proposition suggère à l'autre partenaire l'idée d'y installer deux gardes. Il apporte le bassin des animaux et initie le cri de chacun en les déplaçant dans l'espace. Donc, ce premier rôle est initié à la suite de l'activité de construction par le biais d'un projet secondaire de la barrière. À la suite de cette proposition, l'autre partenaire place les personnages de bois verts et jaunes un peu partout sur la production identifiée au départ comme une maison.

Un des enfants propose de faire une barrière d'animaux en plaçant ceux-ci à l'intérieur et en imitant leur cri. Simultanément, cette proposition amorce toute une classification des animaux: les plus malins, les pas méchants et les méchants, et cette activité donne naissance à une trame symbolique axée sur les animaux qui sont dans la guerre et qu'il faut placer dans une autre caverne. La classification se poursuit selon des critères de malins, pas malins, méchants, pas méchants, féroces, pas féroces. Une confusion se dégage pour l'un des enfants à propos de cette classification: "Lui, y'est pas malin, lui y'est pas malin, lui y'est pas malin, y sont féroces eux autres, ou ben sont malins?"

Dans cette trame symbolique, les enfants engagent leurs personnages animaux et leur personnage bonhomme de bois dans divers combats. L'idée de "zoo" émerge et les enfants adoptent encore toute une série de gestes et de paroles alimentant le thème.

Les rôles initiés et joués dans cette trame symbolique le sont à l'aide de personnages animaux ou des personnages de bonhomme de bois et ont un lien avec la trame qui se développe. Ils traduisent les connaissances des enfants au jeu et à mesure de l'élaboration de cette trame symbolique.

Ainsi apparaissent les rôles de roi des animaux en identifiant le lion, le rôle de patron en identifiant toujours le lion. À ce rôle, sont également identifiées ces différentes fonctions, "c'est malin, pis c'est le patron, il peut tuer les autres animaux, c'est le patron! Brrr" (imite le cri du lion) et attribue le rôle de chef au lion; un des enfants propose que c'est un château d'armée et introduit les rôles des ennemis et de guide. "Ça c'est un château d'armée. C'est toute des animals qui peuvent anéantir les ennemis, mais lui c'est pas 
un ennemi, c'est lui qui peut nous guider. Aïe! les ennemis. Hey (prénom de l'enfant)! Est-ce que tu sais où c'est? Aïe! Aïe! Est-ce que tu sais où est le derdermotus? Je voudrais leur dire quelque chose!"

Les enfants continuent leur trame symbolique et introduisent le rôle de chiens qui peuvent ressentir tout tout!

Rôles de deux animaux qui travaillent pour eux: l'éléphant et le chameau. Ils introduisent un rôle de voleur qui va aller se faire manger par les chiens. Introduisent le rôle de prisonnier en parlant des bonhommes de bois verts et jaunes.

Finalement, rôle de blessé (un personnage de bois) et un rôle de morts (personnages de bois).

En résumé, dans cette séance, les enfants initient et jouent plusieurs rôles par rapport aux animaux et aux personnages de bois qu'ils inscrivent dans la trame symbolique amorcée dès le début de l'activité de jeu symbolique. Tous ces rôles se chevauchent, s'intègrent et se complètent. Ils sont tous reliés entre eux.

Finalement à la séance 9, les enfants 1-4 annoncent la réalisation d'un bateau. Cette annonce se fait dans les premières minutes et ce projet global de bateau entraînera toute une série de différents épisodes (29) dans l'activité de construction et dans l'activité de jeu symbolique. Aucun rôle n'a été initié pendant l'activité de construction. Tous les rôles émergent de l'activité de jeu symbolique. Ces rôles sont: espion/voleur, attrapeur de poissons, blessé/docteur, ramasseur de billets, baigneur, pêcheur, lanceur de bombes, transporteur de bombes, matelot, des malades, l'armée. Plusieurs de ces rôles sont initiés en intercoordination, ce qui indique un souci de définir leur rôle en fonction d'une coordination.

L'adoption de rôles et l'activité symbolique simultanée à l'activité de construction

Les prochaines séances 3,7 et 9, représentent un mode de l'activité de l'enfant au coin bloc, où l'adoption de rôles s'inscrit dans une activité de construction et une activité de jeu symbolique avec simultanéité d'épisodes dans l'une ou l'autre de ces activités.

Dans la séance 3, l'activité de construction s'édifie sur le projet global d'un château où se greffent différents projets secondaires. 


\begin{tabular}{|c|c|c|}
\hline \multicolumn{2}{|c|}{ SEA3 ENF3-4 ACTIVITÉ DE CONSTRUCTION } & \multirow[b]{2}{*}{$\begin{array}{l}\text { Activité de jeu } \\
\text { symbolique }\end{array}$} \\
\hline $\begin{array}{l}\text { Activité de } \\
\text { construction }\end{array}$ & $\begin{array}{l}\text { Activité de construction } \\
\text { Simultanéité épisodique } \\
\text { de courte durée de l'activité } \\
\text { de jeu symbolique }\end{array}$ & \\
\hline 5 premières minutes & 29 minutes & 8 dernières minutes \\
\hline & 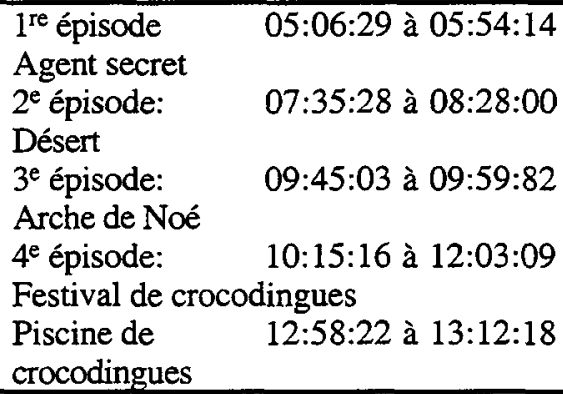 & \\
\hline & minutes & \\
\hline
\end{tabular}

Après cinq minutes d'activité de construction, un premier épisode est signifié par I'intermédiaire d'un rôle d'agent secret: " $Y$ 'a des agents secrets dans notre château, agent secret, agent secret, y'a des agents secrets. Y'a personne qui peut les connaître, qui peut les connaître. Il sait pas leur langue. Personne ne peut savoir leur langue." Ce premier rôle n'est pas joué mais discuté succinctement. Les enfants reviennent à leur construction et initient un deuxième épisode, cette fois-ci par l'intermédiaire d'une substitution d'objet (un bloc pour le désert). Dans cet épisode, aucun rôle n'est initié. Les enfants reviennent à leur construction, et ils initient (09:45:03) un autre épisode par l'intermédiaire d'un rôle "les croco" pour des crocodiles qui sont dans l'arche de Noé. Ils reviennent une minute à la petite salle du château et initient un autre épisode, soit "le festival des crocodingues" par l'intermédiaire du rôle des crocos et de la piscine des crocodingues par le biais d'une substitution d'objets, des courbes elliptiques, pour réaliser une piscine. Ils reviennent à l'activité de construction et ils jouent le rôle de nageur: "Bon je débarque du château (fait semblant de nager), blop! blop!" et son partenaire annonce que le bateau est terminé.

Après 20 minutes, un des murs de leur construction tombe et un enfant annonce une avalanche et un tremblement de terre. Ils reconstruisent en signifiant les différents espaces (cabine, toilette, plancher, cheminée, barrière) en lien avec le projet global de maison. Par la suite, un des enfants interpelle son partenaire en lui disant: "Aie Capitaine! $Y$ nous reste plus de bâtons. Capitaine, y nous reste plus de grande planche." Et l'autre accepte ce rôle de manière implicite en lui répondant: "Oui, y'en reste encore un peu." Dans cette activité de construction avec simultanéité épisodique de courte durée de l'activité de 
jeu symbolique, nous pouvons relever quatre rôles: agent secret, capitaine, nageur, crocodile, initiés mais très peu développés dans le contexte. Ils sont des amorces.

Nous avons vu précédemment que déjà dans l'activité de construction de la séance 7 dont le projet global est le poste de police, les enfants ont initié sept rôles différents qui traduisent des bribes de connaissances en lien avec le projet global.

\begin{tabular}{|c|c|c|}
\hline SEA7-ENF3-4 & Activité de construction & Activité de jeu symbolique \\
\hline Durée totale: 35 minutes & 11 premières minutes & $\begin{array}{c}\text { Simultanéité épisodique de courte } \\
\text { durée de l'activité de construction } \\
25 \text { minutes }\end{array}$ \\
\hline
\end{tabular}

Après 11 minutes d'activité de construction, un jeu symbolique s'amorce avec simultanéité épisodique de courte durée de l'activité de construction.

Au premier épisode, une série de rôles sont initiés en lien avec le projet global et concernent les rôles de policiers, de pompiers, un responsable des accidents, un voleur de diamants et de colliers, un voleur, de voleurs/de policiers, un patron des policiers/roi des policiers/bandits, un monsieur du garage/les accidentés, et finalement le rôle de patron.

Certains rôles sont annoncés en intercoordination, soit le rôle de voleur et celui de policier; ensuite le patron des policiers, le roi des policiers et le rôle du bandit, le monsieur du garage et ceux qui ont des accidents. Ici, la deuxième composante du jeu social ritualisé est mise en évidence. En effet, les partenaires se conduisent dans les voies particulières ritualisées dans lesquelles les actions sont intercoordonnées. Les enfants mettent en lumière leur souci d'établir des rôles complémentaires et intercoordonnés justifiant la fonction des rôles de l'un et l'autre. Ceci indique un stade plus avancé de l'interaction sociale des enfants et leur connaissance des rôles qui s'alimentent et se justifient mutuellement.

Plus tard, un des enfants annonce un accident et l'autre adhère implicitement à cet épisode en envoyant "un char de police". Donc, ici, des rôles complémentaires et intercoordonnés sont initiés pour mettre en scène cet épisode symbolique.

Après 24 minutes, les enfants effectuent une transformation symbolique du projet global "le poste de police en fourrure pour animaux". L'énoncé "Ça serait les animaux de 
la police" permet d'établir un pont entre la trame symbolique antérieure et l'amorce d'une nouvelle trame symbolique où s'enchaîne un dialogue entre les différents animaux qualifiés "des plus forts", associés au roi des animaux (cheval, zèbre, chameau, éléphant, gorille, lion) pour aider les policiers à accomplir leur travail.

24:44:14 Ouais, en tout cas, ça serait les animaux de police. Là, ça serait les animaux à les polices pour qui les arrêtent les autres, pour qui, les animaux c'est plus fort.

25:02:23 Animaux à les polices/voleurs.

Ben non, ça serait les animaux à les polices, qu'à, qu'à, pour pas que les voleurs les

27:08:08 Roi des animaux

27:18:16 Cheval $\rightarrow>$ roi des animaux.

Zèbre $\rightarrow>$ roi des zèbres.

Chameau $\rightarrow>$ roi des chameaux.

Éléphant $\rightarrow$ roi des éléphants.

Girafes $\rightarrow>$ roi des girafes.

Ours $\rightarrow$ roi des ours.

27:50:14 Gorille $\rightarrow$ roi des gorilles.

Éléphant $\rightarrow$ roi des éléphants.

Lion $\rightarrow$ roi des lions.

Panthère (gros chat) $\rightarrow>$ roi des gros chats.

28:29:27

29:06:20

$29: 24: 21$

Un voleur dans ton poste de police.

Tigre/voleur.

Voleur.

29:34:03 Tigre détruit le bureau de poste.

31:53:17 Enfants/animaux qui défendent les enfants.

32:11:21 Pompier.

32:36:20 Papa lion.

34:30:26 Compagnon/animaux pour qui nous aident.

34:54:09 Soldat.

35:04:27 Roi/princesse.

Dans ces énoncés, les enfants reprennent et continuent l'idée du voleur, idée initiée en lien avec le poste de police, ce qui indique qu'il y a chevauchement entre la fonction initiale de la production du poste de police et la transformation symbolique de cette production en fourrière pour animaux. Il y a également chevauchement et intégration des rôles dans le scénario du voleur et des animaux qui défendent. Les enfants poursuivent leurs premières idées de scénario, qui constituent les premiers ancrages symboliques dans les nouveaux épisodes initiés. Finalement, vers la fin de la séance, le rôle de soldat, de roi et de princesse apparaissent mais sans développement.

En résumé, dans cette séance, 39 rôles sont initiés dont sept dans l'activité de construction et 32 dans l'activité de jeu symbolique. Évidemment, plusieurs de ces rôles ne sont pas joués. Ils ont dans l'ensemble un lien avec le projet global de construction 
initial, par exemple le poste de police qui, au plan symbolique, se transforme en fourrière pour animaux et où les animaux ont comme fonction d'aider les policiers pour défendre, protéger et arrêter. Les scénarios s'enchaînent, s'alimentent et se complètent dans une trame symbolique qui évolue et qui est initiée par "bribes" dans l'activité de construction. L'ensemble de ces épisodes de jeu symbolique se caractérise par l'annonce de cette possibilité sans déroulement exhaustif. Ces différents rôles sont amorcés soit par l'intermédiaire de l'annonce même du rôle, soit par du matériel ou des accessoires, ou soit par des gestes de l'enfant.

Finalement, la séance 9 s'amorce par l'annonce du projet global de construction par l'énoncé suivant: "On fait un château", et par la construction d'un garage connexe à partir du plancher.

\begin{tabular}{|l|c|c|c|}
\hline SEA9-ENF2-3 ACTIVITÉ DE CONSTRUCTION & \multicolumn{1}{|c|}{} \\
\hline Activité de construction & $\begin{array}{c}\text { Activité de construction } \\
\text { Simultanéité épisodique } \\
\text { de longue durée } \\
\text { de l'activité } \\
\text { de jeu symbolique }\end{array}$ & Activité de construction & $\begin{array}{c}\text { Activité } \\
\text { de jeu symbolique }\end{array}$ \\
\hline 15 premières minutes & 6 minutes & 1 minute & 22 dernières minutes \\
\hline \multicolumn{2}{|c|}{22 minutes } &
\end{tabular}

Un premier épisode laisse apparaître une activité de jeu symbolique qui s'annonce à 15:13:14 par: "Aie! ça va être ton canon pour détruire ton bateau". Pendant cette séquence de six minutes, une trame symbolique s'élabore autour du canon et de la poudre à canon pour détruire le bateau. Par la suite: "Ah oui, sloup! J'ai attrapé une baleine béluga". Donc, ici, l'enfant initie un rôle de pêcheur de baleine. Trois autres rôles sont identifiés par rapport au contexte de lancer des bombes pour détruire le bateau. Les rôles de quelqu'un qui vient sur notre bateau, de contrôleur et de lanceur de bombe sont initiés par le thème du lancement des bombes. La trame symbolique qui découle de ces différents rôles est très peu explicite, elle se construit plutôt en chapelet.

\section{- L'organisation à quatre}

La séance 5 présente le seul type d'organisation à quatre de cette étude et ce, pour l'activité de construction et l'activité de jeu symbolique. 


\begin{tabular}{|c|c|c|}
\hline SEA5-ENF1-2-3-4 & $\begin{array}{c}\text { Activité de construction } \\
\text { Simultanéité épisodique de courte durée } \\
\text { de l'activité de jeu symbolique }\end{array}$ & $\begin{array}{c}\text { Activité } \\
\text { de jeu symbolique } \\
\text { Simultanéité épisodique } \\
\text { de courte durée de } \\
\text { l'activité de construction }\end{array}$ \\
\hline Durée totale: 42 minutes & 28 minutes & 14 dernières minutes \\
\hline
\end{tabular}

D'une durée de 42 minutes, la séance se déroule majoritairement sur un mode d'activité de construction (28 minutes) qui constitue l'activité de base, avec simultanéité épisodique de courte durée de l'activité de jeu symbolique.

Les 14 dernières minutes se déroulent sur un mode d'activité de jeu symbolique avec simultanéité épisodique de courte durée de l'activité de construction.

Il y a donc chevauchement et alternance des deux types d'activités. Cependant, les quelques insertions d'activités symboliques et la trame symbolique des dernières minutes sont peu exhaustives et complexes.

- Les substitutions d'objets

En ce qui concerne la première composante structurale: les substitutions d'objets, 32 substitutions simples d'objets sont effectuées pendant les 28 premières minutes de l'activité de construction et sept substitutions d'objets se sont réalisées à l'intérieur de scénario, soit pendant les 14 dernières minutes (voir tableau 4.10).

Cette séance est recoupée selon divers épisodes qui sont amorcés par le biais de la production en cours, par des rôles éventuels, par une combinaison de la production et du rôle éventuel, par une onomatopée signifiant le cri d'un animal ou encore par un geste. 
Tableau 4.10

Les différentes substitutions d'objets dans l'organisation à quatre

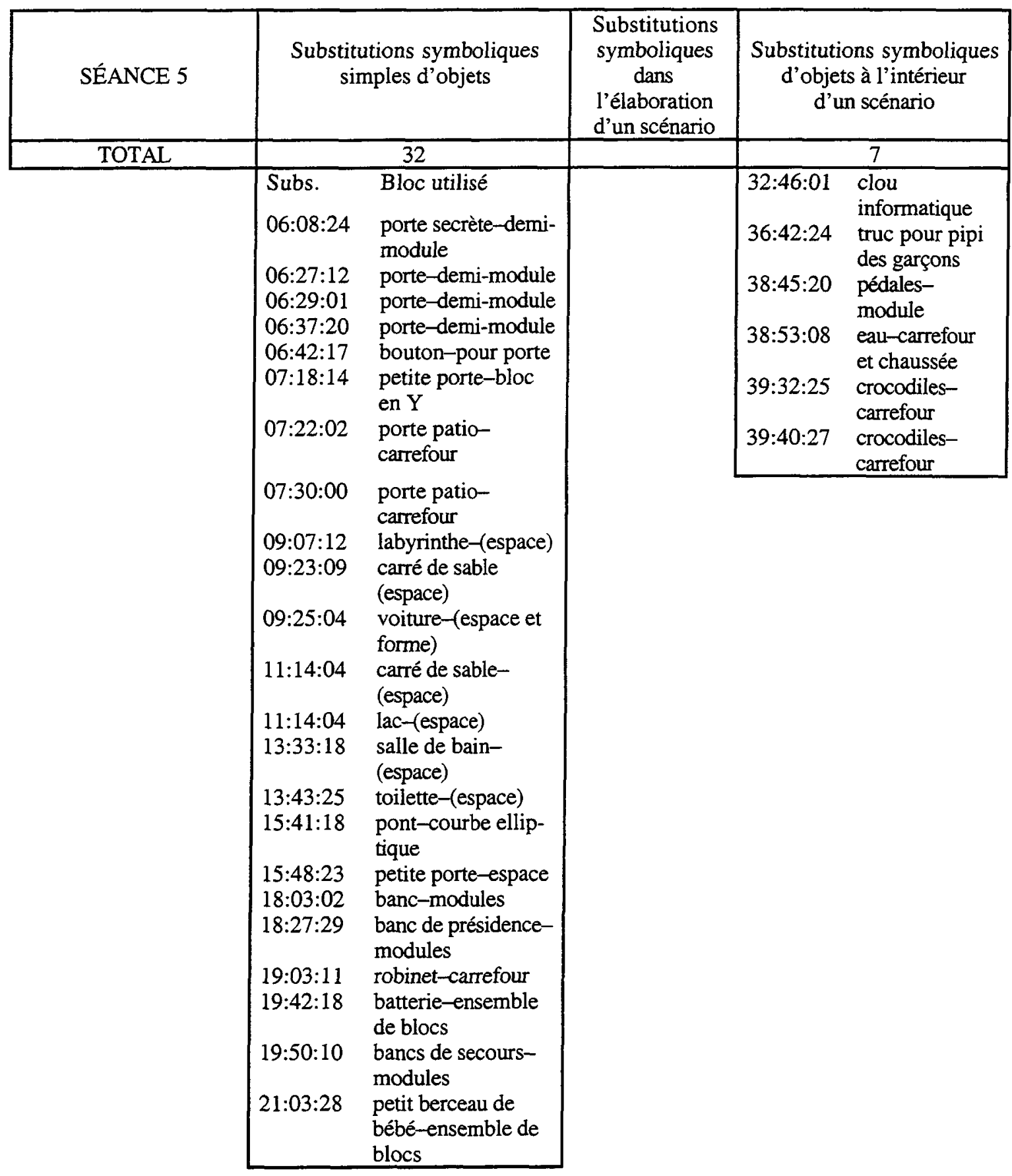


Tableau 4.10 (suite)

\begin{tabular}{|ll|}
\hline $\begin{array}{r}\text { Substitutions symboliques } \\
\text { simples d'objets }\end{array}$ \\
\hline $21: 14: 17$ & $\begin{array}{l}\text { piano de secours- } \\
\text { ensemble de blocs }\end{array}$ \\
$21: 33: 10$ & $\begin{array}{l}\text { système d'alarme- } \\
\text { ensemble de blocs }\end{array}$ \\
$22: 42: 10$ & salle de gymnasti- \\
& que-espace \\
$22: 48: 24$ & lac-espace \\
$24: 57: 19$ & poignard-bloc \\
$26: 25: 25$ & marteau-demi-arche \\
& romaine \\
$26: 25: 25$ & clou-triangle \\
$27: 38: 29$ & cheminée-cylindre \\
\hline
\end{tabular}

\section{- L'adoption de rôles}

Le projet global annoncé est une maison et l'association des quatre enfants se soude après quatre minutes.

À partir de la production d'un château, un premier rôle est mentionné, soit l'étranger et les voleurs d'or. Le rôle de voleur revient, suite à la production du projet secondaire des bancs de sécurité, et ce rôle est reformulé à cinq reprises. Plus tard, un nouveau rôle apparaît, celui de capitaine. Par la suite, un enfant annonce: "On a fini notre maison, là, on joue-tu?".

Successivement, les enfants initient les rôles de pêcheur de baleine, de pianiste, de capitaine, de joueur de guitare, de pêcheur, de nageur, de plongeur, de nageur, qui donnent naissance à des épisodes plus ou moins longs. Cette séance donne lieu à 12 rôles dans l'élaboration de plusieurs épisodes.

Dans cette deuxième question de recherche, les différentes composantes structurales ont été rendues observables par l'analyse de la communication verbale des enfants. L'observation des enfants, en prenant compte de cette communication, s'enrichit d'informations inestimables et substantielles sur les intentions, les actions et les interactions des enfants dans l'activité de construction et l'activité de jeu symbolique. Se priver de cette communication verbale, c'est comprendre cette dynamique de jeu que très partiellement. 
En tenir compte, c'est s'inscrire dans une démarche de compréhension globale de l'activité de l'enfant avec le matériel bloc. Au fil des analyses, la communication verbale est donc apparue comme un témoin des plus significatifs et des plus importants pour comprendre les modes d'organisation et d'interaction des deux activités des enfants avec les blocs.

Ainsi, nous abordons la troisième question de recherche, qui s'inscrit dans la réflexion des interactions sociales qui sous-tendent l'activité de construction et l'activité de jeu symbolique.

\subsection{Troisième question de recherche: les interactions sociales}

Dans le contexte de la classe maternelle, l'activité de construction et l'activité de jeu symbolique suscitées par le matériel bloc sont de plus en plus des activités qui se réalisent à plusieurs partenaires, donc des activités qui s'organisent en situation de collaboration.

De ce fait, il apparaît qu'un ensemble d'éléments essentiels dans la structure de l'activité de l'enfant avec le matériel bloc se situe sur le plan interactionnel, c'est-à-dire sur le plan des interactions sociales entre enfants. Ces mêmes éléments influenceraient et détermineraient l'activité de construction et l'activité de jeu symbolique simultanée ou subordonnée à l'activité de construction. En posant la prémisse que les interactions sociales jouent leur rôle dans le développement cognitif et social de l'enfant, on peut se demander, quel type d'interactions sociales émergent de l'activité de l'enfant avec le matériel bloc?

Dans un premier mouvement, nous nous attendons pertinemment à ce que les enfants qui choisissent le coin bloc s'adonnent à une activité dite de construction. Cette activité peut alors, dans ce cas-ci, se réaliser à deux, trois ou quatre enfants et déjà, selon l'agencement des enfants, cette première activité crée un réseau d'interactions entre les enfants lequel favorise la réalisation d'un projet collectif de construction. Il est alors légitime de se demander:

Quelles sont les interactions sociales qui émergent de la dynamique de l'activité de construction au coin bloc? Quelles sont celles qui favorisent davantage la réalisation d'un projet collectif de construction? 
Dans un deuxième mouvement, l'observation des enfants au coin bloc et la revue des écrits nous rappellent que l'enfant de 5-6 ans qui fréquente la classe maternelle se situe dans une période de son développement particulièrement féconde sur le plan symbolique. À cet âge, il appréhende le monde qui l'entoure par un mode symbolique qui se traduit fortement dans son activité ludique. Ce mode symbolique d'appréhension alimente et teinte la majorité des actions et des interactions des enfants. Il devient en quelque sorte la trame de fond sur laquelle se tisse le mode de développement de l'enfant de 5-6 ans et, par conséquent, la manifestation du jeu symbolique s'observe aisément dans les différentes activités de la classe maternelle et spécifiquement dans l'activité de l'enfant au coin bloc. Donc on peut ici encore une fois s'interroger:

Quelles sont les interactions sociales qui émergent de la dynamique de l'activité de jeu symbolique au coin bloc? Quelles sont celles qui favorisent davantage la réalisation d'un projet collectif de jeu symbolique?

Depuis le début des années 80, les interactions sociales sont devenues une préoccupation majeure pour de nombreux chercheurs. Ceux qui se sont intéressés et qui continuent de s'intéresser au jeu de l'enfant n'y font pas exception, car les différentes situations de jeu sont pour eux des terrains qui permettent d'investiguer le système des interactions sociales.

Partant du principe de base que toute activité impliquant deux personnes et plus met en branle un réseau de communications et d'interactions entre elles, toute situation où l'enfant est en présence d'autres enfants va entraîner nécessairement un réseau de communications et d'interactions en dépendance au but évident qui les réunit.

Dans une situation, plus spécifiquement de jeu, les enfants sont amenés à partager avec les autres un espace et un matériel où se crée tout un système de dynamiques sociales. Les situations dans lesquelles l'enfant se retrouve dans ce réseau complexe de dynamiques sociales sont nombreuses et diversifiées tout au cours de sa vie. Cependant, dès le début de son existence, l'enfant est déjà plongé, dans ses premières relations avec la mère et son entourage immédiat, dans tout un système d'interactions où chacun des membres de cette dynamique agit, réagit, interagit et interréagit en fonction de ce qu'il est, de ce que l'autre est, de ce qu'il fait et de ce que l'autre fait. Dès son plus jeune âge, il amorce l'élaboration et la construction de tout un échafaudage d'actions, de réactions et 
d'interactions en fonction des personnes, du contexte, de ce qui se dit, se sent, se fait, etc. Ces différentes constatations ne font que traduire une infime partie de la complexité de ces dynamiques sociales.

L'enfant, dans les diverses situations de sa vie, apprend à entrer en relation avec les différentes personnes, pairs et adultes, ce qu'on exprime par "se socialiser peu à peu”. Ainsi, les nombreuses expériences de vie de l'enfant contribuent à l'acquisition et à la consolidation de diverses compétences cognitives et sociales qui lui permettent de devenir de plus en plus autonome, responsable et compétent dans les différentes relations qu'il établit avec l'autre. Il est donc difficile de séparer en entités différentes les compétences cognitives et sociales qui appartiennent spécifiquement à l'une ou l'autre de ces expériences; ce serait comme essayer de trouver laquelle vient avant l'autre.

Cependant, même si les compétences ne peuvent s'associer de manière privilégiée à telle ou telle situation, il est possible d'examiner, dans une situation particulière, en l'occurrence l'activité de l'enfant au coin bloc, la manière dont les enfants s'y prennent pour entrer en relation, partager, confronter, négocier et pour réaliser des activités ensemble, visant un but commun. Ces façons de faire ont certes leur point d'ancrage dans la façon dont les enfants ont appris et continuent d'apprendre à entrer en relation avec leur milieu familier et dans la façon dont ils ont appris à confronter, transformer, modifier, ajuster, et adapter leurs différents modes d'actions, de réactions et d'interactions, avec de nouvelles expériences et de nouvelles personnes (pairs et adultes). Ainsi, le coin bloc à la maternelle fournit à l'enfant un lieu, un espace et un matériel qui lui offrent l'opportunité d'affronter et de confronter sa réalité des modes d'interactions avec d'autres enfants.

Suite à ce questionnement, nous nous sommes donc interrogée sur les formes précises d'interactions sociales des enfants dans la situation du coin bloc à la maternelle.

\subsubsection{Les négociations interpersonnelles dans l'activité de construction et dans l'activité de jeu symbolique}

Comme indiqué précédemment, la troisième question de recherche concerne les interactions sociales susceptibles de favoriser la réalisation d'un projet collectif de l'activité de construction et de l'activité de jeu symbolique dans le coin bloc. 
Le filtre des interactions sociales dans la dynamique de l'activité de l'enfant avec le matériel bloc peut mettre en lumière différents éléments susceptibles d'apporter des éclairages multiples de l'activité de l'enfant dans cette aire de jeu. Cependant, à la lumière des recherches positionnant les interactions sociales et le jeu symbolique, il est possible d'examiner divers aspects de ces interactions sociales par le biais du partage de significations, des connaissances psychosociales, des relations interpersonnelles, etc. Des choix ont été faits en fonction de ce qui apparaissaient être des éléments essentiels à la compréhension et l'explication du questionnement de cette troisième question de recherche. Évidemment, sous une montagne de données qui offre une multitude de possibles, faire des choix implique tout un renoncement à des aspects qui semblent fournir des pistes de compréhension. Cela risque aussi de lancer la chercheuse sur des pistes d'éparpillement, il devient donc difficile d'organiser la compréhension qui finirait par ne plus vouloir rien dire.

Ainsi, nous avons établi nos choix en fonction des négociations interpersonnelles et des moments de conflits, deux éléments qui apparaissent donner des pistes intéressantes à l'analyse. Ces modes de fonctionnement des individus dans les dynamiques interpersonnelles risquent de proposer des situations où chacun des partenaires a la possibilité de se positionner dans le rôle que l'activité en cours exige, mais aussi dans celui de la personne elle-même, dans cette dynamique.

C'est à partir du "verbatim" des différentes séances d'observation que nous tenterons de dégager les diverses stratégies interactives que les enfants déploient pour assurer la viabilité d'une activité partagée de construction et de jeu symbolique. Nous dégagerons aussi le respect de soi-même comme personne dans cette dynamique, car l'un et l'autre étant souvent imbriqués de manière très implicite. Évidemment, ce choix s'inspire de convictions intuitives qui, par la suite, ont été soutenues théoriquement par des réflexions qui mettent en lumière la construction, dès un très jeune âge, de stratégies interactives qui permettent le dénouement productif de différentes situations.

À l'âge de trois ans, les processus qui conduisent à l'acceptation réciproque moiautrui s'avèrent fort complexes et ressemblent, en leurs mécanismes, à la négociation interpersonnelle, telle qu'elle a été mise en évidence au sein des relations familiales (parents-enfants-couples). [...] Le problème que pose la discrimination entre l'accord ou le refus d'un message ainsi que la perplexité créée par des messages ambigus sont les mêmes que ceux qui nous font souffrir, nous, adultes. Nous non plus ne sommes parfois pas capables de sortir de certaines impasses, impasses très 
proches de celles rencontrées par ces jeunes enfants: ne plus savoir qui dit quoi, et pourquoi l'accord que l'on vient de sceller est brusquement remis en question par des comportements ressentis comme une sorte de négation de l'évidence, négation de soi-même et de la relation à l'autre. Nous, adultes, sommes amenés à nous interroger sur l'histoire de la relation, sur notre capacité à la comprendre, sur notre sensibilité et sur les stratégies qui ont permis à des (pseudo-) accords de se transformer en un désaccord profond. A la base de ces équivoques, nous trouvons souvent la peur d'approfondir l'exploration interpersonnelle, peut-être pour ne pas courir le risque d'essuyer un refus ou un conflit intrapsychique. (Bonica, 1990, p. 148-149)

De plus, les moments conflictuels permettent de mettre en évidence les stratégies interactives mises en branle par les enfants afin de revenir sur un terrain d'accord. D'abord, en s'assurant que le terrain du conflit est commun, c'est-à-dire qu'il n'y a pas d'ambiguïté sur la nature du conflit. Par la suite, en se demandant quelles sont les stratégies déployées pour trouver ou retrouver un terrain commun d'entente où chacun est respecté autant sur le contenu de l'activité que sur le plan personnel. Cet examen des moments de conflits devrait nous amener sur la piste des interventions pédagogiques de l'adulte, en l'occurrence l'enseignant, qui fait montre de l'importance de laisser vivre aux enfants les situations conflictuelles plutôt que de les évincer, de les éviter. Ainsi, les enfants pourront développer des stratégies interactives qui permettent de faire face à la situation problématique, de la confronter avec tous les risques impliqués dans une confrontation et, par la suite, d'élaborer des stratégies qui permettent de retrouver un équilibre autant dans la dynamique de jeu que dans la dynamique personnelle et interpersonnelle.

Habituellement, on cherche à éviter aux enfants les situations difficiles et on se préoccupe de renforcer leur capacité à occulter les conflits internes plutôt que de les laisser expliciter et maîtriser. L'abandon du jeu, le refus explicite et la découverte partagée avec autrui de sa propre conflictualité interne peuvent être une expérience formidable pour apprendre à maîtriser le déséquilibre intra et interpersonnel tout en encourageant la recherche d'une réciprocité toujours plus différenciée qui est indispensable pour parvenir à partager un accord profond avec autrui. [...] C'est tout à fait le principe de l'adaptation fonctionnelle soulignée à plusieurs reprises par Wallon (1949) et qui caractérise les êtres humains, dès le début, pour garder l'interdépendance nécessaire à la survie et à l'exploration des autres milieux non humains. Grâce à cette disposition, les enfants nous démontrent qu'ils peuvent aborder et résoudre des situations paradoxales qui restent toujours très difficiles même chez les adultes. (Stamback, Sinclair, 1990, p. 144)

\section{- Quelques éléments théoriques aux négociations interpersonnelles}

La notion de négociation renvoie à la définition de Bonica quant à "une forme particulière de communication sur le désaccord" (Stamback et Sinclair, 1990, p.113). Ces tra- 
vaux de recherche tentent de préciser qu'il s'agit d'une forme de communication qui vise à rétablir un accord sur le contenu du jeu de fiction ou un accord sur l'acceptation réciproque de chacun en tant que personne. Ces analyses, en dégageant la transition entre les moments d'entente et les moments de conflit, permettent de mettre en évidence la contribution des négociations interpersonnelles pour une meilleure compréhension du rôle des interactions sociales entre enfants dans le processus de différenciation moi-autrui. Elles s'inscrivent également dans l'éclairage de la signification des jeux de fiction dans la régulation des rapports interindividuels entre partenaires et permettent de voir en quoi la fiction est source de conflits ou, au contraire, un moyen de les résoudre.

Les éléments théoriques qui servent de base à ces analyses sont issus des travaux de Wallon (1949) et de Bateson (1956). Dans l'élaboration de la différenciation moi-autrui, Wallon insiste sur l'importance du social dans la construction du moi et de la connaissance. Pour lui,

le milieu social fonctionne tantôt comme activateur de conflits, tantôt comme élément modérateur permettant leur résolution et ceci, selon une alternance entre participation mimétique et distanciation ou encore entre fusion et différenciation. L'enfant est vu dans la double fonction d'acteur et de spectateur. (Wallon, 1949: voir Bonica, 1990, p. 114)

Cette double fonction témoigne de la conscience de soi comme personne qui, à la fois, est influencée par la présence d'autrui et peut à son tour influencer le comportement d'une autre personne.

La différenciation moi-autrui se construit selon différentes étapes. La première se constitue par des contrastes émotionnels (plaisir-déplaisir) et par des rapports interindividuels opposés (exiger-subir). Les enfants, dans les différentes situations, vont expérimenter, tour à tour, ces deux positionnements par des jeux d'alternance. Progressivement, les enfants identifient les différents acteurs de la situation et les rôles de chacun dans cette situation.

Wallon (1949) identifie qu'à partir de trois ans, les enfants parviennent à comprendre que les situations peuvent modifier les rapports interpersonnels et les rôles assumés par les partenaires, et que c'est principalement par le rôle de cette opposition rapports interpersonnels/rôles assumés que s'élabore, peu à peu, la conquête de la différenciation moi-autrui. 
Toujours selon cet auteur, cette opposition s'explique:

surtout par l'incapacité d'intégrer complètement la non-permanence des rôles et des attitudes selon les situations. Cette opposition brutale représente un pas en avant par rapport aux jeux d'alternance, car les deux pôles acteur-spectateur, au lieu d'être seulement situés en deux individus différents, peuvent être maintenant présents dans la même personne. Mais une telle intégration est encore instable: pour se construire, elle a besoin de passer par autrui qui, tel un miroir, permet en même temps l'opposition et l'intégration des deux pôles complémentaires. (Wallon, 1949: voir Bonica, 1990, p. 115)

Les travaux de Bateson (1956), pour leur part, mettent en lumière les deux niveaux de la communication. Selon cette perspective, chaque acte de communication traduit à la fois des éléments d'information sur le contenu et l'objet de l'échange, et sur la qualité de la relation interpersonnelle. C'est un message "métacommunicationnel" parce qu'il dit des choses sur la façon dont le contenu doit être interprété dans le contexte interactif. Des ambiguittés peuvent donc surgir entre ces deux niveaux de la communication lorsqu'il y a une contradiction entre la communication verbale et la communication non verbale qui l'accompagne. L'accord dans les échanges des partenaires suppose la compréhension simultanée des deux niveaux de la communication et engendre un déroulement de plus en plus cohérent, harmonieux et productif de l'activité commune. Le désaccord dans les échanges des partenaires suppose une incompréhension des messages, et il peut donc s'agir d'une contradiction entre les deux niveaux de communication, et pour que l'équilibre soit rétabli, la discussion doit se réaliser au même niveau de communication. L'indication la plus probante de cette contradiction entre les deux niveaux de communication s'observe dans les épisodes de conflit et de négociation, là où se pose avec le plus d'acuité le problème de la différenciation entre le contenu et la forme de relation, et là où la négociation sur les rapports interpersonnels émergent avec la spécificité la plus forte, même si, selon les apparences, les partenaires semblent continuer de discuter sur le contenu.

Selon cet auteur, le message relationnel peut véhiculer trois ou quatre types d'informations possibles: "l'accord (tu as raison); le désaccord (tu n'as pas raison); la dévalorisation (je ne te reconnais pas comme émetteur, j'ignore ce que tu dis, je ne te reconnais pas comme un être qui peut avoir des pensées, intentions, émotions qui peuvent influencer autrui); la double injonction (j'exprime des sentiments contradictoires qui t'empêchent de comprendre si je suis d'accord ou pas)" (Bateson, 1956: voir Bonica, 1990, p. 116). 
La négociation à propos de la relation interpersonnelle assure la défense de l'intégrité interne du moi chez chaque partenaire. Donc, chaque réplique dans les épisodes de conflit et de négociation contribue à préciser les caractéristiques de la relation et à développer la coordination des significations à travers les transformations apportées aux contenus.

La dimension interpersonnelle est toujours présente, mais plus évidente et visible dans les moments de conflit et de négociation témoignant de la contradiction entre ces deux niveaux de communication. Ces moments de conflit peuvent survenir à tout moment, témoignant à chaque fois d'un déséquilibre à propos de l'interprétation du contenu comme à propos de la définition de la relation interpersonnelle. Lorsque le déséquilibre investit la sécurité émotionnelle, l'objet de la négociation se déplace sur la relation interpersonnelle afin de rétablir l'équilibre.

Le désir de rester ensemble entraîne l'interférence des stratégies de modification de la situation interactive avec les stratégies de coordination autour du contenu. Il s'agit pour les partenaires de discriminer la valeur d'accord et de désaccord qui reflète la capacité de chacun à s'accorder sur le niveau de communication. Discute-t-il alors du contenu ou de la relation?

Dans cette perspective théorique, les conflits et leurs résolutions apparaissent comme particulièrement révélateurs des mécanismes régulateurs des relations interpersonnelles. Plus spécifiquement, pour les modalités d'analyse des données de notre recherche, nous reprenons les mêmes bases théoriques de Wallon et de Bateson pour réaliser l'analyse des séances d'activité de construction et d'activité de jeu symbolique, des concepts de base qui constituent les éléments fondamentaux de la cinquième composante structurale du jeu symbolique social: la structuration du jeu.

\subsubsection{La mise en forme des données}

1. L'analyse des données de la troisième question de recherche a nécessité une mise en forme particulière des données afin de dégager les interactions sociales. Pour chacune des séances, les énoncés verbaux et non verbaux ont été regroupés sur quatre colonnes verticales, une colonne pour chaque enfant. La transcription du "verbatim" sur quatre colonnes a permis de faire ressortir toutes les interactions sociales des enfants autant celles 
générées par les enfants d'une même dyade ou groupe que celles générées entre les enfants de dyades ou groupes différents.

Chaque réplique a été numérotée dans la séquence temporelle d'apparition et une flèche d'indication assure le mouvement des répliques entre les enfants d'une même dyade et les autres enfants.

2. Une première lecture de la séance permet l'identification des trois moments distincts du déroulement de chaque séance: le positionnement initial, l'élaboration de l'activité de construction et l'élaboration de l'activité de jeu symbolique.

3. Après l'identification des trois phases, on procède à l'identification des épisodes, des séquences et des événements à l'intérieur de la phase de l'activité de construction et de l'activité de jeu symbolique. Ce deuxième découpage montre les interactions sociales qui constituent les épisodes entre les enfants d'une même dyade ou groupe et entre les enfants de dyades ou groupes différents.

Dans cette étude, chacun des termes se définit comme suit: une phase présente les moments successifs importants de l'évolution du déroulement de l'activité de l'enfant avec le matériel bloc. Trois phases sont identifiées: le positionnement initial, l'activité de construction et l'activité de jeu symbolique.

Un épisode dans l'activité de construction regroupe l'ensemble des répliques et des actions sur le matériel d'une dynamique impliquant des échanges à propos des éléments de construction liés au projet global de l'activité de construction. Chaque projet global nouveau apparaissant dans le déroulement de l'activité de construction constitue un nouvel épisode.

Un épisode dans l'activité de jeu symbolique regroupe l'ensemble des répliques et des actions sur le matériel d'une dynamique impliquant des échanges à propos des éléments de ce jeu autant dans l'activité de construction que dans l'activité de jeu symbolique. 
Une séquence est constituée de l'ensemble des répliques et des actions sur le matériel générées par les membres d'une même dyade ou du même groupe. Elle découpe les épisodes en divers plans.

Un événement est constitué de l'ensemble des répliques et des actions sur le matériel qui mettent en évidence les interactions sociales générées par les enfants n'appartenant pas à la même dyade ou au même groupe. Il inclut également, l'intervention spontanée de l'enseignante et l'intervention sollicitée par les enfants. Les événements découpent les séquences en divers faits.

Ainsi, pour chaque séance, on retrouve trois phases de base essentielles du déroulement de l'activité de l'enfant avec le matériel bloc: des épisodes identifiant des éléments de construction liés aux différents projets globaux de l'activité de construction et des éléments de jeu symbolique liés au thème de l'activité de jeu symbolique; des séquences regroupant les interactions sociales intra-dyade ou intra-groupe; et finalement des événements regroupant les interactions sociales inter-dyade ou inter-groupe.

Le tableau 4.11 illustre le découpage en phases, épisodes, séquences et événements de chacune des séances de jeu. 
Tableau 4.11

Découpage en phases, épisodes, séquences et événements de chacune des séances de jeu

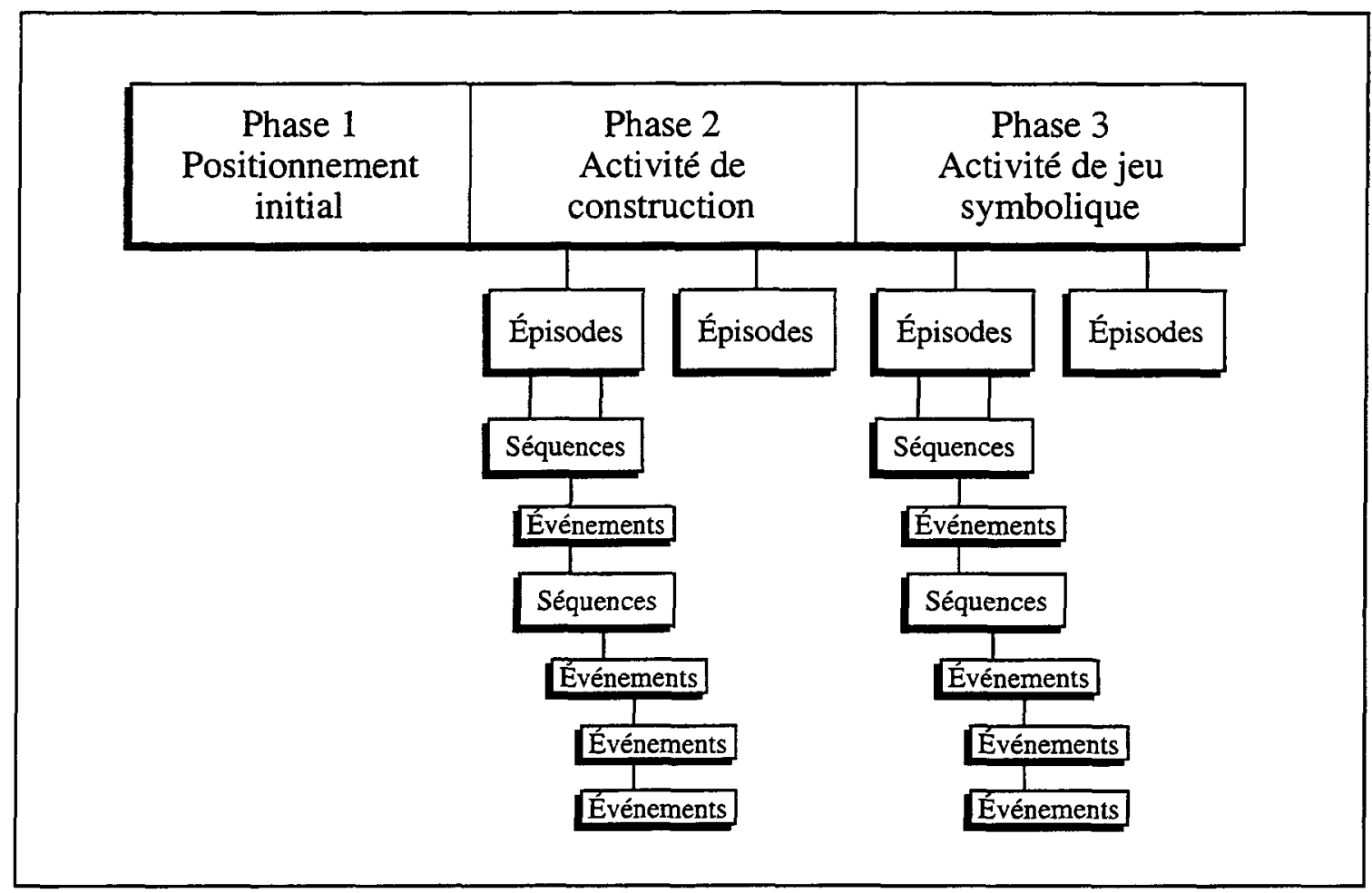

4. Une fois la structure de la séance organisée, le contenu de chaque réplique est analysé afin de faire ressortir l'objet, le sens et la stratégie interactionnelle utilisée par l'enfant. L'exemple suivant présente le mode d'analyse de chacune des répliques de la séance.

Ex: Stéphane, on fait-tu une maison?

Objet $\quad \rightarrow$ Intention de projet global de construction à un partenaire potentiel.

Sens $\quad \rightarrow$ Demande explicite à un partenaire par le biais d'une intention de projet global, une maison.

Stratégie $\rightarrow$ Interpelle directement un partenaire potentiel.

interactionnelle Proposition d'intention de projet global par questionnement. 
Deux sources ont permis d'élaborer une liste de codes des répliques pour déterminer des catégories susceptibles de rendre compte de la nature du contenu de chaque réplique afin de dégager les stratégies interactives utilisées par les enfants qui favorisent la réalisation d'un projet collectif de construction de jeu symbolique.

Ces sources s'inspirent du modèle des catégories mixtes préexistantes, doublé des catégories induites, tel qu'élaboré par L'Ecuyer (1990). Dans ce modèle, le chercheur utilise les diverses sources des différentes étapes de la recherche et actualise les différentes étapes de sa démarche. En fait, il utilise les nombreux matériaux de ses lectures théoriques, de ses intuitions et de ses réflexions pour élaborer une liste de catégories. Ce modèle est qualifié de modèle ouvert, car il permet de tenir compte de toutes les particularités susceptibles d'entraîner des modifications substantielles dans l'organisation des catégories préexistantes et surtout, ouvre la possibilité d'en ajouter plusieurs autres. Les catégories préexistantes constituent un point de départ, un cadre de référence, et laissent une grande ouverture sur le matériel analysé, car il tient aussi compte des catégories qui émergent de l'analyse.

Dạns un premier temps, une liste de verbes et de noms témoignant des diverses stratégies a été élaborée, à partir de notes théoriques et de stratégies observées dans différentes recherches recensées, ayant pour but d'investiguer les interactions sociales et le jeu symbolique. Cette liste a permis d'identifier l'ensemble d'un vocabulaire spécifique à l'identification du contenu de chaque réplique afin de dégager des pistes éventuelles de sens en fonction des stratégies interactives utilisées par les enfants.

Cette liste de verbes et de noms a permis d'élaborer une deuxième liste, mais cette fois-ci de codes, traduisant la nature du contenu interactionnel de chaque réplique.

5. Par la suite, pour chacune des séances, nous avons relevé les séquences et les événements témoignant d'un désaccord, soit des négociations interpersonnelles impliquées dans ces moments de transition ententes/conflits.

L'analyse de ces séquences et de ces événements devrait:

- faire mieux comprendre la transition entre les moments d'entente et les moments de conflit dans l'activité de construction et l'activité de jeu symbolique; 
- permettre de contribuer à une meilleure connaissance du rôle des interactions sociales entre enfants intra-dyade et inter-dyade dans le processus différenciation moi-autrui;

- permettre d'éclairer la signification de l'activité de construction et de l'activité de jeu symbolique dans la régulation des rapports interindividuels entre partenaires et de voir en quoi ces deux activités sont sources de conflits ou, au contraire, moyens de les résoudre.

\section{- Les données relatives aux interactions sociales}

Un premier relevé concerne la quantité totale de matériel verbal produit et le nombre effectif de sujets s'étant exprimés ainsi que le nombre d'épisodes, de séquences et d'événements générés par cette production verbale.

Trois mille trois cent cinq $(3,305)$ répliques ont été produites par 36 enfants durant les neuf séances d'observation. Ces répliques ont constitué une cinquantaine d'épisodes dans l'activité de construction et dans l'activité de jeu symbolique, générant une cinquantaine de séquences et une centaine d'événements.

- Première phase: le positionnement initial

L'analyse de l'enchaînement des différentes répliques dans l'étude du positionnement initial permet de dégager: le temps pris par les enfants pour réaliser cette première phase importante dans la poursuite de l'activité de l'enfant avec le matériel bloc; les différentes stratégies interactives déployées par les enfants dans la recherche d'un partenaire et dans l'établissement d'une intention de projet global de l'activité de construction.

- Le temps dans le positionnement initial

La recherche d'un partenaire pour constituer une dyade, une triade ou un groupe de quatre se réalise dans les cinq premières minutes de la séance. 


\begin{tabular}{|c|c|c|c|c|c|c|c|c|c|}
\hline Séances & 1 & 2 & 3 & 4 & 5 & 6 & 7 & 8 & 9 \\
\hline Tempsenminutes & 4 & 3 & 0,75 & 3 & 4 & 5,5 & 0,75 & 1 & 6 \\
\hline
\end{tabular}

- Des stratégies interactives d'association des partenaires dans ce positionnement initial.

L'analyse des répliques de la phase de positionnement initial laisse apparaître différentes stratégies déployées par les enfants afin de s'associer avec un partenaire potentiel.

Différents enfants de la séance initient des tentatives d'association: 1) un même enfant interpelle différents enfants pour l'association jusqu'à l'acceptation d'un de ces enfants; 2) un même enfant initie auprès d'un seul partenaire potentiel et, à son refus, abandonne toute autre tentative; 3 ) un enfant n'initie aucune tentative d'association mais accepte une proposition d'association d'un autre enfant; 4) un enfant n'initie aucune tentative d'association et refuse toute proposition d'association.

Dans cette phase de positionnement initial, différentes stratégies interactives d'association sont déployées dans la recherche active d'un partenaire de jeu.

Globalement, 12 stratégies interactives d'enfants initiateurs ont été relevées dans ces neuf séances et sont regroupées selon la forme dominante de la stratégie. La présentation de ces stratégies est accompagnée d'exemples de répliques représentatives de la stratégie.

1. Des stratégies d'interpellation:

a) Interpelle l'associé potentiel par son prénom. Ex: Cédrik! Cédrik! (2) (SEA1-ENF3);

b) Interpelle par son prénom et proposition par questionnement explicite de l'intention de projet global au "on". Ex: Stéphane! On fais-tu une maison? (1) (SEA2-ENF4)

c) Interpelle par son prénom et information explicite de projet global et secondaire. Ex: Jean-Benoît! Aie! Notre château y'a pas de porte! (57) (SEA9-ENF2)

d) Interpelle par son prénom et demande explicite d'aide avec marque de possibilité d'association à l'intention de projet global. Ex: Aie! Aie! Elizabeth! Elizabeth! Veux-tu m'aider à faire mon château? (SEA6-ENF3) 
2. Des stratégies de verbalisation:

a) Verbalisation explicite de l'intention de projet global ou secondaire au "moi-je". Ex: Ah ben moé, je fais une belle bâtisse. () (SEA4-ENF3); Non, non, c'est mon auto, c'est mon auto, c'est pas ton auto (3) (SEA4-ENF?); Je n'ai juste besoin d'un! On a besoin d'une porte (SEA9-ENF1); Ben moi, je vas faire un bateau (SEA1-ENF1)

b) Verbalisation explicite de besoin de matériel au "on". Ex: On a besoin de ces affaires-là.

3. Des stratégies d'offre d'aide:

a) Offre explicite d'aide. Ex: Jonathan! Je vais t'aider à faire le poste de police. (SEA1-ENF2); N'as-tu encore besoin?; N'as-tu encore besoin? (SEA7-ENF1)

b) Offre d'aide explicite par questionnement avec marqueur de possibilité. Ex: Est-ce que je peux t'aider? (1) (SEA3-ENF3)

4. Des stratégies d'évaluation:

a) Évaluation du projet global de l'autre. Ex: Hé! Ça va tomber. (14) (SEA1ENF2)

b) Évaluation positive du projet global de l'autre - stratégie de séduction. Ex: Aie! est belle ton autoroute. (16) (SEA6-ENF4)

5. Des stratégies d'attirance:

a) Attire l'attention sur l'intention de projet global. Ex: Regarde, je fais un beau château-là. (19) (SEA4-ENF3); Aie! regarde-moi. Mon bateau à moteur. Comment est faite. Y'a un beau plancher. (29) (SEA4-ENF2); Y est beau hein! Mon royaume! Y est en train de se faire bâtir. (30) (SEA6-ENF4)

6. Des stratégies de conseil:

a) Donne des conseils avec marqueur de cause. Ex: Il faut que tu le tournes toute parce que... (15) (SEA1-ENF2)

7. Des stratégies d'avertissement:

a) Avertissement avec marqueur de cause et constat. Ex: Faites attention parce que c'est fragile. (16) (SEA1-ENF2); Non, parce que si vous en mettez des trop longs, ça va toute tomber! Y faut n'en mettre des petites comme ça! (SEA9-ENF1); On serait mieux d'en prendre des plus petits si on veut pas qui défasse!. Aie! on peut faire un bateau. (SEA9-ENF1) 
8. Des stratégies propositionnelles:

a) Proposition explicite de l'intention de projet global au "on". Ex: On va faire une belle maison. (10) (SEA1-ENF2); On va faire un village de maisons. (5) (SEA2-ENF2); On se fait une belle bâtisse. (1) (SEA3-ENF3); On fait une belle auto. (2) (SEA4-ENF3); Aie! Y'en a des comme ça aussi, hein! Ça va être gros hein!. Y'en a gros des plaquettes comme ça pour faire un bateau comme l'autre fois! On fait un bateau comme l'autre fois. Aie! C'est un château, un château bateau. (SEA5-ENF2); On va faire, on va faire un poste de police. (SEA7-ENF1); On fait une maison à deux. (SEA8-ENF1) (Simon); Attends un petit peu! Ça on va mettre là, là avant de mettre les petits blocs là, on va mettre ça, là. Mets les petits blocs là. Regarde deux comme ça. (SEA8-ENF1); On va faire une belle maison à nous deux. (SEA8-ENF4); Aie! On peut construire nos gros châteaux. On va faire notre gros château. Attends non! la planche y faut la mettre là. (SEA9-ENF1); Aie! On va faire un garage. (43) (SEA9-ENF3)

b) Réitère la proposition explicite de l'intention de projet global au "on" avec ajout d'information. Ex: On va faire une belle maison avec beaucoup d'étages. (12) (SEA1-ENF1); On va faire une maison pour rentrer dedans, hein! Cette fois-là on la fait grande, grande, grande la maison. (14) (SEA2-ENF4)

c) Réitère la proposition explicite de l'intention de projet global au "on" avec ajout d'information spécifique et demande d'accord. Ex: On va faire une belle maison avec trois étages, OK. (13) (SEA1-ENF1)

d) Proposition explicite mais confuse d'intention de projet global avec marqueur de possibilités au "on" puis au "je". Ex: Aie! Jean-François! On pourrait faire un bateau. Oh! Ben moi, moi je fais un beau château. (SEA6-ENF3)

e) Proposition explicite d'action par le biais du matériel accessoire au "on". Ex: On va aller chercher les autos. (9) (SEA1-ENF3); Attends! Je vas aller chercher un autre planche! Tiens Jean-Benoît! (SEA9-ENF1)

f) Proposition explicite par questionnement d'intention de projet secondaire au "on" avec marqueur de possibilité. Ex: Ah! Est-ce qu'on peut faire notre toit? (7) (SEA4-ENF ); Aie! C'est la porte, la porte, y faut faire la porte! (SEA9ENF1)

g) Proposition explicite d'intention de projet secondaire au "on". Ex: On va faire le plancher. (12) (SEA2-ENF4); Mais on fait le plancher. (SEA9-ENF5); Aie! On fais-tu un jeu? On fais-tu un foyer? Regarde. (36) (SEA9-ENF3)

h) Proposition explicite d'action sur le matériel avec marqueur d'obligation. Ex: Aie! Faut mettre des grandes, des grandes plaquettes avant, avant de mettre ça. (SEA5-ENF1)

i) Proposition explicite d'emplacement au “on". Ex:On va faire ça là! (11) (SEA2-ENF4); Aie Manon! On, moi je joue avec Jonathan dans le "boutte" de l'atelier. (SEA7-ENF2) 
9. Des stratégies de demande:

a) Demande explicite par le biais du matériel. Ex: L'enfant place ses planches de construction avec celles d'un projet de construction en cours d'un autre enfant. (SEA1ENF2)

b) Demande explicite de matériel avec une proposition explicite d'intention de projet global au "nous" sous forme directive. Ex: Ah! Donne-moi z'en. Fais la maison avec nous. (7) (SEA1-ENF1); Donne-moi le bloc. (16) (SEA2-ENF2); Aie Jonathan! Vas-tu me chercher un bois! (SEA9-ENF3)

c) Demande explicite par questionnement avec marqueur de possibilité d'association. Ex: J'peux-tu faire avec vous? (SEA5-ENF3)

d) Demande explicite d'information par questionnement sur son intention de projet global. Ex: Qu'est-ce que tu fais? (SEA6-ENF2); Par où qu'on va rentrer? (SEA6-ENF2)

10. Des stratégies d'invitation:

a) Invitation explicite sous forme directive. Ex: Aie! Viens! Je construis. (SEA9ENF2)

11. Des stratégies de directive:

a) Directive par questionnement explicite de l'intention de projet. Ex: Là! Là, tu m'attenras, on va faire quoi? (9) (SEA2-ENF4); Qu'est-ce qu'on pourrait faire? (20) (SEA4-ENF4)

12. Des stratégies d'annonce:

a) Annonce d'intention de projet global avec proposition d'action. Ex: Moi, je fais un bateau, Jean-Benoît veux-tu embarquer dans mon bateau? (23) (SEA4-ENF2)

Simultanément à ces différentes stratégies interactives d'association, les données révèlent diverses formes d'acceptation et de refus.

Les acceptations se formulent de la façon suivante:

a) Accepte en reprenant à son compte l'intention de projet global. Ex: On va faire pareil. (7) (SEA2-ENF1); Bon, ben moi aussi. (8) (SEA4-ENF2);

En offrant son aide et en demandant et en proposant: Ex: Un beau château, je peux t'aider, qu'est-ce qu'on pourrait faire. OK on pourrait mettre ça. (SEA4-ENF4); Jonathan! Je vas t'aider à faire le poste de police. (4) (SEA7-ENF4). En ajoutant un 
qualificatif: Ex: Notre maison va être plus belle. (20) (SEA2-ENF4). Et en proposant un projet global complémentaire: Ex: Pis un château aussi! Hein Émilie! Hein! (16) (SEA5-ENF4). En combinant les propositions des deux projets globaux: Ex:Bateau, château-bateau. (17) (SEA5-ENF1)

b) Accepte en proposant un emplacement pour la réalisation de l'intention de projet global. Ex: On n'a pas le droit, garde on va le mettre là. (10) (SEA2-ENF3); Aie Manon! On, moi je joue avec Jonathan dans la boîte de l'atelier. (2) (SEA7ENF4); Ah oui! Ben non! Ici! (41) (SEA8-ENF3)

c) Accepte. Ex: Ouais c'est vrai! (SEA3-ENF3); Ouais. (25) (SEA4-ENF3); Ouais. (13) (SEA 5-ENF2); Ben oui. (62) (SEA5-ENF4); On est quatre, garde, un, deux, trois, quatre [en pointant chaque enfant]. (64) (SEA5-ENF3); Y'a deux garçons, y'a deux filles. (66) (SEA5-ENF2); Ouin! (3) (SEA8-ENF2); On est pas quatre, on est trois [1 (Jean-François), 2 (Cédrik), 3 (Véronique)] 2-2 (63) (SEA5-ENF1)

d) Accepte en attirant l'attention sur le projet global initié par l'autre et en proposant un projet secondaire. Ex: Aie! Regarde moé, mon bateau comment y'est faite. Y'a un beau plancher. (29) (SEA4-ENF2); Hein! Avant de faire la cheminée. (4) (SEA5-ENF4). En proposant du matériel bloc Ex: Hop! Y'en manque un! (5) (SEA8-ENF2); Y'en manque pas juste un, y'en manque plus! (6) (SEA8ENF2)

Les refus se formulent de la façon suivante:

a) Un retrait implicite par le biais du matériel. Ex: L'enfant déplace les planches qu'il avait agencées à la construction d'un autre enfant.

b) Ignore ou ne répond pas à l'interpellation, la verbalisation, l'offre d'aide, l'évaluation, le conseil, l'avertissement, la proposition, la demande, l'invitation, les directives ou l'annonce.

c) Refuse par une contre-proposition de projet global. Ex:Aie! Non! On s'en fait un bateau. (24) (SEA4-ENF4); Non! Non! Non! On fait un château. (21) (SEA9ENF3); Non! Pas comme ça! (39) (SEA8-ENF3); Aie! Non! Non! Touche pas. (9) (SEA9-ENF3)

d) Refuse avec un critère extérieur. Ex: Non toé t'es pas dans nôtre! Peggy est pas dans notre rue hein! T'es pas dans notre rue, t'es dans rue à Cédrik. (8) (SEA7-ENF4)

e) Retrait explicite par le biais de l'emplacement du projet global. Ex.: Aie! Colle pas ton bateau à côté de mon char! Moi je vas mettre un bloc, moi, je vas mettre un bloc. Pis y va arriver plein sur ton bateau. (32) (SEA4-ENF2)

f) Refus explicite. Ex: Non! (2) (SEA6-ENF1). En annonçant son intention de projet global. Ex: Non! Je fais une autoroute. (7) (SEA6-ENF3); Ben moi, je vas faire un bateau! (SEA9-ENF1) 


\section{- Deuxième phase: l'élaboration de l'activité de construction}

La phase de construction se poursuit après le positionnement initial. Cependant, les éléments de l'activité de construction tels que les propositions d'intention de projet global et de projet secondaire, les offres d'aide à construire, les propositions d'utilisation du matériel accessoire, les propositions d'emplacement, les demandes de matériel et les invitations à participer à la réalisation du projet global sont les bases de négociation des différentes stratégies interactives déployées par les enfants dans la recherche d'un partenaire potentiel. Il apparaît donc que l'activité de construction est l'objet central des premières négociations de l'activité de l'enfant avec le matériel bloc.

Les interactions sociales dans la phase du positionnement initial ont pour but la recherche d'un partenaire éventuel et ont comme objet de négociation les éléments de l'activité de construction qui sont des éléments de persuasion pour l'association de partenaires.

Dans l'évolution du déroulement du jeu de l'enfant avec le matériel bloc, les interactions sọciales générées dans la deuxième phase ont comme but la réalisation partagée de l'intention de projet global et de projets secondaires. Elles ont comme objet de négociation les éléments de l'activité de construction nécessaires à l'agencement du matériel bloc pour la représentation externe de la production envisagée. Dans cette deuxième phase, les stratégies interactives déployées par les enfants sont les mêmes qu'à la phase du positionnement initial, mais leur contenu se déplace sur les éléments de construction qui rendent possible l'élaboration partagée du projet de construction.

Pour illustrer le déroulement de l'activité de construction et l'enchainement des répliques traduisant les interactions sociales de cette activité, nous rapportons des extraits des neuf séances observées en dégageant le contenu des stratégies interactives des enfants afin de mettre en évidence, dans un premier temps, les éléments de l'objet de négociation dans cette deuxième phase et, dans un deuxième temps, les éléments de l'objet de négociation dans les séquences présentant un désaccord intra-dyade ainsi que les événements de désaccord inter-dyade. 


\section{- Les éléments de l'objet de négociation de la deuxième phase}

Dans la séance 2, l'association entre Simon et Cédrik est conclue dans les deux premières minutes. Les deux garçons discutent de l'esthétique de leur maison.

SIMON

02:13:06

Notre maison va être plus belle. (20)

02:13:09

Notre maison va être ben plus belle. (21)

02:28:13

Woh! Moi pis Cédrik on va faire une grande pour rentrer dedans.

(En plaçant les planches les unes à côté des autres pour faire le plancher (23)
CÉDRIK

02:19:27

Nous autres, on pourrait te rentrer, toute, toute.

02:33:11

Mais on va rentrer dedans. (24)

02:38:24

Tiens on va l'installer ici. (26)

02:42:24

Viens on va l'installer ici.

(En plaçant une planche à côté des autres pour faire le plancher)

Par la suite, l'activité de construction se poursuit pendant plus de 30 minutes. À l'intérieur de cette activité, on ne dénote qu'un seul épisode, LA MAISON, élaboré à partir de l'intention de projet global initialement annoncé à la phase de positionnement.

Dans cette activité de construction, les interactions sociales relevées se regroupent sous trois grandes catégories.

La première catégorie présente les interactions à propos de la réalisation du projet global et du ou des projet(s) secondaire(s) pour:

- informer;

- reprendre à son compte la proposition de l'autre, l'intégrer et la combiner à une nouvelle proposition;

- proposer un emplacement de réalisation; 
- repositionner le projet global;

- demander l'accord du partenaire;

- proposer des projets secondaires;

- évaluer le projet de construction en cours;

- anticiper les résultats possibles;

- justifier, reprendre et expliquer la proposition;

- questionner sur des actions éventuelles;

- constater l'avancement des travaux;

- argumenter une nouvelle proposition;

- demander de l'aide;

- offrir son aide;

- verbaliser sa procédure de construction.

La deuxième catégorie concerne les interactions à propos du matériel bloc pour:

- choisir le matériel;

- agencer le matériel;

- proposer du matériel;

- offrir du matériel;

- évaluer la quantité de blocs nécessaires;

- évaluer les besoins de matériel;

- transporter le matériel;

- demander de l'aide pour le transport du matériel;

- demander de l'aide pour l'agencement du matériel;

- proposer des agencements du matériel;

- donner des directives sur les types de blocs;

- exécuter une directive;

- contre-proposer une idée de matériel;

- verbaliser la procédure d'agencement du matériel;

- répartir le matériel;

- partager les transformations symboliques du matériel.

La troisième catégorie définit des interactions plus générales pour:

- consolider leur association;

- évaluer leurs compétences;

- évaluer la production en cours; 
- informer sur les modalités de fonctionnement de l'atelier:

- à propos de l'espace à utiliser;

- à propos de la circulation dans l'aire;

- à propos des règles du jeu;

- formuler un commentaire.

Cet extrait de la séance 2 est représentatif des diverses interactions observées dans l'activité de construction de toutes les séances et pour tous les enfants.

De plus, dans cette dyade, Cédrik semble occuper le statut de "meneur de jeu" et Simon, le statut d'exécuteur de décisions. Dans l'ensemble de la séance, on retrouve les différentes directives de Cédrik quant au transport du matériel et son agencement.

Dans l'activité de construction, le mode de fonctionnement à l'intérieur de la dyade démontre donc des habiletés, des compétences et des stratégies qui favorisent un déroulement cohérent et harmonieux, de plus en plus productif dans l'activité en cours. Un respect, une écoute des idées, des propositions et des contre-propositions à propos de la réalisation du projet global et du matériel qui permettent un dénouement intéressant de l'activité en cours.

Ce qu'on dénote aussi dans le déroulement de la séance, c'est que chacun a un intérêt pour jouer avec l'autre, et utilise des stratégies pour conserver et consolider l'association, et pour contribuer à un dénouement intéressant et satisfaisant du jeu où chacun semble trouver son profit.

L'observation du déroulement de cette séance fait ressortir le partage, pour les deux enfants, du même niveau de communication. Les différentes répliques se situent sur le plan du contenu de l'activité de construction (idées, projet global, projet secondaire, accessoires, emplacement, transformations symboliques, transformations matérielles) et ces répliques ne semblent pas laisser apparaître d'ambiguité sur le plan de la qualité de la relation. Les statuts des deux enfants sont implicites mais clairs et chacun reconnaît et est reconnu dans ce statut. 
- Séquence de désaccord intra-dyade

L'extrait suivant représente une séquence de la dyade où des désaccords peuvent survenir quant à l'objet même de la construction.

$[\ldots]$

\section{CÉDRIK}

\section{JEAN-MICHEL}

20:43:07

Aie! On fait-tu la cabine? (290)

20:45:18

Prête-moi tes blocs. (293)

20:53:00

Je fais la cabine moi. (294)

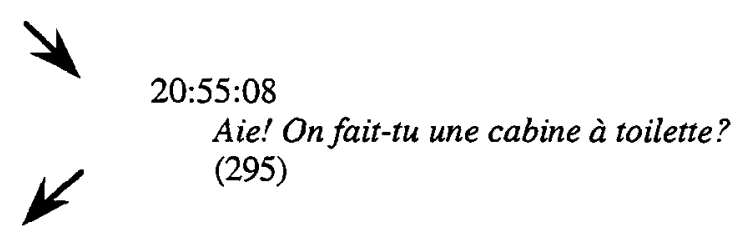

20:57:00

Non, pas de cabine, des toilettes. (296)

21:07:19

Non, tu sais la cabine. (299)

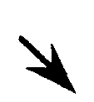

20:59:02

Oui, une cabine à bol. On fait une barrière. (297)

21:05:02

Ça va être le salon, ça va être la cuisine. (298)

21:16:23

(Il se lève et les blocs tombent)

21:23:23

On va faire une cheminée. (302)

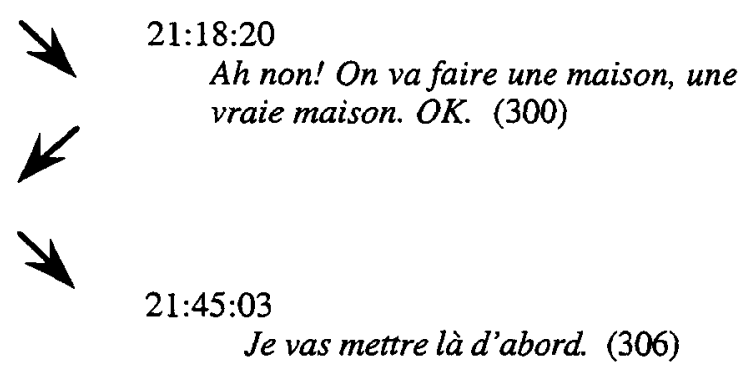

21:58:20

Je n'ai faite une, une cheminée. (En marchant sur la construction vers Jean-Michel) (307) 
22:17:00

Viens-t'en ici. (308)

$22: 21: 17$

Viens-t'en ici. (309)

22:22:15

(Explique à Jean-Michel ce qu'il veut faire mais Jean-Michel n'est pas d'accord puis il défait sa cheminée) (310)

22:41:03

Non, on fait pas ça. On fait une vraie maison. (311)

23:07:05

On fait une vraie maison. (314)

23:07:25

(Place un bloc le long du plancher en restant assis sur le plancher)

23:18:23

(Commence une nouvelle construction à côté de leur plancher)

23:20:25

Bonne idée!

(Donne des blocs à Jean-Michel)

23:44:00

(Apporte des planches à Jean-Michel. Donne les planches à Jean-Michel)

\section{3:56:15}

Nous autres, on va faire plein de châteaux, tiens (en donnant des planches à Jean-Michel) (321)

24:01:22

Aie Capitaine! Y nous reste plus de bâtons (quadrangles) (322)

24:07:06

Capitaine, y nous reste plus de grandes planches. (323)

23:19:16

Ben là, on va construire une vraie maison. Va me chercher les planches, comme ça (quadrangles).

23:47:08

Prête! Ah! OK.

23:54:11

Donne-moi z'en toujours. 
Les premières répliques de cette séquence s'amorcent par une proposition de projets secondaires. Cette proposition est reprise et refusée par le partenaire. À ce moment-là, une série de différents possibles à propos du projet secondaire de construction est explorée (cabines de toilettes, cabine à bol, barrière, salon, cuisine) pour revenir à la construction d'une vraie maison et pour enchaîner avec une cheminée et par la suite, à la proposition de construction d'une vraie maison.

À première vue, l'objet de négociation semble être une entente sur le choix du projet secondaire à construire; cependant, une analyse plus en profondeur laisse supposer que le désaccord ou l'ambiguitté d'une non-entente repose sur la qualité de la relation interpersonnelle.

Ici, le statut de décideur dans cette séquence, soit Cédrik, ne semble pas d'emblée accepté par son partenaire puisque des négociations s'engagent d'abord sur le type de cabine et, par la suite, sur tout autre projet secondaire. Par contre, le désir de rester ensemble est certainement dominant car plusieurs stratégies de persuasion sont déployées pour convaincre l'autre de la pertinence de son projet. La piste de négociation à propos du statut de chacun commence à apparaître lorsque Jean-Michel accepte partiellement la proposition en la modifiant quelque peu. Le refus par le changement d'idée de la proposition de Cédrik sème un doute sur son statut de décideur.

L'ensemble des répliques qui précèdent cette séquence révèle clairement et explicitement le statut de meneur de jeu de Jean-Michel qui donne des directives pour le transport et l'agencement du matériel, et le statut d'exécuteur de Cédrik qui accepte ce rôle qu'il va confirmer lui-même dans une de ses répliques lorsqu'il attribue à Jean-Michel le rôle de capitaine (réf. répliques 322, 323). Ainsi, l'acceptation des deux rôles permet de diminuer l'ambiguité et le jeu peut se poursuivre puisque les rôles dans la relation interpersonnelle sont explicitement définis et acceptés par chacun.

- Événement de désaccord inter-dyade

La séance 2 de l'activité de construction présente 15 événements générés par des interactions inter-dyade. La moitié de ces événements sont initiés par Audrey, les autres sont initiés par Cédrik ou Simon mais suscitent dans tous les cas de vives réactions d'Audrey. 
Voici la liste et les propos de chacun de ces événements.

\begin{tabular}{|c|c|c|}
\hline Événement & Objet de l'interaction & Initiateur de l'événement \\
\hline $1^{\mathrm{er}}$ & Statut des enfants & Cédrik \\
\hline $2^{e}$ & Compétences de chacun & Audrey \\
\hline $3^{e}$ & Infiltration dans le projet de l'autre dyade & Audrey \\
\hline $4^{e}$ & À propos du matériel et de sa répartition & Simon \\
\hline $5^{e}$ & Limites de l'aire & Cédrik \\
\hline $6^{\mathrm{e}}$ & Observation / Compétence & Simon \\
\hline $7^{e}$ & Matériel et casier de rangement & Audrey \\
\hline $8^{e}$ & Observation d'ingérence & Audrey \\
\hline $9 \mathrm{e}$ & Observation / Évaluation de la construction & Audrey \\
\hline $10^{e}$ & Commentaires et mise en garde & Audrey \\
\hline $11^{\mathrm{e}}$ & À propos de la répartition du matériel & Audrey \\
\hline $12^{\mathrm{e}}$ & Directives sur les modalités de fonctionnement & Simon \\
\hline $13^{\mathrm{e}}$ & Enfant d'une autre aire & Audrey \\
\hline $14^{\mathrm{e}}$ & À propos du matériel & Cédrik \\
\hline $15^{\mathrm{e}}$ & À propos du matériel accessoire & Simon \\
\hline
\end{tabular}

- Quelques éléments de rappel de la phase du positionnement initial

Dans la première phase de cette séance, rappelons qu'une association de partenaires se réalise en moins de trois minutes. Au début de la séance, Cédrik questionne directement Stéphane en proposant son intention de projet global: "Stéphane! On fait-tu une maison?" Mais Stéphane ne répond pas verbalement, il prend une grande planche et deux petits blocs et continue seul, sa construction amorcée.

Audrey annonce explicitement son intention de projet global en utilisant le "on". Stéphane ne répond pas directement à sa proposition mais dit: " $\mathrm{C}$ 'est comme ça" en plaçant son bloc de la même manière qu'Audrey. Donc, il y a une certaine forme d'acceptation implicite de la proposition d'Audrey quant à l'intention de projet. À la suite de la réponse de Stéphane, elle propose l'idée de "faire pareil". 
À ce moment, Cédrik intervient et donne son avis: "Ouais, mais pour faire une maison, ça prend du temps" et il propose implicitement de s'associer à Audrey: "Là, là tu m'attenras, on va faire quoi?" en la questionnant sur des éventuels possibles, donc en tenant compte de son opinion. Mais Audrey n'apporte pas de réponse à cette question. À ce moment, Simon en profite pour s'infiltrer en disant: "On n'a pas le droit, garde, on va se mettre là, oh non!'. Donc, il propose un emplacement dans l'aire pour réaliser un projet de construction avec Cédrik. Cédrik répond: "Peut-être que vous allez...". Par cette intervention, il semble annuler la proposition faite à Audrey ou, du moins, à ne plus en tenir compte et continue son intervention: "On va faire ça là" en parlant de lieu à Simon.

Dans la dyade Audrey-Stéphane, Audrey occupe le statut de meneur de jeu et Stéphane celui d'exécuteur. Cependant, cette association est claire et ne cause pas d'ambiguité sur les statuts. En fait, tout au long de l'activité de construction, Stéphane semble bien poursuivre la réalisation de ses intentions. À quelques reprises, il signifie son association à Audrey car il accepte implicitement les différentes propositions et directives de cette dernière. Son implication semble rester essentiellement passive. La seule proposition qu'il initie à l'égard d'Audrey se voit refusée. Audrey se sent-elle menacée dans son statut de meneur de jeu? On ne sait pas, car Stéphane n'insiste pas et retourne au statut qu'il occupait. Les différentes répliques de ces séquences sont initiées par Audrey, Stéphane n'en réalise que 7 sur 339. Stéphane ne marque pas d'opposition aux différentes initiatives, quelquefois il signifie son accord. Il accepte donc implicitement le statut, le projet global et les projets secondaires.

L'association des deux dyades apparaît ici satisfaisante aux membres de chaque dyade. Cependant, en poursuivant l'analyse des répliques dans les différents événements impliquant des interactions inter-dyade, celle-ci semble dégager une insatisfaction de la part d'Audrey. Une insatisfaction qui se témoigne d'abord dans l'initiation de plus de la moitié des événements; une insatisfaction qui se fait sentir dans la nature même des événements et due au fait qu'Audrey réagit vivement sur le plan personnel à l'ensemble de ces événements et peut-être, une insatisfaction sur le plan de la qualité de la relation entre Simon et Cédrik.

Les ambiguités émergent-elles de la qualité de la relation interpersonnelle entre les enfants? Ou simplement d'un désaccord sur le contenu? Cette question guide l'analyse des différents objets d'interaction pour chaque événement. 


\section{\begin{tabular}{|l|l|}
\hline Événement 1 & Statut des enfants \\
\hline
\end{tabular}}

Le premier événement survient lorsque Cédrik met en évidence le statut d'un membre de l'équipe: (28) "Attend c'est un petit bébé, c'est un petit bébé la la", et il s'attribue ce non-statut: (2) "Hein, chu pas un petit bébé", et le partenaire de Cédrik, Simon, embarque dans le même sillon en reprenant à son compte l'idée pour la diriger vers Audrey: (30)

“Toi t'en ai un, c'est rien qu'un petit bébé. Ah c'est laite ça. Hey, regarde!".

Mais avant que tout ceci prenne plus d'ampleur, Audrey réinvestit ce statut "personnel" sur le plan symbolique dans leur activité de construction: (31) "Nous autres, on a un petit bébé d'un an".

Ce premier événement rend compte du statut d'Audrey soit l'attribution du rôle de bébé par Cédrik et le partenaire de Cédrik, Simon, cautionne explicitement ce rôle.

Dans cet événement, Audrey, à notre sens, fait preuve de maturité et d'une bonne flexibilité en réinvestissant cette ambiguïté possible de la nature de la relation, soit du statut, sur le plan symbolique et en l'utilisant comme un rôle éventuel dans son activité de construction.

\section{\begin{tabular}{|l|l|}
\hline Événement 2 & Compétence de chacun \\
\hline
\end{tabular}}

Donc Audrey, en réinvestissant le statut de bébé, verbalise les intentions d'actions de son équipe: (32) "Aie, ben nous autres, aie, sais-tu qu'on la partie d'là, nous autres, là on va faire des chemins comme ça, on va rentrer dedans c'ta nous autres". Ainsi, elle relance l'autre équipe qui se repositionne dans ses intentions de projet: (33) "Ah, nous autres, on va rentrer dedans. Aie 42. Vous pourrez pas rentrer dedans mais nous autres on va pouvoir rentrer dedans. Hein Cédrik." Donc, il relance l'autre équipe sur les possibilités de leur projet tout en s'assurant de l'approbation de son partenaire. Audrey donne la réplique à cette relance mais cette fois-ci sur un autre qualificatif du projet: (36) "À va être belle". Audrey informe: "On va barrer, tu pourrais rentrer dans notre petite maison".

Et Simon avance une autre proposition: (39) "Oh nous autres est rien que des petits bonhommes de train" et Cédrik reprend cette idée et revient à l'idée de statut de bébé: “C'est rien 
que des petits bonhommes de bébé". Finalement, Audrey désamorce cette tangente par une stratégie de chantage affectif: (42) "Ben là, on sera pas ton ami, pis j'vas le défaire". Simon riposte et lui signifie de le laisser: "Oh ben, laisse-moi". "Y'a pas d'autres choses, nous autres, notre maison est plus grande, pis on fait pas du bruit, pis là on parle pu de ça". Par cette réplique, Simon met un terme à ce chantage et son partenaire recentre sur l'activité de construction en donnant une directive d'emplacement d'un bloc: (44) "Tiens, mets ça dans le coin". Ici, le fait de recentrer sur le contenu (projet global) fait tomber l'ambiguité quant au statut car cela met en danger le contenu de l'échange.

Dans l'événement 2 , il y a relance de l'ambiguïté relevée. À l'événement 1 , elle utilise une stratégie de chantage et de menace de défaire leur construction. À ce moment, en déplaçant l'ambiguïté de la relation sur l'activité de construction, c'est-à-dire sur le contenu, elle diminue ponctuellement l'amiguïté et elle recentre chacun sur son activité.

Ici, est-ce vraiment Audrey qui se sent menacée dans son statut de meneur de jeu ou l'ambiguïté serait-elle vécue dans la dynamique entre Cédrik et Simon et qu'Audrey masque par ses interventions?

À l'analyse des répliques de cette dyade, c'est Cédrik qui occupe le statut de meneur de jeu et Simon qui exécute dans l'ensemble de la séance les différentes directives de Cédrik à propos du transport du matériel et son agencement. Par contre, l'un et l'autre initient des propositions, des idées, et chacun semble partager le même niveau de communication car les répliques ne laissent pas transparaître d'ambiguïtés.

Est-ce que cette ambiguiité s'actualiserait dans les échanges d'événements à travers les négociations inter-dyade? Est-ce que Simon et Cédrik discutent leur statut de meneur de jeu ouvertement à travers leurs interactions dans les événements? Et quel rôle Audrey assure-t-elle dans cette dynamique?

\section{\begin{tabular}{|l|l|} 
Événement 3 & Infiltrations dans le sujet de l'autre dyade \\
\hline
\end{tabular}}

Interaction entre deux membres d'équipes différentes: Audrey et Simon. Audrey évalue les possibilités de rentrer dans la maison qu'elle construit. Cet énoncé suscite un questionnement de la part de Simon: "Vous rentrez pas dedans", ce qui amène Audrey à donner des informations et à argumenter: "Ben nous autres, moi pis Stéphane, on rentrera pas dedans 
parce que...". Simon spécifie que la construction est faite pour les deux: "C'est inc pour nous deux", donc il soude encore une fois son association avec Cédrik, ce qui encourage Audrey de nouveau à préciser sa propre association: (53) "Ben nous autres, moi pis Stéphane..." et (55) "Moi pis Stéphane" et à informer de ce qu'ils font. Cet énoncé suscite une relance de Simon: (56) "Oh ça va être super. Nous autres, nous autres, on est plus, plus succulents".

Dans cet événement, les interactions font apparaître un échange sur les compétences de chacun.

$$
\begin{array}{|l|l|}
\hline \text { Événement } 4 & \text { Interactions à propos du matériel et de sa répartition } \\
\hline
\end{array}
$$

Cédrik donne une directive à Simon: (68) "Prends-les toutes pour dans le coin" (afin de pouvoir ériger son mur). Simon répond en argumentant: (69). Audrey réagit en lui demandant de faire attention pour ne pas défaire leur construction (parce qu'elle a utilisé les mêmes blocs qu'ils veulent tous s'approprier pour ériger les murs de leurs constructions). Elle argumente également en soutenant: (71). Cédrik redemande de ne pas tous les prendre et qu'elle pourrait en laisser au moins un (72). L'interaction se termine de cette façon sans plus de discussion et Cédrik propose une idée de barrière à Simon (73) qui recentre l'activité de construction.

Ici, la stratégie de Cédrik quant à la possibilité de se répartir le matériel apaise l'ambiguïté sur le contenu. C'est encore en se recentrant sur la tâche par la proposition d'un projet secondaire de barrière que l'interaction prend fin. Cet événement fait ressortir une fois de plus les compétences de construction de chacun des enfants.

\begin{tabular}{|l|l|}
\hline Événement 5 & Limites de l'aire \\
\hline
\end{tabular}

C'est Cédrik qui amorce cette interaction: (78) "Aie, avait dit pas ça" (montre du doigt le territoire à respecter identifié par la chercheuse). (78) "Stéph! avait dit pas de passer ça". Mais Stéphane ignore.

Audrey reprend à son compte l'information de Cédrik: (79) "Dépasse pas" et Cédrik signale que: (80) "Stéph, lui, y dépasse par dehors". Alors Audrey canalise cette discussion en informant et recentrant sur l'activité en cours: et Simon fait de même, il informe et recentre sur l'activité de construction: (82). 
Et Simon et Cédrik continuent leur construction en donnant des directives sur la façon de placer les blocs et chacun accepte les propositions de l'autre. Ils démontrent une fois de plus leurs différentes compétences.

$$
\begin{array}{|l|l|}
\hline \text { Événement } 6 & \text { Observation / Compétence } \\
\hline
\end{array}
$$

Simon attire l'attention de l'autre équipe pour qu'ils observent comment ils font: (87) "Regardez comme nous autres, arrive à le faire". Par cet événement, Simon attire l'attention sur leurs compétences.

\section{\begin{tabular}{|l|l|l|}
\hline Événement 7 & Matériel et casier de rangement \\
\hline
\end{tabular}}

À propos du matériel et des casiers de rangement, Audrey intervient pour signifier la façon de placer le matériel dans le casier de rangement: (90) "Aie! Ça va même pas là, hein ça" (en montrant le rangement). "Ça va même pas là, ça va ici là". Mais Cédrik et Simon sont centrés sur leur activité de construction et sont à prendre une décision pour rapetisser une porte. Audrey attire l'attention de Simon et Cédrik sur leur non-capacité de rangement du matériel.

\section{\begin{tabular}{|l|l|}
\hline Événement 8 & Observation d'ingérence \\
\hline
\end{tabular}}

Audrey observe l'autre équipe et voit Simon qui a fait tomber un bloc en entrant dans la maison. Elle énonce un commentaire sans que personne ne lui demande son avis: (93) “Simon qui l'a défaite".

À ce moment, Stéphane passe à l'intérieur de la construction de Cédrik et Simon. Simon répond à Audrey (comme une façon de se défendre): (96) "On est capable de l'arranger", (97) "facile" et Audrey répond: (98) "Très facile", (99) “c'est facile arranger ça" (il rit) et Audrey dit: (100) "Par chance que j'ai pas défaite".

Simon et Cédrik continuent leur construction en proposant des idées: (104) "Laisse tomber ça" en enlevant les blocs du devant du mur; il les réutilise en les plaçant devant le balcon. Simon reprend la proposition de Cédrik: (107) "Laisse tomber ça" et ajoute que c'est facile à défaire (en enlevant les blocs du devant du mur et les réutilise en les plaçant de- 
vant le balcon). Audrey continue de construire et Stéphane place des cylindres aux quatre coins.

\begin{tabular}{|l|l|}
\hline Événement 9 & Observation / Évaluation de la construction \\
\hline
\end{tabular}

Audrey dit: (109) "Parce que nous autres là, on sera pas capables de le refaire" et s'adresse à Simon et à Cédrik. Simon intervient en donnant une appréciation sur la construction: (110) "Ah ben! c'est beau, vous pouvez traverser chez eux", propose deux idées: "Oh! Gardez ces étages" et "Aie! laisse-la d'même".

\begin{tabular}{|l|l|}
\hline Événement 10 & Commentaire et mise en garde \\
\hline
\end{tabular}

Audrey qui a observé que Simon passe dans sa maison pour aller au casier de rangement émet un commentaire: (114) "Tu passes par là". (115) "Parce que là tu défais toutes nos affaires pis on n'a même pas le temps de le défaire [refaire]". C'est Cédrik qui recentre à l'activité de construction: (117) "Vite, vite". (118) "On fait pas d'autres sortes", (119) ou encore, (120) "Aie! c'est..." et Simon (121) reprend la proposition de Cédrik.

\begin{tabular}{|l|l|}
\hline Événement 11 & À propos de la répartition du matériel \\
\hline
\end{tabular}

Audrey continue de construire mais: (122) et Cédrik répond à son intervention à propos du matériel: (123) "Aie, aie, vas-y n'emporte. Nous autres aussi on a besoin aussi nous autres, ça fait que toé tu as presque tout pris". (124) “[...] a mis là, là, là". (125) "T'es as presque tout pris". Et Cédrik dit: (126) "Ouais, toé pis ton Stéphane" et Simon renchérit: "Avec ton Stéphane là". Et finalement, Audrey clôture la discussion qu'elle avait amorcée en disant: (128) "Aie, tu veux-tu te fermer". (129) "Ouais, tu devrais ta fermer".

Ici, l'argumentation avancée semble se situer à propos de la répartition du matériel. Cependant, par la réplique de Cédrik: "Ouais, toé pis ton Stéphane", on glisse l'objet de la discussion au plan plus relationnel quant à la position d'Audrey par rapport à Stéphane. Cette réplique fait réagir vivement Audrey qui lui demande de la fermer.

La discussion à propos du matériel dégénère en attaque plus personnelle. Mais Audrey finit par recevoir des commentaires plus négatifs du fait qu'elle s'infiltre beau- 
coup et émet des commentaires de toutes sortes sur différents gestes et paroles de l'autre équipe. Cédrik recentre encore une fois cette interaction sur l'activité de construction.

\section{\begin{tabular}{|l|l|}
\hline Événement 12 & Directives sur les modalités de fonctionnement \\
\hline
\end{tabular}}

Simon avertit Stéphane de ne pas passer dans leur maison et Cédrik approuve l'avertissement de Simon et lui propose de faire le tour.

Audrey signifie: (146) "Nous autres, y'est très dur, pis on sera pu capables de le faire", mais cela s'arrête là car Audrey se recentre sur l'activité de construction. Encore la démonstration des compétences des enfants.

\begin{tabular}{|l|l|}
\hline Événement 13 & Enfant d'une autre aire \\
\hline
\end{tabular}

Jessie, un enfant d'une autre aire, entre au coin bloc. C'est Audrey qui lui adresse la parole: (203) "Jessie, Jessie! Est-ce que Michèle a dit que c'était bien?". Simon intervient: (207) "C'ta nous autres, ça" (il parle à Jessie parce qu'il embarque dans la construction; Simon le prend par le bras pour le sortir): "Ôte-toé d'là" et Cédrik approuve: (208) "Détruis-les pas". Audrey reprend à son compte la remarque de Cédrik (209) "Si tu détruis ceux-là, là, là, les affaires là, si tu détruis ça là là.". Simon s'engage dans une discussion avec Audrey à propos de la destruction et du droit qu'il a de faire ce qu'il veut: (210) "Ces affaires là, là, c'pas à toé, on a le droit de faire ce que l'on veut" et Audrey répond: (211) "Oui on a le droit" et elle poursuit en disant: (212) “On n'a pas le droit de défaire ça là là parce que, ben nous, nous autres, si on fait pas exprès, ouais, on n'a pas le droit" et Stéphane acquiesce les propos de Audrey: (213): "Nous, nous autres on a le droit de donner des coups de pieds" et Stéphane: (214) "Venez pas parce que..." (fait le geste de donner un coup de pied sur la construction de Cédrik et Simon).

C'est encore Cédrik qui recentre sur l'activité de construction en donnant une directive à Simon: (215) "Va me chercher des petits carreaux, va me chercher des petits carreaux de même" (en lui montrant ce qu'il veut). Cédrik est encore une fois mature et recentre son partenaire sur l'activité ce qui, à chaque fois, a été une stratégie qui a donné des résultats positifs. Et Simon se dirige au casier de rangement pour aller chercher le bloc demandé et lui apporte. II fait cela en chantant et transporte les blocs demandés. Cédrik chante et danse avec Simon en continuant leur construction. Cédrik remercie Simon d'avoir apporté les 
blocs demandés. Cet événement se concentre sur les droits et les responsabilités des propriétaires des productions.

\begin{tabular}{|l|l|}
\hline Événement 14 & À propos du matériel \\
\hline
\end{tabular}

Cédrik signifie leur intention de projet à Audrey et Stéphane: (226) "Nous autres, on fait une maison". Audrey constate qu'il n'y a plus de blocs dans le casier de rangement et met la responsabilité sur Simon et Cédrik. Simon constate qu'il n'y a plus de blocs et Cédrik constate qu'il en reste quatre et donne la directive à Simon d'aller chercher des triangles et Simon accepte. Ici, Cédrik recentre encore sur l'activité de construction en donnant une directive à Simon et Simon a utilisé tous les triangles disponibles, et Audrey s'excuse parce qu'elle prend les blocs par terre et les lance dans le fond du casier de rangement. Cédrik et Simon sont au casier de rangement pour apporter les blocs pour continuer leur plancher. Ils continuent de chanter en faisant leur construction. Cédrik réitère toujours ses directives pour Simon d'aller chercher des blocs.

Pendant ce temps, Audrey propose à Stéphane de se rattacher à la construction et ce dernier ne répond pas à cette proposition.

\begin{tabular}{|l|l|}
\hline Événement 15 & À propos du matériel accessoire \\
\hline
\end{tabular}

Simon reprend la directive à son compte et initie une interaction: (244) "Mais cher, nous autres on n'a pas besoin d'autos".

L'analyse de l'ensemble des événements met en lumière l'objet central des négociations, soit la compétence de chacun des membres dans l'activité de construction, les droits et les responsabilités en lien avec les concepteurs des productions. Les différentes répliques génèrent des discussions qui se centrent non pas sur le contenu de l'activité de construction elle-même mais sur le plan plus personnel qui implique la qualité de la relation interpersonnelle.

Ces différents événements permettent d'observer l'opérationnalisation de la dévalorisation et de la double injection, c'est-à-dire la reconnaissance de soi, comme compétent, et la reconnaissance de l'autre dans ses compétences. 
La stratégie de Cédrik indique cependant la recherche d'un moyen qui permet de diminuer ponctuellement l'ambiguïté au sein des statuts, soit de recentrer sur la tâche de construction en cours. En ce sens, Cédrik fait preuve de maturité et de compréhension de la situation. Il réutilise à chaque fois la même stratégie puisque celle-ci donne des résultats positifs. Cela empêche la dégénération de la situation et une rupture des interactions. Cependant, à chaque fois que la situation se représente, l'objet des négociations refait surface quant aux compétences de chacun.

Est-ce que ces enfants devraient trouver un terrain propice à l'affrontement et à la confrontation du vrai objet de négociation? Est-ce que Cédrik devrait user d'une autre stratégie pour permettre d'envisager l'objet répétitif et reproductif des négociations? Comme par exemple ne pas recentrer sur la tâche?

Un élément nous apparaît évident. L'analyse de ces différents éléments, soit la reproduction et la répétition de diverses situations qui produisent l'émergence du même objet de négociation, est un indicateur d'une problématique plus complexe que celle liée au contenu même de l'activité de construction.

\section{- $\quad$ Troisième phase: l'élaboration de l'activité de jeu symbolique}

La troisième phase, l'activité de jeu symbolique, se poursuit dans un mode simultané ou subordonné à l'activité de construction. Dans cette dernière, les enfants élaborent des projets globaux et secondaires qui, par la suite, donnent lieu à l'élaboration de scénarios dans l'activité de jeu symbolique. Rappelons que trois organisations dyadiques dans diverses séances observées présentent une activité de jeu symbolique simultanée sous différentes formes d'agencement à l'activité de construction: une simultanéité épisodique de courte durée de l'activité de construction dans l'activité de jeu symbolique, une simultanéité épisodique de courte durée de l'activité de jeu symbolique dans l'activité de construction et finalement, une simultanéité épisodique de longue durée de l'activité de jeu symbolique dans l'activité de construction.

Peu importe la forme d'agencement de l'activité de jeu symbolique à l'activité de construction, une chose demeure dans toutes les formes de participation sociale, c'est toujours la réalisation du projet global et des projets secondaires qui est le pivot de l'activité de jeu symbolique. 
Dans l'évolution du déroulement du jeu de l'enfant avec le matériel bloc, les interactions sociales générées dans la troisième phase ont comme but l'élaboration partagée de scénarios et comme objet de négociation les éléments de l'activité de jeu symbolique nécessaires à la création de scénarios élaborés par et autour des productions de l'activité de construction.

Dans cette troisième phase, les stratégies interactives déployées par les enfants sont les mêmes que lors du positionnement initial et de l'activité de construction, mais leur contenu se déplace sur les éléments du jeu symbolique qui rendent possible l'élaboration partagée du projet de jeu symbolique.

L'activité de jeu symbolique constitue la phase qui, dans l'ensemble des séances d'observation, suit celle de l'activité de construction. Pour illustrer le déroulement de l'activité de jeu symbolique et l'enchaînement des répliques traduisant les interactions sociales de cette activité, nous rapportons des extraits des neuf séances observées en dégageant le contenu des stratégies interactives des enfants afin de mettre en évidence, dans un premier temps, les éléments de l'objet de négociation dans cette troisième phase et, dans un deuxième temps, les éléments de l'objet de négociation dans les séquences présentant un désaccord intra-dyade et les événements de désaccord inter-dyade.

\section{- Les éléments de l'objet de négociation de la troisième phase}

L'extrait du deuxième épisode de la séance 4 est représentatif des diverses interactions générées dans l'activité de jeu symbolique. Examinons donc le contenu interactionnel de cette séance.

SÉANCE 4 ÉPISODE 2 BATEAU PIRATE (ENF3-ENF4)

Le projet global de construction de Jean-Benoît et Jonathan est la réalisation d'un bateau. Cette activité s'est poursuivie pendant 23 minutes. Par la suite, les deux enfants ont commencé à élaborer une activité de jeu symbolique. 


\section{JEAN-BENOÎT}

\section{4:49:27}

Aie! Salut pirate. (211)

24:55:06

Aie! Tu me connais? (213)

25:02:11

$O K$.

25:11:00

Ouais.

25:19:23

Awaye, regarde on attaque.

25:30:16

(Il lance un bloc et il me regarde)

$\mathrm{Ah}$ ! Sur mon bateau. (Se lève pour aller aider Jean-Michel)

\section{JONATHAN}

24:39:05

C'est inc pour jouer. (209)

24:40:00

Aie! Aie! Moé, c'est un bateau de pirates. $\forall$

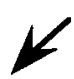

$\forall$

25:00:13

Aie! On habitait ensemble $O K$ dans ton bateau. Avant y faut que je lance. Aie! On faisait semblant qui était collé OK. C'était un gros bateau à nous toute seule.

\section{5:13:00}

Aie! Est-ce qu'on pourrait attaquer

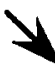
des bateaux de pirates? Un plus gros. (...)

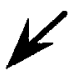

\ 25:22:00

Envoye! On peut attaquer le. Allez, allez, défends-toi, prends une bombe. Bravo!

\section{5:43:05}

Aie! Moi aussi je n'ai une manette. (Prend un bloc, le place debout à l'avant de son bateau)

Aie! (Change de voix) Aie! Jean-Benoît! Aie! Capitaine! Capitaine! Un bateau collé à côté de notre bateau. Capitaine! CA-PI-TAI-NE! Capitaine, je vous en prie répondez-moi (avec la main à la bouche) Capitaine! Re... Y'a un bateau de collé à côté de notre bateau. Venez vite. Est-ce que je lance une bombe? OK. À vos ordres capitaine. (222) 
26:18:24

Ouais (avec la main sur la bouche, en allant chercher une grande planche). (225)

27:15:20

(Il revient dans sa construction et s'assoit)

\section{7:53:02}

Moi aussi j'ai de l'huile.

\section{9:08:07}

Aie Jonathan! Amène-toi dans le bateau parce qu'on va à maison (imite le bruit du bateau en bougeant les bras et lance un autre bloc et imite le bruit du bateau) (246)

\section{6:38:29}

(Il se détourne et prend une bombe [bloc]). Je la lance juste sur le tapis. Ouah! C'est pas des vraies bombes, tu le sais bien (dépose et bouche les oreilles en imitant le bruit) $O h ! O h !$ C'est pas le bateau. Yep! Salut! Youp là! (229)

\section{6:59:23}

Ben là, ça va aller bien, notre bateau plein de dynamites à 40 moteurs, tu sais comment y'en a des moteurs? 40 moteurs. J'ai des moteurs, de l'huile, ça c'est de l'huile. (230)
29:13:26

OK. (248)

29:18:14

Non! J'ai pas le temps. Salut! Broum... broum... (imite le bruit du bateau). Bep! Bep!

29:29:01

Ah non! J'arrive. Ben si tu me le promets, $j$ 'ira, si tu veux que j'y vas, je vas te lancer une bombe. Ça va, ça va te ferait plaisir hein! Ça va te ferait plaisir hein! Aller une bombe. (Jean-Michel lance une bombe et il la reçoit sur les doigts). (251)

\section{9:47:00}

Aie...ye! Tu vois c'est quoi que ça fait. Je vas t'en lancer une bombe. Pouf? (lance un bloc). Aie! Plongé! Oh! Oh! Aie! Vite ton bateau y va couler, ton bateau (Jean-Michel lance un bloc sur le bateau de Jean-Benoît) Ton bateau, quoi, relance une bombe! (254) 
30:21:10

On en remette. (Prend un bloc, le met sur le côté du bateau pour remettre de l'essence). (260)

\section{0:58:23}

(Il prend un bloc et fait semblant de boire)
30:14:21

Non! Non! Je peux pas, je peux pu contrôler, y'a pu de gaz. (259)

\section{0:23:20}

Non! C'était ça le gaz! (en montrant un cylindre) C'est pas grave, on a trois bouteilles. (Fait semblant d'en boire) Plaq! Plaq! Dégueulasse. Aie! Ça c'était l'eau. Aie! Ça c'était l'eau OK. Eh! Moi j'ai soif, je commence à avoir soif. Aie! Le, la alors l'eau là, était là! $O K$. (Fait semblant de boire avec un bloc) Hum! Ça fait du bien, j'ai pas assez de boisson. Merci.

Dans cette activité de jeu symbolique, les interactions sociales relevées se regroupent sous cinq catégories différentes. Cependant, l'ensemble des types d'interaction présenté est la synthèse des neuf séances d'observation.

Une première catégorie d'interactions concerne les répliques assurant la transition entre la fin de l'activité de construction et le début de l'activité de jeu symbolique.

Dans plusieurs séances, les enfants annoncent explicitement cette transition. Les répliques suivantes traduisent la formulation de cette annonce: Bon là on commence à jouer. (SEA2-ENF); Aie! On commence à jouer. (43) (SEA1-ENF); On joue là, y fait toute ça, un là et un autre là, il m'en faut trois, m'en faut un autre, bon tu fermes la porte. (250) (SEA2-ENF1); C'est assez, va resserrer lui. Ça (en lui donnant un bloc qui reste). La maison est finie. (251) (SEA2-ENF4)

Une deuxième catégorie regroupe des interactions à propos d'éléments symboliques apparaissant dans l'activité de construction.

Ces éléments symboliques peuvent servir de prémisses à d'éventuels scénarios dans l'activité de jeu symbolique ultérieure pour:

- transformer symboliquement un bloc;

- partager cette nouvelle signification avec son partenaire; 
- proposer un rôle ponctuel:

Ex: Il y a des agents secrets dans notre château. (SEA3-ENF3) Un homme est mort. (SEA4-ENF4)

- signifier une connaissance à propos du rôle annoncé:

Ex: Il [agent secret] sait pas leur langue. (54) (SEA3-ENF3) Personne peut savoir leur langue. (55) (SEA3-ENF3)

- attribuer une signification à un bloc:

Ex: [...] Ah c'est ça le désert. (77) (SEA3-ENF3)

C'est les crocos, faut que tu regardes. (93) (SEA3-ENF3)

- des actions à partir de la signification:

Ex: Il faut traverser le désert. (80) (SEA3-ENF3)

Tu me lances des dynamites. (119) (SEA4-ENF3)

Je vais faire sauter ton bateau. (163) (SEA4-ENF3)

- proposer des thèmes éventuels:

Ex: On fait notre festival. (109) (SEA3-ENF3)

- réaliser une action isolée de faire semblant:

Ex: Bon je débarque du bateau et fait semblant de nager. Blop!

Blop! (236) (SEA3-ENF3)

On va aller se baigner l'autre bord de la terre. (265) (SEA3-ENF3)

- le choix d'un matériel accessoire et de certaines onomatopées:

Ex: VRR!..... VRR! (en faisant rouler la voiture)

- attribuer à l'autre un rôle ponctuel:

Ex: Aie! Capitaine, y nous reste plus de bâtons. (322) (SEA3-ENF3)

Capitaine, y nous reste plus de grandes planches. (323) (SEA3-ENF3)

- s'attribuer à soi-même un rôle ponctuel:

Ex: Là, là, je vais m'appeler PENTA. (113) (SEA4-ENF4).

Moning! Moning! Attaquez les autres tout de suite. (SEA4-ENF4)

- des actions ponctuelles possibles:

Ex: Attends-moi, attends-moi! Je vais faire exploser le village de... (182) (SEA4-ENF4)

La troisième catégorie d'interactions sont à propos des éléments nécessaires à la mise en place du thème pour:

- proposer un thème;

- proposer à l'autre une éventuelle action possible dans un éventuel scénario:

Oh! On va aller se coucher. (SEA2-ENF)

Là tu vas avoir à aller dans le château de la sirène.

Là, la sirène a viendrait se baigner avec son chef.

Là, il laisserait dans l'eau, il laisserait dans l'eau pis la sirène [...]

On va aller se baigner dans la piscine du géant.

- proposer des rôles possibles;

- définir un contexte à partir de la production réalisée dans l'activité de construction:

Ex: Mon auto, on va embarquer à quatre dedans. (396) (SEA3-ENF3) 
- recadrer le contexte physique:

Ex: Non, c'est une vitre d'auto, on est dans une auto. (409) (SEA3-ENF3)

- attribuer des significations à des blocs:

Ex: Aie! Mon envoyeur de fumée, VROUM... VROUM... VROUM... (401) (SEA3-ENF4)

- annoncer le début du scénario:

Ex: Cédrik, on est prêt à partir en auto (Cédrik et lui se sont assis dans l'auto. Il a pris un double module pour simuler le volant). BROUM... BROUM! (SEA3-ENF4)

Une quatrième catégorie fait ressortir les interactions à propos des éléments à l'intérieur du scénario, soit des éléments constitutifs au développement du scénario pour:

- établir le contexte physique du scénario:

Ex: C'est un beau bateau de pirates. (210) (SEA4-ENF4)

- attribuer des significations particulières à des parties de la construction:

Ex: Ça, c'est notre lit.

Ça, c'est une balançoire.

- expliquer;

- clarifier;

- verbaliser son action en cours dans le scénario:

Ex: Ah là là! Je vais à la cuisine.

Lui s'en va dans l'auto de la sirène, $\mathrm{y}$ va, $\mathrm{y}$ va dans sirène.

Bon je starte mon auto. (452) (SEA3-ENF4)

Je vas exploser un peu de poisson. (184) (SEA4-ENF4)

Je vas plonger dans l'eau. (185) (SEA4-ENF4)

- proposer des actions à un personnage dans le vif du scénario:

Ex: Tu vas le mettre par terre. On va le mettre là.

- proposer des actions aux choses du scénario:

Ex: Y'a des bombes qui éclatent. (251) (SEA4-ENF4)

- spécifier des découpages temporels:

Ex: C'est la nuit.

- distribuer des tâches spécifiques:

Ex: Aie! On prend pas des dynamites dans mon bateau. Allez! Va-t-en! (334) (SEA4-ENF4)

- distribuer les espaces:

Ex: C'est moi qui est en avant, c'est moi qui est là. (433) (SEA3-ENF4)

- inviter l'autre dans son action:

Ex: Viens dans l'auto. (440) (SEA3-ENF4)

- imiter le bruit:

du bateau

de l'auto

de la sirène

de l'explosion 
- attribuer à l'autre un rôle:

Ex: Aie! Salut pirate. (211) (SEA4-ENF3)

- s'attribuer à soi-même un rôle;

- questionner sur des actions possibles:

Ex: Aie! Est-ce qu'on pourrait attaquer des bateaux de pirates? (216) (SEA4ENF4)

Une cinquième et dernière catégorie présente des interactions à propos de la métacommunication:

Ex.: C'est inc pour jouer. (209) (SEA4-ENF4)

Aie! On habitait ensemble, OK, dans ton bateau. Avant, y faut que je lance. Aie! On faisait semblant qui était collé, OK. C'était un gros bateau à nous tout seul. (213) (SEA4-ENF4)

Ouah! C'est pas de vraies bombes, tu le sais bien. (SEA4-ENF4)

Ces interactions sociales ne font pas état de toutes les interactions générées par l'activité de jeu symbolique à deux, trois ou quatre, mais elles sont représentatives des interactions impliquées dans la mise en place et le déroulement des éléments du jeu symbolique des divers scénarios élaborés.

L'ensemble de ces interactions dans l'activité de jeu symbolique met en évidence des habiletés, des compétences et des stratégies souvent très élaborées et complexes qui contribuent à un enchaînement cohérent et de plus en plus productif dans le dénouement de l'activité de jeu symbolique. Ces interactions sont des indicateurs d'une bonne maturité et d'une bonne décentration de son point de vue. Les uns les autres proposent, ajoutent des spécificités au projet de l'autre, reprennent des idées à leur compte, ajoutent, modifient, contre-proposent, améliorent, acceptent, refusent mais toujours dans le respect de l'autre, de ses idées et de ses compétences. L'intérêt et le souci de "rester ensemble" semblent être le point central des différentes stratégies négociatives: trouver un terrain propice à l'expression et à l'élaboration de scénario.

- Séquence de désaccord intra-dyade

D'une manière favorable, certaines interactions provoquent une confrontation entre les enfants afin de les obliger à faire face à une situation problématique. C'est le cas des séquences intra-dyade où deux enfants arrivent difficilement à un dénouement satisfaisant de la situation. Les interactions présentent à la fois des stratégies de résolution de 
problèmes qui échouent du fait que des phases du processus de résolution de problèmes ont été escamotées.

Est-ce que les interactions des enfants les amènent à bien identifier la nature de leur problème? Quelles sont donc leurs stratégies d'identification? Quelles sont les stratégies d'élucidation et les stratégies de résolution?

Un extrait de la séance 6 permet d'apprécier l'enchaînement et le dénouement d'une séquence de désaccord intra-dyade.

Au début de la séance, pendant les cinq premières minutes, les tentatives d'association se soldent par quatre projets individuels de l'activité de construction. Deux enfants, Jean-Benoît et Elizabeth, ont initié des demandes d'association, mais en vain. Donc, chacun s'affaire à un projet individuel de construction. Cependant, 23 événements se produisent tout au long de la séance de jeu. Ils traduisent l'intérêt que chacun porte à l'autre mutuellement pour le partage de l'espace de l'aire, pour le partage du matériel, pour la production de l'autre, pour des informations sur les projets, etc.

Voici la liste de ces interactions dans les événements:

\begin{tabular}{|c|c|c|}
\hline Événements & Objet de l'interaction & Initiateur de l'événement \\
\hline $1^{\mathrm{er}}$ & Attire l'attention sur son projet & Stéphane \\
\hline $2^{e}$ & $\begin{array}{l}\text { Attire l'attention d'un enfant d'une autre aire sur son } \\
\text { projet }\end{array}$ & Jean-François \\
\hline $3^{e}$ & Interpelle & Elizabeth \\
\hline $4^{e}$ & Veut prendre part au projet de l'autre & Jean-François \\
\hline $5^{\mathrm{e}}$ & Hors aire, évaluation de son projet & Stéphane \\
\hline $6^{e}$ & $\begin{array}{l}\text { A propos du matériel accessoire (veut avoir la voiture de } \\
\text { l'autre) }\end{array}$ & Jean-François \\
\hline $7^{\mathrm{e}}$ & À propos du matériel (revendique son bloc) & Stéphane \\
\hline $8^{e}$ & À propos d'une fête & Elizabeth \\
\hline $9 e$ & $\begin{array}{l}\text { Lance du matériel accessoire sur la construction de } \\
\text { l'autre }\end{array}$ & Jean-François \\
\hline $10^{e}$ & Attire l'attention sur son projet & Stéphane \\
\hline
\end{tabular}




\begin{tabular}{|c|l|c|}
\hline Événements & \multicolumn{1}{|c|}{ Objet de l'interaction } & Initiateur de l'événement \\
\hline $11^{\mathrm{e}}$ & Offre de l'aide & Jean-Benoît \\
\hline $12^{\mathrm{e}}$ & Embarque dans la construction de l'autre & Jean-François \\
\hline $13^{\mathrm{e}}$ & Donne des informations sur sa production & Stéphane \\
\hline $14^{\mathrm{e}}$ & Veut prendre le bloc de l'autre & Stéphane \\
\hline $15^{\mathrm{e}}$ & Attire l'attention sur son action & Jean-Benoît \\
\hline $16^{\mathrm{e}}$ & Propose une action de faire semblant & Jean-François \\
\hline $17^{\mathrm{e}}$ & Donne du matériel accessoire autobus & Elizabeth \\
\hline $18^{\mathrm{e}}$ & Propose une action & Elizabeth \\
\hline $19^{\mathrm{e}}$ & Informations & Stéphane \\
\hline $20^{\mathrm{e}}$ & Veut participer au jeu des deux autres & Jean-Benô̂t \\
\hline $21^{\mathrm{e}}$ & Lui donne son idée (quoi faire) & Jean-Benô̂t \\
\hline $22^{\mathrm{e}}$ & Propose aux deux autres une destination, une idée & Jean-Benô̂t \\
\hline $23^{\mathrm{e}}$ & Revendique les droits par rapport au bloc & Jean-Benô̂t \\
\hline
\end{tabular}

Cependant, après cinq minutes de l'activité de construction, Jean-François annonce: "Moé chu prêt", ce qui suppose dans le contexte qu'il est prêt à commencer à jouer et effectivement il débute seul une activité de jeu symbolique. Il amorce donc un long monologue sur le déplacement de ses voitures sur son autoroute, monologue qui se poursuit pendant 12 minutes. À ce moment, il annonce qu'il va faire une ferme et prend différents animaux dans le bassin des animaux et amorce un autre monologue, cette fois-ci à propos des différents animaux qui sont dans sa ferme.

Après 22 minutes de jeu solitaire, on voit apparaître les prémisses d'une possible association par l'intermédiaire du personnage singe, qui tente d'entrer dans l'édifice de Stéphane (il est à ce moment dans la construction de Stéphane qui tente de lui fermer les blocs pour ne pas que le singe entre). Voici l'extrait de cette séquence.

Jean-François “'...] (Il prend le singe) Je veux rentrer dans, bon (le singe qui parle). Oh j'ai peur! Ben moi, je veux rentrer dedans, bon! (il est dans la construction de Stéphane avec son singe). Non, là, là, y'a un bout! Je veux rentrer, tu m'as coincé le nez, tu m'as coincé le nez, je peux pas rentrer là, c'est plus creux (en poussant la planche). Non, peux pas rentrer (parce que Stéphane referme la planche). Je vas la débâtir."

Stéphane “Non Jean-François!” (parce qu'il est en train de faire tomber son bloc de chaussée) 


\begin{abstract}
Stéphane "Aie, aie, aie! Arrête de niaiser!"
Jean-François (23:33:20) (déplace le singe partout sur l'édifice et imite le bruit du singe. Il arrive au-dessus de l'édifice devant le miroir) "Aie! Je me vois!" (fait parler le singe)

(23:41:12) "Ôte-toi, tu vas démolir, tu vas faire tout casser là, tu vas faire tout casser là" (Stéphane met ses voitures dans le garage et referme son toit [planche de grande construction])
\end{abstract}

Jean-François retourne avec son singe à sa construction. Il utilise l'incarnation du personnage singe pour tenter d'entrer dans la construction de Stéphane. C'est par le personnage que passe le message de son intention, alors que Stéphane, lui, s'adresse directement à Jean-François et n'entre pas dans la stratégie du personnage. Stéphane manifeste très explicitement son refus à Jean-François lui-même, ce qui permet probablement à Jean-François, par l'intermédiaire du rôle du singe, de continuer à grimper sur l'édifice de Stéphane malgré son refus. Stéphane revient à la charge très clairement. Finalement, Jean-François, par l'intermédiaire du singe, retourne à sa construction.

Ici, l'échange se passe à deux niveaux: un des partenaires échange sur le contenu du jeu de fiction et l'autre partenaire échange sur la personne elle-même. Il n'y a ni accord sur le plan de la fiction dans la reconnaissance du rôle du singe, ni accord sur l'acceptation de Jean-François et de son rôle (lui-même) dans cet épisode de fiction.

Après ces échanges, Jean-François cherche ce qu'il va faire. Il veut aller dans un autre atelier mais il connaît la consigne pour ceux et celles qui sont au coin bloc. Finalement, la chercheuse intervient: "Je suis sûre, si tu pensais bien dans ta tête, t'es capable de continuer. Je suis sûre de ça."

Finalement, Jean-François revient à la charge (27:30:00) auprès de Stéphane et se positionne cette fois-ci de personne à personne pour lui faire une proposition d'association: “Aie Stéphane! Ça là (en utilisant le quadrangle qu'il a toujours gardé dans ses mains), ça pourrait être pour ce que je peuve monter dans ton édifice (en plaçant le quadrangle en pente à son édifice et à l'autoroute). Comme ça là je pourrais grimper jusqu'au bout. Scuze-moi! Excuse-moi! (passe par dessus Stéphane et va chercher un module, le replace dans le casier). J'ai une idée, une très bonne idée (prend un quadrangle) très très bonne idée d'ailleurs." 
À ce moment-là, Stéphane lui répond: "Tu peux rentrer" (en soulevant le module du dernier étage).

\author{
Jean-François "Ah! Pas tout de suite, j'ai pas fini." \\ Jean-François "Bon (prend un module), qu'est-ce que pourrais aller faire? Je vais aller faire ça de \\ même, un autre (module)."
}

Est-ce qu'il veut garder un statut de meneur et de décideur?

Stéphane lui offre de l'aide: "Moi je vas le tenir" et tout de suite Jean-François répond: (il place un demi-module).

Donc, comme c'est lui qui a proposé l'idée, c'est lui qui SAIT. Alors Jean-François se place bien dans une position où il reconnaît et souhaite sa collaboration, mais une fois cette position acceptée, il veut aussi bien faire passer son double message: il veut bien collaborer ensemble mais c'est lui qui sait le mieux comment faire. En indiquant la suite du jeu, Jean-François peut ainsi se présenter comme celui qui peut en restructurer le déroulement.

Jean-François "Tiens! Là je peux monter dans, au bout de ton édifice hein! Je te l'avais dit hein! Bon je m'en vas dans l'édifice au bout haut, haut (en faisant rouler sa voiture). Avais là, je peux y aller. Ah ça l'air épeurant! Arrête don!" (à Stéphane qui veut enlever l'auto).

Alors Stéphane propose: "Aie! Attends un peu! On va serrer les autres." "Non, non, non, non, non, non, t'as pas le droit."

La réponse de Jean-François à l'idée de Stéphane vient consolider sa position de meneur de jeu. Alors, Stéphane utilise une stratégie pour lui-même: "Je vas aller chercher mes autos" (il enlève le quadrangle, la planche de grande construction et va chercher ses autos). Ainsi, en adoptant cette stratégie, il ne menace pas le terrain de Jean-François. "Ousse qui sont? Là dedans?" (tient la planche de grande construction).

Ainsi, par cette réplique, Jean-François reconnaît les compétences de Stéphane qui a le droit de décider pour lui. Alors, Stéphane dit: "Non, sont ici les deux qui manquent. Ah ben là! Je vas faire une autre bâtisse, moé, moi je fais l'escalier." Ainsi, Stéphane 
aurait retrouvé sa pleine confiance en lui et son pouvoir de décision et de proposition, donc il est prêt à relancer le jeu.

Mais Jean-François: "Laisse là là, moi, moi, je fais les escaliers. On va la placer de même. on va la mettre de même. Ça serait-tu mieux une planche? Ça où est les planches? (soulève la planche de grande construction). Sont dans, sont dans..."

Immédiatement, Jean-François renvoie un message à double sens: je reconnais tes compétences de décision, pour toi, mais en même temps, il semble suggérer que c'est lui qui restructure la suite du déroulement de jeu et c'est lui qui sait comment les placer.

Alors Stéphane répond: "On va le placer de même, c'est moi qui la refait parce que je l'ai faite tomber". Donc peut-être une certaine stratégie de concession de Stéphane et un certain droit de refaire parce que c'est lui qui l'a fait tomber.

Jean-François: "Comme ça (en replaçant le bas de la pente). Non c'est moi! Ça va de même." Est-ce que dans cette réplique, Jean-François résume la confusion sur les compétences de Stéphane?

Stéphane: “Ça ah! (en replaçant la chaussée et fait rouler son auto) Jean-François signifie qu'ils ont tout fini ce qu'il fallait faire et il grimpe avec sa voiture sur l'édifice: "Ben moi, je grimpe."

Stéphane fait de même: il fait rouler sa voiture sur le carrefour et dans l'espace, puis dans la descente et sur l'autoroute, remonte dans le miroir et installe la voiture dans la chaîne de la toile persienne. À ce moment, sur le plan symbolique, il dit: "Au secours! Chu pris dans un piège" (se sort lui-même du piège et roule dans la descente).

Alors, Jean-François poursuit sur le plan symbolique différents gestes et paroles, ce qui peut laisser supposer qu'il reconnaît et accepte les compétences de Stéphane sur le plan symbolique et ainsi poursuit avec lui, sur le même plan sans ambiguitté. Ils continuent leur dialogue et s'enchaîne une explosion de l'édifice et l'importance se sortir de là et de s'en retourner chez eux et d'ouvrir une porte secrète. Il accepte que Stéphane ouvre la porte secrète, il y a aussi un passage et là, dans une réplique, Jean-François dit: "que là c'est à lui, c'est ta maison et pis là c'est sa maison". Stéphane refuse et dit: "qu'ils ont 
chacun un garage" et Jean-François accepte et continue sur le plan symbolique et se demande s'il y a assez de place dans le garage pour toutes ses voitures. À ce moment (35:36:21), Stéphane propose de mettre les autos ensemble et une obstination s'amorce entre les deux enfants, une série de huit oui/non et un "Non, je veux pas, je veux pas mêler les autos".

Ici, quelle ambiguïté cette proposition de Stéphane met-elle en évidence? (Tout semblait bien se dérouler.) Est-ce que dans cette proposition, Jean-François reconnaît que Stéphane veut prendre le statut de meneur de jeu? Et sur le plan de la réalité, il ne lui reconnaît pas les compétences de décideur lorsque la décision concerne la structure du déroulement du jeu. Alors Stéphane réagit vivement en disant "Oui! Oui! Regarde!" (prend ses voitures dans l'étage et les sort, fait tomber ses voitures du haut de l'étage). Donc, une stratégie d'affirmation, et Jean-François réagit aussi vivement: "Non! Là mon passage se ferme! Là mon garage se ferme! Mon garage est fermé. Mon garage est fermé. Mon garage est fermé."

Cette réplique par l'intermédiaire du plan symbolique implique-t-elle aussi toute fermeture à la proposition de Stéphane? L'association est menacée! Le conflit semble s'accentuer. Alors une troisième personne intervient, Elizabeth: "Y peut aller le rejoindre lui." Cette proposition d'Elizabeth signifie-t-elle: oui tout est fermé? Donc Stéphane ne peut entrer, mais lui, il peut aller le rejoindre. Elle lui propose une alternative. Est-ce que dans cette proposition, elle laisse supposer qu'il aille rejoindre Stéphane sur le plan symbolique? Extrapolons-nous trop?

Et finalement, Jean-François monologue: "Arrêtez à côté! Ah oui! Moi chu dans mon garage. Ouais, c'est le fun hein dans notre garage! Ah oui! Ben moi, j'ouvre ma porte de garage (fait le bruit de l'ouvrir). Ouvre-la pas trop grande hein! Non! Non! Bon là assez ouverte. Oui ...ui. Bon là là j'étais là mais moi je recule hein! Je pense euh! euh. Pouk Ben oui, c'est ça, je vas reculer là mais moi là faut vite que j'aille là (rentre la voiture dans le garage) pour pas que les voleurs me pognent (ferme la porte du garage). Tiens, j'ai fermé la porte!" "Moi j'ai soif" (il sort de l'aire).

Beaucoup d'interprétations peuvent se présenter. Jean-François a-t-il rejoint Stéphane sur le plan symbolique et a-t-il réouvert la possibilité de jouer ensemble? L'ouverture de la porte du garage est-elle l'ouverture au jeu? Tout cela n'est pas évident 
mais une chose est certaine, il manifeste un intérêt pour continuer de jouer avec Stéphane en parlant de "notre garage" et en relançant de lui-même le jeu, puisque c'est lui qui y avait mis fin. En allant prendre de l'eau, il va également prendre un peu d'air et de recul face à la situation, comme pour réaliser une rupture ponctuelle de la situation conflictuelle. Sortir de l'aire, pour prendre de l'eau tout de suite après la fermeture du jeu, aurait pris une toute autre signification. Lorsqu'il revient dans l'aire, il fait le constat que tout est débâti et qu'il faut qu'il recommence ce qu'il avait à faire.

Est-ce que la responsabilité liée au statut de meneur de jeu et de décideur l'est également dans la responsabilité de tenter les premiers pas d'une réconciliation, d'un certain équilibre pour que le jeu se poursuive?

Alors, Jean-François repositionne le jeu sur le plan de la réalité, pour faire la distribution des garages: "Aie, ça c'est mon garage! Ça c'est mon garage! Ces ceux garageslà sont à moi. Les deux garages-là sont à moi!" Et hop là, c'est reparti. Stéphane: "Ah non!"

Est-ce que cette réponse ne remet pas le conflit sur le plan de qui décide, qui conduit le jeu? "Regarde! Attends un petit peu! Moi j'ai deux garages, toi t'as trois garages. Toi, t'as ces trois-là, moi j'ai ces deux-là. OK."

Nous pensons ici que la réplique de Jean-François laisse supposer qu'il connaît certains éléments de la négociation, car il sait que l'édifice est à Stéphane et que s'il utilise la stratégie d'en donner plus à Stéphane, il lui reconnaît ses droits de propriété. Donc une stratégie qui lui permet toutefois de demeurer le décideur. Mais Stéphane refuse: "Non, je t'ai joué un tour pendant que t'étais parti. Chu t'allé les mettre."

Ici, il est difficile de comprendre cette stratégie. Est-ce que Stéphane voulait luimême décider de ses garages? Difficile à dire et de faire ce choix en l'absence de JeanFrançois et de le placer devant le choix accompli. Jean-François reprend "Aie! Pas là, $c$ 'est ton garage tandis que moi j'ai moins d'autos, $j$ 'aurai quatre garages, cinq avec lui peut-être". La réplique de Jean-François confirme notre hypothèse du choix de Stéphane de son garage. Alors, Stéphane dit: "Non, j'en prends juste un moi, ça va être lui" (le sixième). Ici, la réplique de Stéphane peut supposer que le nombre de garages lui importe 
peu. Il en veut seulement un mais qu'il veut le choisir lui-même et non qu'il lui soit attribué par Jean-François.

Alors, Jean-François dit: "Moi je prends les trois autres, OK." Donc, comme il laisse Stéphane choisir son garage, lui aussi peut à ce moment-là, choisir ses garages.

Stéphane: "Non, on s'en choisit un seulement." La réplique de Stéphane ramène la discussion sur le plan de la relation. Qui est le décideur? Qui prend les décisions? Qui mène le jeu? Qui choisit?

Et là, Jean-François se rallie sans discussion et sans stratégie, car il peut supposer la fragilité de leur association. Il ne veut pas mettre en cause cette possibilité de jouer avec Stéphane.

Cependant, un troisième enfant le confirme dans son choix car il dit: "Ben moi, j'aimerais ça n'en choisir un!' Alors, par sa réplique, Jean-Benoît remet une partie du problème au grand jour, c'est-à-dire, probablement la chance qu'il possède, car lui au moins a la possibilité d'en choisir un. Peut-être aussi que Jean-Benoît aimerait bien qu'on l'invite à s'associer avec eux pour jouer!

Jean-François ne s'avoue pas tout de suite vaincu et revient à la charge: “Est-ce qu'on peut n'en choisir deux? Deux chacun?" Par sa réplique, il y a une demande sousentendue de permission ou du moins, il induit la possibilité de choix, chose qui dans ses autres répliques, n'était pas permise. Il se positionnait comme le décideur des garages.

À ce moment, Stéphane profite de cette réplique pour reprendre son statut de décideur, de meneur de jeu et dit: "Lui, pui lui" (en désignant le sixième et le cinquième). Donc, il répond positivement à une partie de la demande de Jean-François, c'est-à-dire à la partie du choix possible de deux garages, mais ne laisse pas la possibilité à JeanFrançois de choisir ceux qu'il veut. Et là, il prend l'initiative de lui désigner lesquels.

Alors, il y a reprise du désaccord: Jean-François "Non! Moi je prends ces deux, lui pis lui. Toi tu vas en avoir trois, lui, pis lui, pis lui."

Ici, Jean-François reprend la position de décideur et du nombre et de la désignation. Toutes les stratégies déployées jusqu'à maintenant dans la compréhension de la dynamique semblent revenir à la case départ. 
Jean-François dit: "Non, on en prend deux chacun." Alors Stéphane revient avec l'avant-dernière réplique de Jean-François quant à la possibilité de choisir deux garages chacun. Devant la réplique de Stéphane qui lui fait voir l'évidence de sa propre proposition, Jean-François se restreint à choisir deux garages mais seulement les siens: "Oh moi, je prends ces deux-là!". Alors Stéphane lui signifie son choix et le choix de JeanFrançois: "Moi je prends lui, pis lui (le 5e et le 6e), toi tu prends lui, pis lui." (1).

Il prend donc les deux garages qu'il avait d'abord désignés à Jean-François mais est-ce que sa réplique du cinquième et du sixième garages voulait signifier une désignation pour Jean-François ou son propre choix? Parce qu'ici, Stéphane revient au cinquième et sixième garages ce qui laisse supposer que c'était peut-être son premier choix.

Là-dessus, Jean-François concède et propose de continuer avec les autos: " $O K$ là, je sors mes autos de d'là, toi aussi" (1). Donc, ici, Jean-François recentre la discussion et l'échange vers une possibilité de jouer avec les autos et en profite pour dire aussi à Stéphane qu'il devrait aussi le faire.

Veut-il par là prendre un statut de restructuration du jeu? Toujours est-il que Stéphane accepte cette proposition et ils s'entendent mutuellement pour enlever "plein de stock", comme dit Stéphane.

Jean-François "Ouais! Là, parce que là, y faut enlever mes autos, pis y faut enlever la grande planche. Ça c'est à moi (l'auto).

Ça c'est à moi.

Ça c'est à moi.

Ça c'est à moi.

Ça c'est à moi.

Ça c'est à moi.

On peut remettre la grande planche."

Alors, Stéphane demande: “Aie, on peut-tu n'en rajouter trois?” et Jean-François répond négativement à cette proposition: "Ah non. OK. Bon, ben là je mets mes autos dans un garage pis, pis 1-2-3-4..." Il répartit ses voitures également dans ses garages et ils s'entendent, en principe, sur la suite de la répartition des voitures. Mais pendant très peu de temps, car Jean-François revient à nouveau à la charge: “ $T$ 'as pas le droit, hein, de venir dans ces deux-là, c'est à moi". Ici, par la réplique de Jean-François, il remet en cause les droits et les propriétés de chacun, donc des éléments qui demeurent encore am- 
bigus. Est-ce les droits et propriétés de chacun qui sont discutés, ici, ou sont-ils un prétexte pour camoufler le vrai sens de cette ambiguïté liée à la nature de la relation interpersonnelle, et non à ces droits et propriétés qui semblent liés au contenu du jeu? Où se situe vraiment l'ambiguîté?

Par la suite, Jean-François reprend en lui redonnant un certain pouvoir sur son propre choix: "Moi, j'en mets, toi comment t'as d'autos?". Stéphane répond: "Trois". Jean-François: "Trois, moi j'en ai dix alors j'en mets trois dans chaque garage". Et JeanBenoît vient en tierce personne pour essayer de dresser le tableau de la situation: "Non, je le sais, c'est une dans chaque garage" (à Stéphane et Jean-François).

Ici, comme Jean-François a dix autos et trois garages, il doit en mettre trois dans chaque garage. Si Stéphane a trois autos et trois garages, il devrait en mettre une, dans chaque garage. Jean-François répond: "Non, y peut pas! Ah ouais! Une dans chaque garage".

Comme s'il n'avait pas compris au début la logique de Jean-François, Stéphane répond: "J'en mets trois! Non, regarde, j'en ai d'autres garages!" Et voilà, le débat est relancé sur la répartition des garages. Mais est-ce vraiment le vrai objet de l'ambiguiité? Si la situation était claire, cette ambiguïté surgirait-elle à nouveau? Jean-François continue: "Aie! Attends un petit peu! Attends un petit peu! Attends un petit peu! Moi, je prends les trois autres, OK? On prend chacun trois."

Stéphane répond: "Aie, dis pas que ça c'est à moi, je l'ai jeté dans le panier" (dans le bassin d'autos). Jean-François: "Moi je vais mettre deux dans chaque. Aie! Y'en manque une! C'est elle. Veux-tu que je t'en donne une? À part ça, ça marche pas" (lance sa voiture dans le bassin).

Dans cette réplique, on remarque que Jean-François reproduit des stratégies dans son répertoire de stratégies connues, ici en donnant l'illusion qu'il peut lui en donner une, donc qu'il fait un pas vers des négociations plus fructueuses. Mais immédiatement il revient sur ses positions en disant: "À part ça, ça ne marche pas". A-t-il anticipé qu'ils sont en train de revenir sur le même terrain conflictuel de message à double sens? 
Jean-François poursuit: "Aie, ousse qui sont mes autos? Moi y m'en manque deux des autos, OK?. Ah ouais." Stéphane dit: "Ça c'est à moi". Soupçonne-t-il Stéphane d'avoir pris de ses autos, sans sa permission? Et Jean-François "Ah c'est vrai, y va là!". Peut-être revient-il sur les suppositions qu'il a faites, de peur de briser l'association et de ne pas pouvoir trouver un terrain propice à la négociation? C'est comme si cette réplique annulait les suppositions. Ou s'est-il tout simplement trompé et ce sont les autos de Stéphane?

$\begin{array}{ll}\text { Jean-François } & \text { "Ben là, ferme les portes" (met les doubles modules portes) } \\ \text { Stéphane } & \text { "Ben là, tu l'installes de même" (en plaçant le double module) } \\ \text { Jean-François } & \text { "Non, moi c'est pas ce que je veux!"” }\end{array}$

Donc, il est bien difficile de s'entendre sur ce que chacun veut et la façon de concilier les vouloirs de chacun dans cette situation ludique. Est-ce la signification du statut de chacun qui revient à la surface? Qui conduit? Qui décide?

Et voilà que Stéphane dit: "Aie! C'est moi qui l'a fait l'édifice!" Par cette réplique, Stéphane ne cherche-t-il pas explicitement le motif ou la base de toute l'ambiguitté créée dans cette situation? Si c'est lui qui a fait l'édifice, est-ce qu'en même temps cela lui donne le droit de la responsabilité de décideur et de son vouloir à lui? Comme il est le concepteur et le propriétaire de la production, est-ce que cela lui accorde automatiquement certains droits ou tous les droits du statut de meneur et de structureur du déroulement des événements, des actions, de la structuration qui découlent de sa production? Cela confinet-il automatiquement, le rôle de l'autre dans un rôle ou un statut d'exécuteur, ou de celui qui applique les décisions de l'autre et qu'il doit jouer dans le sens que le meneur décide? Sa réplique abonde-t-elle dans ce sens? Du moins, une base différente et explicite semble posée.

Est-ce cette face cachée du message que Jean-François n'accepte pas? Est-ce que c'est ce rôle sur le plan de la relation interpersonnelle? Jean-François répond: "Regarde, ce que ça fait, c'est tannant! Moi, est-ce que je peux en mettre un de même? Ben l'à, y m'en manque un là. Regarde là. On voit encore des autos. Comment tu fais pour y aller?"

Quand il dit "Regarde ce que ça fait, c'est tannant", on peut penser, selon les répliques antérieures, qu'il interroge l'installation du double module proposée par Stéphane. Mais soulève-t-il également le fait que cela aussi est tannant? Les enjeux sont ouverts. 
Jean-François: "C'est qui l'autre? Ah y'est là" (en parlant de la voiture). Et Stéphane répond: "Dis là là, y'en manque un autre" (fait tomber les blocs, donc cherche l'autre voiture ou bloc qui manque). Jean-François: 'T'as juste à n'en prendre un autre, là" (dans le casier de rangement). Jean-François: “Aie! Veux-tu que je te fasse ça?” (en parlant du devant de l'édifice qui s'écroule). "Encore!" (reconstruit l'édifice).

Pourtant, Jean-François se replace dans une position où il demande à l'autre la permission, donc tient-il compte de son rôle de décideur. Accepte-t-il partiellement sa position d'exécuteur?

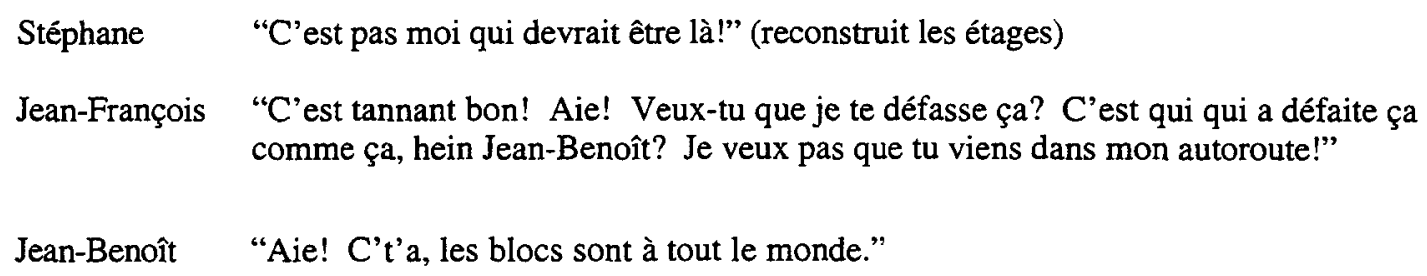

Dans sa réplique, Jean-François se sert-il d'une tierce personne comme stratégie de diversion de cette impasse, puisque cela ne semble pas évident à régler avec Stéphane. Et que malgré les différentes stratégies déployées, ils ne semblent pas trouver de terrain pour dénouer ce conflit? Ou bien, comme il y a peu de pouvoir ou de statut de décideur pour conduire le jeu, essaie-t-il de se donner un certain pouvoir sur Jean-Benoît du fait qu'il ne peut pas en avoir sur Stéphane?

Et Jean-Benoît le replonge automatiquement dans ce qui nous apparaît comme l'élément fondamental de l'évolution de cette dynamique, en mettant en évidence la question de droit "les blocs sont à tout le monde", pas plus à lui qu'à Jean-Benoît, et qu'il peut les utiliser.

À ce moment, Jean-François dit: "Je sais, mais c'est moi qui a faite ça, alors c'est moi qui décide!". Donc, il reconnaît le fait que les blocs sont à tout le monde, mais aussi que, puisque c'est lui qui a fait l'autoroute, ça lui donne des droits, entre autres celui de décider. Donc simultanément, il reconnaît à l'autre, Stéphane, le droit de décider, puisque c'est son édifice, mais Jean-François n'accepte pas cette situation et il ne reconnaît pas ce droit à l'autre, alors qu'il voudrait qu' on le lui reconnaisse. 
Jean-Benoît: "Mais tu sais, on a le droit d'être deux" (fait rouler sa voiture un bout sur l'autoroute et revient à sa construction) et Jean-François fait signe que non de la tête à Jean-Benoît. Probablement non, si lui n'a pas décidé du fait d'être deux.

Jean-François "Là-dedans, y'a pas d'autos, je crois que je sors de mon garage" (ouvre la porte).

Stéphane T'as pas le droit."

Ces répliques soulèvent une fois de plus les différents droits de chacun dans cette situation.

Stéphane: "Moi aussi, je sors de mon garage" (défait les devantures du garage à étages). Stéphane, dans sa réplique, vient de signifier que Jean-François n'a pas le droit de sortir de son garage et immédiatement après, il signifie que lui aussi il sort de son garage. Donc, qu'il s'attribue le droit de sortir! Encore une ambiguïté sur le droit. JeanFrançois ajoute: "Pour aller dans l'autoroute, même moi je sors de toutes les garages", en signifiant à Stéphane qu'il se dirige vers l'autoroute qui est sa construction et qu'il est en droit de décider.

Jean-François

Stéphane
"T'avais pas lui, moi je voulais."

“Aie! Après qui s'tienne? Aie! Ça a tombé encore! C'était même pas celle-là! je commence à être tanné."

“Aie! Prends-les tes autos!" (fait rouler sa voiture sur l'édifice).

Donc, comme il utilise ses autos pour se diriger vers sa construction, il lui accorde ce droit de prendre ses autos à lui et de faire ce qu'il veut!

Jean-François "C'pas là-dedans, c'est pas ceux-là!"

Stéphane "On peut monter là-dedans comme ça" (en imitant le bruit d'une voiture qui force en montant) "Je pouvais construire ici."

Jean-François "Regarde que ça fait! Ça tombe tout le temps à cause de c'ta porte de garage-là" (reconstruit le garage en plaçant ses portes)

Stéphane "J'ai une idée!"

Est-ce que l'ambiguité des droits et des statuts de chacun est rompue du fait que chacun est retourné à sa construction et que là, Stéphane vérifie la possibilité d'un terrain réciproque d'entente, donc il risque de lui faire part d'une idée? 
Jean-François répond "Non!" mais Stéphane élabore tout de même son idée: "Tu merras quelque chose d'autre" (referme les portes du garage à étages). Stéphane acquiesce à sa proposition en suggérant lui aussi une structuration du déroulement du soidisant nouveau terrain d'entente. "Moi je prends celle-là du bas" et sur ce Stéphane répond: "Non! Ah! Ah! Non on peut pas (ça tombe encore) Aie! Regarde c'est quoi que t'as faite."

Décidément, on revient à la case départ quand l'un ou l'autre des partenaires veut prendre une décision pour le déroulement du jeu et des gestes à poser. Sont-ils en train de requestionner les statuts de chacun?

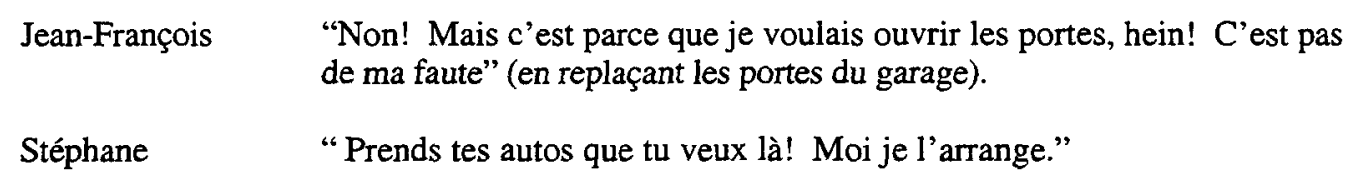

Donc, revient-il sur ses droits parce que c'est son édifice? Jean-François répond finalement: "Aie! C'est moi qui l'arrange, lâche-les (le bloc, la porte) c'est à cause de toi qui le tiens, ça fait toute tomber." Et les voilà repartis sur les mêmes bases conflictuelles.

- Événement de désaccord inter-dyade

La séance 8 présente un événement inter-dyade de désaccord qui survient dans l'activité de jeu symbolique. Dans cette séance, les enfants ont recours à l'enseignante pour dénouer un désaccord.

Frédéric et Simon entrent ensemble dans l'aire des blocs. Frédéric signifie son besoin en termes de matériel et Simon lui propose son intention de projet global à deux. Frédéric accepte cette proposition. Neuf minutes plus tard, Véronique et Audrey arrivent dans l'aire des blocs. Audrey propose une intention de projet à deux. Elles discutent des différentes façons de faire et s'entendent finalement sur un projet global de construction, "un château".

Dans cette séance, 19 événements inter-dyades se produisent. Voici la nature de chacun de ces événements. 


\begin{tabular}{|c|l|c|}
\hline Événement & Objet de l'interaction & Initiateur de l'événement \\
\hline $1^{\text {er }}$ & Partage du matériel & Simon \\
\hline $2^{\text {er }}$ & Échange du matériel & Audrey \\
\hline $3^{\text {er }}$ & Proposition du matériel & Simon \\
\hline $4^{\text {er }}$ & Demande d'informations à la chercheuse & Simon \\
\hline $5^{\text {er }}$ & Demande d'informations sur le projet global & Émilie \\
\hline $6^{\text {er }}$ & Échange de matériel & Audrey \\
\hline $7^{\text {er }}$ & Intervention de la chercheuse & Chercheuse \\
\hline $8^{\text {er }}$ & Demande de partage de matériel & Simon \\
\hline $9^{\text {er }}$ & Don de matériel (animal) & Véronique \\
\hline $10^{\text {er }}$ & Avertissement & Audrey \\
\hline $11^{\text {er }}$ & Échange de matériel & Véronique \\
\hline $12^{\text {er }}$ & Don de matériel (courbe elliptique) \\
\hline $13^{\text {er }}$ & Infiltration dans un échange sans demande d'avis & Audrey \\
\hline $14^{\text {er }}$ & Informations sur le projet de construction & Simon \\
\hline $15^{\text {er }}$ & Proposition d'idée à l'autre dyade & Frédéric \\
\hline $16^{\text {er }}$ & Demande d'explications & Frédéric \\
\hline $17^{\text {er }}$ & Proposition de matériel & Audrey \\
\hline $18^{\text {er }}$ & $\begin{array}{l}\text { Destruction du projet global de construction } \\
\text { de l'autre dyade }\end{array}$ & Audrey-Véronique \\
\hline $19^{\text {er }}$ & Intervention de l'enseignante & \\
\hline & & \\
\hline
\end{tabular}

Dans cette séance, un premier événement de désaccord s'initie entre les dyades par une réplique d'avertissement initiée par les deux filles pour que les garçons ne démolissent pas leur production.

Audrey "Faisez attention pour pas démolir notre petit château!" (aux deux garçons)

Simon 'Ben, c'est pas un château ça! C'est ben trop grand! Sur la butte, regardez ousque que vous allez avec votre château. Y'é quasiment démoli, vous le démolissez."

“Hein!"

“Aie! Regarde-là à cause de vous autres." 
Par cette réplique, les garçons essaient de retourner leur responsabilité aux filles, en les accusant que c'est leur faute.

Audrey "Ben nous autres, on a rien faite".

Audrey resitue les responsabilités des actes de chacun.

Simon "Pis vous prendrez toutes les blocs."

Au départ, la discussion s'est amorcée sur un avertissement pour ne pas détruire, mais là Simon semble discuter sur le fait que les filles prennent tous les blocs. Audrey répond que les blocs qu'elles prennent, c'est parce qu'elles en ont besoin.

Frédéric "Au moins, nous autres, on va toutes les prendre, hein!"

Donc ici, Frédéric reprend exactement pour eux-mêmes, ce qu'il reproche aux filles. Une ambiguïté apparaît donc sur le plan de l'échange entre les membres de cette séance.

Simon propose: "Ouais! Prenez-en encore mais pas trop", il s'accorde le droit de prendre tous les blocs et leur donne la permission d'en prendre, mais pas trop. Mais Audrey n'est pas d'accord et ne comprend probablement pas l'ambiguité soulevée. "Aie! Les blocs, c'est pour tout le monde, on peut les prendre an qu'on veut".

Audrey soulève les droits de modalité de fonctionnement et la propriété à tous des blocs. Mais plutôt que d'éclaircir et de rétablir l'équilibre sur cette ambiguité de l'échange, Simon continue en changeant de plan et se moque d'Audrey sur le plan personnel au niveau de son langage:

Simon 'An qu'on veut! Pas an! Quand qu'on veut! Ah, an c'est quand? Quand qu'on veut, pas an qu'on veut."

Audrey "Pouah! C'est ça qu'on dit, hein!"

Donc, elle ne semble pas vouloir poursuivre dans cet échange et lui signifie que c'est correct ce qu'elle dit. Cependant, Frédéric reprend la réplique et confronte Simon en renforçant et en reprenant l'expression d'Audrey: "An qu'on peut" et en se moquant de cette expression: "An qu'on peut". Et là, elle tente de trouver une stratégie pour amener la 
rupture et cesser cet échange, mais tout ce qu'elle trouve c'est: "Tu savais même pas comment parler". Frédéric: “Ouais! Je t'avais compris. Aie! Sais-tu c'est quoi tu m'avais dit? Tu m'avais dit là que je me pense bon mais toi tu te penses bonne?"

Est-ce que Frédéric fait référence à un échange antérieur avec Audrey? Et, si cet échange ne s'est pas réglé, est-ce qu'il profite de celui-ci, comme une réminiscence de cet échange non résolu?

Finalement, leur échange a débuté sur un avertissement de non-destruction, a glissé sur les droits de l'aire des blocs et à la propriété des blocs à tous, a continué à glisser sur la moquerie d'une expression qu'Audrey a utilisée et finalement fait ressortir la position de chacun dans la relation. Et puis, Véronique recentre le tout sur "Fais attention".

Est-ce que c'est le même avertissement de départ à propos de la construction? Est-ce que c'est un avertissement à propos du terrain de négociation qu'il vient de lancer? Finalement, Frédéric se recentre sur son activité de jeu symbolique, amorcée avant l'échange.

Un dialogue s'engage sur le plan symbolique entre les garçons. Ils discutent des animaux et des personnages-figurines. Un des enfants propose que la figurine ait le rôle de patron et l'autre propose que ce soit d'autres figurines qui personnifient ce rôle. Alors, Audrey, un enfant de la dyade des filles, intervient: en adoptant la position de celui qui a fait la première proposition et qui acquiesce positivement à cette proposition externe. L'autre enfant réagit vivement: “Ouais! Mais je veux dire quelque chose. Si t'arrêtes pas de blaguer, je t'envoye mon armée (et Audrey veut lui couper la parole avant même qu'il ait fini de présenter son idée). Bon voudrais-tu te mêler de tes affaires, S.V.P. Si t'arrêtes pas de blaguer, je vas t'envoyer mon armée."

Audrey "Aie! Y blague pas."

Par cette réplique, Simon veut, de nouveau, expliquer son point de vue à son partenaire en ramenant l'idée de ce dernier sur un plan transitoire entre le symbolique et la réalité, en parlant de blague, et il revient sur un plan symbolique, en lui proposant d'envoyer son armée. Cependant, Audrey insiste et veut prendre la parole. Alors, à ce moment, Simon revient sur le plan de la réalité et adopte une position explicite claire et suggère po- 
liment à Audrey de se mêler de ses affaires. Il lui renvoie explicitement sa situation externe à la dynamique ludique qui concerne lui et Frédéric seulement, et qu'ils n'ont pas besoin d'elle pour décider de quelque chose qui ne la concerne pas. Et, il revient simplement sur le plan symbolique pour réitérer sa position à son partenaire en lui répétant que s'il n'arrête pas de blaguer, il va envoyer son armée.

Malgré cette position explicite à l'égard d'Audrey, celle-ci ajoute que Frédéric ne blague pas. Mais Frédéric répond à Simon sur le plan symbolique "d'envoyer son armée" et Simon continue cette lancée sur le plan symbolique.

Ici, cet événement présente assez clairement la compétence de Simon à alterner d'un plan symbolique à un plan de la réalité et fait apparaître clairement sur le plan de la relation interpersonnelle la position qu'Audrey occupe. Il lui renvoie l'image explicite de la perception de rôle dans cette situation ludique.

Une autre interaction avec un déroulement qui accentue le désaccord survient à la dyade des filles et celles-ci ont recours à l'enseignante pour dénouer le désaccord et possiblement rétablir l'équilibre.

Dans le vif de leur activité de jeu symbolique, les garçons sont dans une séquence où les animaux attaquent le château et le détruisent, des personnages-figurines sont morts et d'autres blessés.

À ce moment, au coeur de leur action, Simon, avec un personnage-figurine en bois jaune, détruit un meuble de la maison des filles. Alors les garçons s'encouragent, rient et détruisent le château, production de la dyade des filles.

40:55:18 "Aie! Y détruit toutes les choses, y détruit toute, toute, toute eux autres."

41:05:15 “Aie! Dis au maudit bonhomme qui s'en aille de d'là! Dis à ton petit pitou qui s'en aille."

La réplique de Audrey utilise le plan symbolique pour faire passer son message de destruction. 
Le message se situe à deux niveaux. Au premier niveau, Audrey respecte l'activité de jeu symbolique en cours des garçons et reconnaît que la destruction fait partie du déroulement du scénario. Mais simultanément, elle passe à un autre niveau le message aux garçons en leur demandant de contrôler leur personnage et de s'en aller.

Là, Frédéric intervient et donne une réplique à Audrey qui le renvoie sur un plan personnel: “Audrey, dis à ta petite Véronique qu'à s'en aille. Aie! Regarde! Y'a des seins!" (le gorille).

Et Simon continue de détruire le château des filles: "Aie! Je détruis votre château. Je détruis toute". À ce moment, les filles vont chercher l'enseignante pour qu'elle intervienne au niveau du conflit et Simon ajoute: "Vous avez le droit" (parce que les filles sont allées chercher l'enseignante). Et Frédéric dit à Simon, comme pour prendre les devants: "Viens, on va le dire à notre enseignante".

Après l'intervention de l'enseignante, les enfants reprennent leur jeu à la suite de l'idée proposée par l'enseignante. Cependant, après quelques minutes, la problématique de départ à propos de la destruction de la production est soulevée de nouveau, aussitôt que les enfants recommencent à jouer ensemble. Les enfants se retrouvent au coeur de l'action, le désaccord resurgit avec la même intensité.

Ainsi, la problématique soulevée au début de la séance revient en force, ce qui indique la possibilité que les moyens retenus n'ont pas résolu de façon satisfaisante la situation conflictuelle. Est-ce que la vraie problématique a été identifiée? Est-ce que les enfants ont exprimé leurs sentiments et leurs émotions relatifs à cette situation? Est-ce que différents moyens de résolution ont été exploités? Est-ce que ces moyens correspondent aux choix des enfants? Un grand nombre de questions peuvent être envisagées pour tenter de comprendre les éléments de cette séquence présentant une situation conflictuelle.

Malheureusement, l'intervention de l'enseignante ne permet pas une résolution fructueuse du conflit entre les enfants et ce pour différentes raisons.

Au départ, la requête des filles sur la destruction du château situe la position de l'enseignante à partir de la seule version des filles. Il nous apparaît important, dans un premier mouvement, de rétablir les faits de la situation conflictuelle à partir des versions de 
chaque membre des dyades. Ainsi, les différents éléments de la compréhension du conflit sont rendus explicites et posent les bases possibles d'une résolution éventuelle de la situation. L'ouverture de l'enseignante à recevoir la version de chacun permet d'établir une position claire de médiateur dans la situation conflictuelle en évitant ainsi de prendre parti pour l'une ou l'autre des équipes.

Cette première attitude d'écoute des différents faits, amène chacun des membres à exprimer leur perception et déjà à poser les éléments importants qui constituent les vraies intentions de chacun des enfants dans la situation conflictuelle.

Une deuxième attitude importante d'intervention se situe sur le plan du questionnement. L'enseignante doit poser des questions qui recontextualisent les événements de la situation conflictuelle. Par la suite, son questionnement peut être orienté pour permettre l'expression des sentiments et des émotions de chacun des enfants dans cette situation. Les enfants peuvent donc apprendre également à s'exprimer sur un plan plus affectif et apprennent à tenir compte des sentiments et des émotions des autres et que ceux-ci peuvent être tout à fait différents des leurs. De plus, il est important que chacun dispose de suffisamment de temps pour exprimer ce qu'il a à dire. Dans cette attitude d'écoute, l'enseignante donne du temps à chacun afin de bien établir la problématique de la situation et ce que chacun ressent dans cette situation conflictuelle.

Une fois que les éléments de la situation conflictuelle sont établis, l'enseignante peut amener les enfants sur différentes pistes de moyens possibles à mettre en oeuvre pour dénouer la situation conflictuelle. Dans un premier mouvement de recherche de solutions, il est intéressant que les enfants aient la possibilité d'énoncer des moyens qu'ils connaissent. Par la suite, l'enseignante peut, avec eux, explorer d'autres moyens. Il est important que l'enseignante demeure, tout au long de la démarche d'intervention, dans son rôle de médiateur afin que chaque enfant puisse se positionner dans la situation sans prescription directe de sa part. Il est souvent difficile pour l'adulte, dans des situations conflictuelles, de demeurer dans son rôle de médiateur et de laisser les enfants trouver leurs propres moyens de résolution des conflits. On croit souvent comme adulte, que nos moyens susceptibles de régler avec satisfaction les conflits sont les meilleurs moyens pour les enfants. Est-ce que les moyens des enfants peuvent être différents et satisfaisants pour eux, dans la situation? Est-ce que la satisfaction de l'adulte ou des enfants qui est mise, ici, en 
évidence? Car la position occupée par l'adulte influence grandement le choix des enfants, et il est important que l'adulte prenne conscience de cette position.

Cette séquence fait donc ressortir la responsabilité de l'enseignante comme intervenante sollicitée dans la démarche de résolution d'une situation conflictuelle. De plus, cette séquence permet de questionner son propre mode d'appréhension des situations conflictuelles, en tant qu'adulte, et nous renvoie le miroir de nos propres stratégies et de nos propres moyens de résolution des situations conflictuelles. Dans le rôle que l'adulte joue comme intervenant, il devient donc primordial de développer nos stratégies d'aide, d'écoute, de questionnement, bref, de médiateur afin que les enfants développent à leur tour, leurs propres stratégies de résolution des conflits afin qu'ils deviennent de plus en plus autonomes et responsables dans les multiples situations auxquelles ils auront à faire face.

En conclusion, toute notre démarche d'analyse des données s'est traduite à travers des va-et-vient de mises en forme des données, d'analyses et de nouvelles mises en forme. Différentes pistes et mouvements d'interprétation se sont infiltrés, au fur et à mesure de la démarche d'analyse et de compréhension. Toutefois, afin de dégager l'essentiel de cette étape d'analyse, nous avons réalisé une synthèse des éléments s'inscrivant soit dans le prolongement de ce qui avait déjà été investigué dans les recherches consultées ou soit dans l'émergence de données issues de nos observations et de nos analyses.

Le chapitre suivant ne constitue donc pas la discussion des résultats dans une démarche plus traditionnelle de recherche. Il se veut le reflet de notre démarche de questionnement, l'enchaînement de plusieurs regards posés sur l'objet de recherche et un tremplin à l'ouverture de nouveaux regards sur le plan de la recherche et de l'intervention pédagogique. 


\title{
CHAPITRE V
}

\section{D'UN LIEN THÉORIQUE DE L'ANALYSE DES DONNÉES \\ À L'ÉMERGENCE D'UNE COMPRÉHENSION ET À \\ L'OUVERTURE DE NOUVEAUX REGARDS \\ POUR LA RECHERCHE \\ ET L'INTERVENTION PÉDAGOGIQUE}

\begin{abstract}
Dans le cadre du présent chapitre, nous rendrons compte, en les résumant, des résultats de l'analyse des données relatives aux trois questions de recherche de telle sorte qu'il soit possible de se rappeler la nature des activités que le matériel bloc suscite chez l'enfant et de savoir si ces activités contribuent au développement cognitif et social de l'enfant qui fréquente la classe maternelle. Ce dernier chapitre nous amène à poser un regard réflexif et critique sur l'ensemble de la démarche réalisée. Pour ce faire, nous retracerons le fil conducteur de cette étude à partir de l'objet de recherche constituant la problématique jusqu'à l'analyse des données. Par la suite, nous mettrons en évidence la portée de ces résultats sur le plan de la recherche dans le domaine des dynamiques de jeu et sur le plan des interventions pédagogiques.
\end{abstract}

La démarche poursuivie jusqu'à maintenant, dans notre questionnement sur les potentialités du matériel, les composantes et les produits liés à la nature et à la structure de l'activité de l'enfant avec le matériel bloc, a ouvert de multiples possibles quant aux différents regards à porter sur les données issues de nos observations. Nos choix, tout au long de cette démarche ont été soutenus théoriquement à travers un filtre constructiviste et socioconstructiviste. Les aspects individuel et social de ces approches se chevauchent, s'imbriquent et se coordonnent pour ouvrir des voies et des pistes de compréhension de l'apport et du support au développement cognitif et social de l'enfant et de son activité avec le matériel bloc. Les fondements de ces approches quant à la conception du déve- 
loppement de la connaissance, du développement de l'enfant, du jeu et du jeu symbolique constituent les bases conceptuelles qui ont servi de filtre à l'appréhension de l'activité de l'enfant avec le matériel bloc.

Ce dernier chapitre tente donc de positionner théoriquement les résultats d'analyse des données liés aux différentes questions de recherche; de dégager en quoi ces résultats s'inscrivent dans la même lignée que ceux des recherches antérieures; en quoi ils apportent des éléments complémentaires susceptibles de raffiner notre compréhension de l'activité de l'enfant avec le matériel bloc et finalement, de quelle façon ces résultats de recherche élargissent le champ de connaissances, si fragmentaires des dynamiques de jeu des enfants.

Nous tentons donc, une incursion au coeur de ce qui apparaît fournir des données complémentaires et émergentes dans une démarche plus qualitative de la cueillette et de l'analyse des données. Tout cela, afin d'établir des liens théoriques qui conduisent à l'exploration de pistes éventuelles de recherche et d'interventions pédagogiques.

\subsection{Résultats relatifs à l'analyse des données de la première question de recherche}

La première question de recherche renvoie au stade d'évolution de l'activité de construction. Les travaux menés par Johnson (1974) ont identifié certains procédés de construction correspondants à des stades ou étapes d'évolution de l'activité de construction. Rappelons également que d'autres chercheurs ont complété et raffiné ces stades d'évolution. Dans ces recherches, ces stades ont été élaborés à partir de photographies et de croquis des productions des enfants.

Pour investiguer notre première question nous avons, en plus de considérer les productions des enfants, pris en compte les actions sur le matériel et la communication verbale tout au cours du déroulement de l'activité de l'enfant avec le matériel bloc. L'ouverture de l'observation à d'autres éléments indicateurs de l'activité de construction a permis d'apprécier une activité de construction dans ses différentes étapes de réalisation ayant comme aboutissement les productions finales. Ce type d'observation, en plus de mettre en valeur les différents procédés de base et les multiples agencements et combinaisons de ces procédés, a permis, d'une part, de raffiner et de compléter les informations de 
l'ensemble des habiletés et compétences de l'enfant sur le plan technique et d'autre part, de saisir de façon plus substantielle la dynamique interne de l'activité de construction.

Les réponses à la première question de recherche devaient nous permettre de situer l'enfant de cinq ans qui fréquente la classe maternelle, à un stade d'évolution de l'activité de construction. Pour ce faire, nous avons analysé des données d'observation à partir de quatre thèmes de réflexion: les données liées aux stades d'évolution de l'activité de construction; aux produits de cette activité; au temps consacré à l'activité de construction et enfin, aux données relatives à la forme de participation sociale.

Chacun des thèmes de réflexion met en évidence des éléments caractéristiques émergeant de l'activité de construction et apporte des données nouvelles qui viennent enrichir, raffiner et approfondir la compréhension de l'activité de construction des enfants de cinq et six ans.

\subsubsection{Synthèse des résultats relatifs aux stades d'évolution de l'ac- tivité de construction}

Les données relatives aux stades d'évolution de l'activité de construction traduisent donc la présence, chez l'enfant de classe maternelle, d'habiletés et de compétences techniques et communicationnelles. Concernant les habiletés et les compétences techniques dans la réalisation des diverses productions avec le matériel bloc, les enfants manifestent une bonne maîtrise et flexibilité à utiliser, répéter, agencer et combiner les divers procédés et techniques de base de la construction. En effet, ils acquièrent individuellement et par l'apport significatif de l'autre comme partenaire de jeu, des procédés de base de construction et des différents agencements possibles. Cela leur permet d'acquérir, de perfectionner, de confronter et de partager certaines habiletés et compétences techniques pour devenir soi-même plus compétent et aussi devenir ensemble plus compétents.

Cet ensemble d'habiletés et de compétences, d'un point de vue technique, s'observe dans plusieurs situations:

- L'utilisation d'un nombre considérable de blocs (60 et plus) de toutes formes, grandeurs et longueurs pour chaque projet initié. 
- Une maîtrise des différents procédés de l'activité de construction à travers:

- l'utilisation des procédés de tour, de rangée, de pont et d'enclosure comme paliers de base à une multitude d'arrangements des plus variés les uns que les autres et qui font appel à la répétition et à la complexification de ces procédés de base pour l'élaboration de productions de plus en plus inusitées et sophistiquées dans:

- l'utilisation et la répétition des procédés de base de la tour et de la rangée dans la complexité d'une production;

- l'utilisation et la répétition du procédé de base du pont pour la réalisation d'une production à plusieurs étages;

- l'utilisation et la répétition du procédé de base de l'enclosure de plus en plus élaboré pour réaliser divers espaces d'une même production;

- l'utilisation de blocs tels triangle, carrefour, cylindre, chaussée, quart de cercle, Y connecteur, courbe elliptique, dans plusieurs productions comme éléments décoratifs;

- l'utilisation simultanée et combinée des divers procédés de construction (tour, rangée, pont, enclosure) dans l'élaboration de productions plus complexes.

- Le souci de l'enfant à ériger les parties de sa production en respectant les composantes de symétrie et d'équilibre, lequel se traduit:

- physiquement: dans les actions de l'enfant par les diverses manipulations du matériau bloc pour une reproduction exacte du modèle initié et ce, alternativement pour chacun des côtés de sa production; dans l'utilisation des mêmes types de blocs ou par équivalence pour ériger en hauteur, en largeur la production; dans l'utilisation des mêmes éléments décoratifs en symétrie;

- verbalement: dans les énoncés de son action de construction:

$\ll 1,2,3,4$ (d'un côté); $1,2,3,4$, (de l'autre côté), comme ça, comme ça, ton mur faudrait t'a r'mette, faudrait, à côté un bloc, faudrait l'a remettre un autre bloc, faudrait t'en remettre un autre (en montrant le mur, il regarde s'il y a le même nombre de blocs qui forme la partie avant de sa construction). Equivalence: Ah! on a pu, oh ben tend une minute, on va mettre ceux-là de même, donne-moi dont ceux-là, ça va être [...]. (Il place quatre blocs sur les murs afin d'obtenir une rangée de plus pour que les deux murs soient de la même hauteur).» 
En ce qui a trait aux habiletés et compétences communicationnelles sur le plan verbal, on peut affirmer que ces habiletés et compétences se distinguent par leur contenu selon les diverses formes de participation sociale et les interactions sociales qui s'établissent pendant l'activité de construction. Ainsi, les énoncés verbaux changent si l'activité de construction est parallèle ou de type associatif.

Dans une activité de construction parallèle, les énoncés verbaux des enfants ont été signifiés:

- pour faire part de leur intention de projet aux autres et à lui-même;

- pour donner une appréciation sur sa propre production;

- pour donner une appréciation sur la production d'un pair;

- pour demander du matériel;

- pour indiquer un ajustement;

- pour demander de l'aide;

- pour une mise en garde;

- pour le respect des règles;

- pour proposer une idée à un pair;

- pour entrer en interaction par le biais du matériel;

- pour verbaliser le processus d'élaboration de la production ou du scénario;

Dans une activité de construction de type associatif, les énoncés des enfants ont été signifiés:

- pour annoncer l'intention du projet, mais sans nécessairement qu'il y ait entente des partenaires pour son élaboration. Chacun peut réaliser son intention sur la même base de construction;

- pour inciter l'autre comme partenaire dans son activité de construction;

- pour demander conseil à l'autre;

- pour décrire chacune des actions dans l'élaboration de la production, donc pour verbaliser la démarche.

\subsubsection{Synthèse des résultats relatifs aux produits de l'activité}

Les résultats relatifs aux produits de l'activité sont les conséquences des trois tâches spécifiques observées lors de l'utilisation des blocs dans l'activité de construction: 1) la 
création d'un contexte, 2) la transposition des idées/la négociation avec la réalité et 3) la distanciation comme processus.

Ces résultats révèlent que les enfants savent utiliser une combinaison d'habiletés et de compétences techniques, cognitives et sociales pour créer un contexte de base, pour transposer leurs idées sur le plan opérationnel par l'intermédiaire du matériel bloc, et pour se distancier de soi-même soit pour s'investir comme acteur, soit pour s'identifier à un personnage-figurine.

Les données associées aux produits de construction en fonction de la première et de la deuxième tâche de l'enfant dans son activité de construction traduisent un certain nombre d'éléments permettant de tracer une constance dans les modalités d'élaboration du contexte.

En premier lieu et d'une façon générale, ces données ont mis en évidence la variété de productions susceptibles d'être réalisées à l'aire des blocs, un ensemble d'intérêts et de préoccupations tributaires du développement, des connaissances et des expériences individuelles et sociales des enfants, et un ensemble d'habiletés et de compétences dans la réalisation de ces productions. En effet, ces enfants manifestent une plus ou moins grande aisance et flexibilité à utiliser, répéter et agencer les divers procédés techniques de base afin de représenter, par l'intermédiaire des blocs, une idée du contexte.

En deuxième lieu et d'une façon particulière, ces données sur la variété des productions ont permis de dégager un ensemble de projets globaux qui sont des projets pivots constituant le contexte de base de l'activité de construction. Ces projets globaux regroupent, dans cette étude, quatre grandes catégories de production: des productions représentant (1) différents genres d'édifices; (2) des moyens de transports sur mer, terre et air; (3) des systèmes routiers simples et complexes et (4) diverses productions apparaissant moins souvent dans l'activité de construction mais tout autant significatives. Ce contexte de base sert l'activité de construction. C'est un contexte matériel qui permet l'activité de construction et l'oriente pour déclencher une activité de jeu symbolique.

De plus, autour des projets globaux gravitent un ensemble de projets secondaires et complémentaires qui se réalisent selon deux modalités, la première, de l'ordre du mode de 
transformation matérielle et la deuxième, de l'ordre du mode de transformation symbolique.

En troisième lieu et d'une façon particulière, les intérêts et les préoccupations manifestés par les enfants ont permis de dégager un ensemble d'intérêts communs aux enfants de ce groupe d'âge. En effet, le matériel utilisé, soit les blocs, suggère non seulement l'activité de construction, mais aussi des modèles à construire. En effet, les enfants utilisent le matériel pour construire des maisons, des moyens de transports, des routes etc, c'est à dire des éléments de leur environnement.

Finalement, la tâche de distanciation de soi s'est réalisée selon deux types de structures: une première s'élabore à la dimension de la taille des enfants et une deuxième à la dimension de la taille des figurines. L'un et l'autre des types d'élaboration des structures entraînent une implication et un investissement très différents de l'enfant dans l'activité de construction. Il faut noter que les enfants qui construisent leur structure aux dimensions de leur taille annoncent verbalement leur intention et ce, dès les premières minutes de l'activité.

\subsubsection{Synthèse des résultats relatifs au temps consacré à l'activité de construction}

L'analyse des données concernant le temps consacré à l'activité de construction montre que l'ensemble des enfants consacrent 59\% du temps total à l'activité de construction et $39,5 \%$ du temps à l'activité de jeu symbolique. Cependant, les filles investissent en moyenne $70 \%$ du temps total à l'activité de construction et $23 \%$ à l'activité de jeu symbolique, tandis que les garçons investissent $52 \%$ du temps à l'activité de construction et $45 \%$ du temps à l'activité de jeu symbolique. En l'occurrence, l'activité de construction constitue la première activité des enfants à partir de laquelle sont produites différentes structures (35/36 enfants, 9 séances) et l'activité de jeu symbolique est prioritairement une activité subordonnée à l'activité de construction.

Cependant, quelques séances (3) montrent que l'activité de jeu symbolique est simultanée à l'activité de construction mais, cette activité s'élabore toujours à partir d'un projet global de construction et, par la suite, les deux activités s'influencent mutuellement. 


\subsubsection{Synthèse des résultats relatifs aux diverses formes de partici- pation sociale}

Finalement, l'analyse des données relatives aux diverses formes de participation sociale indique que les enfants interagissent en mode solitaire, dyadique et à quatre membres. Le mode de regroupement dyadique est le mode de participation sociale le plus fréquemment adopté par ces enfants. En deuxième lieu, apparait les modes solitaire et parallèle. Quant au mode de participation sociale à quatre, il a été peu observé dans cette étude, car il implique des modalités d'interactions sociales plus complexes et plus développées afin d'assurer la viabilité d'un projet collectif de construction.

\subsection{Résultats relatifs à l'analyse des données de la deuxième question de recherche}

La deuxième question de recherche voulait mettre en évidence la préoccupation quant au jeu symbolique suscité par l'activité de l'enfant avec le matériel bloc. En effet, l'observation de l'activité de l'enfant laisse apparaître divers éléments constitutifs du jeu symbolique, des moins complexes aux plus élaborés. Cependant, n'ayant aucun doute sur les possibilités symboliques de cette activité, les interrogations se situent non seulement sur la présence de ces éléments symboliques, mais aussi et surtout, sur leur nature puisque l'ensemble des auteurs qui ont élaboré les stades d'évolution de la construction ont observé, chez les enfants de cinq-six ans, l'apparition d'une forte impulsion au jeu dramatique à partir et autour des productions. Il est intéressant d'examiner la ou les spécificités des composantes structurales constituant l'activité de jeu symbolique à partir du matériel bloc.

Ainsi, la deuxième question de recherche voulait déterminer s'il y a similitude et/ou spécificité de l'activité de jeu symbolique à partir du matériel bloc et s'il y a spécificité, quelles sont les composantes structurales de cette activité.

L'identification des épisodes reflétant des éléments de jeu symbolique supposait, dans un premier mouvement d'analyse, que le relevé de l'ensemble de ces éléments devait se réaliser a priori seulement dans l'activité de jeu symbolique simultanée ou subordonnée à l'activité de construction. Cependant, cette analyse a révélé la présence d'un ensemble d'éléments de jeu symbolique à l'intérieur même de l'activité de construction. Ainsi, la 
présence et la nature des composantes structurales du jeu symbolique se devaient d'être également examinées dans l'activité de construction. Cette première constatation se réfère à la nature même du matériel bloc comme objet de jeu étudié au premier chapitre.

Rappelons que nous avons mis en évidence que le matériel bloc se définit en fonction de son caractère permanent, lié de façon intrinsèque à sa matérialité. Puisqu'il demeure jouet, même en dehors du jeu, le bloc, comme objet de jeu, possède des caractéristiques internes et un potentiel propre. Ainsi, il suscite le jeu qui devient le prolongement de l'usage de l'objet. Les blocs ont un potentiel latent intrinsèque qui se traduit à travers l'activité de construction de l'enfant.

Selon une approche sociale, Brougère (1984b) définit le jouet comme média culturel, un objet support de représentations culturelles dans lequel s'inscrivent les divers sens culturels et sociaux. Comme un support, il est un instrument de jeu qui donne les moyens d'accéder à certaines formes de jeux culturellement déterminées et il est un moyen de transmission de contenus culturels. Toujours selon cette approche, le jouet est constitué de deux dimensions importantes: la dimension matérielle et la dimension symbolique. Ces deux dimensions définissent, à leur tour, les aspects statique et dynamique du jouet.

Avant même que l'enfant n'intervienne sur ce matériel, les blocs eux-mêmes demeurent des formes simples et sans représentation qui, par contre, orientent déjà dans leur matérialité le genre de productions que les enfants peuvent réaliser. Communément, ces blocs guident et soutiennent la réalisation de différents genres d'édifices (maisons, châteaux, garages, hôpitaux), de divers moyens de transport (bateaux, avions, camions, autos, traversiers), de systèmes routiers variés et complexes etc., comme l'ont révélé nos observations.

Encore sous son aspect statique, mais cette fois-ci dans sa dimension symbolique, l'intervention de l'enfant prend tout son sens. À un premier niveau de représentation, l'enfant, à partir des différentes pièces de bois, peut représenter autant d'univers familiers que d'univers imaginaires, autant d'univers imprégnés dans la matérialité des blocs euxmêmes. 
C'est sous cet aspect statique et à ce premier niveau de représentation de la dimension symbolique que s'inscrit l'activité de construction. Le matériel bloc, comme un autre objet, renvoie à l'évocation de quelque chose de réel dans l'environnement, seulement, et seulement si l'enfant le manipule et l'assemble pour constituer son entité représentationnelle. Le bloc n'est pas une entité constituée et complète de sa représentation comme l'est une poupée ou un camion, l'enfant doit agir sur le matériel pour lui donner une représentation externe. C'est à notre sens ce qui explique la présence d'éléments constitutifs de l'activité symbolique. L'enfant doit créer les divers éléments du contexte qui lui permettront d'accéder au deuxième niveau de représentation sous son aspect dynamique.

À ce deuxième niveau, toutes les représentations élaborées à l'aide du matériel bloc donnent lieu à diverses productions qui peuvent devenir un élément moteur et déclencheur autour desquelles vont s'élaborer une infinité de jeux symboliques. Avec le matériel bloc, l'enfant est d'abord constructeur et ensuite scénariste et acteur.

Selon cette approche sociale, le bloc, comme objet de jeu, agirait différemment dans l'évolution du jeu des enfants dans les dimensions matérielle et symbolique. En fait, dans la dimension matérielle, l'enfant doit constituer une représentation externe, d'où la présence d'éléments de l'activité symbolique. Avant son activité de jeu symbolique, comme avec une poupée ou un camion, l'enfant doit d'abord créer la représentation externe à partir du matériel bloc.

L'enfant a donc un niveau supplémentaire de représentation à organiser, ce qui implique une connaissance et une expérience en lien avec le matériel utilisé, ici en l'occurrence les blocs, d'abord pour son activité de construction et, par la suite, par les productions réalisées pour son activité de jeu symbolique.

Examinons donc ce qui s'est dégagé de la présence et de la nature des éléments symboliques dans l'activité de construction et dans l'activité de jeu symbolique en prenant en compte les différentes formes de participation sociale. 


\subsubsection{Synthèse des résultats relatifs aux composantes structurales dans l'organisation solitaire}

\section{- Les substitutions d'objets}

Les résultats relatifs aux substitutions simples d'objets dans les dix organisations solitaires de l'activité de construction et de l'activité de jeu symbolique révèlent que même si dans certaines séances, le temps consacré à l'activité de construction est moins important que celui consacré à l'activité de jeu symbolique, la construction demeure dans toutes les séances l'activité de base sur laquelle s'élabore l'activité de jeu symbolique.

Les quelques substitutions symboliques simples d'objets sont des éléments initiatiques annoncés sans toutefois donner naissance à l'élaboration de scénarios mais, elles sont annoncées et, dans certains cas, exécutées dans le contexte de l'activité de construction pour alimenter et soutenir l'élaboration de cette activité.

Dans d'autres cas, certaines substitutions symboliques simples d'objets sont reprises par un autre enfant de l'aire des blocs, à titre imitatif, sans toutefois constituer une trame personnelle de sa propre activité de construction.

Finalement, des substitutions simples d'objets très isolées du contexte du projet global, c'est-à-dire sans lien avec le projet global, se réalisent sans s'inscrire de manière significative dans l'activité de construction de l'enfant.

\section{- L'adoption de rôles}

Dans cette forme individuelle et parallèle de réalisation des projets de construction, une autre composante structurale apparait, celle de l'adoption de rôles. Dans le jeu solitaire, les rôles éventuels sont tous considérés comme des rôles fonctionnels, et ils sont amorcés par le biais de paroles para-linguistiques ou d'onomatopées qui sont généralement en lien avec la manipulation spontanée d'un accessoire de jeu, tel les voitures ou en lien avec le projet global de l'activité de construction.

Quelquefois, il s'agit des gestes imitatifs d'un rôle social donné, comme celui de conducteur d'une voiture, lesquels ne sont pas explicités mais reconnus par l'observateur. 
Finalement, dans l'ensemble de ces projets individuels se dégage la présence d'un ou deux épisodes découlant prioritairement de l'activité de construction. Les rôles de ces épisodes sont des rôles à caractère de fiction comme le fantôme et des rôles à caractère stéréotypé comme un exploseur de dynamite et des voleurs.

\subsubsection{Synthèse des résultats relatifs aux composantes structurales dans l'organisation dyadique}

Les résultats relatifs à cette forme de participation sociale de la dyade ont révélé que l'ensemble de ces organisations présentent une activité de jeu symbolique subordonnée à l'activité de construction. En effet, les enfants élaborent des projets globaux et secondaires dans l'activité de construction et, par la suite, ces projets donnent lieu à l'élaboration de scénarios dans l'activité de jeu symbolique.

Certaines de ces organisations dyadiques montrent une activité de jeu symbolique simultanée à l'activité de construction; soit que l'activité de construction constitue toujours l'activité pivot, ou soit que l'apparition de l'activité de jeu symbolique se réalise sous trois formes d'agencements impliquant deux types de simultanéité.

Le premier type de simultanéité de l'activité de jeu symbolique à l'intérieur de l'activité de construction se présente sous deux formes d'agencements: la première est une simultanéité épisodique de courte durée de l'activité de jeu symbolique dans l'activité de construction, et la deuxième est une simultanéité épisodique de longue durée de l'activité de jeu symbolique dans l'activité de construction.

Le deuxième type de simultanéité est une simultanéité de l'activité de construction à l'intérieur de l'activité de jeu symbolique et se présente sous la forme d'une simultanéité épisodique de courte durée de l'activité de construction dans l'activité de jeu symbolique.

\section{- Les substitutions symboliques simples d'objets dans l'activité de jeu symbolique subordonnée à l'activité de construction}

L'organisation dyadique où l'activité de jeu symbolique est subordonnée à l'activité de construction révèle que les enfants élaborent des projets globaux et secondaires dans leur activité de construction et, par la suite, initient une activité de jeu symbolique à 
partir des différents projets réalisés. Ici, les substitutions symboliques simples d'objets (26) s'effectuent dans le contexte de l'activité de construction, et les substitutions symboliques dans l'élaboration d'un scénario (4) et celles à l'intérieur d'un scénario (28) s'élaborent dans le contexte de l'activité de jeu symbolique.

Ces substitutions symboliques simples d'objets semblent répondre spécifiquement à trois fonctions. La première fait référence à l'attribution d'une signification particulière à un bloc dans le but d'une représentation matérielle de l'objet, impliquant une transformation essentiellement symbolique et un partage de cette signification avec le partenaire de la dyade. La deuxième concerne l'attribution d'une signification particulière à un agencement de blocs dans le but d'identifier un espace spécifique dans la production et un partage de cette signification avec le partenaire de la dyade. Et la troisième fonction est constituée par l'attribution d'une signification particulière d'un bloc comme personnageanimal impliquant une transformation essentiellement symbolique et un partage de cette signification avec le partenaire de la dyade.

Finalement, certaines substitutions simples d'objets sont réalisées à partir du matériel accessoire. Celles-ci sont essentiellement une transformation symbolique et un partage avec le partenaire de la dyade.

- Les substitutions d'objets dans l'activité de jeu symbolique simultanée à l'activité de construction

L'organisation dyadique où l'activité de jeu symbolique est simultanée à l'activité de construction révèle que les substitutions symboliques simples, sont dans l'ensemble, réalisées dans l'activité de construction. Elles ne soutiennent pas les épisodes de l'activité de jeu symbolique simultanée à l'activité de construction, elles sont plutôt produites pour répondre à des besoins ponctuels dans l'activité de construction.

Quelques-unes de ces substitutions produites dans l'activité de jeu symbolique simultanée à l'activité de construction ont comme fonction d'alimenter et de soutenir les deux types d'activité et servent le scénario amorcé. Ainsi, les substitutions symboliques à l'intérieur du scénario sont des substitutions ponctuelles faites au fur et à mesure du développement du thème. Elles apparaissent en chapelet, fixent et relient les divers éléments du scénario. 


\section{- L'adoption de rôles dans l'activité de construction}

L'adoption de rôles est envisagée d'emblée dans l'activité de jeu symbolique, c'està-dire dans une trame impliquant tout un ensemble d'éléments constituant le jeu symbolique. Cependant, on a relevé, dans cette étude, plusieurs épisodes où l'adoption est utilisée également dans l'activité de construction. De cette manière, cette composante structurale indique que certains rôles périphériques et fonctionnels sont nommés et suscités par un élément de la situation et que leur fonction est neutralisante. En effet, le rôle neutralise l'action en cours (altercation de deux enfants) et permet la poursuite de ce qui a été commencé avant l'altercation.

D'autres rôles fonctionnels sont nommés et non joués dans l'activité de construction et sont en lien avec le projet global. Plusieurs fonctions leurs sont attribuées: une fonction de "traduction" dans le sens de représenter certains éléments de connaissance de l'enfant relatifs au thème de construction. Une deuxième fonction de partage de chacune de ces significations selon l'acceptation de l'autre pour la poursuite de l'activité. Finalement, une fonction de mise en place d'éléments constitutifs possibles d'une trame symbolique. Ces éléments et cette trame sont susceptibles d'être réinvestis dans des séquences ultérieures de l'activité de jeu symbolique.

Ainsi, tous ces rôles traduisent des éléments de connaissance qui supportent, alimentent, orientent et justifient l'activité de construction qui se réalise sur des éléments du réel, de l'environnement et de ce que l'enfant connaît de son environnement et des rapports qu'il établit .

\section{- L'adoption de rôles dans l'activité de jeu symbolique subordon- née à l'activité de construction}

L'ensemble des rôles, tantôt nommés, initiés, tantôt joués se regroupent sous les différents vocables identifiés par Garvey (1977), soit des rôles fonctionnels, familiaux, de caractère stéréotypé et de fiction, périphériques et fantaisistes. Nous avons observé plus de 45 rôles nommés, initiés et joués par les enfants. Ce sont surtout des rôles périphériques, de caractère de fiction jusqu'aux rôles de personnages -animaux en passant par tous les autres types de rôles. L'identification de ces rôles est présentée à l'appendice 
A-4. Un rôle émergent a été relevé, soit celui où l'enfant s'investit comme acteur dans un personnage-animal par l'intermédiaire de figurine-animal.

De plus, ces différents rôles nommés, initiés et/ou joués manifestent d'autres fonctions. Des rôles non joués dans une trame symbolique sont parfois nommés par l'intermédiaire de figurines. Ils ont comme fonction, l'élaboration d'éléments du cadre du thème du jeu, la négociation d'éléments du cadre du jeu, la traduction d'éléments de connaissance des rôles susceptibles d'être joués dans le thème exploité et l'identification du statut de l'enfant comme scénariste. Des rôles familiaux implicites sont initiés par la reproduction de comportements par des actions susceptibles de se réaliser dans différents espaces.

Des rôles de caractère stéréotypé, initiés par la reproduction de comportements, d'actions et de paroles inhérents à ces rôles ont été observés et ont comme fonction, la traduction d'éléments de connaissance et d'expériences quotidiennes liés au thème exploité et l'identification du statut de l'enfant comme acteur.

D'autres rôles initiés et suscités par la présence de personnages-figurines accessoires et organisés dans différents espaces de la production ont comme fonction: la relance du jeu par un thème nouveau et l'investissement de l'enfant comme acteur par l'intermédiaire de personnages-figurines. Quand les rôles initiés et joués par l'intermédiaire de personnages-figurines, sont des animaux ou des personnes, ils ont comme fonction: la traduction d'éléments de connaissance des enfants à propos du thème exploité au fur et à mesure de l'élaboration de la trame symbolique; l'investissement de l'enfant comme acteur par l'intermédiaire de personnages-figurines et la manifestation du chevauchement, de l'intégration et la complémentarité de l'intercoordination des rôles, reflétant la préoccupation de l'enfant à définir un rôle en fonction d'un autre rôle.

En outre, des rôles de caractère stéréotypé et de fiction sont initiés et joués au fur et à mesure du déroulement de la trame symbolique et traduisent des éléments de connaissance des enfants eu égard au thème exploité en lien avec la production et du rapport qu'ils établissent avec ces connaissances dans leur façon de le jouer. 
Finalement, des rôles non joués, émergeant d'une activité de jeu symbolique, peu complexes et peu élaborés, ont comme fonction la manifestation d'une préoccupation des éléments de connaissance des enfants sur l'intercoordination des rôles.

Tous ces rôles nommés, initiés et/ou joués émergent de l'activité de jeu symbolique et se construisent à partir des productions de l'activité de construction. Tous ces rôles sont en lien soit avec le projet global, soit avec les projets secondaires de cette activité de construction. Il est à souligner que seulement une séance propose des rôles de caractère stéréotypé et des rôles périphériques sans lien avec le projet global et les projets secondaires de l'activité de construction. Ces rôles sont initiés mais non joués et avaient comme fonction, l'exploration de divers possibles à la recherche d'une éventuelle trame symbolique à élaborer.

On peut aussi noter que cette même séance présente des rôles initiés à partir des éléments du discours des enfants de l'autre dyade, sans lien avec la trame amorcée. Ces rôles avaient pour but d'apporter des idées nouvelles dans l'activité de jeu symbolique.

\section{- L'adoption de rôles dans l'activité de jeu symbolique simultanée à l'activité de construction}

L'adoption de rôles dans les formes particulières d'agencement dans l'activité de construction et dans l'activité de jeu symbolique fait appel au même type de rôles et aux mêmes fonctions dégagées dans l'activité de jeu symbolique subordonnée à l'activité de jeu de construction. Cependant, la simultanéité épisodique de courte durée de l'activité de construction dans l'activité de jeu symbolique révèle que l'activité de jeu symbolique dominante engendre une quantité de rôles (39) tantôt nommés, tantôt initiés et aussi joués.

Ces rôles sont tous en lien avec le projet global et ont comme fonction: la traduction d'éléments de connaissance d'un ensemble de rôles susceptibles d'être joués dans le contexte du thème exploité à partir de l'activité de construction, la traduction de nombreux éléments d'expériences observés et/ou vécus par les enfants et finalement la manifestation d'un grand souci de complémentarité et d'intercoordination des rôles. 


\subsubsection{Synthèse des résultats relatifs aux composantes structurales dans l'organisation à quatre}

\section{- Les substitutions d'objets}

Les substitutions symboliques simples d'objets dans l'organisation à quatre se sont réalisées dans le contexte de l'activité de construction et d'autres réalisées à l'intérieur du scénario s'élaborent dans le contexte du jeu symbolique.

Les substitutions symboliques simples d'objets répondent spécifiquement à trois fonctions. La première de ces fonctions est l'attribution d'une signification particulière à un bloc dans le but d'une représentation matérielle de l'objet impliquant une transformation essentiellement symbolique et un partage de cette signification avec les partenaires.

La deuxième concerne l'attribution d'une signification particulière à un agencement de blocs (espace et forme) dans le but d'une représentation matérielle et spatiale d'un espace spécifique dans la production impliquant une transformation matérielle et symbolique et un partage de cette signification avec les partenaires.

Finalement, la troisième fonction est l'attribution d'une signification particulière à un agencement de blocs dans le but d'identifier un objet spécifique impliquant une transformation matérielle et symbolique et un partage de cette signification avec les partenaires.

Les substitutions symboliques à l'intérieur de l'activité de jeu symbolique sont toutes des transformations symboliques d'un bloc de bois. Elles sont ponctuelles et se réalisent au fur et à mesure du développement du thème; elles apparaissent en chapelet, fixent et relient les divers éléments du scénario.

\section{- L'adoption de rôles}

Dans ce jeu à quatre où très tôt l'activité de construction et l'activité de jeu symbolique se poursuivent simultanément de façon épisodique de courte et de longue durée, l'adoption de rôles sert différentes fonctions. Certains rôles fonctionnels périphériques, de caractère stéréotypé, en lien avec le projet global pendant l'activité de construction qui domine l'activité de jeu symbolique, posent des ancrages dans l'activité de construction 
susceptibles éventuellement d'être développés. Ils sont suscités par la production en cours (bancs de secours, système d'alarme), par des espaces, et entraînent certains gestes rattachés à ces espaces (lac-baigneur). D'autres rôles se définissent par des trames symboliques plus développées de l'activité de jeu symbolique.

L'analyse des composantes structurales du jeu symbolique apporte des éléments nouveaux dans la compréhension de la dynamique de l'enfant avec le matériel bloc. En effet, les composantes retenues ont surtout été observées et analysées dans le jeu de fiction dans les différentes études consultées. Cependant, le caractère particulier du matériel bloc engendre en premier lieu, une activité de construction. Dans cette première activité, l'enfant élabore des productions et crée ainsi un contexte. Comme l'enfant est engagé dans une activité de construction qui met en oeuvre la représentation, il utilise divers modes de substitutions d'objets qui remplissent différentes fonctions dépendant de la forme de participation sociale privilégiée. De plus, les composantes structurales, habituellement observées dans le jeu symbolique, se retrouvent également dans l'activité de construction. La présence de ces composantes permet de dégager des formes d'agencement impliquant des types de simultanéité qui amènent à constater que ces deux activités se chevauchent, s'interpellent et s'alimentent mutuellement provoquant des interactions sociales particulières, qui seront abordées dans la dernière partie de ce chapitre.

\subsection{Résultats relatifs à l'analyse des données de la troisième question de recherche}

La troisième question de recherche porte sur les interactions sociales dans la dynamique de l'activité de l'enfant avec le matériel bloc. Lors de l'analyse de cette activité, la communication verbale a été prise en compte puisqu'elle semble à la fois le fruit et la source de cette activité. De plus, l'ensemble des recherches recensées et examinées sur l'activité de construction avec le matériel bloc, met en évidence les produits de l'activité en termes de productions réalisées. Aucune de ces études n'a considéré la communication verbale comme élément constitutif, voire même structurant, de l'activité même de construction. Lors de l'analyse des séances d'observation, la communication verbale est apparue un élément marquant et dominant de cette activité autant dans les formes individuelles, associatives que coopératives de jeu. Cependant, la nature des énoncés dans la communication verbale se distingue selon les formes de participation sociale. 
De plus, l'activité de jeu symbolique s'est révélée une activité subordonnée ou simultanée à l'activité de construction selon des formes d'agencement impliquant des types particuliers de simultanéité. La communication verbale a également été prise en compte dans cette activité de jeu symbolique. Ainsi, la littérature examinée en rapport avec la recherche sur les interactions sociales et le jeu symbolique démontre depuis le début des années 80 , un intérêt majeur pour ce domaine. Les résultats de ces différentes recherches ont fourni des éléments de base à l'élaboration du cadre méthodologique d'analyse des interactions sociales dans l'activité de construction et l'activité de jeu symbolique.

Ainsi, dans l'analyse que nous avons privilégiée, l'ensemble des interactions sociales dans les formes de participation observées lors des séances de jeu, a été considéré afin de ressortir les stratégies interactives déployées par les enfants dans l'activité de construction et dans l'activité de jeu symbolique.

Par la suite, les négociations interpersonnelles ont fait l'objet d'une analyse plus approfondie puisqu'elles se manifestaient progressivement au cours de l'analyse, comme une forme particulière de communication présageant d'une possibilité de dénouement harmonieux et productif de l'activité et/ou d'une possibilité de rupture, et donc d'abandon complet ou d'une reprise de l'activité.

La synthèse des résultats relatifs aux interactions sociales s'est constituée à partir des trois phases distinctes de l'activité de l'enfant avec le matériel bloc. Le positionnement initial, l'élaboration de l'activité de construction et l'élaboration de l'activité de jeu symbolique ont été identifiés dès les premiers mouvements d'analyse.

\subsubsection{Synthèse des résultats relatifs aux interactions sociales dans la première phase: le positionnement initial}

Les résultats d'analyse de la première phase du positionnement initial, rappelons-le, montrent que les interactions sociales ont pour but la recherche d'un partenaire pour une éventuelle association. Ces interactions auraient comme objet de négociation les éléments de persuasion pour s'associer à des éventuels partenaires. Il faut noter que la durée de cette première phase est relativement courte, car la recherche d'un partenaire se réalise dans les cinq premières minutes de la séance et les enfants déploient un ensemble de stratégies interactives afin de convaincre et de persuader un autre enfant pour jouer ensemble. 
Ainsi, ils utilisent différentes façons pour initier une tentative d'association. Un enfant peut interpeller différents compagnons jusqu'à l'acceptation d'un de ces enfants à s'associer à son projet. Il peut interpeller un seul partenaire potentiel et à son refus, abandonner toute autre tentative, il peut accepter une proposition d'un autre enfant sans essayer aucune tentative d'association, ou, en dernière instance, il peut refuser toute proposition d'association.

D'autres stratégies interactives d'association étaient utilisées soit pour interpeller ou verbaliser le projet. Des stratégies d'offres d'aide, d'évaluations, d'attirances, de conseils, d'avertissements, de propositions, de demandes, d'invitations, de directives et d'annonces sont alors déployées par les enfants. Ils sont autant d'exemples éloquents pour établir une association avec un éventuel partenaire et chacun des enfants demeure libre d'accepter ou de refuser.

Dans cette même phase, les négociations de courte durée impliquant des stratégies d'entente peu élaborées entraînent dans la plupart des cas un déroulement représentant peu de moments conflictuels entre les partenaires intra-dyades et entre les membres interdyades. Cependant, les négociations de plus longue durée impliquant des stratégies plus élaborées et plus complexes dans les tentatives d'association et des refus explicites de non-association entraînent un dénouement représentant des moments conflictuels entre les membres inter-dyades.

\subsubsection{Synthèse des résultats relatifs aux interactions sociales dans la deuxième phase: l'élaboration de l'activité de construction}

Dans la phase d'élaboration de l'activité de construction, l'analyse des données permet d'affirmer que les interactions avaient pour but la réalisation partagée de l'intention du projet global et des projets secondaires. Elles avaient comme objet de négociation, les éléments de l'activité de construction nécessaires à l'agencement du matériel bloc afin de représenter la production envisagée.

Les stratégies interactives déployées par les enfants sont les mêmes quant à la forme, mais leur contenu se déplace sur les éléments de construction qui rendent possible l'élaboration partagée du projet global. Ces stratégies interactives se regroupent en quatre catégories: des interactions à propos de la réalisation du projet global et du ou des pro- 
jet(s) secondaire(s), des interactions à propos du matériel bloc, des interactions plus générales et des interactions sociales spécifiques. Celles-ci peuvent être analysées dans trois situations différentes: dans les séquences d'accord intra-dyade, dans les séquences de désaccord intra-dyade et, finalement, dans les événements de désaccord inter-dyade.

Les interactions sociales dans les séquences d'accord intra-dyade mettent en évidence des habiletés, des compétences et des stratégies qui favorisent un déroulement cohérent, harmonieux de plus en plus productif de l'activité en cours. Ces interactions s'élaborent à partir du respect et de l'écoute des idées, des propositions et du matériel, et permettent un dénouement intéressant de l'activité ainsi que le maintien de l'intérêt constant et soutenu pour jouer ensemble.

Elles exigent une élaboration de stratégies afin de conserver et de consolider l'association et ainsi contribuer à un dénouement intéressant et satisfaisant du jeu où chacun semble trouver son profit.

De plus, ces interactions sociales mettent en lumière une décentration de son point de vue et une sollicitation du point de vue de l'autre qui témoignent de l'apport de l'autre à la proposition initiale et du partage d'un même niveau de communication autant sur le plan du contenu même de l'activité de construction (idées, projet global, projet secondaire, matériel-accessoire, emplacement, transformations symboliques, transformations matérielles, etc.) que sur le plan de la qualité de la relation interpersonnelle.

Dans les séquences de désaccord intra-dyade, les interactions sociales mettent en évidence l'objet de négociation dès les premières répliques où origine le désaccord et portent sur des éléments explicites de l'activité de construction. Par exemple, sur le choix du projet secondaire et implicitement sur des ambiguïtés autour de l'entente à propos de celui qui assure le statut de meneur de jeu et celui qui assure le statut d'exécuteur.

L'analyse de ces séquences de désaccord nous amène à constater que le contenu (sens) explicite voile et masque ce qui semble constituer l'élément fondamental de l'ambiguïté dans la relation qui se trouve dans les rapports interpersonnels plutôt que dans le contenu de l'activité. 
Ainsi, la poursuite et la reprise de jeu semblent être possibles lorsqu'une partie ou la totalité de l'ambiguìté est explicitement mise à jour par différentes stratégies d'acceptation du statut de soi-même et de l'autre, des compétences de l'autre et de la reconnaissance de ses propres compétences.

L'analyse des interactions sociales dans les événements de désaccord interdyade présente des pistes de questionnements intéressantes. En effet, déjà dans la phase de positionnement initial, les interactions d'association donnent des résultats insatisfaisants pour quelques membres du groupe. Cette insatisfaction s'est manifestée par l'initiation de divers événements conflictuels, par la nature des événements initiés (statuts des membres, compétences, capacités, ingérence des actions des autres, évaluation négative de l'activité des autres, commentaires et mises en garde, répartition du matériel, droits et responsabilités), par la réaction vive de l'enfant à un événement qui semble réinvesti sur un plan personnel, et enfin par une insatisfaction qui semble se traduire par le même enfant, mais où cet enfant sert implicitement de bouc émissaire à une dyade qui en apparence semble bien fonctionner.

Ces interactions dévoilent aussi une certaine maturité et flexibilité des enfants à réinvestir des ambiguïtés possibles sur la relation en ne se laissant pas déstabiliser par la réplique émise et en réinvestissant sur le plan symbolique. Les enfants peuvent aussi élaborer des stratégies de chantage et de menaces pour mettre fin au désaccord, ou des stratégies de recadrage de la tâche en cours.

Nous avons également observé l'utilisation, dans une situation similaire de désaccord, des mêmes stratégies dont les résultats furent positifs, dans le but d'empêcher la dégénération de la situation et la rupture ainsi que la répétition et la reproduction de diverses situations qui produisent l'émergence du même objet de négociation. Ces situations semblent indiquer une problématique ou une ambiguiité plus profonde et non résolue que celle liée au contenu même de l'activité de construction.

En résumé, l'ensemble des événements inter-dyades laissent apparaître que l'objet central des négociations se situe soit sur la compétence de chacun des membres à réaliser la construction, soit sur les droits et les responsabilités en lien avec les concepteurs des productions ou soit sur les statuts de chacun, meneur/exécuteur, dans la dynamique de l'activité de construction. 


\subsubsection{Synthèse des résultats relatifs aux interactions sociales dans la troisième phase: l'élaboration de l'activité de jeu symbolique}

Les résultats d'analyse de cette troisième phase concernant l'activité de jeu symbolique montrent que les interactions sociales générées pendant cette activité avaient pour but l'élaboration partagée de scénarios et comme objet de négociation, les éléments de l'activité de jeu symbolique nécessaires à la création de scénarios élaborés par et autour des productions de l'activité de construction.

Les stratégies interactives déployées par les enfants sont les mêmes que celles de l'activité de construction quant à leur forme, mais leur contenu se déplace sur les éléments du jeu symbolique et rendent possible l'élaboration partagée du projet de jeu.

Concernant les éléments de l'objet de négociation, les stratégies interactives se présentent dans une première catégorie d'interactions qui assurent la transition entre la fin de l'activité de construction et le début de l'activité de jeu symbolique.

Par la suite, des interactions à propos d'éléments symboliques apparaissant dans l'activité de construction, sont des prémisses à d'éventuels scénarios dans l'activité de jeu symbolique ultérieure et de nombreuses interactions s'établissent à propos des éléments nécessaires à la mise en place du thème et à propos des éléments à l'intérieur du scénario, soit des éléments constitutifs de développement du scénario et à propos de la métacommunication.

Cependant, il faut noter la présence d'interactions sociales spécifiques dans les séquences d'accord intra-dyade, les séquences de désaccord intra-dyade, et dans les événements de désaccord inter-dyade.

Les interactions sociales dans les séquences d'accord intra-dyade témoignent d'habiletés, de compétences et de stratégies souvent très élaborées et complexes qui contribuent à un enchaînement cohérent, de plus en plus productif dans le déroulement de l'activité de jeu symbolique.

Ces interactions sont des indicateurs d'une bonne maturité ainsi que de décentration de son propre point de vue. Les différents partenaires proposent, ajoutent des spécificités 
au projet de l'autre, reprennent des idées à leur compte, modifient, contre-proposent, améliorent, acceptent, refusent, toujours dans le respect de l'autre et de ses propres idées et compétences. Ainsi, l'intérêt et le souci du «rester ensemble» semblent le point central des différentes stratégies négociatives que les enfants mettent en oeuvre.

Concernant les interactions sociales dans les séquences de désaccord intradyade, on remarque qu'elles provoquent une confrontation entre les enfants afin de les obliger à faire face à une situation problématique. De plus, ces interactions présentent à la fois des stratégies de résolution de problèmes qui échouent du fait que certaines phases du processus de résolution sont escamotées.

De ces interactions émergent également un certain nombre de questionnements: estce que les interactions des enfants les amènent à bien identifier la nature de leur problématique?; quelles sont donc leurs stratégies d'identification? et quelles sont les stratégies d'élucidation et les stratégies de résolution?

Les interactions sociales issues d'une situation représentative d'une séance d'observation a permis d'apprécier la nature et les éléments de base de certains épisodes conflictuels, ainsi que l'entraînement et le dénouement de ces moments conflictuels qui, dans certains cas, arrivent à un dénouement satisfaisant et dans d'autres cas, arrivent à une impasse sans déroulement satisfaisant. Par contre, dans les deux cas, les enfants apprennent progressivement à confronter des situations problématiques et à expérimenter différentes stratégies dans la résolution des conflits.

Ainsi, les stratégies de négociations déployées par les enfants dans leur tentative pour dénouer les ambiguïtés liées au contenu du jeu et à la relation interpersonnelle permettent déjà d'apprécier différentes réussites des enfants quant à leur façon d'apprivoiser graduellement les moments conflictuels.

Même si dans la séquence représentative des conflits qui peuvent survenir dans l'activité de l'enfant avec le matériel bloc, la durée de la séance n'a pas permis aux enfants d'entrer dans une phase de négociation productive, les enfants ont réussi:

- $\quad$ à poser des bases explicites de l'objet de négociation, donc à dénouer peu à peu, très lentement, des pistes d'ouverture sur la vraie nature du conflit; 
- $\quad$ à affronter progressivement, à petites doses, les vrais éléments de négociation quant au statut, aux droits, aux responsabilités de chacun dans la relation interpersonnelle;

- à formuler en termes clairs et explicites des répliques témoignant de leur position dans les ambiguités du conflit;

- à démontrer leur ténacité et leur courage à l'affrontement de situations conflictuelles par la recherche d'une multitude de stratégies pour démêler les ambiguités de la situation;

Ils ont aussi appris progressivement à risquer de confronter la situation problématique:

- par une prise de conscience graduelle des vrais éléments de l'objet de négociation qui parfois demeurent partiellement élucidés;

- par une persévérance dans son désir de jouer avec l'autre et par la recherche personnelle de moyens pour affronter la situation conflictuelle sans l'intervention d'un adulte;

- par leur capacité à faire face progressivement à leurs conflits internes, à les expliciter et à tenter de les maîtriser;

- par leur capacité à aborder et à trouver des pistes de résolution, voire même à résoudre des situations paradoxales qui restent toujours très difficiles, même chez les adultes.

Finalement, les enfants démontrent de façon admirable qu'ils possèdent spontanément beaucoup de ressources leur permettant de s'introduire peu à peu sur des pistes de négociations productives et que ce processus commence tôt dans la vie.

En effet, les observations ont montré que les enfants, même s'ils ne réussissent pas clairement à dissiper les ambiguïtés, sont capables de mettre en place un certain nombre de conditions essentielles pour parvenir à une négociation fructueuse par: 
- une ouverture à la négociation assurant la réciprocité de l'interdépendance; c'est une ouverture progressive qui, au début, est marquée par le positionnement de chacun sur son propre territoire en impliquant des stratégies de persuasion et de concession qui marquent de plus en plus les niveaux différents de communication;

- par une prise en compte et une prise de conscience graduelle des démêlés de l'ambiguiité en essayant de rendre explicite le véritable objet de négociation, afin d'éclaircir pour lui-même d'abord et pour l'autre, la vraie nature du conflit, route sinueuse, route tortueuse avec des retours en arrière sur des stratégies répétitives non fructueuses mais qui assurent une transition permettant d'affronter le conflit;

- par l'exploration d'alternatives plus novatrices de pistes pouvant mettre en évidence le problème de la situation afin que chacun détermine sa position (soi-même) et la position de l'autre;

- par l'utilisation de trois des quatre stratégies communicatives exprimant le niveau d'intégration moi-autrui identifiées par Bonica (1990).

La première stratégie est l'intégration moi-autrui: découverte et gestion des contraintes. Les exemples suivants démontrent cette stratégie. «Regarde, attends un peu, moi j'ai deux garages, toi t'as trois garages, toi t'as ces trois-là, moi j'ai ces deux-là, OK. Non, moi je prends ces deux-là, lui, pis lui, toi tu vas en avoir trois, lui, pis lui, pis lui.. Non, je prends lui, pis lui, toi tu prends lui, pis lui. OK je sors mes autos d'là, toi aussi. Moi j'en mets, toi, comment t'as d'autos?» «Ben là, ferme les portes. Ben là, tu l'installes de même. Prends tes autos que tu veux là, moi je l'arrange.»

La deuxième stratégie est l'utilisation de la structure temporelle. Voici un exemple: "Regarde là, on voit encore des autos. Comment tu fais pour y aller?»

Et finalement, la troisième stratégie concerne l'utilisation du moi et du nous dans un contexte spatial, par exemple: «Laisse là! Là! Là! Moi, moi je fais les escaliers, on va la placer de même, on va la mettre de même.» 
Ces différentes stratégies déployées par les enfants montrent leur capacité à construire une communication verbale entraînant une implication simultanée du moi et d'autrui. Par ces stratégies, ils développent progressivement leur alternance, leur positionnement et leur capacité de distanciation de soi et de l'autre dans une situation spécifique de jeu.

Les interactions sociales dans les événements de désaccord inter-dyade démontrent que l'ensemble des répliques constituant les événements de désaccord ont comme objet de négociation les responsabilités, les droits de chacun dans leur dyade et ils se posent très explicitement sur le plan de la réalité lors des discussions et des échanges. Certaines répliques sont des affrontements sur le plan personnel qui ne mènent nulle part, sauf à dévaloriser autrui, et les incursions et les infiltrations d'une tierce personne, pair, sans préavis, sur des décisions du déroulement de la structure du jeu ne font qu'envenimer dans cette séance la situation inter-dyade. Finalement, une intervention sollicitée de l'enseignante pour la résolution de leur conflit permet de dégager des jalons possibles d'interventions pédagogiques:

- le rétablissement des faits de la situation conflictuelle à partir des versions de chaque membre impliqué, afin de rendre explicite la source du conflit et de poser les bases d'une résolution éventuelle impliquant une attitude d'ouverture de l'enseignante;

- l'identification du rôle de l'enseignante à partir de la demande sollicitée des enfants. L'enseignante doit d'abord et avant tout préciser son rôle à partir de la demande des enfants. De quel type d'aide ont-ils besoin? À quoi s'attendent les enfants par leur sollicitation de l'enseignante: un rôle de médiateur, d'aide, d'une personne adulte extérieure pour régler le conflit?

- l'importance de savoir questionner. Une deuxième attitude d'ouverture se situe sur le plan du questionnement:

- questions de recontextualisation des événements de la situation;

- questions pour l'expression des sentiments et des émotions liés à la situation;

- donner du temps à chacun afin d'établir la problématique de la situation et de s'exprimer sur le plan émotionnel; 
- l'exploration de la recherche de solutions par les enfants, par les enfants et l'adulte.

\section{4 Émergence d'une compréhension et l'ouverture de nouveaux re- gards pour la recherche et l'intervention pédagogique}

Les différentes synthèses de l'analyse des données eu égard aux questions de recherche ont tenté de dégager la dynamique globale et interne de l'activité que le matériel bloc suscite chez l'enfant. Pour mettre en lumière les apports de ces différentes analyses dans la compréhension des potentialités du matériel, des composantes structurales et des produits de l'activité de l'enfant avec le matériel bloc et de la nature et de la structure de cette activité, rappelons la trame de questionnement de notre problématique de recherche.

Considérant les deux questions générales de recherche: Quelle est la nature des activités que le matériel bloc suscite chez l'enfant ? Est-ce que ce matériel et les activités qu'il suscite, contribuent au développement cognitif et social de l'enfant qui fréquente la classe maternelle? Celles-ci nous ont amenée à formuler trois questions spécifiques de recherche selon trois enchaînements successifs qui témoignent de la dynamique que l'on désirait observer, décrire, analyser et comprendre.

Du premier enchaînement, dans la compréhension de la nature de l'activité comme étant d'abord une activité de construction, nous avons identifié certains paramètres de construction correspondant à des stades ou étapes d'évolution de l'activité de construction, d'où la première question spécifique de recherche: À quel stade ou à quelle étape d'évolution de l'activité de construction se situent les enfants de cinq-six ans qui fréquentent la classe maternelle?

Le deuxième enchaînement s'inscrit également dans la compréhension de la nature de l'activité de l'enfant comme étant, par la suite, une activité de jeu symbolique, à partir et autour des différentes productions réalisées dans l'activité de construction. Ce deuxième enchaînement veut mettre en évidence par l'observation, la description, l'analyse et la compréhension des spécificités de l'activité de jeu symbolique élaborées à partir du matériel bloc. Ainsi se formule la deuxième question de recherche: Quelles sont les composantes structurales de l'activité de jeu symbolique avec ce matériel? 
Enfin, le troisième enchaînement s'insère dans la compréhension des interactions sociales qui sous-tendent l'activité de construction et l'activité de jeu symbolique. Cet enchaînement vise à dégager un ensemble d'éléments essentiels dans la structure de l'activité de l'enfant avec le matériel bloc se situant sur le plan interactionnel, soit les interactions sociales entre les enfants. Ces éléments influenceraient et détermineraient l'activité de construction et l'activité de jeu symbolique simultanée ou subordonnée à l'activité de construction.

D'où la troisième question de recherche: Quels types d'interactions sociales émergent de l'activité de l'enfant avec le matériel bloc? et, est-ce qu'elles contribuent au développement cognitif et social de l'enfant? Quelles sont les interactions sociales qui émergent de la dynamique de l'activité de construction au coin bloc ? Quelles sont celles qui favorisent davantage la réalisation d'un projet collectif de construction? Quelles sont les interactions sociales qui émergent de la dynamique de l'activité de jeu symbolique au coin bloc ? Quelles sont celles qui favorisent davantage la réalisation d'un projet collectif de jeu symbolique?

La synthèse relative à l'analyse des données de la première question de recherche permet de confirmer que les enfants observés manifestent dans leur activité de construction avec les blocs de bois vernis, un développement se situant aux stades cinq et six tel qu'observé dans les travaux de Johnson (1974). Cependant, il faut apporter certaines nuances à ces résultats puisque plusieurs éléments nouveaux ont été pris en compte dans notre recherche.

En effet, sur le plan des habiletés et des compétences techniques, nos observations se sont réalisées à partir des produits de construction des enfants, mais ces habiletés et ces compétences ont été observées pendant toute la durée de l'activité de l'enfant. Ainsi, les composantes de l'activité de construction ont été observées à partir d'éléments indicateurs nouveaux tels que les productions annoncées et les changements en cours d'activité, le temps, les moments distincts de la répartition du temps pour l'activité de construction, l'utilisation d'accessoires, l'enfant acteur ou utilisateur de personnages miniatures, les types de représentations symboliques et les formes de participation sociale. Ainsi, nous avons élargi le filtre d'observation afin de considérer les composantes, les produits, le temps et les diverses formes de participation sociale. Certains de ces éléments considérés dans d'autres études permettent de réaliser dans une même recherche l'observation et l'a- 
nalyse de tous ces éléments. Nous posons donc un regard beaucoup plus global et complet et apportons la perspective d'ensemble d'un grand nombre d'éléments dans la compréhension de l'activité de l'enfant avec le matériel bloc, ce qui supporte le choix et la pertinence de nos trois questions de recherche dans la même étude.

Sur le plan des compétences communicationnelles eu égard de l'activité de construction, la prise en compte de la communication verbale est un élément tout à fait nouveau dans la compréhension de l'activité de l'enfant avec le matériel bloc.

En effet, de toutes les recherches sur le matériel bloc, notre étude est la seule à considérer l'apport de la communication verbale dans cette dynamique de jeu. Cette prise en compte de cette communication permet de dégager des habiletés et des compétences communicationnelles non considérées jusqu'à maintenant dans cette activité de construction. Ce nouvel élément permet donc d'élargir la compréhension de cette dynamique de jeu au-delà du matériel lui-même. Il établit l'apport significatif de l'enfant dans son rapport au matériel lui-même et de l'apport de l'autre, dans cette activité. Les différents énoncés verbaux des enfants dans l'activité de construction et ses modes d'organisation soit solitaire, parallèle, associatif et coopératif mettent en lumière les intentions et les verbalisations des enfants dans son activité de construction.

Sur le plan des produits de l'activité, les différentes tâches identifiées par Cuffaro (1984) ont permis de recadrer l'ensemble des productions des enfants et des rapports qu'ils établissent dans la création d'un contexte de base, dans la transposition de leurs idées sur le plan opérationnel par l'intermédiaire des blocs et dans la distanciation de "soimême", comme acteur et scénariste. La variété des productions susceptibles d'être réalisées à l'aire des blocs fait ressortir les nombreux intérêts et les différentes préoccupations des enfants. De plus, l'analyse des données a permis d'identifier un ensemble de projets globaux, qui sont des projets pivots constituant le contexte de base de l'activité de construction. Ils regroupent quatre grandes catégories de productions et un ensemble de projets secondaires et complémentaires se réalisant selon des modalités de transformations matérielles et symboliques. De plus, l'analyse a mis en évidence l'élaboration de deux types de structure. Une première s'élaborant à la dimension de la taille des enfants et une deuxième à la dimension de la taille des figurines. L'élaboration de ces structures entraîne donc une implication et un investissement très différents de la part de l'enfant dans l'activité de construction. 
Sur le plan du temps consacré à l'activité, l'analyse de la répartition de ce temps a dégagé le temps d'investissement de l'enfant pour l'activité de construction et pour l'activité de jeu symbolique. D'où l'importance de maintenir l'accessibilité de ce matériel toute l'année en classe maternelle et de donner une durée assez longue à ce jeu dans la programmation quotidienne.

Finalement, sur le plan des diverses formes de participation sociale, nous avons observé un mode d'organisation à prédominance dyadique et solitaire, et un seul mode d'organisation à quatre pour l'activité de construction et de jeu symbolique. Étant donné l'âge des enfants (5-6 ans), nous nous serions attendue à l'observation de plus de formes coopératives d'organisation à quatre d'autant plus que les études d'un groupe de chercheurs (Baillon, Bonica, Bréauté, Mayer, Mussati, Rayna, Sinclair, Stamback, Verba, 1990) avait démontré que déjà au cours de la deuxième année, les enfants témoignent dans leurs échanges dans diverses situations de jeu, des capacités de coordination interpersonnelles et qu'à 3 ans, ils sont déjà en train de construire l'échange équilibré de pensée dont Piaget souligne l'importance dans le développement cognitif; qu'ils parviennent déjà à la conservation de thèmes sur lesquels ils se sont mis d'accord et qu'ils arrivent à l'acceptation de la réciprocité des propositions souvent à la suite de négociations et de recherche de compromis explicites, capacités généralement reconnues par Piaget sur le plan du raisonnement vers l'âge de 6-7 ans.

Il était donc opportun d'envisager que les interactions sociales des enfants de 5-6 ans soient plus développées ou du moins présentées des éléments de structure plus développées ou plus riches, témoignant de compétences cognitives et sociales plus élaborées facilitant ou contribuant de façon significative à la réussite d'une activité de construction et une activité de jeu symbolique collective, lesquelles se traduiraient par un répertoire de stratégies interactives plus évoluées permettant des types de négociations, d'ajustements, d'adaptation plus ou mieux consolidées et exploitées.

Les résultats obtenus tracent des pistes intéressantes d'investigation des formes coopératives à trois et quatre enfants qui ne sont pas sans interroger les compétences cognitives et sociales des enfants de 5 ans sur le plan des interactions sociales entre plusieurs enfants pour la réussite d'une activité de construction et d'une activité de jeu symbolique au coin bloc à la maternelle. 
L'ensemble de ces analyses constitue donc des données nouvelles quant à l'activité de l'enfant avec le matériel bloc et quant à sa compréhension.

Pour ce qui est des résultats de la synthèse relative à l'analyse des données de la deuxième question de recherche, celle-ci voulait mettre en évidence la préoccupation de l'activité de jeu symbolique suscitée par l'activité de construction. Cette analyse a permis de dégager un ensemble de composantes structurales spécifiques à l'activité de jeu symbolique mais aussi spécifiques à l'activité de construction. Les composantes structurales retenues à partir de celles du jeu symbolique identifiées par de nombreux auteurs ont mis en lumière des spécificités inhérentes aux substitutions d'objets, aux rôles et à l'intercoordination de ces rôles dans les différentes formes de participation sociale, solitaire, dyadique et à quatre, et ce, dans l'activité de construction et dans l'activité de jeu symbolique simultanée ou subordonnée à l'activité de construction. De plus, cette analyse a fait ressortir trois formes d'agencement et deux types de simultanéité dans ces deux activités et que, dépendant des formes de participation sociale de ces activités, les substitutions d'objets répondent à différentes fonctions. Ainsi, la structure interne de ces activités et les composantes structurales sont spécifiques et reliées à la matérialité même des blocs et, par la suite, aux productions engendrées par l'activité de construction et enfin, à l'activité de jeu symbolique subordonnée ou simultanée à l'activité de construction.

L'ensemble de ces résultats apporte des données tout à fait nouvelles eu égard aux composantes structurales de ces deux activités et vient définir un certain nombre d'éléments quant au type de jeu symbolique à partir et autour des productions. C'est une activité de jeu symbolique singulière et particulière en fonction des produits générés par l'activité de construction.

Finalement, la troisième question de recherche concerne les interactions sociales qui sous-tendent l'activité de construction et l'activité de jeu symbolique. C'est la prise en compte de la communication verbale qui a permis d'apprécier l'importance des énoncés verbaux des enfants comme étant à la fois le fruit et la source de cette activité de construction et de cette activité de jeu symbolique. La communication verbale est apparue comme un élément dominant et structurant autant dans les formes de participation individuelle et collective de jeu, un élément dévoilant tout un éventail d'informations sur le contenu et la forme de ces deux activités. L'analyse des données a fait ressortir un ensemble de stratégies interactives déployées par les enfants dans ces activités. Les négociations interper- 
sonnelles ont fait l'objet d'une analyse plus approfondie puisqu'elles présentaient une forme particulière d'interactions sociales essentielles et fondamentales dans la viabilité d'un projet partagé de construction et de jeu symbolique. Les résultats s'inscrivent dans la même lignée que ceux obtenus par Bonica (1990) dans l'observation et l'analyse de ces négociations dans le jeu de fiction des enfants, mais ils sont un apport significatif de l'activité de l'enfant avec le matériel bloc. Ils témoignent de la capacité des enfants à affronter et confronter des situations conflictuelles, à considérer progressivement le point de vue de l'autre et ces stratégies comme une source éventuelle d'apprentissage et de développement afin d'explorer des alternatives et des pistes novatrices à la résolution des conflits.

Ce regard intégré de l'activité de construction et du jeu symbolique devient une richesse pour l'organisation matérielle de cet espace de jeu en classe maternelle et amorce une piste importante pour l'intervention de l'enseignante auprès de l'enfant qui joue avec ce matériel.

L'ensemble de ces données vient donc élargir le champ de connaissances quant aux stades d'évolution de l'activité de construction, quant aux différentes composantes structurales et aux différents produits des activités de construction et de jeu symbolique et, finalement, quant aux interactions sociales qui influencent, déterminent et structurent à la fois le fruit et la source de ces deux activités. De plus, ces nouvelles connaissances qui émergent d'un filtre d'observation et d'interprétation constructiviste et socioconstructiviste viennent raffiner, compléter et relancer de nouveaux regards sur l'activité de l'enfant avec le matériel bloc afin de comprendre un peu plus et un peu mieux toute la richesse et la complexité de la dynamique de cette activité.

Dans le même ordre d'idée, cet ensemble de données nouvelles vient s'inscrire sur les plans technique, communicationnel, structurel et interactionnel dans la compréhension de l'activité de l'enfant et identifie de nombreuses habiletés et compétences des enfants contribuant ainsi à son développement cognitif et social.

La problématique de recherche, la démarche théorique et analytique ainsi que les résultats d'analyses viennent donc rejoindre les rangs des diverses préoccupations des chercheurs dans le domaine du jeu et du jouet. Toute cette démarche participe à l'ouverture d'horizons nouveaux quant aux pistes d'observation, d'interprétation et de compréhension de la dynamique de l'activité de l'enfant avec le matériel bloc. 
En ce sens, nous croyons que la portée de cette recherche présente un triple intérêt: théorique, méthodologique et pédagogique.

Théorique, parce que cette recherche vise à raffiner une problématique d'un contexte de jeu, où le matériel qui le sous-tend est, à la fois, générateur de l'activité et où l'activité devient à son tour, génératrice d'activités et d'interactions sociales. Il est donc important pour les chercheurs de redéfinir la problématique des activités de l'enfant avec différents types de matériel en général, et du matériel bloc en particulier, leur nature, leur structure, leurs composantes et leurs produits en partant d'un cadre référentiel dont l'axe fondamental repose sur les rapports entre les éléments théoriques, le développement cognitif et social, et leur impact sur les interventions pédagogiques. De ces données émergent donc de nouveaux éléments théoriques qui permettent, d'une part, de mieux comprendre l'activité de l'enfant avec le matériel bloc et de soutenir la pertinence de ce matériel et de cette activité à la maternelle et ce, à la lumière du nouveau contexte de la maternelle à temps plein dans les écoles du Québec. Ce matériel est considéré comme étant susceptible de contribuer à l'activité de jeu symbolique d'une façon tout à fait originale ainsi qu'au développement des interactions sociales en général, et aux négociations interpersonnelles en particulier, et d'apporter ainsi une contribution significative au développement cognitif et social de l'enfant.

Sur le plan méthodologique, cette étude, en fonction des fondements épistémologiques et théoriques retenus, a exigé l'élaboration d'une technique d'observation dans une approche qualitative de recherche impliquant une construction de la démarche et des cadres référentiels de mise en forme et d'analyse, tant sur le plan de la cueillette des données que de leurs analyses, puisant ses principes de base à la fois dans des techniques d'observation systématique et expérientielle. Sur ce plan, plus d'un défi a tenté d'être relevé. Nous croyons que l'une des originalités de cette étude se situe davantage sur ce plan, en considération des exigences reliées à l'élaboration de cette démarche méthodologique et analytique des données.

Finalement, sur le plan des interventions pédagogiques, nous croyons que ces données apporteront un renouveau et une reconnaissance du matériel bloc et des activités qu'il suscite auprès des intervenantes en classe maternelle et ce, sur plus d'un aspect. 
Un premier aspect concerne les modes d'intervention privilégiés de l'enseignante. En effet, l'enseignante, consciente du potentiel et de la pertinence de ce matériel et des activités qu'il suscite eu égard au développement cognitif et social de l'enfant, est un médiateur crucial entre le matériel et l'enfant pour soutenir l'intérêt des enfants face à ce matériel et pour les amener à progresser. Elle est appelée à intervenir de différentes façons.

L'intervention directe de l'enseignante permet d'influencer directement le choix de l'enfant et sa façon d'utiliser le matériel choisi. Cette intervention directe peut être, dans un premier temps, de montrer à l'enfant comment utiliser ce matériel et dans un deuxième temps, cette intervention peut être verbale pour expliquer, pour commenter, pour encourager et pour relancer le jeu.

L'intervention indirecte pour sa part, influence l'action de l'enfant par l'utilisation d'un intermédiaire entre lui et l'adulte. Au plan physique, cette intervention est l'environnement. La planification, l'organisation de l'aire des blocs avec un matériel en quantité suffisante et avec l'accessibilité d'un matériel-accessoire varié permettant d'enrichir, d'alimenter et de soutenir l'élaboration de projets globaux et secondaires manifestant les intérêts, les préoccupations, les connaissances et les expériences des enfants de cinq-six ans, influencent les choix et les comportements de jeu des enfants.

Au plan verbal, cette influence se manifeste par les paroles de l'enseignante qui ont pour but, non pas d'apporter des réponses et des solutions aux problèmes rencontrés par l'enfant, mais de l'amener à réfléchir, à progresser, à aller plus loin dans son jeu.

L'intervention indirecte se manifeste donc à travers l'aménagement de l'espace de l'aire des blocs, par le matériel de jeu disponible et accessible dans cette aire de jeu et par l'utilisation d'une intervention verbale qui conduit l'enfant à penser, à réfléchir, à se questionner, à prendre conscience, à prendre des risques et à trouver ses propres solutions aux problèmes soulevés soit par le matériel, soit par l'activité de construction, soit par l'activité de jeu symbolique et soit par les interactions sociales qui sous-tendent ces activités.

L'efficacité de ce type d'intervention demande des habiletés, des compétences, de la sensibilité, de l'ouverture et du temps consacré à l'observation à l'activité de construction 
et à l'activité de jeu symbolique. L'observation, comme le démontre notre recherche, met en évidence les produits, les différentes composantes des activités ainsi que les modes d'organisation de ces activités et des interactions sociales sous-jacentes qui deviennent des révélateurs du développement des enfants et des indicateurs du type d'aide et de soutien dont l'enfant peut avoir besoin pour progresser. De plus, l'observation de la dynamique sociale des enfants dans l'aire des blocs permet à l'enseignante de se sensibiliser à l'importance du rôle de l'autre et de la position de chacun des partenaires de jeu. Qui est le meneur?, Quelles sont les tâches, les rôles?, Quels types de stratégies interactives utilisent-ils pour " jouer ensemble " et assurer la viabilité du projet collectif de construction et de jeu symbolique. Tous ces questionnements amènent l'enseignante à se positionner sur le type d'intervention à privilégier.

Un deuxième aspect concerne plus spécifiquement le mode de fonctionnement par atelier privilégié en classe maternelle. Les données de cette recherche interrogent les principes de base de ce mode de fonctionnement quant aux diverses possibilités d'intégration des projets et des thèmes proposés aux enfants et de l'importance et de la richesse d'opérationnaliser l'exploitation de ces projets et de ces thèmes dans les différents ateliers et en particulier dans l'aire des blocs. Celle-ci pourrait ainsi devenir le berceau de nouveaux projets globaux et secondaires de l'activité de construction et de nouveaux scénarios de l'activité de jeu symbolique, en ouvrant ainsi les portes à de nouvelles connaissances et expériences afin de contribuer à élargir le bagage de connaissances et d'expériences des enfants sur des sujets variés qu'ils pourraient réinvestir dans l'activité de construction et de jeu symbolique.

Finalement, un troisième aspect se rapporte aux interactions sociales. Les résultats mettent en évidence l'importance de fournir aux enfants des environnements de jeu variés, où ils seront sollicités sur le plan personnel et interpersonnel et où ils pourront appréhender, apprivoiser et se confronter à des situations conflictuelles, et où ils auront la possibilité d'expérimenter différentes stratégies interactives pour préserver l'association et la viabilité de leur jeu. L'aire des blocs en classe maternelle est particulièrement féconde et fructueuse à l'égard des interactions sociales.

Si cette étude fait ressortir sur le plan théorique, de nouvelles connaissances pour mieux comprendre la dynamique des activités de l'enfant avec le matériel bloc, il importe de souligner les différentes limites soulevées tout au long de la démarche. 
Une première limite est liée au cadre restreint d'échantillonnage retenu. En effet, ce cadre peut être considéré comme une étude de cas, puisque la cueillette des données s'est réalisée dans une seule classe maternelle de la région 02 du Québec, composée de 19 enfants, agés entre cinq et six ans. Il serait intéressant de confronter les résultats d'analyse avec d'autres groupes d'enfants du même âge et ce, dans plusieurs classes maternelles pour apprécier une complexité plus globale des activités de l'enfant avec le matériel bloc et ainsi, élargir davantage les connaissances quant aux stades d'évolution de construction, aux composantes structurales, aux produits, à l'investissement du temps de réalisation des activités, aux divers modes d'organisation sociale et enfin, aux interactions sociales découlant de cette activité de construction et de jeu symbolique.

Une deuxième limite se situe sur le plan du caractère même d'une recherche de type qualitatif. En effet, dans une recherche qualitative, la nécessité d'embrasser globalement notre objet d'étude dans un but de compréhension de cet objet, oblige le chercheur à élaborer des cadres sophistiqués et complexes de mise en forme et d'analyse d'une "montagne" de données et d'informations provenant de diverses sources. Ce qui implique que le chercheur, tout au long de la démarche, doit effectuer des choix. Par ces choix, il est bien conscient de ne pouvoir tenir compte de toutes les possiblités d'analyses susceptibles d'éclairer la compréhension de son objet d'étude. Ainsi, il demeure que l'objet d'étude investigué embrasse une réalité construite qu'à partir des éléments privilégiés pour certaines analyses.

Ce type de recherche nous a amené à interroger la démarche solitaire du chercheur dans la construction de la compréhension de son objet de recherche à une époque où l'importance et la reconnaissance des confrontations interindividuelles sont loin d'être négligeables. À notre sens, il importe de repenser et d'élaborer des pistes et des cadres novateurs et créateurs dans la production des connaissances à deux ou plusieurs dans une démarche doctorale.

Une dernière limite nous est apparue dès le début de cette démarche doctorale, soit des limites sous-jacentes au processus d'écriture d'une thèse de type qualitatif. En effet, ce type de recherche s'est infiltré graduellement dans la communauté scientifique et certains chercheurs ont réfléchi sur le processus d'écriture d'une telle démarche. Cependant, encore aujourd'hui, étant donné la mince crédibilité accordée à ce type d'approche et aux différents outils disponibles pour rendre compte de l'écriture d'une thèse qualitative, les 
chercheurs se retrouvent, très souvent, dans des positions d'argumentation et de justification afin de construire la légitimité de ces approches qualitatives au sein de la communauté scientifique. Par contre, ces différentes positions présentent en elles-mêmes un certain avantage pour le chercheur. Il permet la création constructive de sa propre démarche et les risques encourus sont bénéfiques au plan de la création personnelle du processus d'écriture. Le désavantage se situe plus spécifiquement sur les consensus de la communauté scientifique pour rendre compte des normes et des cadres d'écriture encore teintés d'une démarche plus traditionnelle de recherche. La résistance au changement n'échappe pas au domaine de la recherche, sans toutefois, empêcher des tentatives différentes de ce processus d'écriture. La sensibilité à ce processus d'écriture témoignant de notre propre processus afin de rendre compte de notre démarche, nous a accompagnée et interrogée tout au long de la rédaction de cette thèse. 


\section{APPENDICE A}

A.1 Blocs de construction en bois vernis de Caroline Pratt.

A.2 Identification des différents stades ou étapes d'évolution dans l'activité de construction avec le matériel bloc selon Harriet Johnson (1974), Mary Ann Santa (1980), Maria Apelman (1984), Diane Dodge (1979) et Stuart Reifel (1984).

A.3 Ensemble de projets globaux et secondaires réalisés dans les neuf séances d'observation.

A.4 Identification de l'ensemble des rôles nommés, initiés et joués dans l'activité de jeu symbolique subordonnée à l'activité de construction, selon la classification de Garvey (1977). 


\section{A.1 Blocs de construction en bois vernis de Caroline Pratt}

Les blocs de Caroline Pratt sont des pièces de bois naturel vernis en érable. Cet ensemble de blocs est composé d'une variété de formes: quadrangle, cylindre, rampe, triangle, courbe elliptique, etc.). Pour réaliser des productions, l'enfant utilise la technique de superposition et de juxtaposition.

À partir de l'unité de base du demi-module et d'autres pièces de différentes formes, ce matériel offre à l'enfant l'occasion d'exprimer des besoins d'action, sa connaissance et son rapport au monde qui l'entoure. Ces blocs fournissent à l'enfant une infinie de possibles dans la création de productions dites ouvertes et figuratives. Avec ces blocs, l'enfant crée différents environnements tant imaginaires qu'inspirés du quotidien. Ces créations favorisent la mise en scène de situations personnelles et familières. De plus, étant donné la taille des blocs, les productions favorisent l'investissement de l'enfant lui-même comme principal acteur dans ces environnements, sans intermédiaire de figurines. Cet investissement apporte au jeu un autre niveau de connaissances et de mises en scène. 


\section{A.1 Blocs de construction en bois vernis de Caroline Pratt}

Série de blocs

Demi-module (1)

Module (2)

Double module (3)

Quadrangle (4)

Colonne double (non illustrée)

Colonne (5)

Petit cylindre (6)

Grand cylindre (7)

Arche quart de cercle (8)

Courbe elliptique (9)

Quart de cercle (non illustré)

Petit triangle (10)

Grand triangle (11)

Planche de toit (12)

Planche de grande construction (non illustrée)

Rampe (13)

Chaussée (145)

Y connecteur (15)

Arche (non illustrée)

Demi-cercle (non illustré)

Demi-arche romaine (non illustrée)

Grande connexion (non illustrée)

Porte gothique (non illustrée)

Carrefour (non illustré)

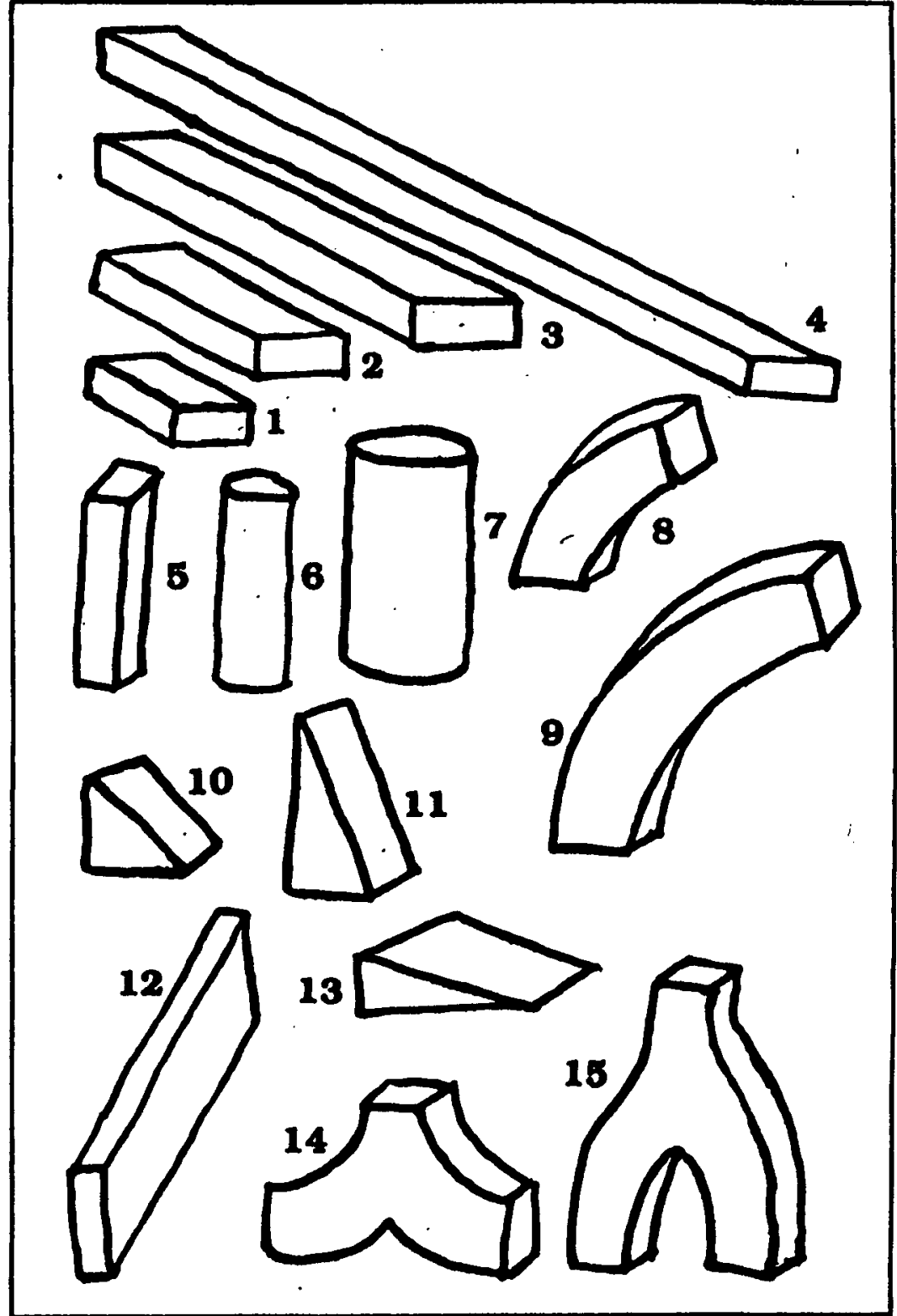


A.2 Identification des différents stades ou étapes d'évolution dans l'activité de construction avec le matériel bloc selon Harriet Johnson (1974), Mary Ann Santa (1980), Maria Apelman (1984), Diane Dodge (1979) et Stuart Reifel (1984)

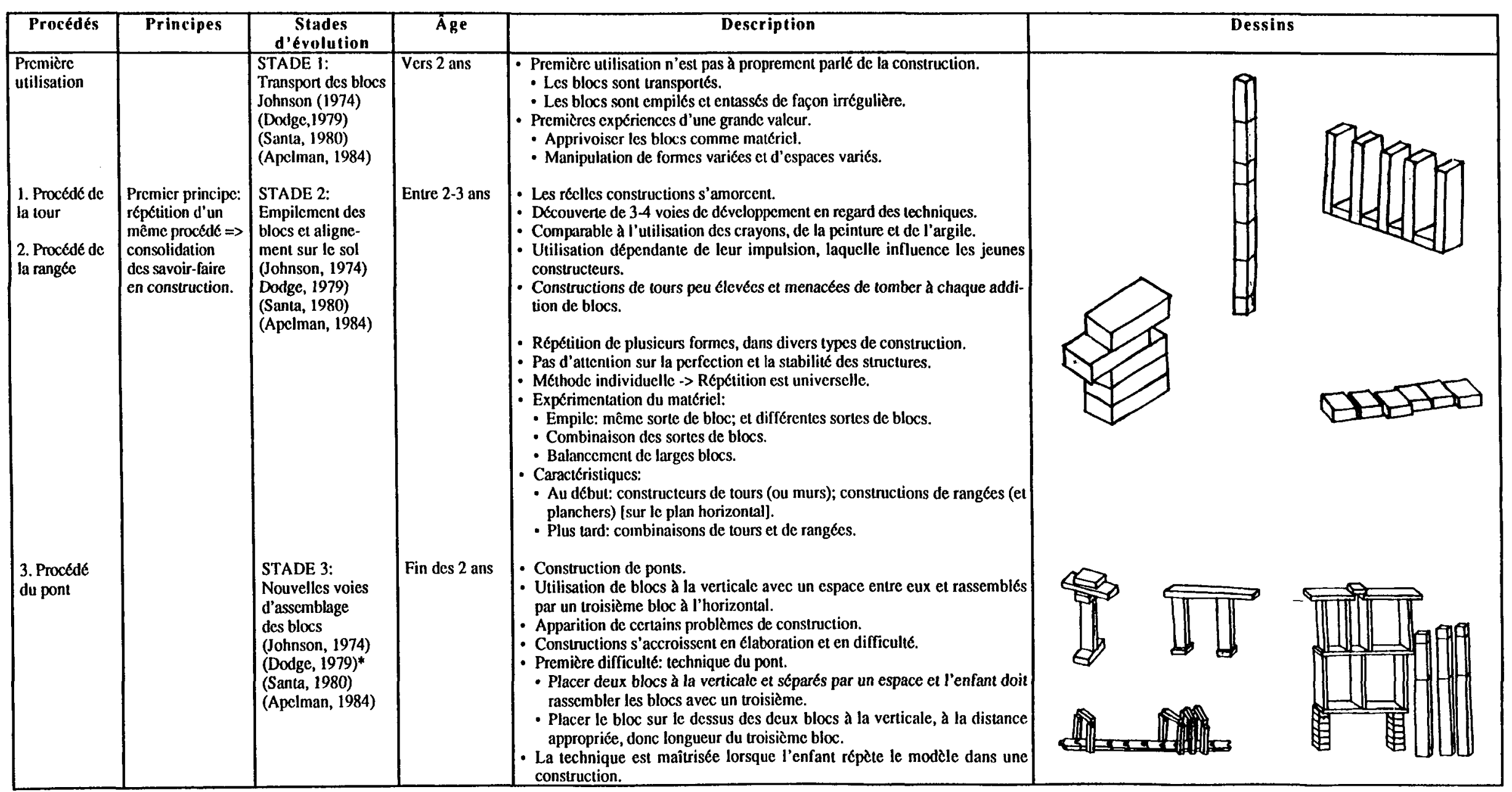

* Harriet Johnson (1974) identifie six stades d'évolution dans la construction.

* Diane Dodge (1979) propose quatre stades d'évolution de la construction inspirés des travaux de Johnson (stades 1, 2, 3, 4). 


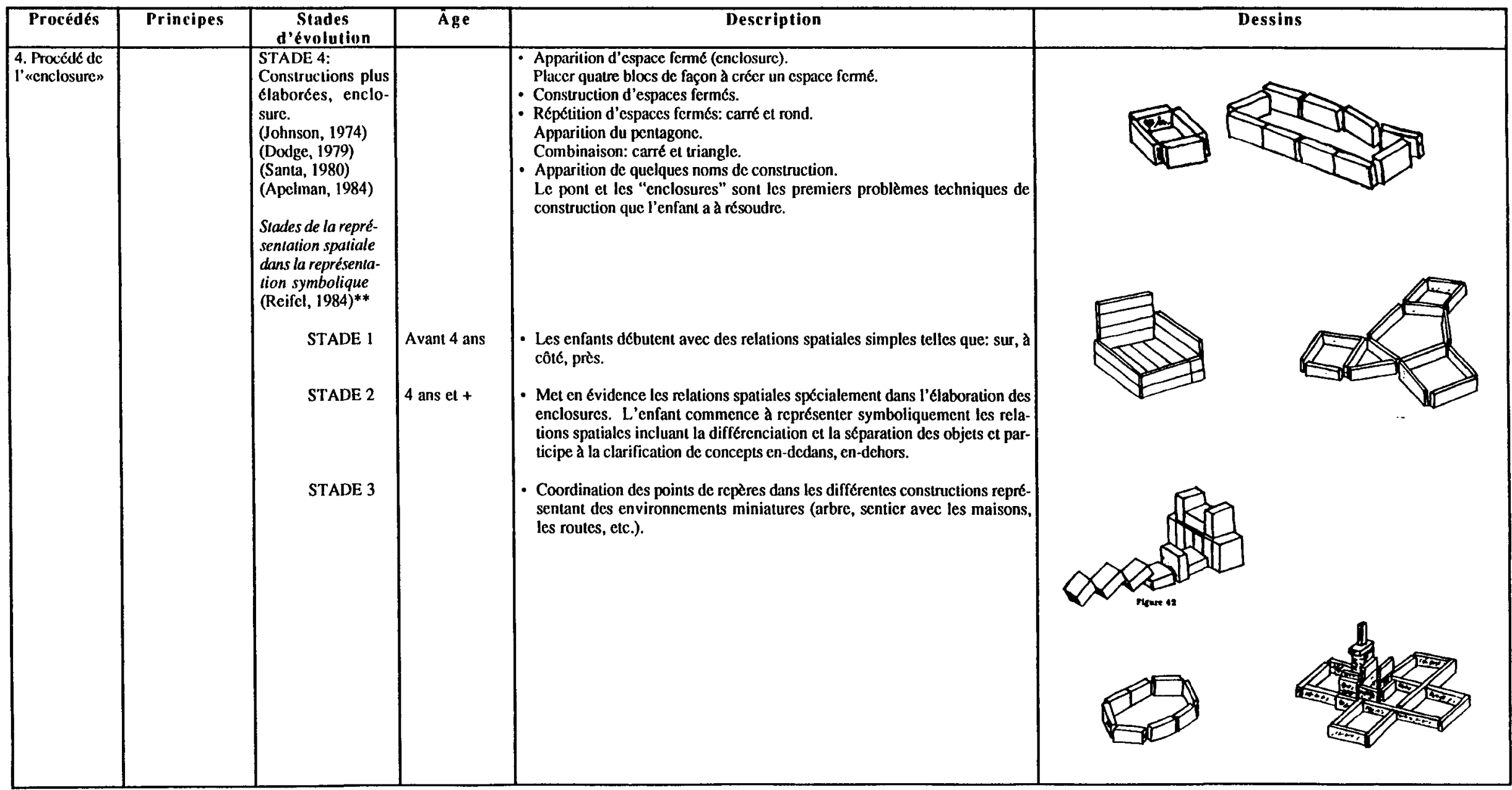

** Stuart Reifel (1984), inspiré des travaux de Johnson, identifie les différentes notions et relations spatiales qui peuvent être mises à contribution dans chaque procédé uuilisé par les enfants pour la réalisation de différentes productions. 


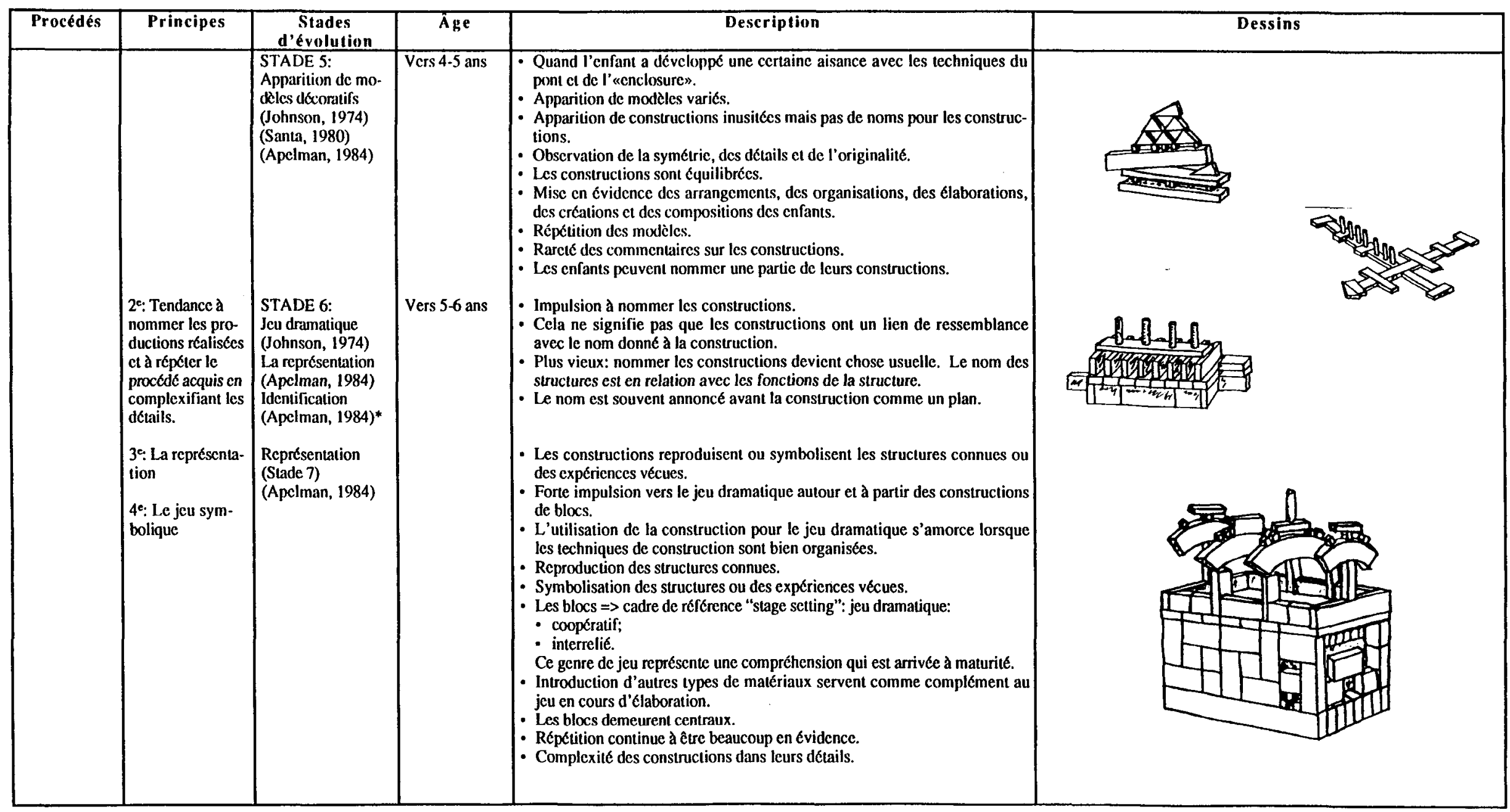

*Pour Maja Apelman (1984), le stade (6) de la représentation est divisé en deux élapes d'évolution distinctes: l'identification et la représentation. 


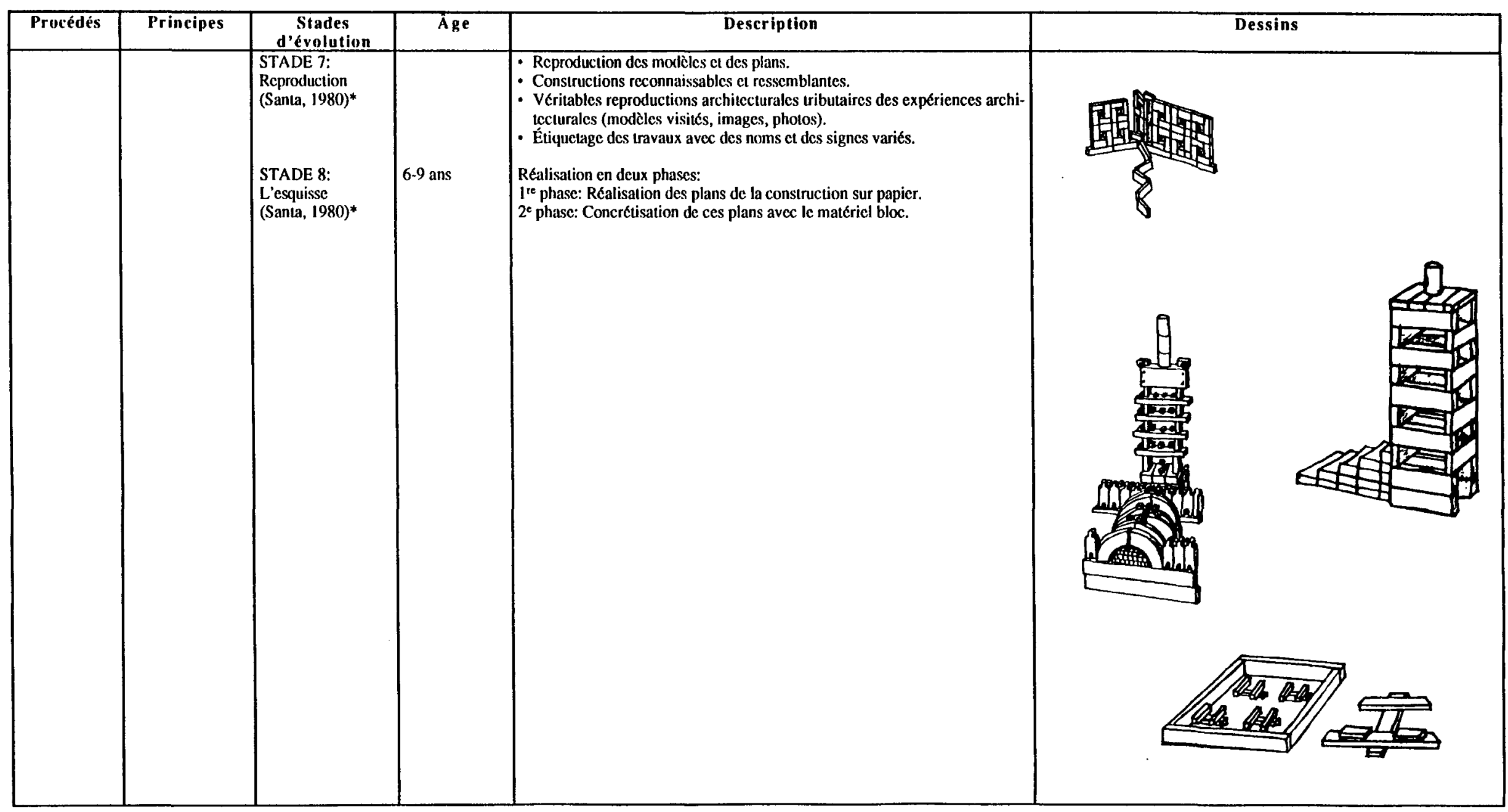

* Les travaux de Mary Ann Santa (1980) s' inscrivent dans la même lipnée des travaux de Johnson mais elle identifie deux stades de plus: la reproduction (7) et l'esquisse (8). 
A.3 Ensembles de projets globaux et secondaires réalisés dans les neuf séances d'observation

\begin{tabular}{|c|c|c|c|c|c|c|}
\hline & & & PROJETS SECONDAIRES & ET CO & PEMENTAIRES & NTRAÎNENT \\
\hline & $\begin{array}{l}\text { CONTEXTE } \\
\text { GLOBAL }\end{array}$ & & $\begin{array}{l}\text { ANSFORMATIONS } \\
\text { MATERIELLES } \\
\end{array}$ & & $\begin{array}{r}\text { TRANSFOR } \\
\text { SYMBOL } \\
\end{array}$ & $\begin{array}{l}\text { TIONS } \\
\text { UES } \\
\end{array}$ \\
\hline Temps & Contexte & Temps & $\begin{array}{c}\text { Représentations } \\
\text { matérielles }\end{array}$ & Temps & $\begin{array}{c}\text { Types de blocs } \\
\text { utilisés }\end{array}$ & $\begin{array}{c}\text { Représentations } \\
\text { symboliques }\end{array}$ \\
\hline $\begin{array}{l}01: 42: 00 \\
03: 41: 00\end{array}$ & $\begin{array}{c}\text { Maison (séance 1, enfant 1) } \\
\downarrow \\
\text { Une belle maison avec } \\
\text { beaucoup d'étages } \\
\end{array}$ & 04:28:00 & Une route & Aucun & & \\
\hline $00: 40: 16$ & $\begin{array}{r}\text { Village de maisons } \\
\text { (séance } 2 \text {, enfant 2) } \\
\text { Modification } \\
\qquad\end{array}$ & $\begin{array}{l}02: 06: 04 \\
31: 09: 16\end{array}$ & $\begin{array}{l}\text { Des chemins à partir de la } \\
\text { maison et autour } \\
\text { Une porte }\end{array}$ & $27: 18: 00$ & & Tracteur \\
\hline $32: 09: 16$ & Un château de sirène & $\begin{array}{l}32: 24: 00 \\
37: 10: 00 \\
37: 46: 00 \\
38: 58: 00 \\
\end{array}$ & $\begin{array}{l}\text { Une porte } \\
\text { Château de la sirène } \\
\text { Auto de la sirène } \\
\text { Une rue } \\
\end{array}$ & $\begin{array}{l}33: 14: 11 \\
39: 41: 12\end{array}$ & & $\begin{array}{l}\text { Petite balançoire } \\
\text { Bassin-piscine }\end{array}$ \\
\hline $00: 02: 13$ & $\begin{array}{c}\text { Maison } \\
\text { (séance 2, enfant 3) }\end{array}$ & & & $\begin{array}{l}08: 48: 01 \\
33: 06: 10 \\
\end{array}$ & & $\begin{array}{l}\text { Porte } \\
\text { Lit }\end{array}$ \\
\hline $00: 15: 00$ & $\begin{array}{c}\text { Maison } \\
\text { (séance 2, enfant 4) }\end{array}$ & $01: 13: 21$ & Le plancher & $\begin{array}{l}07: 21: 13 \\
08: 44: 07 \\
33: 05: 26 \\
34: 11: 18 \\
\end{array}$ & & $\begin{array}{l}\text { Barrière } \\
\text { Balcon, porte } \\
\text { Lit } \\
\text { Cuisine } \\
\end{array}$ \\
\hline 04:48:00 & Garage & \begin{tabular}{|l|}
$04: 57: 00$ \\
$05: 04: 00$ \\
$05: 21: 00$ \\
\end{tabular} & \begin{tabular}{|l} 
Toit \\
Porte \\
Porte de garage \\
\end{tabular} & & & \\
\hline $\begin{array}{l}03: 39: 23 \\
22: 47: 00 \\
\end{array}$ & $\begin{array}{c}\text { Château } \\
\text { (séance } 3 \text {, enfant 1) } \\
\downarrow \\
\text { Château des fantômes } \\
\end{array}$ & & & & & \\
\hline $01: 22: 09$ & $\begin{array}{c}\text { Château } \\
\text { (séance } 3 \text {, enfants } 3 \text { et } 4 \text { ) }\end{array}$ & $\begin{array}{l}00: 59: 11 \\
06: 47: 13 \\
09: 39: 20 \\
10: 49: 10 \\
\\
14: 35: 00 \\
\end{array}$ & $\begin{array}{l}\text { Un salon } \\
\text { Une cuisine } \\
\text { Un fauteuil } \\
\text { Une petite salle } \Rightarrow \text { modification } \\
2^{\circ} \text { Une piscine de crocodile } \Rightarrow> \\
3^{\circ} \text { Un salon } \\
\text { Une piscine dans la maison }\end{array}$ & $01: 42: 29$ & & Brique \\
\hline
\end{tabular}




\begin{tabular}{|c|c|c|c|c|c|c|}
\hline 19:50:00 & Deuxième construction & $\begin{array}{l}10: 15: 15 \\
12: 34: 29 \\
20: 05: 10 \\
20: 43: 07 \\
20: 55: 08 \\
21: 05: 02 \\
21: 23: 23 \\
26: 18: 21\end{array}$ & $\begin{array}{l}\text { Des marches } \\
\text { Une piscine } \\
\text { Le plancher } \\
\text { La cabine } \\
\text { Une cabine à toilette } \\
\text { Une cabine à bol } \\
\text { Salon, cuisine } \\
\text { Une cheminée } \\
\text { La lumière }\end{array}$ & & & \\
\hline $31: 53: 18$ & $\begin{array}{l}\text { Troisième construction } \\
\text { Auto }\end{array}$ & $\begin{array}{l}32: 55: 10 \\
33: 28: 14 \\
33: 36: 14 \\
33: 56: 04 \\
37: 04: 04 \\
40: 02: 02\end{array}$ & $\begin{array}{l}\text { Un envoyeur de fumée } \\
\text { Les vitres } \\
\text { La vitre de l'auto } \\
\text { Les portes } \\
\text { Avant et arrière de l'auto } \\
\text { Les cassettes }\end{array}$ & $\begin{array}{l}32: 55: 10 \\
34: 36: 38 \\
35: 38: 00 \\
36: 12: 12 \\
39: 07: 22 \\
40: 02: 02 \\
40: 10: 55\end{array}$ & $\begin{array}{l}\text { Double module } \\
\text { Courbe elliptique } \\
2 \text { doubles modules } \\
\\
\text { Modules } \\
\text { Modules }\end{array}$ & $\begin{array}{l}\text { Envoyeur de fumée } \\
\text { Volant } \\
\text { Volant } \\
\text { Volant } \\
\text { Un pare-balles } \\
\text { Cassettes } \\
\text { Cassettes }\end{array}$ \\
\hline $\begin{array}{l}00: 15: 00 \\
04: 35: 24\end{array}$ & $\begin{array}{c}\text { Maison } \\
\text { (séance } 5 \text {, enfants } 3 \text { et } 4 \text { ) }\end{array}$ & \begin{tabular}{|l|}
$04: 54: 14$ \\
$06: 32: 07$ \\
$08: 51: 04$ \\
$09: 07: 12$ \\
$09: 23: 09$ \\
$09: 32: 08$ \\
$13: 33: 18$ \\
$18: 10: 10$ \\
$18: 06: 15$ \\
$18: 24: 29$ \\
$20: 26: 08$ \\
$20: 28: 21$ \\
\end{tabular} & \begin{tabular}{|l} 
Barrages \\
Labyrinthe \\
Labyrinthe \\
Labyrinthe en chemins \\
Carré de sable \\
Nature \\
Salle de bain \\
Salle de bain \\
Bancs \\
Bancs \\
Bancs \\
Bancs de secours \\
\end{tabular} & \begin{tabular}{|l|}
$07: 18: 14$ \\
$07: 30: 00$ \\
$07: 51: 10$ \\
$11: 14: 04$ \\
$13: 33: 18$ \\
$19: 42: 18$ \\
$20: 21: 27$ \\
$21: 03: 28$ \\
$21: 38: 10$ \\
$26: 25: 25$ \\
$32: 46: 01$ \\
\end{tabular} & \begin{tabular}{|l|}
$\mathrm{Y}$ \\
$\mathrm{Y}$ \\
Bassin \\
Pointe un espace \\
Cylindre \\
Pointe un espace \\
\\
Demi-arche romaine \\
Triangle \\
Triangle \\
\end{tabular} & $\begin{array}{l}\text { Petite porte } \\
\text { Porte-patio } \\
\text { Porte-patio } \\
\text { Petit lac } \\
\text { Salle de bain } \\
\text { Batterie } \\
\text { Cheminée } \\
\text { Berceau du bébé } \\
\text { Système d'alarme } \\
\text { Marteau } \\
\text { Clou } \\
\text { Clou informatique } \\
\end{array}$ \\
\hline $00: 57: 06$ & (séance 5 , enfant 4 ) & $\begin{array}{l}00: 33: 24 \\
02: 48: 13 \\
02: 58: 05 \\
05: 46: 20 \\
07: 59: 01 \\
06: 08: 24 \\
11: 21: 05 \\
13: 43: 25 \\
15: 11: 20 \\
17: 17: 09 \\
18: 03: 02\end{array}$ & \begin{tabular}{|l|} 
Cheminée \\
Bancs \\
Clôtures \\
Labyrinthe \\
Labyrinthe \\
Porte secrète \\
Lac \\
Toilette \\
Salle de bain \\
Barrage \\
Bancs \\
\end{tabular} & \begin{tabular}{|l|}
$39: 10: 29$ \\
$39: 27: 17$ \\
$39: 32: 25$ \\
$39: 40: 27$ \\
$40: 42: 14$
\end{tabular} & $\begin{array}{l}5 \text { blocs carrefour } \\
\text { Carrefour } \\
\text { Chaussée }\end{array}$ & \begin{tabular}{|l|} 
Eau \\
Eau \\
Crocodiles \\
Crocodiles \\
Labyrinthe de crocro
\end{tabular} \\
\hline
\end{tabular}




\begin{tabular}{|c|c|c|c|c|c|c|}
\hline $00: 57: 06$ & $\begin{array}{c}\text { (séance 5, enfant 4) } \\
\text { (suite) }\end{array}$ & $\begin{array}{l}18: 27: 29 \\
19: 50: 10 \\
21: 14: 17 \\
22: 42: 10\end{array}$ & $\begin{array}{l}\text { Bancs de présidence } \\
\text { Bancs de sécurité } \\
\text { Piano } \\
\text { Piano de sécurité } \\
\text { Salle de gymnastique }\end{array}$ & & & \\
\hline $\begin{array}{l}00: 15: 00 \\
04: 35: 24\end{array}$ & $\begin{array}{c}\text { Château } \\
\text { (séance } 6 \text {, enfant 4) } \\
\downarrow \\
\text { Royaume }\end{array}$ & & & $04: 09: 02$ & Double module & Mitraillette \\
\hline & $\begin{array}{c}\text { Château } \\
\text { (séance } 7 \text {, enfant } 1 \text { ) }\end{array}$ & & & $24: 27: 22$ & & Radio \\
\hline $00: 57: 06$ & $\begin{array}{c}\text { Château } \\
\text { (séance } 7, \text { enfant } 2 \text { ) }\end{array}$ & $\begin{array}{l}01: 05: 18 \\
03: 22: 18 \\
03: 58: 05 \\
07: 13: 00 \\
\end{array}$ & $\begin{array}{l}\text { Plancher } \\
\text { Tour } \\
\text { Passerelles } \\
\text { Des étages } \\
\end{array}$ & & & \\
\hline $\begin{array}{l}00: 04: 02 \\
23: 43: 04 \\
\end{array}$ & $\begin{array}{c}\text { Poste de police } \\
\text { (séance } 7 \text {, enfant 3) } \\
\downarrow \\
\text { Fourrière pour les animaux }\end{array}$ & $\begin{array}{l}02: 09: 00 \\
02: 12: 22 \\
03: 39: 12 \\
05: 05: 03 \\
10: 44: 05\end{array}$ & $\begin{array}{l}\text { Murs } \\
\text { Entrée } \\
\text { Parking } \\
\text { Enclos } \\
\text { Clôture }\end{array}$ & \begin{tabular}{|l|}
$01: 55: 02$ \\
$02: 04: 05$ \\
$13: 00: 05$ \\
$16: 28: 16$ \\
$11: 16: 22$ \\
$19: 14: 17$ \\
\end{tabular} & $\begin{array}{l}\text { Carrefour } \\
\text { Colonne } \\
\text { Panneau de signalisation } \\
\text { Autos } \\
\text { Hélicoptère de police }\end{array}$ & $\begin{array}{l}\text { Bonhomme de police } \\
\text { Fenêtre } \\
\text { Radar } \\
\text { Girouette } \\
\text { Toutes des autos de police }\end{array}$ \\
\hline $00: 33: 25$ & $\begin{array}{c}\text { Poste de police } \\
\text { (séance } 7, \text { enfant } 4 \text { ) }\end{array}$ & $\begin{array}{l}02: 06: 05 \\
02: 24: 06 \\
03: 51: 11 \\
14: 13: 13\end{array}$ & $\begin{array}{l}\text { Entrée, en arrière } \\
\text { Garage } \\
\text { Garage de pompiers } \\
\text { Clôture }\end{array}$ & $\begin{array}{l}03: 10: 20 \\
10: 40: 13 \\
12: 58: 17 \\
14: 01: 00 \\
14: 58: 12 \\
16: 16: 25 \\
17: 20: 06 \\
18: 03: 10 \\
\end{array}$ & $\begin{array}{l}\text { Auto } \\
\text { Module } \\
\text { Double colonne } \\
\text { Double colonne } \\
\\
\text { Double module } \\
\text { Double module } \\
\end{array}$ & $\begin{array}{l}\text { Auto de police } \\
\text { Radar } \\
\text { Lumière du radar } \\
\text { Cheminée } \\
\text { Barrière } \\
\text { Une affaire pour se "tinker" } \\
1^{\circ} \text { cheminée, } 2^{\circ} \text { l'équerre } \\
\text { Lumière } \\
\end{array}$ \\
\hline $00: 39: 17$ & $\begin{array}{c}\text { Château } \\
\text { (séance } 9, \text { enfant 2) }\end{array}$ & $\begin{array}{l}03: 34: 11 \\
07: 56: 01\end{array}$ & $\begin{array}{l}\text { Garage } \\
\text { Le piège }\end{array}$ & $25: 06: 05$ & Carrefour & Crocodiles \\
\hline $02: 41: 10$ & $\begin{array}{c}\text { Château } \\
\text { (séance 9, enfant 4) }\end{array}$ & $\begin{array}{l}04: 47: 00 \\
09: 57: 08 \\
16: 47: 16\end{array}$ & $\begin{array}{l}\text { Chaise } \\
\text { Bouton (manettes) } \\
\text { Canon }\end{array}$ & $\begin{array}{l}09: 57: 08 \\
29: 20: 14 \\
29: 56: 03 \\
\end{array}$ & $\begin{array}{l}\text { Panneaux de signalisa- } \\
\text { tion }\end{array}$ & $\begin{array}{l}\text { Manettes } \\
\text { Bombe à rechargeurs } \\
\text { Bombes }\end{array}$ \\
\hline
\end{tabular}




\begin{tabular}{|c|c|c|c|c|c|c|}
\hline $\begin{array}{l}00: 58: 19 \\
19: 14: 21 \\
31: 32: 19\end{array}$ & $\begin{array}{c}\text { Maison } \\
\text { (séance 8, enfants } 1 \text { et } 2 \text { ) } \\
\downarrow \\
\text { Zoo } \\
\downarrow \\
\text { Château d'armée }\end{array}$ & $\begin{array}{l}19: 14: 21 \\
21: 26: 03 \\
23: 53: 53 \\
28: 55: 15 \\
26: 27: 06\end{array}$ & $\begin{array}{l}\text { Barrière du zoo } \\
\text { C'est une chose pour les féroces } \\
\text { Une barrière aux animaux } \\
\text { Porte } \\
\text { Caverne de malins }\end{array}$ & $\begin{array}{l}02: 38: 02 \\
07: 38: 05 \\
10: 42: 10 \\
\\
18: 10: 27 \\
19: 14: 21 \\
35: 05: 06\end{array}$ & $\begin{array}{l}\text { Grande planche de cons- } \\
\text { truction } \\
2 \text { bonhommes de bois } \\
\text { colorés } \\
\text { Quadrangle } \\
\text { L'espace libre de la } \\
\text { construction }\end{array}$ & $\begin{array}{l}\text { Une chose d'auto (station- } \\
\text { nement) } \\
\text { Barrière } \\
2 \text { gardes (gardiens) } \\
\text { Barrière des animaux } \\
\text { Barrière du zoo } \\
\text { Prison }\end{array}$ \\
\hline $\begin{array}{l}09: 06: 05 \\
09: 16: 16 \\
13: 54: 03\end{array}$ & $\begin{array}{c}\text { (séance 8, enfants } 3 \text { et } 4 \text { ) } \\
\text { Maison } \\
\downarrow \\
\text { Château } \\
\downarrow \\
\text { Château-maison }\end{array}$ & $\begin{array}{l}09: 16: 16 \\
15: 21: 09 \\
15: 46: 28 \\
16: 24: 01 \\
17: 48: 06 \\
18: 29: 23 \\
20: 09: 18 \\
25: 00: 29 \\
25: 09: 06 \\
26: 23: 14 \\
28: 32: 10 \\
30: 33: 12 \\
31: 21: 26 \\
33: 44: 20 \\
37: 47: 15 \\
37: 23: 22 \\
28: 39: 07 \\
31: 35: 03 \\
34: 00: 25 \\
34: 20: 00 \\
35: 01: 25 \\
37: 44: 02 \\
43: 50: 17 \\
\end{array}$ & \begin{tabular}{l|} 
Plancher \\
La porte \\
Porte \\
Porte-patio en arrière, porte du \\
côté \\
Porte \\
La toilette \\
Arc-en-ciel \\
Antenne de T.V. \\
Antenne de T.V. \\
Des sièges \\
Bancs \\
Bancs \\
Gros bancs \\
Le divan \\
Le divan \\
Une fenêtre \\
Porte-patio \\
Fenêtre à rideau \\
Antenne de télé \\
Une fenêtre \\
Fenêtre \\
Divan \\
Antenne \\
\end{tabular} & $\begin{array}{l}10: 35: 26 \\
11: 03: 16 \\
14: 07: 03 \\
14: 40: 03 \\
17: 48: 06 \\
28: 59: 13 \\
\\
10: 39: 04 \\
14: 50: 21\end{array}$ & $\begin{array}{l}\text { Demi-carrefour } \\
2 \text { triangles } \\
2 \text { carrefours } \\
\text { Triangle } \\
2 \text { gros cylindres } \\
2 \text { grands cylindres }\end{array}$ & $\begin{array}{l}\text { Eau, eau froide, eau chaude } \\
\text { Robinet } \\
\text { Oiseau } \\
\text { Eau chaude, eau froide } \\
\text { T.V. } \\
\text { Pinceau pour peindre la } \\
\text { maison rose fluo } \\
\text { Pour vider l'eau } \\
\text { Contenant pour boire }\end{array}$ \\
\hline
\end{tabular}




\begin{tabular}{|c|c|c|c|c|c|c|}
\hline \multirow{2}{*}{\multicolumn{2}{|c|}{$\begin{array}{l}\text { CONTEXTE } \\
\text { GLOBAL }\end{array}$}} & \multicolumn{5}{|c|}{ PROJETS SECONDAIRES ET COMPLEMENTAIRES ENTRAÎNENT } \\
\hline & & \multicolumn{2}{|r|}{$\begin{array}{l}\text { TRANSFORMATIONS } \\
\text { MATÉRIELLES }\end{array}$} & \multicolumn{3}{|c|}{$\begin{array}{l}\text { TRANSFORMATIONS } \\
\text { SYMBOLIQUES } \\
\end{array}$} \\
\hline Temps & Contexte & Temps & $\begin{array}{l}\text { Représentations } \\
\text { matérielles }\end{array}$ & Temps & $\begin{array}{l}\text { Types de blocs } \\
\text { utilisés }\end{array}$ & $\begin{array}{l}\text { Représentations } \\
\text { symboliques }\end{array}$ \\
\hline $01: 57: 14$ & $\begin{array}{c}\text { Bateau à moteur } \\
\text { (séance } 4 \text {, enfant } 4 \text { ) }\end{array}$ & $\begin{array}{l}02: 21: 14 \\
03: 30: 17 \\
07: 49: 05 \\
14: 22: 25 \\
15: 48: 11\end{array}$ & \begin{tabular}{|l} 
Un moteur \\
Une cabine \\
Une cheminée \\
Pont intérieur \\
Escalier
\end{tabular} & $\begin{array}{l}04: 29: 27 \\
07: 56: 22 \\
07: 56: 22 \\
12: 02: 21 \\
13: 03: 28 \\
23: 54: 17 \\
25: 22: 00 \\
27: 43: 05 \\
30: 23: 20 \\
31: 19: 27 \\
36: 50: 12 \\
\end{array}$ & $\begin{array}{l}\text { Planche de toit } \\
\text { Quadrangle } \\
\text { Grand cylindre } \\
\text { Colonne double } \\
\text { Colonne double } \\
\\
\text { Cylindre } \\
3 \text { cylindres } \\
\text { Colonne } \\
2 \text { quadrangles }\end{array}$ & $\begin{array}{l}\text { Moteur } \\
2 \text { cheminées } \\
\text { Cabine } \\
\text { Dynamites } \\
\text { Bloc de dynamite } \\
\text { Verseur d'huile } \\
\text { Bombe } \\
\text { Manette } \\
\text { Gaz } \\
3 \text { bouteilles d'eau } \\
\text { Paquet d'allumettes } \\
\text { Sièges }\end{array}$ \\
\hline $\begin{array}{c}00: 07: 00 \\
01: 47: 29\end{array}$ & $\begin{array}{c}\text { Auto } \\
\downarrow \\
\text { Bateau }\end{array}$ & $\begin{array}{l}02: 01: 16 \\
08: 44: 26 \\
20: 07: 00 \\
\\
20: 39: 20 \\
28: 39: 10 \\
40: 04: 14 \\
41: 36: 22\end{array}$ & $\begin{array}{l}\text { Plancher } \\
\text { Une cabine } \\
\text { Toutes sortes de dynamites } \\
\text { Fusils } \\
\text { Fusil } \\
\text { Table } \\
\text { Robot } \\
\text { Échasses }\end{array}$ & \begin{tabular}{|l|}
$04: 08: 00$ \\
$11: 48: 17$ \\
$11: 55: 21$ \\
$14: 07: 22$ \\
$29: 46: 15$ \\
$37: 17: 23$ \\
$39: 09: 27$ \\
$39: 27: 05$ \\
$42: 32: 21$ \\
\end{tabular} & $\begin{array}{l}\text { Courbe elliptique } \\
\text { Double colonne } \\
2 \text { colonnes } \\
1 \text { colonne } \\
\text { Grand cylindre } \\
\text { Modules } \\
\text { Saute d'un bloc à l'autre } \\
\text { Colonne } \\
\end{array}$ & $\begin{array}{l}\text { Volant } \\
\text { Volant } \\
\text { Dynamites } \\
\text { Bâton de dynamite } \\
\text { Bombe } \\
\text { Dynamite } \\
\text { Pont } \\
\text { Boucane } \\
\text { Cigarettes } \\
\end{array}$ \\
\hline $\begin{array}{l}00: 03: 28 \\
19: 40: 04\end{array}$ & $\begin{array}{c}\text { Bâtisse } \\
\text { (séance } 4 \text {, enfant 3) } \\
\downarrow \\
\text { Bateau } \\
\downarrow \\
\text { Bateau de dynamites }\end{array}$ & $14: 12: 24$ & Une tour & $\begin{array}{l}06: 06: 08 \\
07: 25: 05 \\
18: 37: 16 \\
19: 44: 09 \\
22: 09: 04 \\
27: 53: 02 \\
29: 52: 00 \\
30: 58: 23 \\
31: 38: 00 \\
34: 37: 22 \\
\\
41: 55: 02 \\
41: 18: 19\end{array}$ & $\begin{array}{l}\text { Modules } \\
2 \text { courbes elliptiques } \\
\text { Quadrangle } \\
\text { Module } \\
\text { Demi-module } \\
\text { Demi-module } \\
\text { Colonne } \\
2 \text { colonnes } \\
\\
\text { Demi-module } \\
\text { Planche de toit }\end{array}$ & $\begin{array}{l}\text { "Mufflers" } \\
\text { Envoyeur de fumée } \\
\text { Moteur à bateau } \\
\text { Dynamites } \\
\text { Mes boucanes } \\
\text { Huile } \\
\text { Bâton de dynamite } \\
\text { Verre } \\
\text { Bâtons de dynamite } \\
\text { Frotte ensemble pour allu- } \\
\text { mer } \\
\text { Paquet d'allumettes } \\
\text { Dynamites }\end{array}$ \\
\hline
\end{tabular}




\begin{tabular}{|c|c|c|c|c|c|c|}
\hline $00: 53: 12$ & $\begin{array}{c}\text { Château-bateau } \\
\text { (séance } 5 \text {, enfant } 1 \text { ) }\end{array}$ & $\begin{array}{l}05: 12: 04 \\
22: 37: 29 \\
27: 23: 18 \\
35: 20: 14 \\
35: 23: 01 \\
38: 18: 12 \\
29: 19: 02 \\
37: 33: 29 \\
37: 29: 16 \\
32: 21: 26 \\
33: 00: 15\end{array}$ & $\begin{array}{l}\text { Des labyrinthes } \\
\text { Salle de bain } \\
\text { Bancs } \\
\text { Bancs } \\
\text { Bancs } \\
\text { Bancs } \\
\text { Table } \\
\text { Table } \\
\text { Table } \\
\text { Porte } \\
\text { Table et bancs }\end{array}$ & $\begin{array}{l}03: 58: 18 \\
04: 53: 10 \\
11: 14: 20 \\
11: 36: 00 \\
13: 43: 06 \\
15: 48: 11 \\
17: 01: 00 \\
18: 07: 23 \\
26: 56: 24 \\
32: 56: 07 \\
38: 53: 08 \\
39: 34: 17 \\
\end{array}$ & $\begin{array}{l}\text { Planche de toit } \\
\text { Quadrangle } \\
3 \text { grands cylindres } \\
2 \text { courbes elliptiques } \\
2 \text { courbes elliptiques } \\
2 \text { courbes elliptiques } \\
\text { Colonne double } \\
\text { Carrefours et chaussée } \\
\text { Chaussée } \\
\end{array}$ & $\begin{array}{l}\text { Porte pour entrer } \\
\text { Entrée } \\
\text { Envoyeur de fumée } \\
\text { Petit oiseau } \\
\text { Cheminée } \\
\text { Des petits ponts } \\
\text { Pour la douche } \\
\text { La porte de salle de bain } \\
\text { Piano } \\
\text { Lit } \\
\text { Eau } \\
\text { Crocodiles }\end{array}$ \\
\hline & (séance 5 , enfant 2 ) & $\begin{array}{l}04: 53: 10 \\
05: 40: 24 \\
08: 49: 21 \\
09: 18: 29 \\
09: 25: 04 \\
09: 33: 21 \\
10: 35: 10 \\
12: 46: 08 \\
11: 09: 26 \\
13: 19: 23 \\
13: 45: 21 \\
13: 52: 28 \\
\\
15: 48: 23 \\
19: 03: 11\end{array}$ & $\begin{array}{l}\text { Entrée } \\
\text { Labyrinthes } \\
\text { Labyrinthe } \\
\text { Un carré de sable } \\
\text { On dirait une voiture } \\
\text { Voiture pour embarquer à quatre } \\
\text { Bancs } \\
4 \text { bancs } \\
\text { Eau } \\
\text { Salle de bain } \\
\text { La toilette } \\
\text { La cheminée } \\
\text { L'oiseau } \\
\text { Une petite porte } \\
\text { La toilette } \\
\text { le robinet }\end{array}$ & $\begin{array}{l}06: 27: 12 \\
07: 22: 02 \\
14: 33: 22 \\
23: 41: 10\end{array}$ & $\begin{array}{l}\text { Quadrangle } \\
\text { Quadrangle } \\
\text { Cylindres } \\
\text { Cylindres }\end{array}$ & $\begin{array}{l}\text { Porte } \\
\text { Porte-patio } \\
\text { Bâtons } \\
\text { Système d'alarme pour la } \\
\text { cheminée }\end{array}$ \\
\hline
\end{tabular}




\begin{tabular}{|c|c|c|c|c|c|c|}
\hline 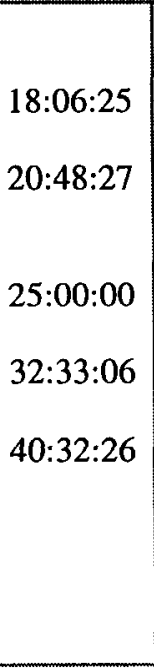 & $\begin{array}{c}\text { Château } \\
\downarrow \\
\text { C'est un bateau privé } \\
\downarrow \\
\text { Un bateau d'espions } \\
\text { Un bateau de voleurs } \\
\downarrow \\
\text { Bateau tigre } \\
\downarrow \\
\text { Bateau de secours } \\
\downarrow \\
\text { bateau de secours }\end{array}$ & $04: 47: 00$ & Une chaise & \begin{tabular}{l|}
$32: 02: 29$ \\
$34: 13: 09$ \\
$34: 37: 26$ \\
$35: 35: 17$ \\
$35: 42: 27$ \\
$35: 48: 01$ \\
$36: 05: 19$ \\
$36: 21: 10$ \\
$38: 27: 16$ \\
$39: 39: 07$ \\
$39: 53: 10$ \\
$40: 15: 07$ \\
$40: 56: 03$ \\
$41: 26: 08$ \\
$41: 47: 25$ \\
$42: 30: 20$ \\
\end{tabular} & $\begin{array}{l}\text { Triangle } \\
\text { Quadrangles } \\
\text { Quadrangles } \\
\text { Quadrangles } \\
\text { Double module } \\
\\
4 \text { quadrangles }\end{array}$ & $\begin{array}{l}\text { Bombes à rechargeurs } \\
\text { Bombes rechargeuses } \\
\text { Bombes } \\
\text { Bombe explosante } \\
\text { Fusils } \\
\text { Bazookas } \\
\text { Bazookas } \\
\text { Bombes } \\
\text { Bombes } \\
\text { Bombes } \\
\text { Fusils } \\
\text { Bateaux } \\
\text { Coup de fusil } \\
\text { Des bateaux } \\
4 \text { bateaux } \\
\text { Bateaux qui explosent } \\
\text { Ski nautique }->\text { bombes }\end{array}$ \\
\hline $\begin{array}{c}17: 31: 19 \\
42: 03: 22\end{array}$ & $\begin{array}{c}\text { Bateau } \\
\text { (séance } 9, \text { enfant 3) } \\
\downarrow \\
\text { Bateau de canon } \\
\downarrow \\
\text { Vaisseau }\end{array}$ & $15: 13: 14$ & Canon & $\begin{array}{l}16: 53: 00 \\
17: 31: 19 \\
28: 34: 18 \\
31: 14: 02 \\
35: 29: 03 \\
35: 43: 07 \\
36: 10: 21 \\
37: 27: 29 \\
39: 50: 03 \\
40: 58: 13 \\
\end{array}$ & $\begin{array}{l}\text { Gros cylindre } \\
\text { Gros cylindres } \\
\text { Colonne double } \\
\text { Double module }\end{array}$ & $\begin{array}{l}\text { Poudre de canon - bombe } \\
\text { Bateau de canon } \\
\text { Boulet de canon } \\
\text { Bombes pas rechargeuses } \\
\text { Bombe comme des pétards } \\
\text { Bazookas } \\
\text { Bombes chargées } \\
\text { Bombe } \\
\text { Vaisseau } \\
\text { Bombes }\end{array}$ \\
\hline $\begin{array}{l}00: 10: 14 \\
04: 47: 02\end{array}$ & $\begin{array}{c}\text { Château } \\
\text { (séance } 9 \text {, enfant 1) } \\
\downarrow \\
\text { Bateau }\end{array}$ & $\begin{array}{l}00: 46: 28 \\
04: 57: 04\end{array}$ & $\begin{array}{l}\text { Porte } \\
\text { Chaise }\end{array}$ & $\begin{array}{l}02: 07: 03 \\
03: 04: 07 \\
06: 27: 03 \\
09: 15: 27 \\
\\
21: 28: 20 \\
34: 17: 27 \\
35: 09: 15 \\
35: 15: 01 \\
36: 25: 20 \\
37: 44: 29 \\
38: 46: 15 \\
40: 17: 19 \\
\end{array}$ & $\begin{array}{l}\text { Demi-module } \\
\text { Demi-module } \\
\text { Colonne } \\
2 \text { panneaux de } \\
\text { signalisation } \\
\\
\text { Quadrangles } \\
\text { Quadrangles } \\
\text { Triangles } \\
\\
\text { Porte gothique }\end{array}$ & $\begin{array}{l}\text { Bombes } \\
\text { Bombes } \\
\text { Bombes } \\
\text { Manettes } \\
\\
\text { Boulet de canon } \\
\text { Bombes } \\
\text { Bombes, dynamites } \\
\text { Grosses bombes } \\
\text { Bombes } \\
\text { Bombes } \\
\text { Fusils } \\
\text { Bazookas } \\
\text { La plus grosse bombe }\end{array}$ \\
\hline
\end{tabular}




\begin{tabular}{|c|c|c|c|c|c|c|}
\hline \multirow{2}{*}{\multicolumn{2}{|c|}{$\begin{array}{l}\text { CONTEXTE } \\
\text { GLOBAL }\end{array}$}} & \multicolumn{5}{|c|}{ PROJETS SECONDAIRES ET COMPLEMENTAIRES ENTRAÎNENT } \\
\hline & & \multicolumn{2}{|c|}{$\begin{array}{l}\text { TRANSFORMATIONS } \\
\text { MATÉRIELLES } \\
\end{array}$} & \multicolumn{3}{|c|}{$\begin{array}{c}\text { TRANSFORMATIONS } \\
\text { SYMBOLIQUES } \\
\end{array}$} \\
\hline Temps & Contexte & Temps & $\begin{array}{l}\text { Représentations } \\
\text { matérielles }\end{array}$ & Temps & $\begin{array}{c}\text { Types de blocs } \\
\text { utilisés }\end{array}$ & $\begin{array}{l}\text { Représentations } \\
\text { symboliques }\end{array}$ \\
\hline & $\begin{array}{l}\text { Aucune annonce } \\
\text { (séance } 4 \text {, enfant } 1 \text { ) }\end{array}$ & $12: 41: 00$ & Banc & \begin{tabular}{|l|}
$07: 22: 00$ \\
$33: 19: 00$ \\
$35: 45: 17$ \\
\end{tabular} & $\begin{array}{l}\text { Blocs } \\
2 \text { courbes elliptiques } \\
\text { Demi-cercle } \\
\end{array}$ & $\begin{array}{l}\text { Plancher escalier } \\
2 \text { portes de voiture } \\
\text { Volant de voiture } \\
\end{array}$ \\
\hline $\begin{array}{l}15: 17: 08 \\
19: 02: 20\end{array}$ & $\begin{array}{c}\text { Grosse base } \\
\text { (séance } 6 \text {, enfant 2) } \\
\downarrow \\
\text { Édifice } \\
\downarrow \\
7 \text { étages } \\
\end{array}$ & $30: 18: 00$ & $\begin{array}{l}\text { Autre bâtisse } \\
\text { l'escalier }\end{array}$ & 28:06:01 & $\begin{array}{l}\text { Double module } \\
\text { Quadrangle }\end{array}$ & $\begin{array}{l}\text { Entrée } \\
\text { Porte secrète pour entrer les } \\
\text { voitures }\end{array}$ \\
\hline $\begin{array}{l}00: 18: 24 \\
\\
17: 53: 18 \\
23: 51: 14 \\
34: 33: 09 \\
34: 56: 27\end{array}$ & $\begin{array}{c}\text { Autoroute } \\
\text { (séance 6, enfant 3) } \\
\downarrow \\
\text { Ferme } \\
\text { Pas de ferme } \\
\downarrow \\
\text { Maison } \\
\downarrow \\
\text { Garage }\end{array}$ & $\begin{array}{l}03: 43: 05 \\
10: 22: 29 \\
30: 19: 17 \\
34: 00: 21\end{array}$ & \begin{tabular}{|l|} 
Pont \\
Gros pont \\
Escalier \\
Porte secrète
\end{tabular} & \begin{tabular}{|l}
$34: 33: 09$ \\
$34: 05: 16$ \\
$27: 30: 00$ \\
\\
$42: 54: 19$
\end{tabular} & $\begin{array}{l}\text { Quadrangle } \\
\text { Quadrangle } \\
\text { Double module }\end{array}$ & $\begin{array}{l}\text { Porte } \\
\text { Passage } \\
\text { Pente pour monter sur } \\
\text { l'édifice } \\
\text { Porte }\end{array}$ \\
\hline
\end{tabular}


A.4 Identification de l'ensemble des rôles nommés initiés et joués dans l'activité de jeu symbolique subordonnée à l'activité de construction selon la classification de Garvey (1977)

\begin{tabular}{|c|c|c|c|c|c|c|c|}
\hline $\begin{array}{c}\text { Rôles } \\
\text { périphériques: } \\
\text { de caractère } \\
\text { de fiction }\end{array}$ & $\begin{array}{c}\text { Rôles } \\
\text { familiaux } \\
\text { implicites: }\end{array}$ & \begin{tabular}{|c|}
\multicolumn{1}{c|}{ Rôles } \\
fonctionnels \\
périphériques \\
de caractère \\
stéréotypé:
\end{tabular} & $\begin{array}{c}\text { Rôles } \\
\text { fonctionnels } \\
\text { périphériques } \\
\text { de } \\
\text { personnages- } \\
\text { animaux: }\end{array}$ & $\begin{array}{c}\text { Rôles de } \\
\text { caractère } \\
\text { stéréotypé } \\
\text { avec trame } \\
\text { évolutive très } \\
\text { peu } \\
\text { complexe: } \\
\end{array}$ & $\begin{array}{c}\text { Rôles de } \\
\text { caractère } \\
\text { stéréotypé: }\end{array}$ & $\begin{array}{l}\text { Rôles de } \\
\text { caractère de } \\
\text { fiction: }\end{array}$ & $\begin{array}{l}\text { *Rôles de } \\
\text { personnage- } \\
\text { animal: }\end{array}$ \\
\hline $\begin{array}{ll}\text { - } & \text { sirène } \\
\text { - roi } \\
\text { - géant }\end{array}$ & - enfants & $\begin{array}{l}\text { - armée (soldats } \\
\text { qui font éclater } \\
\text { des bombes) } \\
\text { - enf } \\
\text { ants/professeur } \\
\text { - chauffeur } \\
\text { d'autobus } \\
\sim \text { attaqueur } \\
\text { - voleur }\end{array}$ & $\begin{array}{ll}\text { - } & \text { gorille } \\
\text { - tigre } \\
\text { - d'autres } \\
\text { animaux }\end{array}$ & 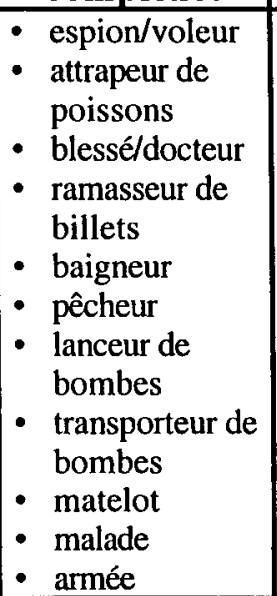 & $\begin{array}{l}\text { - conducteur } \\
\text { - } \text { esclave } \\
\text { - capitaine } \\
\text { - } \text { ennememi/guide } \\
\text { - } \text { voleur } \\
\text { - } \text { brisonnier } \\
\text { - mort }\end{array}$ & - pirate & $\begin{array}{l}\text { - } \text { singe } \\
\text { - le roi des } \\
\text { animaux: lion } \\
\text { - le patron: lion } \\
\text { - le chef: lion } \\
\text { - rôles } \\
\text { d'animaux qui } \\
\text { travaillent } \\
\text { pour eux: } \\
\text { éléphant, } \\
\text { chameau }\end{array}$ \\
\hline
\end{tabular}

* Rôles non identifiés par Garvey. 


\section{BIBLIOGRAPHIE}

Almy, M., P. Monighan, B. Scales et J. Van Hoorn. 1982. Recent Research on Play: The Perspective of the Teacher. Washington, DC: The National Institute of Education.

Anadon, M. 1988. Épistémologie et recherche en éducation. Chicoutimi: UQAC (texte inédit).

Apelman, M. 1984. «The Stage of Block Building». In The Block Book, sous la direction de E.S. Hirsch, p. 38-41. Washington: The National Association for the Education of Young Children.

Bailey, M. 1933. «A Scale of Block Constructions for Young Children». Child Development, vol. 4, p. 121-139.

Ballion, M., M. Bréauté, S. Rayna et M. Stamback. 1987. «Des enfants de moins de quatre ans entre eux: observations réciproques, négociations et échanges de points de vue». In D. Zimmerman (Ed), On n'apprend pas tout seul. Interactions sociales et construction des savoirs. Paris: Les Editions E.S.F.

Ballion, M., L. Bonica, M. Bréauté, S. Mayer, T. Musatti, S. Rayna, H. Sinclair, M. Stamback et M. Verba. 1990. Les jeux de fiction entre enfants de 3 ans. Paris: Presses universitaires de France, 202 p.

Bandura, A. et R.H. Walters. 1963. Social Learning and Personality Development. New York: Holt, Rinehart and Winston.

Bandura, A. 1971. Social Learning Theory. New York: General Learning Press.

Bandura, A. 1977. Social Learning Theory. Englewood Cliffs, N.J.: Prentice-Hall.

Barnier, G. 1987. L'effet tuteur dans des situations mettant en jeu des rapports spatiaux. Mémoire de D.E.A.. Aix-en-Provence: Université de Provence.

Bateson, G. 1955. «Theory of Play and Fantasy». Psychiatric Research Reports, vol. 2, p. 39-51. 
Bateson, G. 1956. «The Message "This Is Play"». In Group Process, sous la dir. de B. Schaffner. New York: Josiah Macy.

Beattie, I.D. 1986. «Building Understanding with Blocks». The Arithmetic Teacher, vol. $34, n^{\circ} 2$, p. $5-11$.

Beaudichon, J. 1985. «Que mesure-t-on dans les expériences consacrées au développement de la communication référentielle?». In G. Noizet, D. Bélanger et F. Bresson (Eds), La communication, p. 137-161. Paris: P.U.F.

Beaudichon, J. et C. Vandesplas-Holper. 1985. «Analyse des interactions et de leur effet dans la communication référentielle et la maîtrise de notions». In G. Mugny (Ed), Psychologie sociale du développement cognitif, p. 125-149. Berne: Peter Lang.

Beeson, B. et R.A. Williams. 1979, 1980. A Study of Sex Stereotyping in ChildSelected Play Activities of Preschool Children. Muncie, In: Ball State University, 58 p. (novembre 1979).

Black, B. 1989. «Interactive Pretense: Social and Symbolic Skills in Preschools Play Groups». Merril-Palmer Quartely (octobre), vol. 35, n² 4, p. 379-397.

Black, B. et N.L. Hazen. 1990. «Social Status and Pattern of Communication in Acquainted and Unacquainted Preschool Children». Developmental Psychology, vol. 26, p. $379-387$.

Black, B. 1992. «Negociating Social Pretend Play: Communication. Differences Related to Social Status and Sex». Merril-Palmer Quartely (April), vol. 38, n², p. 212239.

Blackman, N. 1977. An Investigation of the Relation of Historical Change to the Sexual Identification of the Pre-Adolescent as Seen in Dramatic Block Play. Unpublished doctoral dissertation. Maryland: University of Maryland.

Blaye, A. 1986. «Confrontation socio-cognitive et organisation du produit de deux ensembles». Cahiers de psychologie cognitive, vol. 6, p. 87-94.

Blaye, A. 1988. «Mécanismes générateurs de progrès lors de la résolution à deux du produit de deux ensembles par des enfants de 5-6 ans». In A.N. Perret-Clermont (Ed), Interagir et connaître: enjeux et régulations sociales dans le développement cognitif, p. 55-71. Cousset (Fribourg): Delval.

Bonica, L. 1990. «Négociations interpersonnelles et jeux de fiction». In Les jeux de fiction entre enfants de 3 ans, sous la dir. de M. Stamback et H. Sinclair, p. 113151. Paris: Presses universitaires de France.

Brody, C. 1984. «Social Studies and Self-awareness». In The Block Book, sous la dir. de E. Hirsch, p. 103-119. U.S.: National Association for the Education of Young Children. 
Brougère, G. 1984a. «Le jouet aujourd'hui: un objet industriel parmi d'autres?» La marge, $\mathrm{n}^{\circ} 6$ (Mai), p. 79-90. Association nationale des directeurs d'établissements et Services pour inadaptés.

Brougère, G. 1984b. Le jouet comme média culturel. Belgique: Troisième congrès international des ludothèques: «Jouer... communiquen».

Brougère, G. 1987. «Le rôle du jouet dans l'imprégnation culturelle de l'enfant». $16^{e}$ Colloque de l'I.C.C.P., Suhl (octobre).

Brougère, G. 1989. Que peut le jeu? $3^{\mathrm{e}}$ congrès FNAREM, Besançon.

Bruner, J.S., A. Jolly et K. Sylva (Eds). 1976. Play - its Role in Development and Evolution. New York: Basic Books.

Bruni, J.V. et V.H.J. Silverman. 1977. «From Blocks and Model Making to Ratio and Proportion». The Arithmetic Teacher, vol. 24, $\mathrm{n}^{\circ}$ 3, p. 172-180.

Campagne, F. 1989. Le jouet, l'enfant, l'éducateur. Rôle de l'objet dans le développement de l'enfant et le travail pédagogique. Toulouse: Éditions Privat, 118 p.

Cartwright, S. 1988. «Play Can Be the Building Blocks of Learning». Young Children, vol. $43, \mathrm{n}^{\circ} 5$ (juillet), p. 44-47.

Carugati, F., P. De Paolis, et G.A. Mugny. 1981. «Conflits des centrations et progrès cognitif III: régulations cognitives et relationnelles du conflit socio-cognitif». Bulletin de Psychologie, vol. 34, p. 845-852.

Carugati, F. et G.A. Mugny. 1985. «La théorie du conflit socio-cognitif». Psychologie sociale du développement cognitif, p. 57-70.

Clance, P.R., F. Dawson et S. Gill. 1975. Sex Differences in the Play Behavior of the Three Age Groups. Georgia State University, 17 p.

Connolly, J., A.B. Doyle et F. Ceschin. 1983. «Forms and Functions of Social Fantasy Play in Preschoolers». In Social and Cognitive Skills Sex Roles and Children's Play, sous la dir. de Marsha B. Liss. New York: Academic Press.

Cramer, P. et K.A. Hogan. 1975. «Sex Differences in Verbal and Play Fantasy». Development Psychology, vol. II, $\mathrm{n}^{\circ}$ 2, p. 145-154.

Cuffaro, H.K. 1984. «Dramatic Play. "The Experiences of Block Building"». In The Block Book, sous la dir. de E.S. Hirsch, p. 121-151. Washington: The National Association for the Education of Young Children.

Dalzon, C. 1988. «Conflit socio-cognitif et construction de la notion droite/gauche». In A.N. Perret-Clermont (Ed), Interagir et connaître: enjeux et régulations sociales dans le développement cognitif, p. 55-71. Cousset: Delval.

De Grandmont, N. 1989. Pédagogie du jeu. Jouer pour apprendre. Montréal: Éditions logiques, $213 \mathrm{p}$. 
De Ketele, J.M. et M. Postic. 1988. Observer les situations éducatives. Paris: P.U.F.

Dodge, D.T. 1979a. Blocks. A Creative Curriculum for Early Childhood Blocks. Washington: Creative Associates, Inc., 160 p.

Dodge, D.T. 1979b. Trainer's Guide to Blocks. A Creative Curriculum for Early Childhood. Washington: Creative Associates, Inc., 133 p.

Doise, W., G. Mugny et A.N. Perret-Clermont. 1974. «Ricerche preliminari sulla sociogenesi delle strutture cognitive». Lavore Educative, vol. 1, p. 33-50.

Doise, W., G. Mugny et A.N. Perret-Clermont. 1975. «Social Interaction and the Development Cognitive Operations». European Journal of Social Psychology, vol. 5 , p. $367-383$.

Doise, W. et G. Mugny. 1981. Le développement de l'intelligence. Paris: Interéditions.

Doise, W. 1985. «Le développement social de l'intelligence. Aperçu historique». In G. Mugny (Ed), Psychologie sociale du développement cognitif. Berne: Peter Lang, p. 39-55.

Doise, W. 1988. «Régulations sociales des opérations cognitives». In R.A. Hinde, A.-N. Perret-Clermont et J. Stevenson-Hinde, Relations interpersonnelles et développement des savoirs, p. 385-393. Cousset (Fribourg): Delval.

Doyle, A.B., J. Connoly et L.P. Rivest. 1980. «The Effect of Paymate Familiarity on the Social Interactions of Young Children». Child Development, vol. 51, p. 217223.

Doyle, A.-B. 1988. Différence dans le jeu symbolique social selon l'âge et la classe sociale: Relations avec la motivation et le développement cognitif. Rapport Final au Conseil québécois pour la recherche sociale, Projet RS 1004084 . Montréal: Université Concordia.

Doyle, A.-B., P. Doehring et S. De Lorimier. 1988. La communication au sein du jeu symbolique collectif: sa contribution au développement social et cognitif chez l'enfant de 3 à 9 ans. Colloque Jeu et Apprentissage tenu au Centre interdisciplinaire de recherches sur l'apprentissage et le développement en éducation.

Elder, J. et D. Pederson. 1978. «Preschool Children's Use of Objects in Symbolic Play». Child Development, vol. 49, p. 500-504.

Emler, N. et M. Glachan. 1985. «L'apprentissage social: perspectives récentes». In G. Mugny (Ed), Psychologie sociale du développement cognitif, p. 71-92. Berne: Peter Lang.

Erikson, E.H. 1940. «Further Explorations in Play Construction. Three Spacial Variables in their Relation to Sex ans Anxiety». Psychological Bulletin, 1941, vol. 38, p. 748. 
Erikson, E.H. 1940. «Studies in the Interpretation of Play». Genetic Psychological Monographs, vol. 22, p. 559-571.

Erikson, E.H. 1951. «Sex Differences in Play Configurations of Preadolescents». American Journal of Orthopsychiatry, vol. 21, $\mathrm{n}^{\circ} 4, \mathrm{p} .667-692$.

Erikson, E.H. 1982. Enfance et société. $7^{\mathrm{e}}$ éd. Neuchâtel: Delachaux et Niestlé.

Fagot, B. 1974. «Sex Differences in Toddlers' Behavior and Parental Reaction». Developmental Psychology, p. 554-558.

Farrell, M. 1957. «Sex Differences in Block Play in Early Childhood». Journal of Educational Research, vol. 51 (décembre) p. 281-284.

Farver, J.A.M. 1992. «Communicating Shared Meaning in Social Pretend Play». Early Childhood Research Quartely, vol. 7, p. 501-516.

Fein, G.G. 1975. «A Transformational Analysis of Pretending». Development Psychology, vol. 11, p. 291-296.

Fein, G.G. et A.R. Robertson. 1975. Cognitive and Social Dimensions of Pretending in Two-Year Olds. Détroit: Merril Palmer Institute.

Fein, G.G. 1979. «Play and the Acquisition of Symbols», In L, Katz (Ed), Current Topics in Early Childhood Education, p. 195-225. Norwood, N.J.: Ablex.

Fein, G.G. 1981. «Pretend Play: An Integrative Review». Child Development, vol. 52, p. 1095-1118.

Fein, G. et L. Stork. 1984. «Sociodramatic Play Class Effects in Integrated Preschool Class-room». Journal of Applied Developmental Psychology.

Foster, C. 1978. Blocks in the Early Childhood Program: Arizona. Arizona/Nevada: Child Development Association, 24 p.

Fraisse, J. 1985. Interactions sociales entre pairs et découverte d'une stratégie cognitive chez des enfants de 11 ans. Thèse de doctorat de troisième cycle. Aix-en-Provence: Université de Provence.

Fraisse, J. 1987. «Étude du rôle perturbateur du partenaire dans la découverte d'une stratégie cognitive chez des enfants de 11 ans en situation d'interaction sociale». Bulletin de psychologie, vol. 40, p. 943-952.

Freud, S. 1938. Three Contributions to the Theory of Sex. The Basic Writing of Sigmund Freud. New York: Modern Library.

Freud, S. 1962, 1982. Trois essais sur la théorie de la sexualité. Paris: Gallimard.

Garnier, C. et J.C. Brief. 1985. "Analysis of Cooperation within Groups of Preschool Children». Cahier de psychologie cognitive (I.S.S.B.D.), International Society for the Study of Behavioral Development, Eight Biennal Meeting, vol. 5, nos 3-4. 
Garnier, C. 1987. Représentation des composantes de la coopération au préscolaire dans les situations ludiques libres. Séminaire sur la représentation, $\mathrm{n}^{\circ} 23$. Université $\mathrm{du}$ Québec à Montréal: CIRADE.

Garnier, C., A. Latour, J. Ferraris et M. Quesnel. 1991. «Coopération in Groups of Preschool Children during Free Play». Rôle des activités ludiques dans le développement des composantes comportementales et représentationnelles de la coopération. Rapport de recherche du Fonds des chercheurs et aide à la recherche (FCAR), FCAR 89 EQ3058, 122 p.

Garvey, C. 1974. «Some Proprieties of Social Play». Merrill-Palmer Quaterly, vol. 20, p. $163-180$.

Garvey, C. 1977. Play. Cambridge: Harvard University Press.

Garvey, C. et R. Berndt. 1977. Organization of Pretend Play. Paper presented at the meeting of the American Psychology Association, Chicago.

Garvey, C. 1982. «Communication and the Development of Social Role Play». In D. Foerbes et M. Greenberg (Eds), New Directions in Child Development: the Development of Playful Behavior in Children. San Francisco: Jossey-Bass.

Gianini, B.E. 1974. Du côté des petites filles. Paris: Éditions des Femmes, 257 p.

Giffin, H. 1984. "The Coordination of Meaning in the Creation of Sharer Make-believe Reality». In Symbolic Play: The Development of Social Understanding, sous la dir. I. Bretherton. Montréal: Academic Press.

Gilly, M., J. Fraisse et J.P. Roux. 1984. «Efficacité comparée du travail individuel et du travail en interaction socio-cognitive dans l'appropriation et la mise en oeuvre de règles de résolution chez des enfants de 11-12 ans». Cahier de psychologie cognitive, vol. 4, p. 171-188.

Gilly, M., A. Blaye et J.P. Roux. 1988. «Élaboration de constructions cognitives individuelles en situations socio-cognitives de résolution de problèmes». In G. Mugny et J.A. Perrez (Eds), Psicologia social del desarollo cognitivo. Barcelone: Anthropos.

Gilly, M. 1988. «Interactions entre pairs et constructions cognitives: modèles explicatifs». In A.N. Perret-Clermont (Ed), Interagir et connaître: enjeux et régulations sociales dans le développement cognitif, p. 19-28. Cousset (Fribourg): Delval.

Gilly, M. et J.P. Roux. 1988. «Social Marking in Ordering Tasks: Effects and Action Mechanisms». European Journal of Social Psychology, vol. 18, p. 251-266.

Gilly, M. 1989. «À propos de la théorie du conflit et des mécanismes psycho-sociaux des constructions cognitives: perspectives actuelles et modèle explicatif. Construction des savoirs». Obstacles et conflits, sous la direction de N. Bednarz et C. Garnier. Colloque international: Obstacle épistémologique et conflit socio-cognitif. CIRADE, Montréal: Édition Agence D’Arc, p. 162-182. 
Göncü, A. 1985. Toward an Interactional Model of Developmental Changes in Social Pretend Play. ERIC Clearing-house on Elementary and Early Childhood Education (n ${ }^{\circ}$ 246032). Urbana, Il.

Göncü, A. 1987a. «The Role of Adults and Peers in the Socialization of Play during Preschool Years». In G. Gasto, S. Ascione et M. Saleri (Eds), Current Perspectives in Infancy and Early Childhood Research, p. 33-41. Logan, U.T.: Early Intervention Research Institute Press.

Göncü, A. 1987b. «Toward and Interactional Model of Developmental Changes in social Pretend Play». In L. Katz (Ed), Current Topics in Early Childhood Education, vol. 7, p. 108-125. Norwood, N.J.: Ablex.

Göncü, A. et F. Kessel. 1988. «Preschoolers' Collaborative Construction in Planning and Maintaining Imaginative Play». International Journal of Behavioral Development, vol. 11, n 3, p. 327-344.

Goodfader, R.A. 1982. «Sex Differences in the Play Constructions of Pre-school Children». Smith College Studies in Social Work, vol. 52, n 2, p. 129-144.

Gowen, J. 1978. Structural Elements of Symbolic Play of Preschool Children. Paper presented at the meeting of the American Psychological Association (august). Toronto.

Goyette, Gabriel. 1988. La méthode de l'observation et le développement des sciences de l'éducation. Montréal: Université du Québec à Montréal, Département des sciences de l'éducation (texte inédit).

Hartley, R.E., L.K. Frank et R.M. Goldenson. 1952. Understanding Children's Play. New York.: Columbia University Press.

Hennebelle, N. 1986. Rôle de l'interaction sociale dans une tâche d'extraction de critères. Mémoire de D.E.A. inédit. Aix-en-Provence: Université de Provence.

Hinde, R.A., A.-N. Perret-Clermont et J. Stevenson-Hinde. 1988. Relations interpersonnelles et développement des savoirs. Fondation Delval, 529 p. (385-393).

Howes, C. 1988. «Peer Interaction in Young Children». Monographs of the Society for Research in Child Development, vol. 53, $\mathrm{n}^{\circ} 1$ (séries $n^{\circ} 217$ ).

Howes, C. 1993. «Commentary». Human Development, vol. 36, p. 241-246.

Howes, C., K. Droege et C.C. Matheson. 1994. «Play and Communicative Processes within Long-and-Short-Term Friendship Dyads». Journal of Social and Personal Relationships (Sage, London, Thousand Oaks, CA and New Delhi), vol. 11, p. 401-410.

Huberman, M.A. et M.B. Miles. 1991. Analyse des données qualitatives. Recueil de nouvelles méthodes. Bruxelles: De Boeck-Wesmael, s.a. 480 p. 
Hulson et Reich. 1931. «Blocks and the Four Year Old». Childhood Education, vol. 8, p. 66-68.

Inhelder, B., H. Sinclair et M. Bovet. 1974. Apprentissage et structures de la connaissance. Paris: P.U.F.

Iwanaga, M. 1973. «Development of Interpersonal Play Structures in 3, 4 and 5 Year Old Children». Journal of Research and Development in Education, vol. 6, p. 7182.

Jackowitz, E.R. et M.W. Watson. 1980. «The Development of Object Transformations in Early Pretend Play». Developmental Psychology, vol. 16, p. 543-549.

Jeffree, D. et R. McConkey. 1976. «An Observation Scheme for Recording Children's Imaginative Doll Play». Journal of Child Psychology and Psychiatry, vol. 17, p. 189-197.

Johnson, H.M. 1974. The art of Block Building. In The Block Book, sous la direction de E.S. Hirsch. Washington: The National Association for the Education of Young Children.

Johnson, J.E., J. Ershler et C. Bell. 1980. «Play Behavior in a Discovery-based and a Formal Education Preschool Program». Child Development, vol. 51, p. 271-274.

Johnson, J.E. et J. Ershler. 1981. «Developmental Trends in Preschool Play as Function of Classroom Setting and Child Gender». Child Development, vol. 52, p. 995-1004.

Kane, S.R. et H.G. Furth. 1993. «Children Constructing Social Reality: a Frame Analysis of Social Pretend Play». Human Development, vol. 36, p. 199-214.

Kinsman, C. et L. Berk. 1979. «Joining the Bloc and House Keeping Areas». Young Children, vol. 35 , p. 66-75.

Kohlberg, L. 1966. «A Cognitive Developmental Analysis of Children's Sex-role Concepts and Attitudes». In The Development of Sex Differences, sous la direction de E.E. Maccoby-Standford, p. 82-173. California: Standford University Press.

L'Écuyer, R. 1990. Méthodologie de l'analyse développementale de contenu. Méthode GPS et concept de soi. Québec: P.U.Q.

Lafortune, M. 1989. Le psychologue pétrifié ou Du modèle expérimental comme perversion du discours humain. Montréal: Louise Courteau, Éditrice, $176 \mathrm{p}$.

Leeb-Lundberg, K. 1970. «Kindergarten Mathematics Laboratory». The Arithmetic Teacher, vol. $17, \mathrm{n}^{\circ}$ 5, p. 372-386.

Leeb-Lundberg, K. 1984. «The Block Builder Mathematician». In The Block Book, sous la dir. de E.S. Hirsch, p. 63-101. Washington: The National Association for the Education of Young Children. 
Liedtke, W. 1975. «Experiences with Blocks in Kindergarten». The Arithmetic Teacher, p. $406-412$.

Marc, E. et D. Picard. 1989. L'interaction sociale. Paris: P.U.F.

Margolin, E.B. et D.A. Leton. 1961. «Interest of Children in Block Play». Journal of Educational Research, vol. 55, p. 13-18.

Massey, M. 1969. Kindergarten Children's Behavior in Block Building Situation. Unpublished Doctoral dissertation. Florida: Florida State University.

Matthews, W.S. 1977. «Modes of Transformation in Initiation of Fantasy Play». Developmental Psychology, vol. 13, p. 212-216.

McLyod, V. C. 1980. «Verbally Expressed Modes of Transformation in the Fantasy Play of Black Preschool Children». Child Development, vol. 51, p. 1133-1139.

Mead, M. 1966. L'un et l'autre sexe. Paris: Denoël.

Milar, S. 1968. La psychologie du jeu chez les enfants et les animaux. Paris: Petite Bibliothèque Payot. $309 \mathrm{p}$.

Ministère de l'Éducation du Québec. 1982. Guide pédagogique, préscolaire. Guide général d'interprétation et d'instrumentation pédagogique pour le programme d'éducation préscolaire. Québec: Direction générale des programmes du primaire (Q.G.P.P.).

Ministère de l'Éducation du Québec. 1981. Programme d'éducation préscolaire. Québec: direction générale du primaire (D.G.P.P.)

Mischel, W. 1970. «Sex Typing and Socialization». In Carmichael's Manuel of Child Psychology, sous la dir. de P.H. Mussen, vol 11, p. 3-72. New York: Wiley.

Moffitt, M.W. 1984. «Children Learn about Science through Block Building». In The Block Book, sous la dir. de E.S. Hirsch, p. 51-101. U.S.: National Association for the Education of Young Children.

Moscovici, S. (éd). 1972. Introduction à la psychologie sociale. Paris: Larousse, vol. 1 et 2 .

Moscovici, S. 1976. Social Influence and Social Change. London: Academic Press.

Moscovici, S. 1984. Psychologie sociale. Paris: P.U.F.

Moyer, K. et V.H. Gilner. 1956. «Experimental Study of Children's Preferences and Use of Block Play». Journal of Genetic Psychology, vol, 89, p. 3-10.

Mueller, E. et T.A. Lucas. 1975. «A Developmental Analysis of Peer Interaction among Toddlers». In Peer Relations and Friendship, sous la dir. de M. Lewis et L. Rosenblum. New York: Wiley. 
Mugny, G., W. Doise et A.N. Perret-Clermont. 1976. «Conflit, décentrations et progrès cognitif». Bulletin de psychologie, vol. 29, p. 199-204.

Mugny, G. 1985. Psychologie sociale du développement cognitif. New York: Peter Lang, $283 \mathrm{p}$.

Mussati, T. 1983. «Échanges dans une situation de jeux de "faire-semblant"». In M. Stamback et al., Les bébés entre eux. Paris: P.U.F.

Nadel, J. 1986. Imitation et communication entre jeunes enfants. Paris: P.U.F.

Overton, W.F. et J.P. Jackson. 1973. «The Representation of Imagined Objects in Action Sequences: a Developmental Study». Child Development, vol. 44, p. 309314.

Papalia, D.E. et S.W. Olds. 1989. Le développement de la personne. Montréal: Les Éditions HRW Ltée., 505 p.

Parten, M.B. 1933. «Social Participation among Preschool Children». Journal of Abnormal and Social Psychology, vol. 27, p. 243-269.

Perret-Clermont, A.N. 1976. L'interaction sociale comme facteur de développement cognitif. Thèse de doctorat. Genève: Université de Genève.

Perret-Clermont, A.N. 1979. La construction de l'intelligence dans l'interaction sociale. Berne: Peter Lang.

Perret-Clermont, A.N. 1986. «Interactions sociales et processus de connaissances». Cahiers de psychologie de l'Université de Neuchâtel, $\mathrm{n}^{\circ}$ 24, p. 5-13.

Perret-Clermont, A.N. 1988. Interagir et connaître: enjeux et régulations sociales dans le développement cognitif. Cousset (Fribourg): Delval.

Piaget, J. 1932. Le jugement moral chez l'enfant. Neuchâtel: Delachaux et Niestlé.

Piaget, J. 1962. Play, Dreams, and Imitation in Childhood. New York: Norton.

Piaget, J. 1964, 1978. La formation du symbole chez l'enfant. Paris: Delachaux et Niestlé Spes., 310 p.

Piaget, J. 1964. Six études de psychologie. Genève: Gonthier.

Piaget, J. 1965. The Child's Conception of Number. New York: Norton.

Piaget, J. et B. Inhelder. 1966. L'image mentale chez l'enfant. Paris: P.U.F.

Piaget, J. 1967. La psychologie de l'intelligence. Paris: Armand.

Piaget, J. 1970. Psychologie et épistémologie. Paris: Denoël-Gonthier.

Piaget, J. 1975. L'équilibration des structures cognitives. Paris: P.U.F. 
Pratt, C. 1933/1966/1984. The Art of Building. New York: Bank Street College of Education Publications.

Provenzo, E.F. et A. Brett. 1983. The Complete Bock Book. New York.: Syracuse University Press.

Reifel, S. et P.M. Greenfield. 1982. "Structural Development in a Symbolic Medium: the Representational Use of Block Construction». In Action and Thought: from Sensorimotor Schemes to Symbolic Operations, sous la dir. de G.E. Torman. New York: Academic Press.

Reifel, S. 1984. «Block Construction: Children's Developmental Landmarksin Representation of Space», Young Children (novembre), p. 61-67.

Robinson, E. 1958. The Form and Imaginative Content of Children's Block Building. Unpublished Doctoral dissertation. Minnesota: University of Minnesota.

Robinson, H. 1977. Exploring Teaching Early Childhood Education. Boston: Allyrand Bacon, inc.

Robinson, E, R.K. Silbereisen et A. Claar. 1985. «Le développement de la communication». In G. Mugny (Ed), Psychologie sociale du développement cognitif, p. 109124. Berne: Peter Lang.

Rogers, D. 1985. «Relationships between Block Play and the Social Development of Young Children». Early Child Development and Care, vol. 20, p. 245-261.

Ross, H.S., B.D. Goldman et D.F. Hay. 1979. «Approach and Exploration of a Novel Alternative by 12 Month Old Infants». Journal of Experimental Child Psychology, vol. 13 , p. $85-93$.

Roux, J.P. et M. Gilly. 1984. «Aide apportée par le marquage social dans une procédure de résolution chez des enfants de 12-13 ans: données et réflexions sur les mécanismes». Bulletin de psychologie, vol. 38, p. 145-155.

Roux, J.P. et M. Gilly. 1988. «Contribution à l'étude des mécanismes d'action du marquage social dans une tâche d'ordination à 12-13 ans». In A.N. PerretClermont (Ed), Interagir et connaître: enjeux et régulations sociales dans le développement cognitif, p. 153-165. Cousset: Delval.

Rubin, K.H. 1977. «Play Behaviors of Young Children», Young Children, vol. 32, p. 16-24.

Rubin, K.H. 1980. «Fantasy Play: Its Role in the Development of Social Skills and Social Cognition». In Children's Play, sous la dir. de K.H. Rubin, New Directions for Child Development, $n^{\circ}$ 9. San Francisco: Jossey Bass.

Rubin, K.H., T.L. Maioni et M. Hornung. 1976. «Free Play Behaviors in Middle - and Lower - Class Preschoolers: Parten and Piaget Revisited». Child Development, vol. 47, p. 414-419. 
Rubin, K.H., G. Fein et B. Vanderberg. 1984. «Play in Socialization, Personality and Social Development». Handbook of Child Psychology, sous la direction de E.M. Hetherington (Ed), vol, 1V. New York: Wiley.

Rudolf, P.H. et M. Cohen. 1964. The Many Purposes of Block Building and Woodwork in Kindergarden: a Year of Learning. New York: Appelton, Century Crafts.

Saltz, E. et J. Johnson. 1974. «Training for Thematic-fantasy Behavior in Preschool Children: Relations among Gender, Age, Season and Location». Child Development, vol. 47, p. 1182-1185.

Saltz, E., D. Dixon et J. Johnson. 1977. «Training Disadvantages Preschoolers on Various Fantasy Activities: Effects on Cognitive Functioning and Impulse Control». Child Development, vol. 48, p. 367-380.

Saltz, E. et J. Brodie. 1982. «Pretend-play Training in Childhood: a Review and Critique». In The Play of Children: Current Theory and Research, sous la dir. de D.J. Pepler et K.H. Rubin. Basel Switzerland: Karger Ag.

Santa, M.A. 1980. Units Blocks. A Curriculum for Early Learning. US: District of Colombia (août), 23 p.

Schirrmacher, R. 1975. Effects of Adult Modeling on the Developmental Level of Children's Block Constructions Measured on an Ordinal Scale. Unpublished doctoral dissertation. Illinois: University of Illinois.

Schuster, J. 1973. Sex Differences and Within Sex Variations in Children's Block Construction. Unpublished doctoral dissertation. New York.: New York University.

Schwartzman, H.B. 1976. «Children's Play: a Sideways Glance at Make-believe». In The Anthropological Study of Play: Problems and Prospects, sous la dir. de D.F. Lancy et B.A. Tindall. Cornwall, N.Y: Leisure Press.

Schwartzman, H.B. 1978. Transformation: The Anthropology of Children's Play. New York: Plentum.

Sears, R.R. 1947. «Influence of Methodological Factors of Doll-play Performance». Child Development, vol. 18, p. 190-197.

Sinclair, H., M. Stamback, I. Lézine, S. Rayna et M. Verba. 1982. Les bébés et les choses. Paris: Presses universitaires de France, 200 p.

Singer, D.G. 1973. «Ideational Creativity and Behavioral Style in Kindergarden Aged Children». Development Psychology, vol. 8, p. 154-161.

Smedslund, J. 1966. «Les origines sociales de la décentration». In P. Bresson, H. De Montmollin (Eds), Psychologie et épistémologie génétiques, thèmes piagétiens, p. 165-167. Paris: Dunod. 
Smith, P. 1977. «Behaviorally Based Masculine and Feminine Activity Preference Scales for Preschoolers: Correlates with other Classroom Behaviors and Cognitive Tests». Child Development, vol. 48, p. 389-404.

Smith, P.K. et K. Connoly. 1972. «Patterns of Play and Social Interaction in Preschool Children». In Ethological Studies of Child Behavior, sous la dir. de N. Blurton Jones. Cambridge: Cambridge University Press.

Smylansky, S. 1968. The Effects of Sociodramatic Play on Disadvantaged Preschool Children. Toronto: John Wiley and Sons.

Sprung, B. 1975. Non-sexist Education for Young Children. New York: Citation Press.

Sprung, B. 1978. Perspectives on Non-sexist in Early Childhood Education. New York: Teachers College Press.

Stamback, M., M. Barrière, I. Bonica, R. Maisonnet, T. Musatti, S. Rayna et M. Verba. 1983. Les bébés entre eux. Paris: Presses universitaires de France, $191 \mathrm{p}$.

Stamback, M. et H. Sinclair. 1990. «Introduction». Les jeux de fiction entre enfants de 3 ans, sous la dir. de M. Stamback et H. Sinclair. Collection Psychologie d'aujourd'hui. Paris: P.U.F.

Strayer, F. et R. Gauthier. 1982 . «L'approche éthologique de l'observation du comportement». Apprentissage et socialisation, vol. 5, n ${ }^{\circ} 1$, p. 12-23.

Sutton-Smith, B. 1971. «Boundaries». In Child's Play, sous la dir. de R.E. Herron et B. Sutton-Smith . New York: Wiley.

Sutton-Smith, B. 1979. Play and Learning. New York: Gardner Press.

Thériault, J., D. Doyon, M. Doucet et S. Van Tham. 1987. Le potentiel et la pertinence du matériel éducatif des classes maternelles. Chicoutimi: Université du Québec à Chicoutimi, Document \#1.

Toshiko, U., S. Tomoko et M. Takahashi. 1974. «Interaction Process of Two Children». The Japanese Journal of Educational Psychology, vol. 22, p. 176-180.

Trewarthen, C. 1977. «Descriptive Analysis of Infant Communicative Behavior». In H.R. Schaffer (Ed), Studies in Mother-Infant Interactions. London: Academic Press.

Van Alystyne, D. 1932. Play Behavior and Choice of Play Materials of Preschool Children. Chicago: The University of Chicago Press.

Vandell, D.L. et E.C. Mueller. 1980. «Peer Play and Friendships during the First Two Years». In Friendship and Social Relations in Children, sous la dir. de H.C. Foot., A.J. Chapman et J.R. Smith. London: Wiley.

Vandenplas-Holper, C. 1987. Education et développement social de l'enfant. Paris: Presses universitaires de France. 
Varma, M. 1980. «Sex Stereotyping and Block Play of Preschool Children», Indian Educational Review, p. 32-37.

Verba, M. 1985. Construction interindividuelle des jeux de fiction chez les jeunes enfants. Communication aux $3 \mathrm{e}$ journées du Groupe francophone d'étude du développement psychologique de l'enfant jeune. Nice, 10-11 mai.

Verba, M. 1987. Cooperation, Tutoring and Imitation in Social Symbolic Play: the Role of the Older Child. VIII congrès biennal de la S.R.C.D., 23-26 avril, Baltimore.

Verba, M. 1990. «Construction et partage de signification dans les jeux de fiction entre enfants». In Les jeux de fiction entre enfants de 3 ans, sous la dir. de M. Stamback et H. Sinclair, p. 7-23. Paris: Presses universitaires de France.

Vlietstra, A. 1978. «Exploration and Play in Preschool and Adults». Child Development, vol. 49 , p. $235-238$.

Vygotsky, L.S. 1966. «Play and its Role in the Mental Development of the Child». Soviet Psychology, vol. 12, p. 62-76.

Vygotsky, L.S. 1967. «Play and its Role in the Mental Development of the Child». Soviet Psychology, vol. 5, p. 6-18.

Vygotsky, L.S. 1978. Mind in Society: The Development of Higher Mental Processes. Cambridge, Mass: Harvard University Press.

Wallon; H. 1949. Les origines du caractère chez l'enfant. Paris: P.U.F.

Wambach, R.L. 1974. Sex Difference in the Play Configurations of Pre-adolescent: a comparative Study. (Doctoral Dissertation. University of Washington). Ann Arbor, Michigan: University Microfilms.

Watson, M.W. et K.W. Fischer. 1977. «A developmental Sequence of Agent Use in Late Infancy». Child Development, 48, p. 828-836.

Watson, M.W. et K.W. Fischer. 1980. «Development of Social Roles in Elicid and Spontaneous Behavior during the Preschool Years». Developmental Psychology, 16.

Wertsch, J.V. 1978. «Adult-Child Interaction and the Roots of Metacognition».Quartely Newsletter of the Institute for Comparative Human Development, vol. 2, p. 15-18.

Wertsch, J.V. 1979. «From Social Interaction to Higher Psychological Processes. A Clarification and Application of Vygotsky's Theory». Human Development, vol. 22 , p. 1-22.

Wertsch, J.V. 1980. «The signifiance of dialogue in Vygotsky's account of social, egocentric and inner speech». Contempory Educational Psychology, 5, p. 150162. 
Wertsch, J.V. et C.A. Stone. 1985. «The Concept of Internalization in Vygotsky's Account of the Genesis of Higher Mental Functions». In J. Wertsch (Ed), Culture, communication and cognition: Vygotskian perspectives, p. 162-179. Cambridge: Cambridge University Press.

Wilcox, A. 1979. Sex Differences in the Play Configuration of Pre-adolescents: $a$ Replication and Revision. New Orleans: Meeting of the Southeaster Psychological Association, $18 \mathrm{p}$.

Winnykamen, F. 1985. «Imitation et acquisition par observation: études récentes et perspectives». In J. Bidaud et M. Richelle (Eds), Psychologie développementale: problèmes et réalités. Bruxelles: Pierre Mardaga.

Winnykamen, F. 1987. Imitation - modélisation: modalités sociales des interactions. Thèse de doctorat d'État. Paris: Université de Paris V.

Winnykamen, F. 1988. «Quelques réflexions sur la fonction acquisitive de l'imitation chez l'enfant». Psychologie française, vol. 33.

Wolf, D.P., J. Rygh et J. Altshuler. 1984. «Agency and Experience: Action and States in Play Narratives». In Il Breterton (Ed), Symbolic Play, the Development of social Understanding, p. 195-217. Orlando: Academic Press.

Wolfgang, C.H., B. Mackender et M.E. Wolfgang. 1981. Growing and Learning Through Play. Activities for Preschool and Kindergarten Children. A Parent/Caregiver Book. Irvine, Californie: Instruction/McGraw-Hill.

Zhou, R.M. 1987. Marquage social, conduites de partage et construction de la notion de conservation chez des enfants de 5-6 ans. Thèse de doctorat. Aix-en-Provence: Université de Provence.

Zhou, R.M. 1988. «Norme égalitaire, conduites sociales et partage et acquisition de la conservation des quantités». In A.N. Perret-Clermont (Ed), Interagir et connaître: enjeux et régulations sociales dans le développement cognitif, p. 167-180. Cousset, Delval. 